

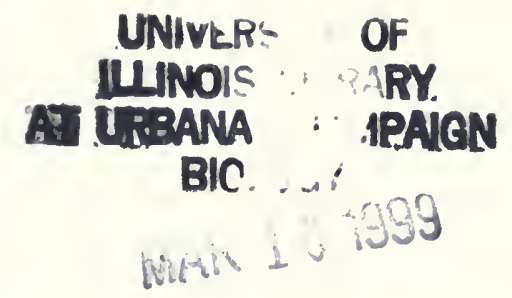


map for no .85 inside back over 


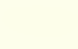


590.5

FI

N.S.

10. 86

$109-$ thro:

Coology

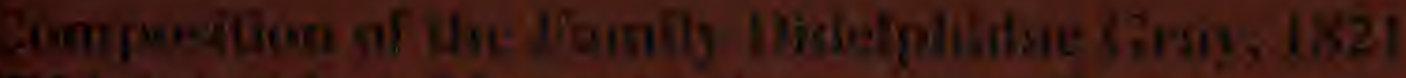

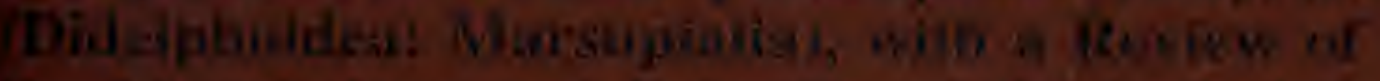

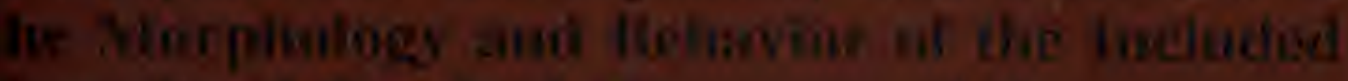

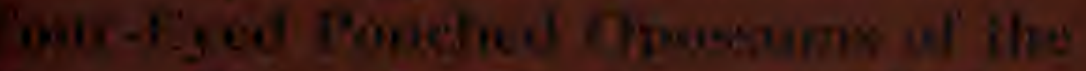

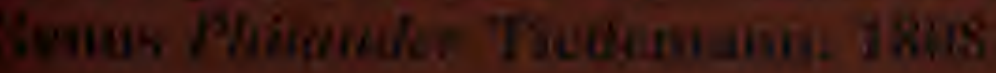

withe for wimetio 


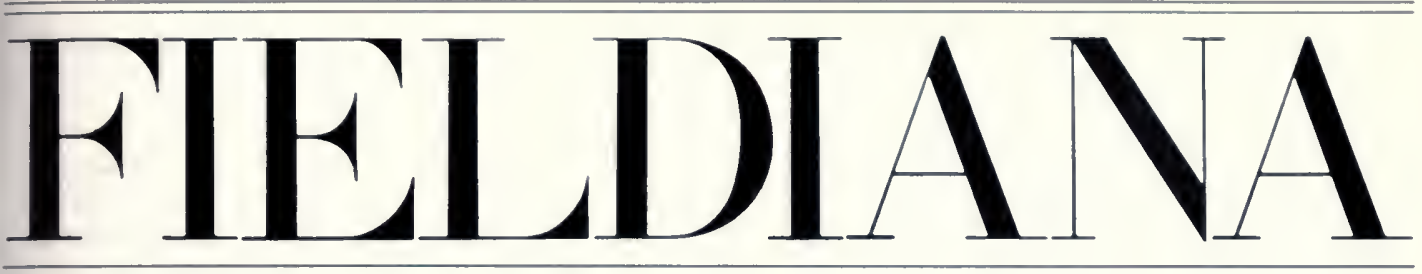

\section{Zoology}

NEW SERIES, NO. 86

Composition of the Family Didelphidae Gray, 1821 (Didelphoidea: Marsupialia), with a Review of the Morphology and Behavior of the Included Four-Eyed Pouched Opossums of the Genus Philander Tiedemann, 1808

Philip Hershkovitz $\dagger$

\section{Curator Emeriu.}

Division of Mammals

Department of Zoology

Field Museum of Natural History

Roosevelt Road at Lake Shore Drive

Chicago, Illinois 60605-2496 USA

Accepted April 4, 1996

Published May 30, 1997

Publication 1485
BIOLOGY LIBRARY

101 BURRILL HALL

OCT 161997 
(C) 1997 Field Museum of Natural History

ISSN 0015-0754

PRINTED IN THE UNITED STATES OF AMERICA 


\section{Table of Contents}

ABSTRACT ……...................................

INTRODUCTION ................................ 1

AbBreviations ...................................... 2

Didelphidae: Taxonomic Status, The

GENERA ......................................... 2

A MorPhological TEST ........................ 4

Genus $P_{\text {hilander Tiedemann (Four-Eyed }}$

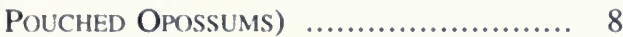

Synonymy ….................................. 8

Distribution ...................................... 8

Nomenclature .................................. 10

External Characters and Comparisons ...... 11

Cranial Characters and Comparisons ........ 15

Dental Characters and Comparisons ......... 17

Descriptions of Individual Teeth of Philander .......................................... 18

Dental Abnormalities .......................... 23

Sexual Dimorphism .......................... 24

Karyology ......................................... 24

Parasites ............................................ 25

Species and Named and UnNamed Sub-

SPECIES OF PHILANDER OPPOSUM ............. 32

Philander opossum Linnaeus ................ 33

Philander opossum opossum Linnaeus ..... 35

Philander opossum quica Temminck ........ 40

Philander opossum frenatus Olfers ......... 51

Philander opossum melanurus Thomas .... 51

Philander opossum subsp. nov. 1 ........... 52

Philander opossum subsp. nov.? ........... 52

Philander opossum fuscogriseus J. A. Allen 52

Philander andersoni Osgood ................ 59

Philander andersoni andersoni Osgood .... 61

Philander andersoni mcilhennyi Gardner and Patton

Behavior of the Four-Eyed Pouched

OpOSSUMS (PHILANDER OPOSSUM)

AlpHaBETical List of COLleEting LoCALITIES

GAZETTEER .......................................... 85

ACKNOWLEDGMENTS .............................. 95

LitERATURE CITED .............................. 95

\section{List of Illustrations}

1. The four genera of largest American marsupials

2. Skulls of the four largest American marsupials
3. Hindfoot of Chironectes minimus

4. Dorsal pigmentation of Philander opossum and $P$. andersoni ................ 9

5. Geographic distribution of Philander .... 10

6. Cheiridia of Philander opossum .......... 16

7. Caudal segments of the four largest American opossums

8. Scales and bristles of Philander opossum tail

9. Transitional caudal vertebrae of Philander opossum ................................... 20

10. Points of cranial measurements ........... 22

11. Diagram of didelphid skull ................. 23

12. Left side of Philander opossum skull and mandible ................................. 25

13. Philander opossum skull ................... 26

14. Tooth rows of Philander opossum ....... 27

15. Upper incisors and canine of Philander opossum, Caluromys philander, Lutreolina crassicaudata, Metachirus nudicaudatus ........................................ 28

16. Staggered $\mathrm{i}_{3}$ in Philander opossum ....... 29

17. Deciduous premolar 3 and molars ........ 30

18. Accessory molar cusps .................... 31

19. Conules of $\mathrm{m}^{3}$ of Philander from various geographic localities ................... 32

20. $\mathrm{M}^{3}$ of Philander opossum ................. 33

21. Lectotypes of Philander opossum ........ 39

22. South American collecting localities ..... 81

\section{List of Tables}

1. Summarized measurements of Philander opossum and $P$. andersoni subspecies

2. Dimensions and proportions of limb bones ........................................ 15

3. Vertebral bone formulae .................. 19

4. Nipple formulae ........................ 21

5. Symbols for cranial bones and foramina ................................. 24

6. Symbols for dental elements .............. 34

7. Conules of $\mathrm{m}^{3}$............................ 35

8. Karyotypes of largest American marsupials ..................................... 35

9. Measurements of Philander opossum opossum .................................. 36

10. Measurements of Philander opossum quica

11. Measurements of Philander opossum melanurus 
12. Measurements of Philander opossum fuscogriseus

13. Ratios of hairy tail bases to tail lengths

14. Sympatry between Philander species

15. Measurements of Philander andersoni andersoni 59

60 62
16. Measurements of Philander andersoni mcilhennyi

17. Reproductive condition of 22 female Philander opossum from Nicaragua ..... 68

18. Body mass and above-ground foraging .. 72

19. Duration of residence in study area 78 


\title{
Composition of the Family Didelphidae Gray, 1821 (Didelphoidea: Marsupialia), with a Review of the Morphology and Behavior of the Included Four-Eyed Pouched Opossums of the Genus Philander Tiedemann, 1808
}

\author{
Philip Hershkovitz $\dagger$
}

\begin{abstract}
The generic content of the family Didelphidae as currently conceived consists of Didelphis, Philander, Chironectes, and Lutreolina. It is shown that most defining characters of the Didelphidae determined from those of the genus Didelphis are highly derived and not shared by Chironectes or Lutreolina. Accordingly, the family content was restricted to Didelphis and the genus Philander, which is smaller and less derived than Didelphis. The genus Didelphis had previously undergone a partial taxonomic revision. A complete revision of the genus Philander now follows. Two species of the genus have been recognized. Comparisons were made with outgroups Chironectes and Lutreolina. Habits of Philander, insofar as known, are described. The more primitive genus Metachirus, which is sometimes regarded as a didelphid, is being treated separately.
\end{abstract}

\section{Introduction}

The four-eyed pouched opossums, genus Philander Tiedemann, 1808, most nearly related to the larger opossums of the genus Didelphis Linnaeus, 1758, are common throughout much of the neotropics from northern Argentina into Tamaulipas, México. About 20 Linnaean names have been proposed for four-eyed pouched opossums, but only two species are recognized here. The earliest known, $P$. opossum Linnaeus, 1758, with its geographic distribution nearly equal to that of the genus, is the smaller of the two and generally less darkly colored. Philander andersoni Osgood, mainly western Amazonian in distribution, contains two subspecies. One, $P$. andersoni andersoni Osgood, occurs in Perú and in the Territorio Federal Amazonas, Venezuela. The wide gap in distribution between the two countries may be for lack of collecting or the result of a climatic event. The blackish $P$. andersoni mcilhennyi Gardner and Patton is known only from the upper Río Purús basin in Amazonian Perú.
This report is the author's sixth on New World marsupial systematics and behavior. The series began in 1992 with a taxonomic revision of the gracile mouse opossum, genus Gracilinanus (Marmosidae) (Hershkovitz, 1992a). It was followed in the same year by a critical examination of the significance of ankle bones as phylogenetic indicators (Hershkovitz, 1992b). The description of a seemingly abnormal staggered third lower incisor, which proved to be the hallmark of all didelphimorphs since the earliest Cretaceous, if not late Jurassic, was published in 1995. In press is a taxonomic review and life history account of the relict Chilean mouse opossum Dromiciops (Microbiotheriidae). Also awaiting publication is another review, that of the brown four-eyed pouchless opossum, genus Metachirus. This taxon was used at first as an outgroup for the Philander opus. As work progressed, however, Metachirus took on greater importance and is being completed under its own title.

In preparation is a monograph of living New 
World marsupials from which the above-mentioned articles have been partially derived and elaborated.

\section{Abbreviations}

The following abbreviations are used for the institutions where the specimens examined are preserved.

$$
\begin{aligned}
\text { AMNH }= & \text { American Museum of Natural His- } \\
& \text { tory, New York } \\
\mathrm{BM}(\mathrm{NH})= & \text { British Museum (Natural History), } \\
& \text { London } \\
\text { FMNH }= & \text { Field Museum of Natural History, } \\
& \text { Chicago } \\
\text { MVZUC }= & \text { Museum of Vertebrate Zoology, Uni- } \\
& \text { versity of California, Berkeley } \\
\text { USNM = } & \text { National Museum of Natural History, } \\
& \text { Washington, D.C. } \\
\text { USPMZ }= & \text { Universidade de São Paulo Museu de } \\
& \text { Zoologia, São Paulo, Brasil } \\
\text { MPEG = } & \text { Museu Paraense Emilio Goeldi, Be- } \\
& \text { lém, Brazil } \\
\text { UKMNH = } & \text { University of Kansas Museum of } \\
& \text { Natural History, Lawrence } \\
\text { LSUMZ = } & \text { Louisiana State University Museum } \\
& \text { of Zoology, Baton Rouge } \\
\text { MNRJ = } & \text { Museu Nacional, Rio de Janeiro } \\
\text { RMNH }= & \text { Rijksmuseum van Naturalijke Histo- } \\
& \text { rie, Leiden }
\end{aligned}
$$

\section{Didelphidae: Taxonomic Status, The Genera}

The family Didelphidae, erected by Gray, 1821, included all then-known American marsupials divided into the genera Didelphis Linnaeus and Cheironectes [sic] Illiger. It was not until late in the 20th century that critical attention was paid to higher systematic categories of living New World marsupials.

The early arrangement of the taxa persisted, nevertheless, as if all female marsupials were pouched and all individuals prehensile-tailed, scansorial, and members of the genus Didelphis. Discovery of the first caenolestid by Tomes in 1863 added another dimension to the concept of American marsupials by directing attention to the existence of nonpouched, non-prehensile-tailed and terrestrial American marsupials. Notwithstanding, apart from accommodations made in catalogs for the increasing number of newly discovered taxa, no significant changes were made in the classifications. Recent catalogers (Cabrera, 1958; Hall \& Kelson, 1959; Honecki et al., 1982; Gardner, 1993) recognized the three current orders of American marsupials, each with a single family, its content as found in the literature. The categories, with the numbers of their respective living genera and species in parentheses (ex Gardner, 1993), follow:

Order Didelphimorphia

Family Didelphidae (15 genera, 63 species)

Order Paucituberculata

Family Caenolestidae $(3,5)$

Order Microbiotheria

Family Microbiotheriidae $(1,1)$

Research by Reig et al. (1977) on chromosomes of the Didelphidae resulted in significant advances. Three karyotypes were distinguished among living American marsupials. The largest diploid number of chromosomes was 22, possessed by four genera of American marsupials, namely $D i$ delphis (three species), Chironectes (one), Lutreolina (one), and Philander (two). The other complements were $2 n=18$ in the short-tailed mouse opossum Monodelphis (three of about 15 species) and $2 n=14$ in Caluromys (three species), Caluromysiops (one), Marmosa (sensu lato; eight species of about 38, since rearranged into four genera), Metachirus (one), and Dromiciops (one). The $2 n=14$ karyotype is generally regarded as the primitive one. No reclassification of American marsupials was based on their cytogenetic constitution. In any case, Kirsch (1977) had already removed woolly opossums (Caluromys) from the Didelphidae and erected the family Caluromyidae for them. In 1992, Hershkovitz (1992a) established the family Marmosidae for most so-called mouse opossums previously known as Marmosa and raised Glironia to family rank. Four opossum

FIG. 1. Representatives of the four genera of large American marsupials usually referred to as the family Didelphidae, subfamily Didelphinae. Here only Didelphis marsupialis and Philander opossum are so classified. (Figures not to scale.) 
genera were retained in Didelphidae because they are the largest and their karyotype is $2 n=22$. These four genera are Didelphis, Chironectes, Philander of Linnaeus, and Lutreolina Thomas, 1910 (Figs. 1-3).

Biochemical, chromosomal, and anatomical characters were considered by Reig et al. (1985) in an attempt to determine the phylogenetic relationships among didelphoid marsupials. The extinct forms they considered are outside the scope of this monograph, as are most of the included genera. The Didelphidae of concern here include the three subfamilies Herpetotherinae, Caluromyinae, and Didelphinae. The latter was further subdivided into the tribes Didelphini, Metachirini, and Marmosini. Their tree (Reig et al., 1985, Fig. 2, p. 339) shows all American didelphoids and basic Australian forms emerging from Upper Cretaceous Microbiotheriidae, an improbability. It also shows didelphids arising in the Eocene and giving rise to the Marmosini with the primitive karyotype $2 n=14$ and pouchless reproductive system in the Oligocene-Miocene. This also seems unlikely. The pouchless and non-prehensile-tailed, terrestrial Metachirini with $2 n=14$ may have given rise to didelphids rather than the reverse as depicted.

Studies by Kirsch et al. (1993a) of DNA/DNA hybridization resulted in assignment of the five largest American genera (Didelphis, Philander, Lutreolina, Chironectes, and Metachirus) to the Didelphidae. Morphologically, as shown below, Lutreolina and Chironectes share few traits with each other and fewer with Didelphis, and the indicated position of Metachirus is even less tenable.

Patton et al. (1995 [1996]) regarded the systematic position of Metachirus to be somewhat equivocal:

While the brown four-eyed opossum Metachirus forms a sister taxon to the (Didelphis + Philander) clade in all trees, this relationship is not well supported. Bootstrap values for parsimony analyses are only 68 and 54 for DNA and amino acid sequences, respectively, and decay indices indicate that it would take only two or three additional steps to collapse the relationship between Metachirus and the other two genera. Similarly, confidence limits for this node are only $28 \%$ in the DNA neighbor-joining distance tree. The pouchless, primitively $2 n=14$ brown four-eyed opossum contrasts markedly with the pouched, derived $2 n=22$ Didelphis and Philander pair, although the three genera apparently do share similarities in bullar structure (Reig et al., 1987). Indeed, the total evidence Wagner tree presented by Reig et al. (1987, Fig. 59), based on 45 craniodental, cytological, sero- logical, and soft anatomical characters, does place Metachirus in a sister relationship with Didelphis and Philander, in contrast to Marmosa and Monodelphis, as do DNA-DNA hybridization data (Kirseh et al., 1995; Lapointe and Kirsch, 1995). Nevertheless, placements of Metachirus either as the sister of (Didelphis + Philander), with the mouse opossum lineage, or outside of both elades are all equally likely based on an evaluation of these alternative topologies by the log-likelihood test of Kishino and Hasegawa (1989). As a consequence, perhaps Metachirus is best considered as a basal member of the (Didelphis + Philander $)$ and ((Marmosa + Micoureus) + Monodelphis) clades. This is the same conclusion reached by Kirsch (1977) and Reig et al. (1987).

Returning to Philander, Patton et al. (1995 [1996]) do not explain why they dropped the prior name Philander andersoni Osgood, 1913, and consistently used instead its junior synonym, $P$. mcilhennyi Gardner and Patton, 1972, in all their discussions of marsupial relationships.

\section{A Morphological Test}

The four genera of large American marsupials have consistently been regarded as forming a natural assemblage within the family Didelphidae. This can be tested by the 30 morphological characters outlined below. Philander shares most characters with Didelphis. Chironectes and $\mathrm{Lu}$ treolina, however, have diverged widely from the preceding two genera and from a hypothetical common ancestor of all four. Two of the few shared characters (large size and $2 n=22$ ) may have evolved independently; other characters that may appear trivial are diagnostic nevertheless. An appraisal of the evolutionary stage of each character is shown in parentheses.

1. Largest of living New World marsupials (derived)

The four genera including the largest living New World opossums (Fig. 1) range in size from the smallest, Philander, through Chironectes and Lutreolina, to the largest, Didelphis. Size may have evolved independently in each genus, and its phylogenetic significance in the present context is dubious.

2. Karyotype $2 n=22$ (derived)

The same karyotype evolved independently in unrelated Marmosa canescens from a $2 n=14$ karyotype (Engstrom \& Gardner, 1988, p. 231). It also occurs in 


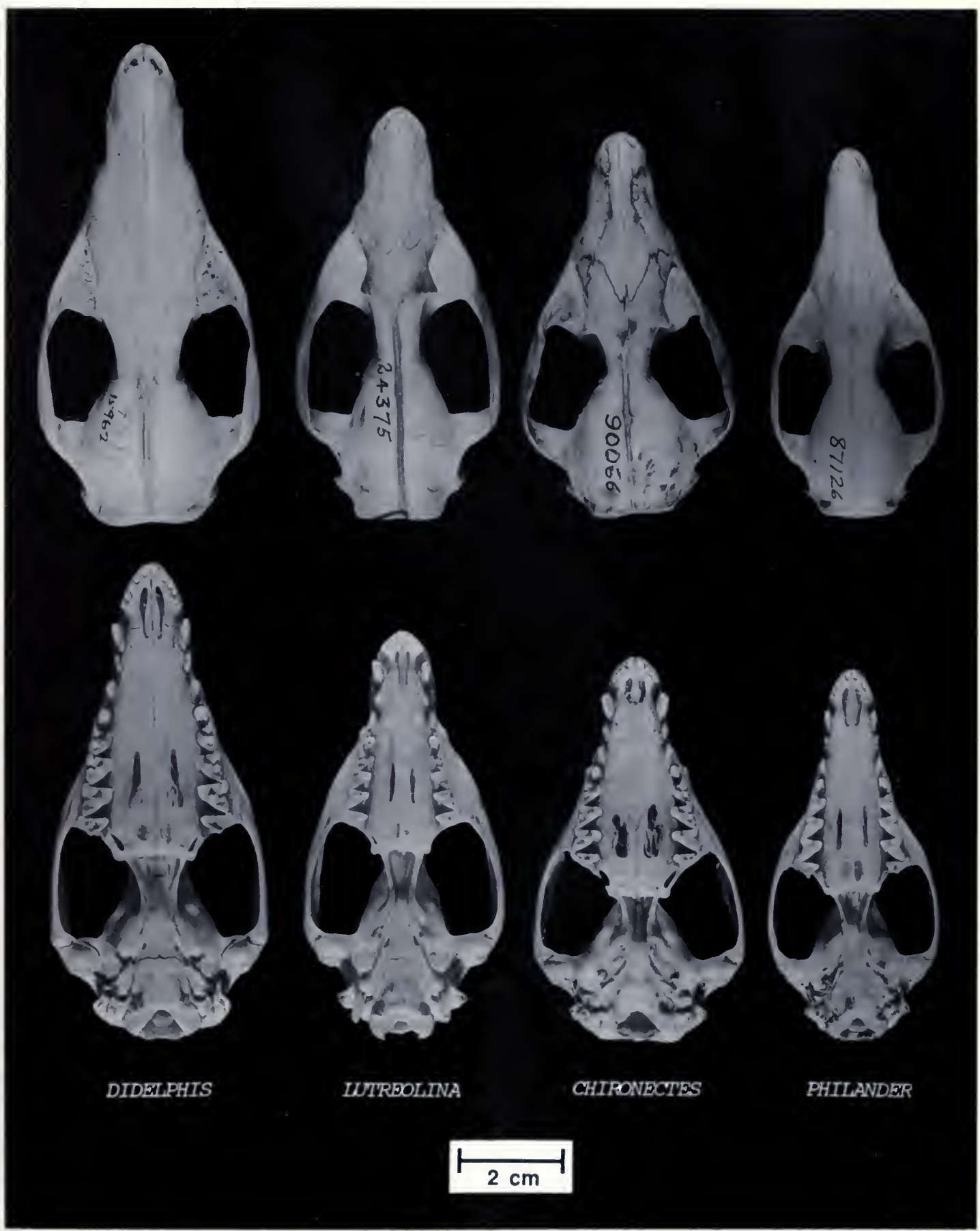

FIG. 2. Dorsal and ventral aspects of skulls of four large American marsupials (shown in order of decreasing size from left to right). 
Australian Macropodidae and Potoroidae. The phylogenetic significance of the same chromosome number in Chironectes and Lutreolina may be misleading. A different karyotypic pattern in Didelphis virginiana does not alter the fact that this species and D. marsupialis are very nearly alike and most intimately related.

3. Tail as long as or longer than head and body combined (incipiently derived)

Excludes Lutreolina

4. Tail fully prehensile (derived)

Excludes Lutreolina and Chironectes

5. Tail terete (plesiomorphic)

Excludes Lutreolina and Chironectes

6. Tail base not notably thickened (plesiomorphic)

Excludes Lutreolina (muscle, not fat)

7. Tail thinly pilose, the scales fully exposed dorsally (plesiomorphic)

Excludes Lutreolina

8. Complete marsupium present in females, absent in males (derived)

Excludes Lutreolina and Chironectes

9. Thumb and first toe fully opposable (derived)

Excludes Lutreolina

10. Pedal digits partially or not webbed (incipiently derived)

Excludes Chironectes

11. Crown color pattern whitish above eyes, brownish between (derived)

Excludes Chironectes and Lutreolina

12. Ears large, roundish, leaflike, and when laid forward extending midway or more to outer canthus of eye (possibly derived)

Excludes Lutreolina and Chironectes

13. Manual ungues sharp and protruding (plesiomorphic)

Excludes Chironectes

14. Paired posteromedian palatal vacuities present (plesiomorphic)

Excludes Chironectes

15. Premaxillary symphysis angular (plesiomorphic)

Excludes Lutreolina and Chironectes

16. Depression at proximal end of temporal ridges (?)

Excludes Lutreolina and Chironectes

17. Interorbital region not notably constricted (derived?)

Excludes Lutreolina

18. Supraorbital processes rudimentary (incipiently derived)

Excludes Lutreolina and Chironectes
19. Spread of zygomatic arches comparatively narrow (plesiomorphic)

Excludes Chironectes

20. Lacrymal foramina facial (derived)

Excludes Lutreolina and Chironectes

21. Nasals behind convergent to a single point or with slight spread between points (plesiomorphic?)

Excludes Lutreolina and Chironectes

22. Maxillary sheath for insertion of lower canine shallow (plesiomorphic)

Excludes Lutreolina and Chironectes

23. Occipital condyles projecting behind vertical plane of supraorbital bone (plesiomorphic)

Excludes Chironectes

24. Incisive foramina long, narrow, and tapered (plesiomorphic)

Excludes Chironectes

25. Foramen magnum ovate (?)

Excludes Lutreolina

26. Ventral mandibular plane between canine and incisors horizontal (plesiomorphic)

Excludes Lutreolina and Chironectes (?)

27. Upper incisors $2-5$ with little or no deflection inward and upward (plesiomorphic?)

Excludes Lutreolina and Chironectes

28. $\mathrm{P}^{2}$ nearly as long as $\mathrm{P}^{3}$ (plesiomorphic?)

Excludes Lutreolina

29. Lower incisors not overlapping (staggered $\mathrm{i}_{3}$ not included) (plesiomorphic)

Excludes Lutreolina and Chironectes

30. Locomotion terrestrial-scansorial (derived) Excludes Lutreolina and Chironectes

Taking into account other characters mentioned beyond, comparisons reveal Didelphis as a shaggy, oversized "Philander." The otter-like Chironectes, distinguished mainly by its nonprehensile, naked tail, with both sexes pouched, and the terrestrial weasel-like Lutreolina, with its nonprehensile, completely hairy tail and nonopposable first digit, have radiated far from didelphoid roots. Lacking affinities with each other of didelphid grade, Chironectes and Lutreolina are each treated as a type of a distinct subfamily. The new arrangement follows:

Superfamily Didelphoidea

Family Didelphidae Gray, 1821

Subfamily Didelphinae

Genus Philander Tiedemann, 1808

Philander opossum Linnaeus, 1758

Philander andersoni Osgood, 1913 


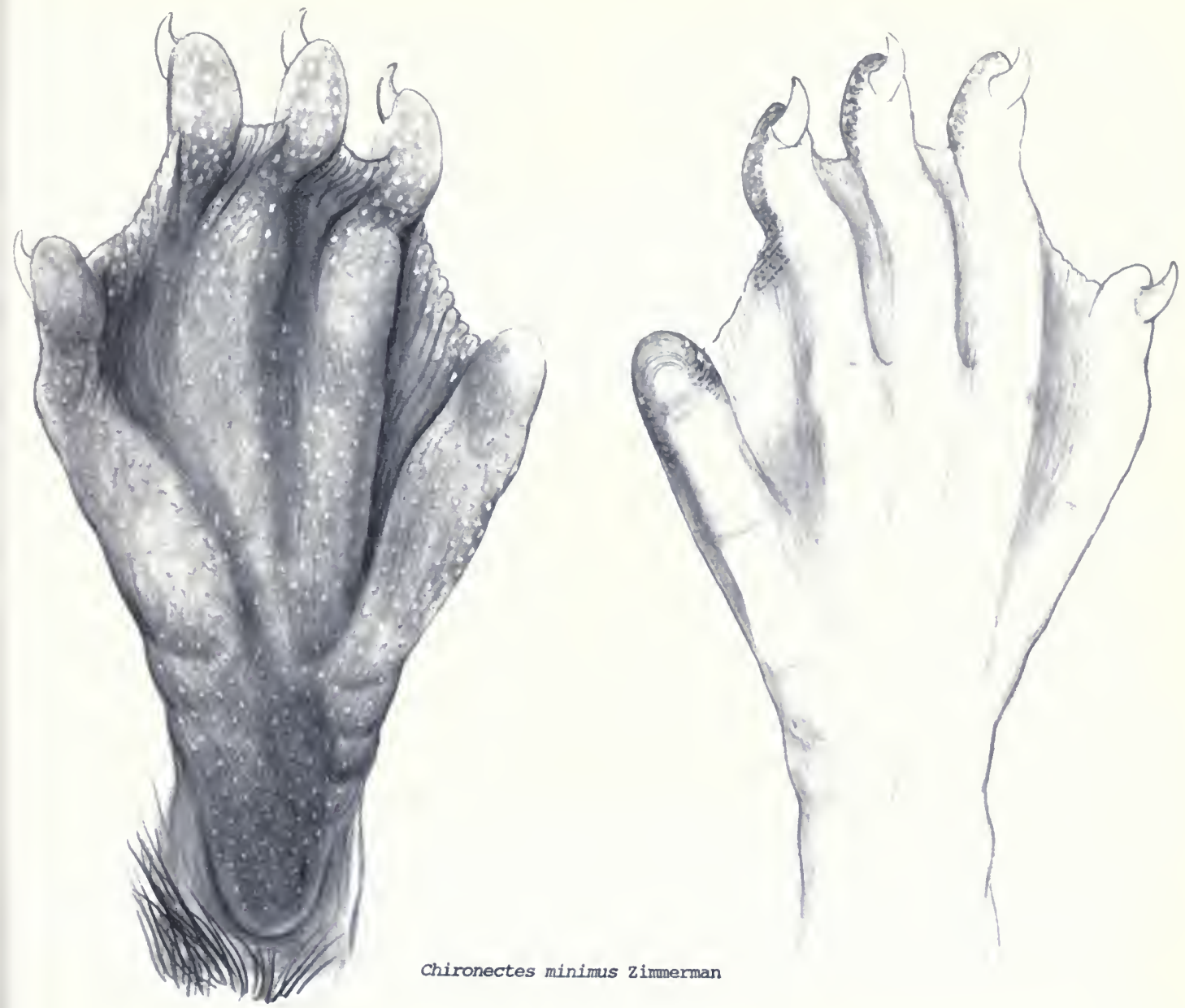

Fig. 3. Chironectes minimus Zimmerman. Plantar and dorsal surfaces of fully webbed right hindfoot.

Genus Didelphis Linnaeus, 1758

Didelphis marsupialis Linnaeus, 1758 (aurita Wied-Neuwied a synonym)

Didelphis albiventris Lund, 1840

Didelphis virginiana Kerr, 1792

Subfamily Chironectinae (new)

Genus Chironectes Illiger, 1811

Chironectes minimus Zimmermann, 1780

Subfamily Lutreolininae (new)

Genus Lutreolina Thomas, 1910

Lutreolina crassicaudata

Desmarest, 1804

Didelphis is the best known and most widely distributed genus of American marsupials. Its northernmost species, D. virginiana, occurs in North and Middle America; the more southern D. marsupialis, in México and Middle and South America; and $D$. albiventris, in South America. The North American representatives of $D$. virginiana and $D$. marsupialis have been taxonomically revised by Gardner (1973).

Distinctive characters of the family and subfamilies are explicit in each of the 30 characters described above. Detailed accounts of Chironectes and Lutreolina await formal taxonomic revision such as given below for Philander. Didelphis is highly derived. Its roots in the past are unknown. The animal that evolved a prehensile tail, opposable first digit, complete marsupium in stages, and other derived characters is far removed in time from Didelphis. It is most unlikely 
that the genus Didelphis existed as such in the Early Tertiary or even Late Tertiary. To refer to a staggered-lower-incisored Didelphis as the stock from which the Early Paleocene Bolivian microbiothere Pucadelphys andinus Marshall and de Muizon (1988) could be derived is anachronistic and morphologically perverted.

A taxonomic revision of Philander, the remaining genus of the Didelphinae, follows. Comparisons are made with Didelphis, Chironectes, and Lutreolina. Except for an occasional reference here, Metachirus is dealt with in a separate paper that is in preparation.

\section{Genus Philander Tiedemann (Four-Eyed Pouched Opossums)}

Philander Brisson, 1762:13, 207-214_name from non-Linnaean work and not available. Gilmore, 1941:316-Metachirops Matschie antedated; characters; distribution; yellow fever susceptibility. Hopwood, 1947:533_names from Brisson not valid; Philander Gronovius non-Linnaean and not available.

Philander Tiedemann, 1808:426-included species $P$. virginianus Tiedemann [ $=D$. opossum Linnaeus], P. murinus [ = Marmosa murina], P. brachyurus [= Monodelphis brachyura]. Hershkovitz, 1949: 11 -type $P$. virginianus Tiedemann $=$ Didelphis opossum Linnaeus; Philander Brisson, 1762, and Philander Gronovius, 1763, not valid. Hildebrand, 1961:239 - comparative body proportions. Perondini and Perondini, 1965:381, Fig. 7 (metaphase chromosomes), Fig. 8 (karyogram)—chromosome complement $2 n=22$. Enders, 1966:195-breeding season; habits. Biggers, 1966:251—male external genitalia; spermatozoan types; temperature regulation. Enders and Enders, 1969:431, Pl. 1 (uterus with 5 fetuses), Pls. 2-6 (uterus section)_placenta; fetal membranes; fetal size at birth. Hayman and Martin, 1969:192 - chromosomes $(2 n=22)$; chromosome phylogeny. Collins, 1973:69—care and maintenance in captivity; reproduction; growth and development; diet; parasites. Tyndale-Biscoe, 1973:25, 38, 40, 61, 65, 189, 192, 230-biology. Pine, 1973:391—not the four-eyed pouched opossum. Hershkovitz, 1976:295-history, nomenclature. McNab, 1978:115-comparative bioenergetics ( $P$. opossum). Husson, 1978:27-treated as synonym of Didelphis Linnaeus by designation of the Virginia opossum as lectotype of type species $P$. virginianus Tiedemann. Landsmeer, 1979:337, Figs. 1, 4, 11, 12 (cheiridia, extensor assemblies)digital extensor muscles; claw retraction. Hersh- kovitz, 1981:943-Philander valid generic name; type $P$. virginianus Tiedemann $=$ Didelphis opossum Linnaeus. Gardner, 1981:447-Philander valid; Husson opinion rejected. Rodger, 1982:270testis and excurrent ducts; paired spermatozoa. Jenkins and Knutson, 1983-type specimens in British Museum (Natural History). Pérez-Hernández, 1985:53, 65-generic characters. Pérez-Hernández et al., 1986:14 dental morphology and diet. Gardner (in Wilson \& Reeder, eds.), 1993:22-synonymy; species ( $P$. opossum, $P$. andersoni) distribution.

Metachirops Matschie, 1916:262, 267, 268-included species pallidus Allen, fuscogriseus Allen, grisescens Allen, melanurus Thomas, opossum Linnaeus, canus Osgood, andersoni Osgood, quica Temminck (designated type), frenata Lichtenstein; taxonomy. Miranda Ribeiro, 1936:340 — characters. Krumbiegel, 1941:199-review. Pine, 1973:391valid generic name for four-eyed pouched opossum; Philander regarded a synonym of Didelphis.

Holothylax Cabrera, 1919:47-type Didelphis opossum Linnaeus by original designation.

Metachirus Burmeister, 1854:135-subgenus of Didelphys [sic] Linnaeus, part, D. quica Temminck only. Burmeister, 1856:68-part, $M$. opossum Linnaeus only. Thomas, 1888:329-part, D. opossum Linnaeus only. Sonntag, 1924:743-comparative tongue anatomy ( $M$. opossum). Hill and Fraser, 1925:196, Pl. 1, Fig. 3 (urogenital organs), Pl. 4, Figs. 15-17 (vagina)-female urogenital system. Boardman, 1952:848 - hair tracts ( $M$. opossum). Lyne, 1959:84-vibrissae (M. opossum).

Metacheirus [sic] Sanderson, 1949:787-misspelling of Metachirus, in combination with $M$. opossum.

TYPE SPECIES-Philander virginianus Tiedemann $=$ Didelphis opossum Linnaeus.

\section{Key to the Species (see also Fig. 4)}

Dorsum uniformly dark or grayish, blackish middorsal stripe absent or poorly defined ........ ................... P. opossum (p. 33) Dorsum with well-defined blackish middorsal stripe or band ......... P. andersoni (p. 33)

Distribution-Figure 5. Tropical and subtropical forests including second growth, in South and Middle America, from Misiones and Chaco in Argentina, and in Paraguay east of the Río Paraguay, northward into eastern Brasil as far as Bahia on the southeast, west from the Río Tocantins-Araguaia into Bolivia, and Perú north into Ecuador, Colombia, Venezuela, the Guianan countries, Panamá, Costa Rica, Nicaragua, Honduras, Salvador, Guatemala, Belize, and in México, the state of

Fig. 4. Pigmentation of dorsum. A, Philander opossum; B, P. andersoni andersoni; C, P. andersoni mcilhennyi. 


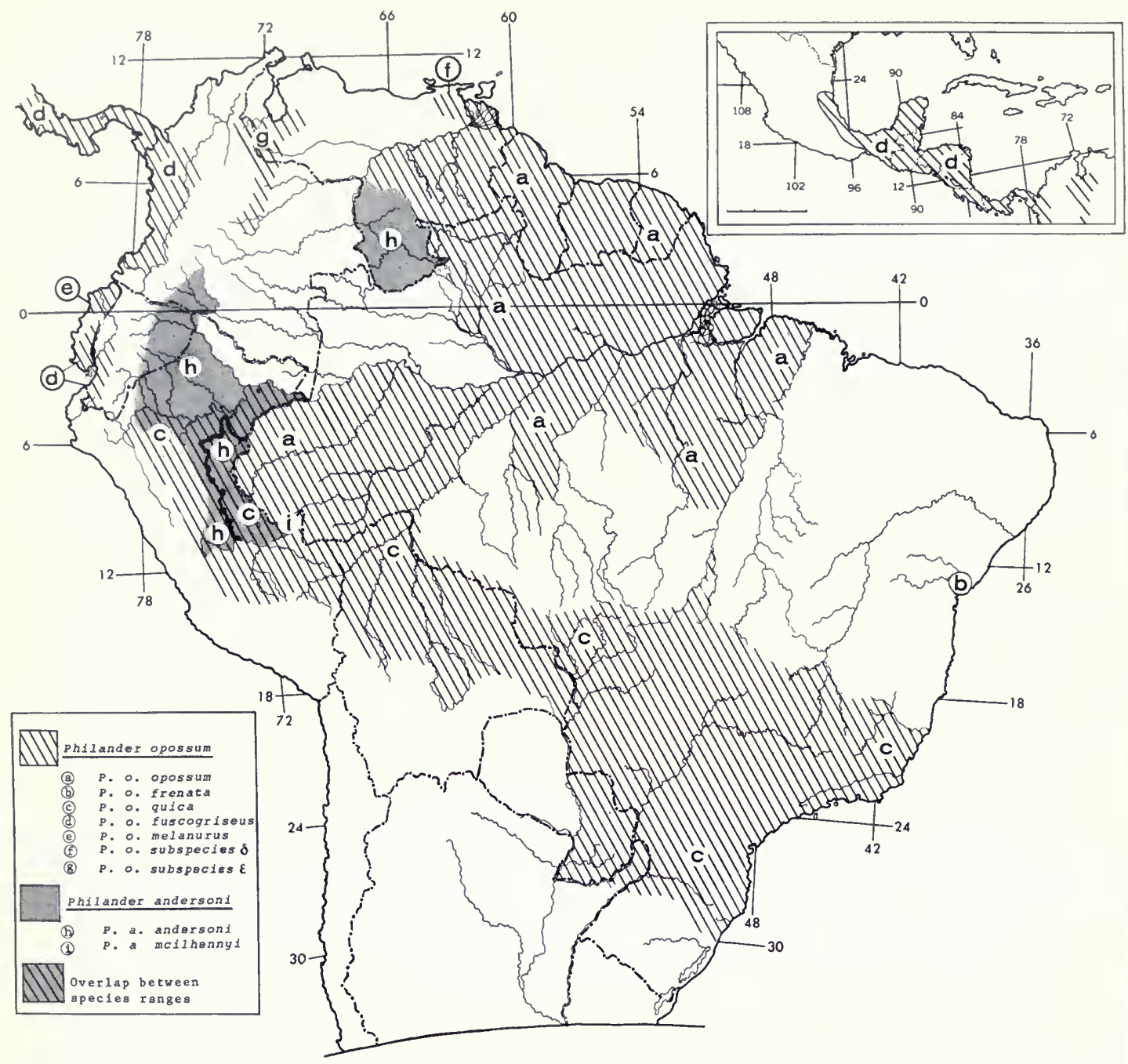

FIG. 5. Distribution of subspecies of Philander opossum (a-g), P. andersoni (h), and P. andersoni mcilhennyi (i) in South America and Middle America (part inset).

Tamaulipas on the Atlantic versant, Oaxaca on the Pacific; altitudinal range from sea level to about $2000 \mathrm{~m}$ above.

Philander is unknown in the Caribbean islands, the Caribbean coast of Venezuela and Colombia, and the inland highlands (tepuis) of Venezuela. The genus has not been recorded from the Sierra Nevada de Santa Marta and the northwestern lowlands in Colombia. Spotty records from the Brasilian Amazonas suggest that Philander may be present throughout the basin east of the Río Negro and east of the Río Tocantins-Araguaia. Although Philander is generally absent from the drier parts of the cerrado of central Brasil, it does occur in the gallery forests. The habitats are described in greater detail in the species and subspecies accounts.

\section{Nomenclature}

Tiedemann $(1808$, p. 426$)$ included three nominal didelphid species in his Philander. The first of these is "Virginische opossum, P[hilander]. virginianus (Did. opossum L[innaeus])" and is based on bibliographical references to figures of opossums by Buffon (1763) and Schreber (17391810) and anatomical accounts by Tyson (1698) 
and Cowper (1704). His diagnosis, "Korper rothlich braun. Ueber jedem Augen ein gelbich weiser flecken. Schwanz so lang als der Leib. I Fuss and 3 Zoll lang ohne den Schwanz," is of a four-eyed opossum, and Tiedemann's use of Didelphis opossum Linnaeus in apposition definitely restricts the name to the pouched four-eyed species. The other two species are "P. murinus (Did. murina L.)," a Marmosa, and "P. brachyurus (Did. brachyuros Penn.)," a Monodelphis.

Philander Tiedemann derives ultimately from Seba's (1734) vernacular names, "Philander, opossum s. Carigueja," cited by Linnaeus (1758) in his description of D[idelphis]. opossum. Philander virginianus, a substitute name for $D$. opossum Linnaeus, is type by subsequent designation (Hershkovitz, 1949, p. 11).

Didelphis philander Linnaeus, 1758, designated type by Thomas (1888, p. 336), was not included in the original erection of the genus Philander and hence is not valid as type.

Pine (1973, p. 391) and Husson (1978, p. 27) argued against the use of Philander Tiedemann for the four-eyed pouched opossum and elected Metachirops Matschie, a junior synonym, instead. The arguments of both authors were discussed and rejected by Hershkovitz (1976; 1981) and by Gardner (1981, p. 44), who summarized and laid the dispute to rest.

\section{External Characters and Comparisons}

BODY SIZE AND Form-Species of the genus Philander average smaller than those of Didelphis, Lutreolina, and Chironectes but larger than all other living didelphoids. Average combined head and body length is about $281 \mathrm{~mm}$ (slightly less in females than males); condylobasal length of skull, an indicator of body size, is about 72 $\mathrm{mm}$. Trunk is elongate; muzzle long and slender; eyes large; ears conspicuous. Philander andersoni averages slightly larger than $P$. opossum, and geographically peripheral races of each species average larger than more central ones.

\section{MEASUREMENTS-Table 1.}

GrowTH-Body mass increases after full eruption of all molars. Largest individuals of a series or subspecies, however, are not necessarily oldest and may include individuals classified as subadult on the basis of dental eruption or wear and suture closure. Males may not be larger than females at birth but evidently outgrow females to maturity.
Adult wild-caught animals that had been maintained in cages and fed without stint in the now defunct Rockefeller Laboratories in Rio de Janeiro became extremely large in body mass and cranial length. Dental size, however, fixed at complete eruption, remains the reliable indicator of "normal" body size.

Basic Coloration (Fig. 4)-Trunk dark grayish or buffy agouti with or without a blackish middorsal stripe or band; face dark brown or blackish with contrasting supraorbital spot, rhinarial tip usually unpigmented; tail dark brown or with underside slightly paler, terminal half less pigmented or unpigmented.

The Philander color pattern of a dark, modified agouti coat, dark brown to buffy head, dark brown tail and ears, could give rise to all colors and color combinations present in didelphoids, including Didelphis, Lutreolina, and Chironectes. A dark brown eye ring is characteristic of nearly all didelphoids, and supraorbital spots such as those of Philander expand into temporal bands in Chironectes and the whitish face of many Didelphis. The more or less pigmented ears and tails of didelphoids derive from fully pigmented ones. The saturate, bleached, or whitish cover hairs evolved from agouti cover hairs. Blackish (melanistic), grayish, orange, or reddish phases, whitish guard hairs and wool hairs, can be derived from the basic color pattern of $P$. opossum. Coloration of body and limbs, tail, and ears of Lutreolina and the brindled body coloration of Chironectes can also be derived from a primitive Philander model. The saturate, pheomelanic facial pattern of $\mathbf{L u}$ treolina, without markings, however, is rare among didelphoids. The nearest common ancestral facial pattern of both Lutreolina and Philander must have been nearly uniformly modified agouti.

COlor Phases - A "gray" or grayish brown phase and a "brown," orange-brown, or orange phase occur in both species of Philander. In the gray phase, the subterminal or pheomelanic bands of the cover hairs of dorsum and sides are bleached to pale buff or nearly colorless. The general effect is a grayish brown appearance. In the brown phase, the subterminal bands are more densely pigmented, giving the animal an ochraceous or orange appearance. Hairs of underparts in gray-phase individuals are usually bicolor with the dark eumelanic bases showing through. In brown-phase individuals, the ventral hairs are usually or predominantly pheomelanic to roots.

The color term "brown," used by Mustrangi 
TABLE 1. Summary of measurements of subspecies of Philander opossum and subspecies of P. andersoni. Measurement means (extremes in parentheses) and number of specimens.

\section{Head and Body}

Philander opossum opossum Linnaeus

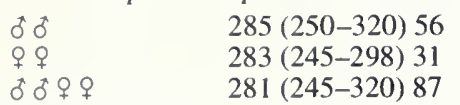

Philander opossum quica Temminck

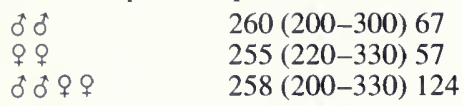

Philander opossum melanurus Thomas

\begin{tabular}{|c|c|c|}
\hline $\begin{array}{l}6 \\
6 \\
309\end{array}$ & $\begin{array}{l}272(258-296) 7 \\
284,282 \\
274(258-296) 9\end{array}$ & $\begin{array}{l}264(250-276) 7 \\
270,229 \\
261(229-276) 9\end{array}$ \\
\hline
\end{tabular}

Philander opossum fuscogriseus J. A. Allen

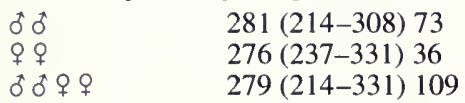

Philander andersoni andersoni Osgood

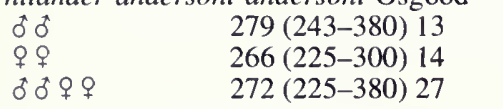

Tail

$$
\begin{aligned}
& 290(235-343) 55 \\
& 285(245-350) 31 \\
& 288(245-350) 86
\end{aligned}
$$

$292(195-355) 63$
$279(220-310) 55$
$291(195-355) 118$

$289(240-335) 73$
$284(240-320) 36$
$287(240-335) 109$

$284(255-315) 13$

$282(255-332) 14$

$283(255-332) 27$
Hind Foot

$43(35-50) 55$

$40(35-43) 25$

$42(35-50) 81$

$40(29-48) 64$

$38(32-46) 53$

$39(29-48) 117$

$40(37-43) 7$

35,37

$39(35-43) 9$

$45(37-52) 72$

$42(37-50) 36$

$44(37-52) 108$

$42(35-48) 15$

$41(35-51) 14$

$41(35-51) 29$

43,40
$41(36-44) 5$
$41(36-44) 7$
Ear

$36(30-42) 21$

$32(30-41) 10$

$35(30-42) 32$

$34(22-42) 44$

$33(22-43) 46$

$34(22-43) 90$

$29(27-35) 7$

18,32

$28(18-35) 9$

$36(32-40) 57$

$37(25-40) 28$

$36(25-40) 85$

$37(30-45) 13$

$36(33-41) 12$

$37(30-45) 25$

Philander andersoni mcilhennyi Gardner and Patton

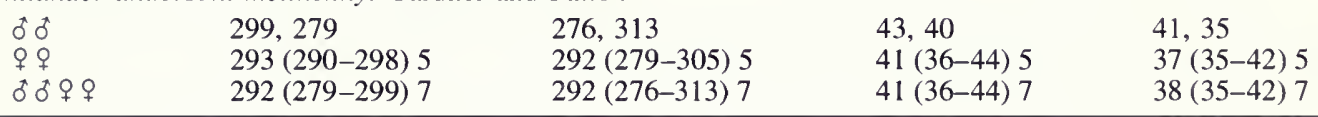

(1994, p. 252) to describe a pale color phase of Marmosops incanus Lund (= M. scapulatus Burmeister), may be a tone of pheomelanin, or reddish. True melanin, or "blackish," is actually brown. The female Philander I captured in the Parque Nacional do Caparaó was reported by Mustrangi (1994, p. 253), who happened to see the prepared specimen while it was en route to Chicago for my study. She described it as of "a similar, pale coloration" as the Marmosops incanus of her report. The pheomelanic or erythristic color phase and the melanistic phase are rare mutants among mammals and birds generally (cf. Hershkovitz, 1968; 1970; 1977; 1992a).

Pelage-Fur short in $P$. opossum, longer in $P$. andersoni, particularly in $P$. andersoni mcilhennyi, in which middorsal guard hairs are predominant. Downy wool hairs are preeminent in the aquatic Chironectes. Didelphis pelage is shaggy, the long guard hairs and wool hairs about equally conspicuous and almost entirely concealing the cover hairs. Pelage of Lutreolina is short with underfur, the silky guard hairs weak. A pelage like that of
P. opossum is common among didelphoids and could give rise to any of the more derived types.

MoLT-Changes in pelage with age or season cannot be accurately determined on the basis of material at hand. The few available large series of Philander opossum, such as the 85 specimens (AMNH) from Ilha Taiuna, Pará, Brasil, collected between 31 October and 11 November 1931, provide no definite information on seasonal molt and very little regarding ontogenetic change. Adults in both old and new pelage without visible molt line may occur at the same time in the same general area. The fine, thin juvenal pelage is gradually replaced by coarser, thicker adult pelage. Juvenal coloration is like that of adults.

LimB Bones AND Locomotion (Table 2)-Using condylobasal length as an indicator of overall body size, the cranial length ranges from about 29 $\mathrm{mm}$ in Dromiciops gliroides to about $112 \mathrm{~mm}$ in Didelphis marsupialis, a differential of about 85 $\mathrm{mm}$. The hind limb is longer than the forelimb in all taxa, and the tibia about as long as, or usually longer than, the femur. In the forelimb, the radius 
TABLE 1. Extended.

\begin{tabular}{|c|c|c|c|c|}
\hline $\begin{array}{c}\text { Condylobasal } \\
\text { Length }\end{array}$ & $\begin{array}{l}\text { Zygomatic } \\
\text { Breadth }\end{array}$ & $\begin{array}{l}\text { Preorbital } \\
\text { Width }\end{array}$ & $\begin{array}{l}\text { Postorbital } \\
\text { Width }\end{array}$ & $\begin{array}{l}\text { Braincase } \\
\text { Width }\end{array}$ \\
\hline $73.0(65.4-82.1) 75$ & $38.3(33.0-43.4) 73$ & $14.1(11.5-18.9) 59$ & $8.8(7.5-9.7) 74$ & $21.2(18.4-24.0) 72$ \\
\hline $69.7(64.0-80.8) 39$ & $34.5(31.1-40.7) 34$ & $13.1(11.4-18.4) 23$ & $8.7(8.0-9.7) 34$ & $19.9(18.9-21.4) 35$ \\
\hline $71.9(64.0-82.1) 114$ & $37.0(31.1-43.4) 107$ & $13.8(11.4-18.9) 82$ & $8.8(7.5-9.7) 108$ & $20.8(18.4-24.0) 107$ \\
\hline $66.4(60.0-76.4) 78$ & $35.5(30.7-43.7) 75$ & $12.6(10.8-15.7) 36$ & $8.4(7.4-9.1) 77$ & $20.2(18.2-23.1) 71$ \\
\hline $62.8(57.1-72.4) 76$ & $31.1(28.0-38.3) 67$ & $11.6(9.7-14.7) 42$ & $8.4(7.5-9.9) 67$ & $19.7(18.0-21.9) 67$ \\
\hline $64.6(57.1-76.4) 154$ & $33.4(28.0-43.7) 142$ & $12.1(9.7-15.7) 78$ & $8.4(7.4-9.9) 144$ & $20.0(18.0-23.0) 138$ \\
\hline $69.6(64.6-71.9) 8$ & $36.6(33.8-38.1) 8$ & $13.2(12.3-13.9) 6$ & $9.2(8.7-9.7) 8$ & $21.5(20.4-23.5) 8$ \\
\hline $64.1,65.8$ & $33.5,34.2$ & -12.5 & $9.4,8.4$ & $20.9,19.3$ \\
\hline $68.7(64.1-71.9) 10$ & $36.0(33.5-38.1) 10$ & $13.1(12.3-13.9) 7$ & $9.1(8.4-9.7) 10$ & $21.2(19.3-23.5) 10$ \\
\hline $72.7(61.9-81.5) 76$ & $38.4(32.2-43.0) 73$ & $13.4(10.9-15.7) 61$ & $9.0(8.3-9.5) 73$ & $21.0(19.1-23.5) 75$ \\
\hline $69.7(60.0-80.5) 41$ & $36.1(30.7-43.1) 40$ & $12.9(11.5-16.6) 31$ & $8.9(8.0-9.4) 40$ & $20.6(18.7-23.6) 40$ \\
\hline $71.6(60.0-81.5) 117$ & $37.6(30.7-43.1) 113$ & $13.2(10.9-16.6) 92$ & $8.7(8.0-9.5) 113$ & $20.9(18.7-23.6) 115$ \\
\hline $72.3(66.7-76.5) 17$ & $37.1(32.3-39.9) 17$ & $13.6(11.8-16.2) 13$ & $9.3(8.0-9.4) 17$ & $21.2(19.6-22.6) 16$ \\
\hline $68.9(64.6-81.2) 17$ & $34.1(30.8-40.0) 17$ & $12.6(10.4-14.0) 13$ & $8.8(8.0-10.1) 18$ & $20.6(19.3-23.9) 17$ \\
\hline $70.6(64.6-81.2) 34$ & $35.6(30.8-40.0) 34$ & $13.1(10.4-16.2) 26$ & $9.1(8.0-10.1) 35$ & $20.9(19.3-23.9) 33$ \\
\hline $74.4,73.1$ & $37.6,36.5$ & $14.0,13.5$ & $9.0,8.4$ & $21.2,20.8$ \\
\hline $71.8(70.2-73.9) 4$ & $35.8(34.2-36.8) 4$ & $13.7(12.7-14.7) 4$ & $9.0(8.7-9.2) 4$ & $20.7(20.2-21.0) 4$ \\
\hline $72.5(70.2-74.4) 6$ & $36.2(34.2-37.6) 6$ & $13.7(12.7-14.7) 6$ & $8.9(8.4-9.2) 6$ & $20.8(20.2-21.2) 6$ \\
\hline
\end{tabular}

is about as long as the humerus or shorter. In the semiaquatic Chironectes it averages less.

The hind limb is longer than the forelimb in the Marmosidae, with the greatest differential shown by the Patagonian Lestodelphys halli, apparently a leaper or springer. It is closely followed in limbto-trunk proportions by the terrestrial Metachirus mudicaudatus. No American marsupial is a burrower. Chironectes is the only living American marsupial adapted for aquatic life, but all American marsupials are good swimmers and divers, except possibly the Marmosidae. All are habitual tree climbers, except Lutreolina, Metachirus, Chironectes, Lestodelphys, and Monodelphis, all of which probably can climb but have not been observed doing so.

The long hind limbs of Philander are well adapted for running and springing. The proportionately shorter limbs of Didelphis, especially the anterior, are better specialized for climbing. Animals of both genera progress with facility on the ground, Philander with moderate to rapid speed and Didelphis slowly and with a lumbering gait that does not exclude a swift sprint by a frightened animal. Chi- ronectes is preeminently adapted for aquatic life, its long legs and palmate hind feet serving for propulsion through water, the gritty palms and digits of the front feet for seizing slippery prey and climbing slippery rocks and boulders in mountain streams. Limbs of Lutreolina are short and weasellike and apparently better adapted for terrestrial locomotion than for climbing or swimming.

Cheiridia (Fig. 6) - Hands and feet of Philander are modified for grasping, the pollex and hallux opposable, the latter inunguis; fifth hind toe much shorter than fourth, three middle digits subequal; toes not webbed. Manual and pedal claws of Didelphis stout, hallux inunguis, opposable; claws sharp, recurved; digits of Lutreolina as in Didelphis but claws blunt, little recurved. In Chironectes manual digits with short needle-like claws, hind feet with toes webbed, hallux inunguis (Fig. 3).

TAlL (Fig. 7)-Tail in Philander and Didelphis is cylindriform, tapered, prehensile, scaly, practically bare except for densely furred basal $3-9 \mathrm{~cm}$; naked ventrally, the terminal portion plicate between vertebral joints to permit clasping, length 
Palatal

Length $\mathbf{i}-\mathbf{m}^{4}$

$\mathbf{m}^{1-4}$

Philander opossum opossum Linnaeus

$$
\begin{array}{ll}
\text { ㅎํ } & 44.0(40.3-49.6) 75 \\
\text { 우 } & 41.9(38.4-45.8) 35 \\
\text { ㅎํㅇํ } 9 & 43.3(38.4-49.6) 110
\end{array}
$$

Philander opossum quica Temminck

$$
\begin{aligned}
& \text { o o } \quad 38.3(34.1-44.8) 75 \\
& \text { 우 } \quad 39.2(35.1-44.6) 71 \\
& \text { 훙ㅎㅇㅇㅜ } \quad 38.7(34.1-44.8) 146
\end{aligned}
$$

\begin{tabular}{|c|c|}
\hline & $43.4(39.7-48.9) 20$ \\
\hline & $42.0(39.7-49.6) 18$ \\
\hline రิq & $42.8(39.7-49.6) 38$ \\
\hline
\end{tabular}

Philander opossum melanurus Thomas

$$
\begin{aligned}
& \text { ơ } 042.0(38.6-43.1) 8 \\
& \text { 우아 } \\
& \text { бㅎㅎ우우 } \\
& 38.8,39.9 \\
& 41.4(38.6-43.1) 10
\end{aligned}
$$

Philander opossum fuscogriseus J. A. Allen

$$
\begin{aligned}
& \text { के } \quad 44.2(37.9-50.2) 74 \\
& \text { 우 } \\
& \text { ธิ㇒? } 9 \text { 우 } \\
& 42.5(37.0-48.7) 41 \\
& 43.6(37.0-50.2) 115
\end{aligned}
$$

Philander andersoni andersoni Osgood

Philander andersoni mcilhennyi Gardner and Patton

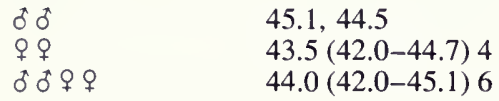

about equal to that of combined head and body; rhomboidal scales arranged spirally; the interscutular bristles usually 5 per scale (Fig. 8 ). The $D i$ delphis tail is more strongly prehensile than that of Philander, and that of the aquatic Chironectes is hardly if at all prehensile, its flattened ventral surface serving as a rudder. The Lutreolina tail is the most densely hirsute of the group. Length slightly less than head and body combined and, as in Philander, may be nearly the primitive proportion for mammals generally. The tails are not fat storing, but that of terrestrial Lutreolina is notably thick at base, where the transitional vertebrae provide insertion for large muscle mass (Fig. 1). (More on locomotion in Philander on p. 75.)

Transitional Caudal Vertebrae (Table 3)Transitional vertebrae of the postcranial skeleton, usually 4 or 5 in American marsupials, sometimes 6 depending on interpretation, are present between the sacrum and typical caudal (coccyxial) vertebrae. Although well differentiated (Fig. 9), they are usually counted as caudal. The morphological characters of the transitional vertebrae, however, are well marked, with the first distinct
$38.0(34.9-41.5) 75$

$36.8(34.8-39.3) 35$

$37.6(34.8-41.5) 110$

$34.4(32.0-37.7) 78$

$32.9(30.5-38.0) 71$

$34.2(30.5-38.0) 147$

$36.5(34.7-37.5) 8$

$34.6,34.8$

$36.2(34.6-37.5) 10$

$38.1(34.3-41.8) 77$

$36.9(33.9-41.2) 42$

$37.7(33.9-41.8) 119$

$38.0(35.2-43.3) 20$

$36.9(34.2-42.9) 18$

$37.5(34.2-43.3) 38$

$39.8,38.4$

$38.1(36.4-39.7) 4$

$38.5(36.4-39.8) 6$
$13.8(12.8-14.9) 75$

13.7 (12.7-14.7) 36

$13.8(12.7-14.9) 111$

$12.7(11.6-14.4) 80$

12.5 (11.2-14.5) 78

$12.6(11.2-14.5) 158$

$13.6(13.3-14.0) 8$

$13.0,13.0$

$13.5(13.0-14.0) 10$

$14.2(11.7-15.6) 77$

$13.9(12.8-15.3) 42$

$14.1(11.7-15.6) 119$

$13.9(12.0-16.0) 20$ $13.8(12.6-15.0) 18$ $13.8(12.0-16.0) 38$

$15.3,14.6$

$14.4(13.7-15.3) 5$

$14.6(13.7-15.3) 7$ from the sacrum, and the 5th (or 6th) usually sharply differentiated from the succeeding caudal vertebra. Muscles of the transitional vertebrae move the tail in particular directions. The action of a specific muscle may be narrowly restricted, such as that controlling movements of the tail tip.

EXTERNAL EAR (Fig. 1)-The auricular lamina of Philander is large, rounded, leafy or membranous and plicate, entirely blackish or with base of middle portion to total unpigmented; length, when laid forward, reaching outer canthus of eye. External ears of Didelphis and Chironectes are similar. The Lutreolina auricular lamina is much smaller, less leafy, hairy, and hardly if at all plicate. Auricles of the group could have evolved from a type with lamina little expanded, bare, and perhaps slightly motile or plicate. Didelphoid auricles are comparable to those of many species of shrews, lemurs, and callitrichids (cf. Hershkovitz, 1977, pp. 102-106).

MARSUPIUM-A fully formed pouch characterizes sexually mature females of Philander and $D i$ delphis and both sexes of the semiaquatic Chironectes. The pouch opens from the side in the first 
two, posteriorly in the female of the third, and medially in the male, in which it seals the scrotum during aquatic submersion. In Lutreolina the rudiment of a pouch is formed by a longitudinal skin fold on each side of the abdominal milk gland field.

Marsupium and scrotum are not homologous. They may be derived, however, from different parts of the same anlage (Tyndale-Biscoe \& Renfree, 1987, p. 121).

A pouch consists of a pair of recessed longitudinal skin folds, one on each side of the midabdominoinguinal region. The folds are united caudally but are separate anteriorly. Only in Didelphis does the inner margin of each lateral fold appose the other to form a flexible but completely closed waterproof pocket for shielding suckling young (cf. Enders, 1937, p. 25; 1966, p. 200).

Nipples (Table 4)-The well-developed marsupium of Philander conceals two rows of nipples arranged symmetrically, one on each side of the ventrum, and a median row with but one teat, the total number odd. The usual number of nipples, often called teats or mammae, is 7. Enders (1935, p. 411) found only 5 in an unspecified number of live-trapped Panamanian opossums and a maximum of 5 pouch young. Miller (in J. A. Allen, $1916 \mathrm{c}$, p. 589) reported from 5 to 8 pouch young, and Seba $(1734$, p. 57$)$ affirmed that there are 6 and sometimes 8 , or as many nipples as suckling young. These observations suggest that the prime mammary formula for Philander opossum is normally $3-1-3=7$. The same holds for Philander andersoni. Didelphis usually has 11 or 13 teats. Chironectes has 5, Lutreolina 9 or 11 . In pouchless didelphoids, the outer rows of teats may extend the length of abdomen and chest; the shorter median row is abdominal and has 1 to 5 teats. None are abdominal in the microbiotheriid Dromiciops.

\section{Cranial Characters and Comparisons (Figs. 10-12; Table 5)}

Skull elongate, mean condylobasal length about $67 \mathrm{~mm}$ in males, slightly shorter in females; dorsal surface nearly plane with little curvature between muzzle and braincase; occipital condyles turned slightly behind vertical plane of body of occipital bone; nasals long, posterior portions abruptly expanded at maxillofrontal suture then tapered to blunt points parted terminally, tips not extending anteriorly to vertical plane of first incisors; length of nasals more than $40 \%$ greatest 

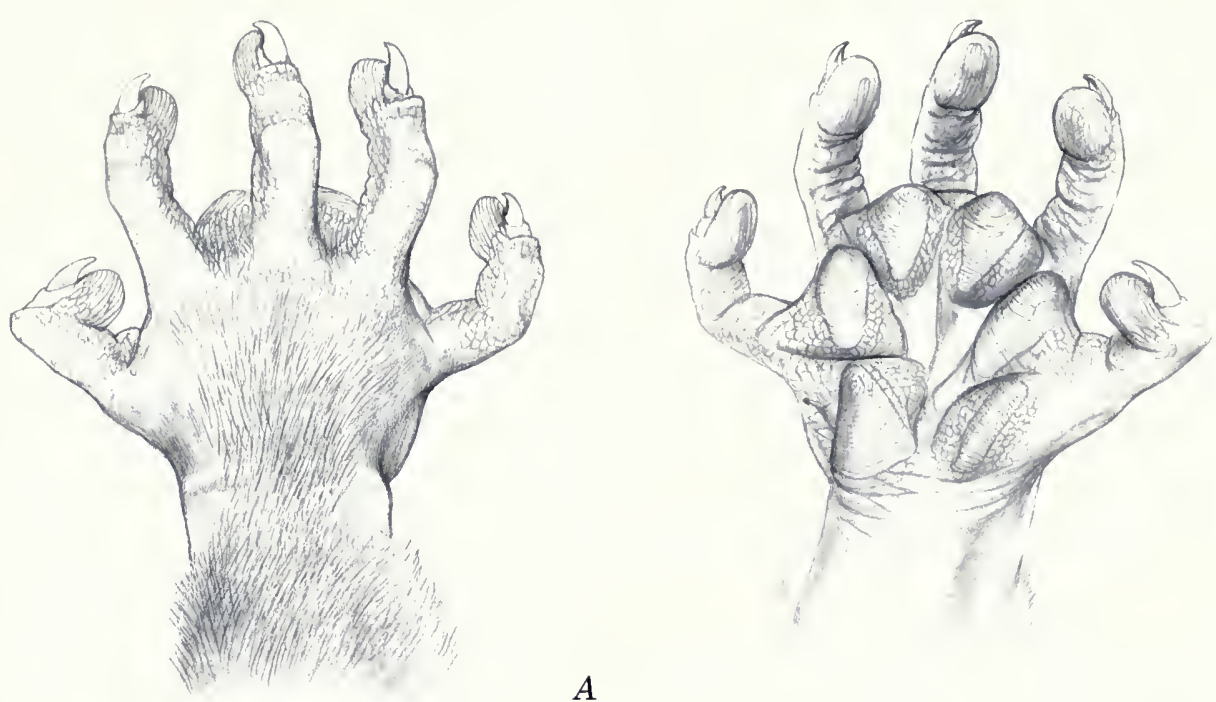

A

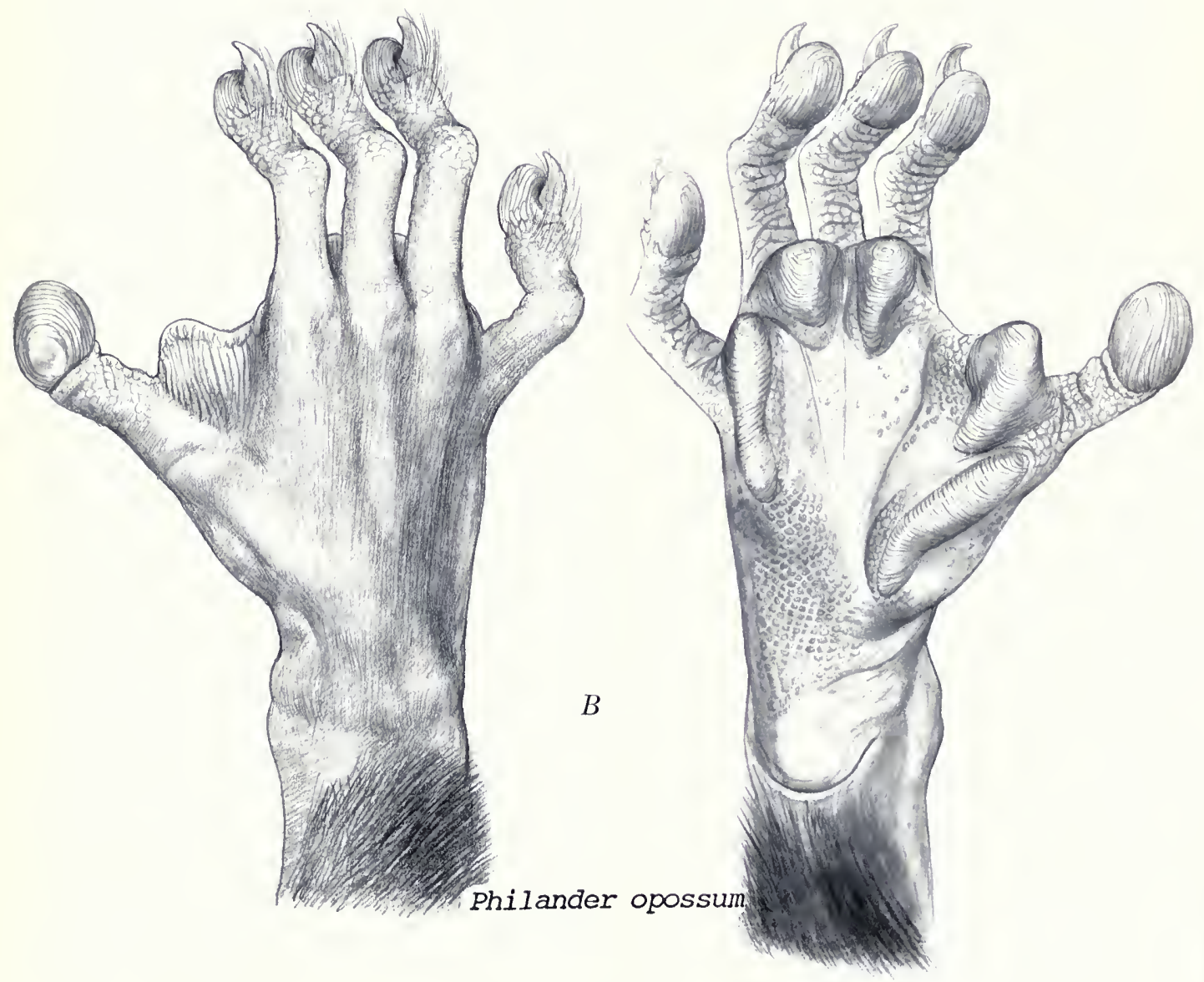


skull length and $60 \%$ midline length from tips of nasals to lambdoidal crest; combined tips of premaxillary bones rounded, extending less than 1 $\mathrm{mm}$ beyond base of $\mathrm{i}^{1}$.

Distance from lacrymal foramen to anterior edge of glenoid fossa less than zygomatic breadth; supraorbital borders square or rounded, not ridged or overhanging; postorbital constriction narrower than preorbital constriction; well-defined postorbital processes short, bluntly pointed; temporal ridges uniting behind to form a low sagittal crest connected with a well-developed nuchal or lambdoidal crest; shallow pit or depression at junction of temporal ridges; cranial crests developed early in both sexes.

Palatal vault not markedly arched, its contour nearly flat; palatal extension behind transverse plane of last molars equal to combined length of last 2 or $2 \frac{1}{2}$ molars; paired incisive foramina long, extending behind anterior plane of canines; paired maxillopalatal vacuities long, slitlike and extending from about the level of $\mathrm{m}^{1}$ to that of $\mathrm{m}^{3}$; paired posteropalatal vacuities usually present, short, ovate, extending from or behind plane of last molars to raised posterior palatal border; posterior choanae narrow, width measured across posterior base of pterygoid processes usually less than $10 \%$ of condylobasal length; tripartite auditory bulla, composed of tympanic wing of alisphenoid bone, separated from tympanic wing of petrous bone with ectotympanic bone suspended between, the components separate or touching but not fused (Hershkovitz, 1992a, Fig. 10, p. 25; Hershkovitz, in press).

Comparisons (Fig. 2)-Skull of Didelphis relatively broader, the frontal and maxillary sinuses more inflated, sagittal crest high, bladelike. Skull of Chironectes heavier, broader, braincase width about $35 \%$ of condylobasal length (less than $30 \%$ in Philander); angle between sagittal and frontal planes greater, nasals relatively shorter, less tapered behind, the terminal points spread as in $\mathrm{Lu}^{-}$ treolina; zygomatic breadth greater, palate wider, the posterior vacuities rectangular, posteromedian vacuities usually absent; occipital condyles not projecting behind vertical plane of supraoccipital bone, their combined width about half that of palate between molars; turbinate bones enlarged and produced behind to nearly posterior border of palatal vacuities. Lutreolina braincase relatively lon- ger than that of Philander, sagittal and nuchal crests heavier, postorbital region more elongate, braincase more deflected relative to palatal plane, frontal and maxillary sinuses more inflated, muzzle relatively shorter, nasals less than $38 \%$ greatest skull length and less than $55 \%$ midline length from nasal tips to lambdoidal crest.

In didelphoids generally, as growth continues after sexual maturity, crests enlarge and most cranial proportions shift.

\section{Dental Characters and Comparisons (Table 6, Figs. 13-20)}

The following dental formula for didelphids is the same for all living and known extinct didelphoids:

$$
\mathrm{i} \frac{1,2,3,4,5}{(1), 2,3,4,5}, \quad \mathrm{c} \frac{1}{1}, \quad \mathrm{p} \frac{1,2,3}{1,2,3}, \quad \mathrm{~m} \frac{1,2,3,4}{1,2,3,4}
$$

The same formula has persisted unchanged since no later than Early Cretaceous and likely Early Jurassic. Loss of $i_{1}$, shown in parentheses, and the staggered or crowded position of $i_{3}$ (Figs. 12, 13, 14, 16), evidence of Mesozoic mandibular contraction, are didelphoid hallmarks. The metacone of $\mathrm{m}^{1-3}$ is consistently larger than the paracone. The size relationship between metacone and paracone in eutherians varies within families and sometimes individually.

According to Luckett (1993), the deciduous or primary teeth of incisors and canines are nonfunctional and vestigial. The functional incisors and canines are successional or second generation. The functional premolars are mixed: $\mathrm{p} 1$ and $\mathrm{p} 2$ are the unreplaced deciduous teeth; p3, the last tooth to erupt, succeeds a functional, fully molarized deciduous p3. This tooth is also the first to erupt. Molars are unreplaced primary teeth (Fig. 14).

The sequence of dental eruption revealed by didelphids at hand and by studies of prenatal and early postnatal Didelphis virginiana by McCrady (1938) and Berkovitz (1978) indicated the following (teeth enclosed by parentheses erupt at about the same time):

dp3, p2, i(2-4), i5, il, (c, pl), m1, m2, m3, p3, m4 dp3, p2, i(2-4), i5, -, (c, pl), ml, m2, m3, p3, m4

FiG. 6. Cheiridia of Philander opossum. A, Right hand, dorsal and plantar aspects; B, right foot, dorsal and plantar aspects. 

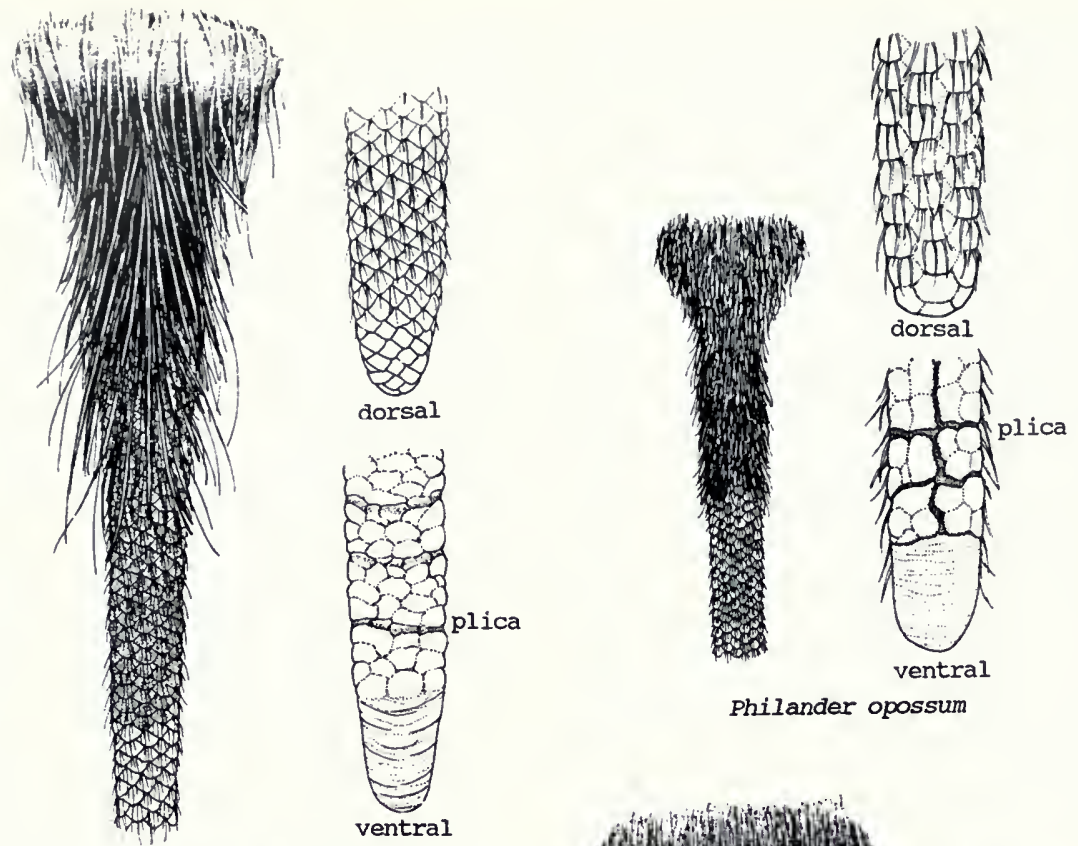

Didelphis marsupialis
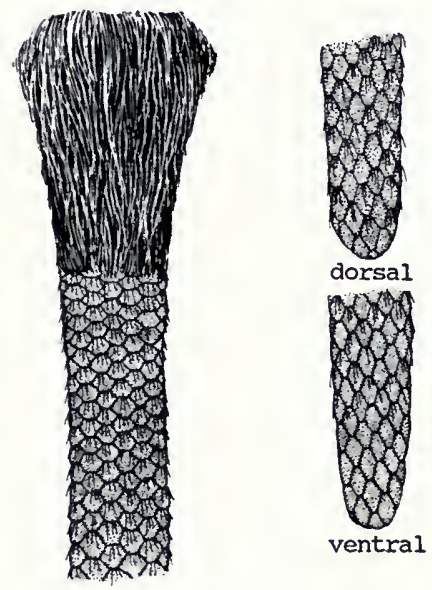

Chironectes minimus

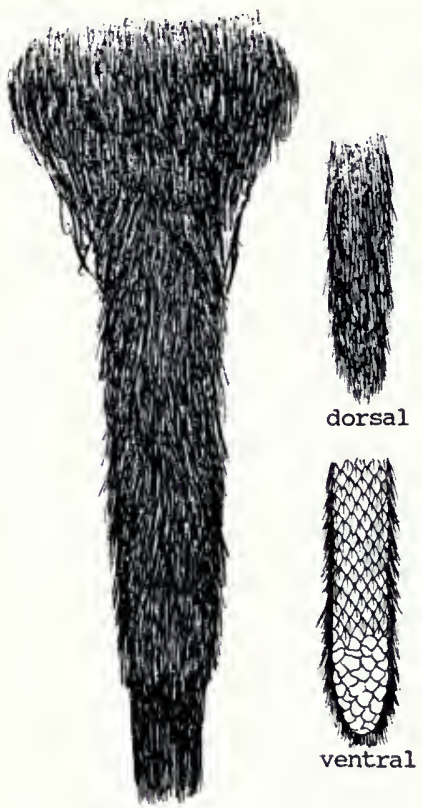

Lutreolina crassicaudata

Fig. 7. Basal (dorsal) and terminal (dorsal and ventral) caudal segments of Didelphis, Philander, Chironectes, and Lutreolina. Plicas are cutaneous folds of prehensile tails.

\section{Descriptions of Individual}

Teeth of Philander

UPPER INCISORS (Fig. 15)-Five in number, first (i $\left.{ }^{1}\right)$ usually largest, sometimes as small as any of $\mathrm{i}^{2-5} ; \mathrm{i}^{1}$ most distinctive of series, subcylindroform, orthodont or slightly prodont, the opposing pair convergent for about two-thirds their length, their pointed tips often divergent; unworn $\mathrm{i}^{2-5}$ uniformly premolariform (tridentate), crowns slightly to broadly overlapping except in old, worn teeth; $i^{2}$ from half to as high as $i^{1}$, slightly less than that 


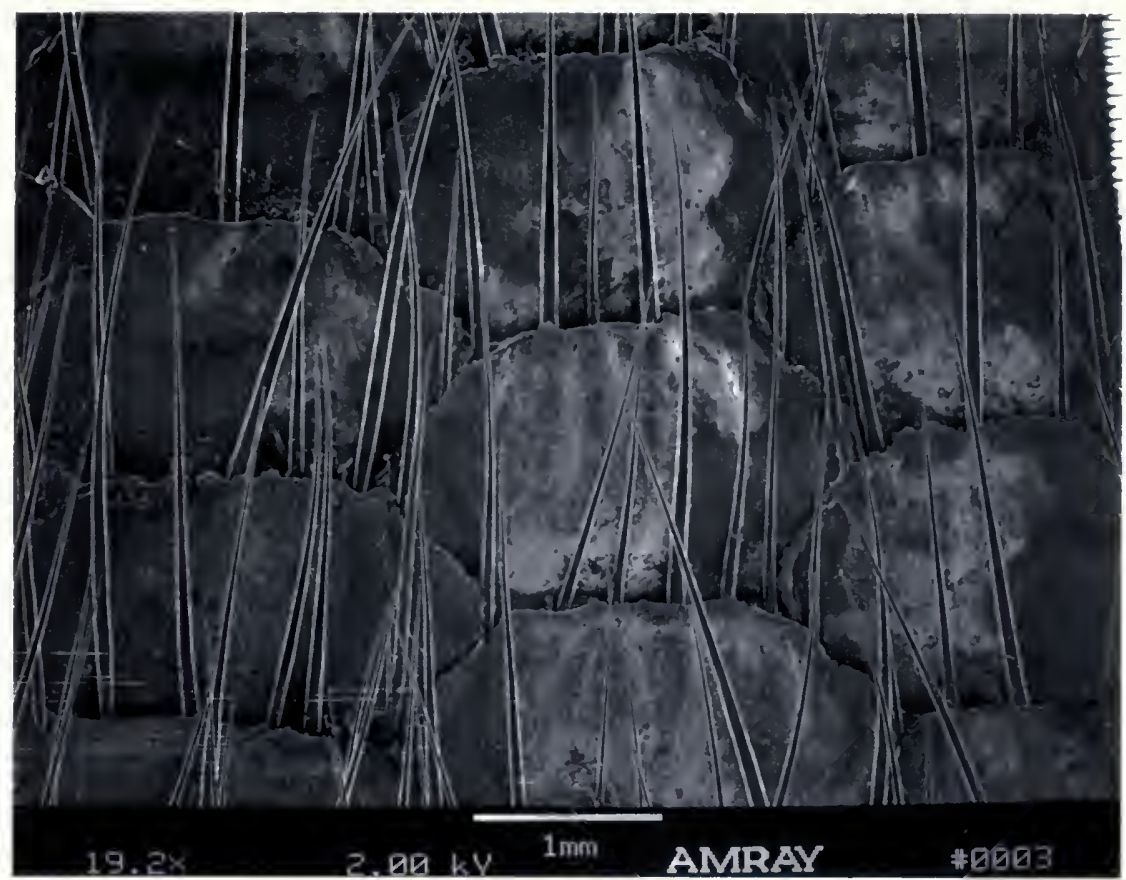

FIG. 8. Section of Philander opossum tail, ventral surface, showing spiral arrangement of scales and interscutular bristles, usually five per scale.

of $\mathrm{i}^{3,4}$; a small diastema present between $\mathrm{i}^{1}$ and $\mathrm{i}^{2}$; mesiostyle $(a$ or $A)$ of $\mathrm{i}^{2-5}$ weak, distostyle ( $b$ or $E)$ less defined or obsolete.

LOWER INCISORS-Four in number (2-5), the phylogenetic first lost; all uniform in structure but decreasing in size from numerical first $\left(i_{2}\right)$ to last $\left(i_{5}\right)$; all incisors markedly recumbent seen from lingual aspect; crowns usually well separated in adults but sometimes touching in young; each tooth with labial surface more or less spatulate, crown ovate, root cylindrical, tapered; lingual surface smooth, the median ridge or torus broadly sloping; occlusal groove often present on lingual surface; terminal styles obsolete; numerical sec- ond (phylogenetic $i_{3}$ ) staggered between $i_{2}$ and $i_{4}$ as in all didelphoids (Figs. 13, 14, 16).

REMARKS-Marsupial dental evolution appears to have been most manifest in the incisor field. The staggered $i_{3}$ marks divergence of the Didelphimorphia from the basic metatherian stock. Only microbiotheres, including Pucadelphys andinus Marshall and de Muizon, 1988 (Marshall et al., 1995), of the Early Paleocene of Bolivia are the known members of the unstaggered-incisortoothed Microbiotheriomorphia (cf. Hershkovitz, 1992; 1995).

UPPER CANINE-Long, slender, smooth, recurved with axis directed slightly forward, its

TABLE 3. Vertebral count of largest American marsupials. Number of transitional vertebrae also included in count of caudal vertebrae as customary. Number of cervical vertebrae is consistently 7 , of sacral, 2 , in all marsupial taxa examined. Sample number in parentheses.

\begin{tabular}{lllll}
\hline \multicolumn{1}{c}{ Taxon } & Thoracic & Lumbar & \multicolumn{1}{c}{ Caudal } & Transitional \\
\hline Philander opossum & $13(5)$ & $6(2) ; 7(3)$ & $27 ; 28$ & $4 ; 5(3)$ \\
Philander andersoni & $13(2)$ & $6(2)$ & $27 ; 31$ & $4 ; 5$ \\
Didelphis marsupialis & $13(4)$ & $6(2) ; 7(2)$ & $31 ; 32(2)$ & $5(3)$ \\
Didelphis albiventris & $12(2) ; 13(2)$ & $5 ; 6 ; 7$ & $30(2) ; 31$ & $4 ; 5 ; 6$ \\
Chironectes minimus & $13(7) ; 14$ & $6(8)$ & $27(2) ; 28(2) ; 29$ & $4(5) ; 5(2)$ \\
Lutreolina crassicaudata & $13(2)$ & $6 ; 8$ & $11+(2)$ & $4 ; 5$ \\
\hline
\end{tabular}




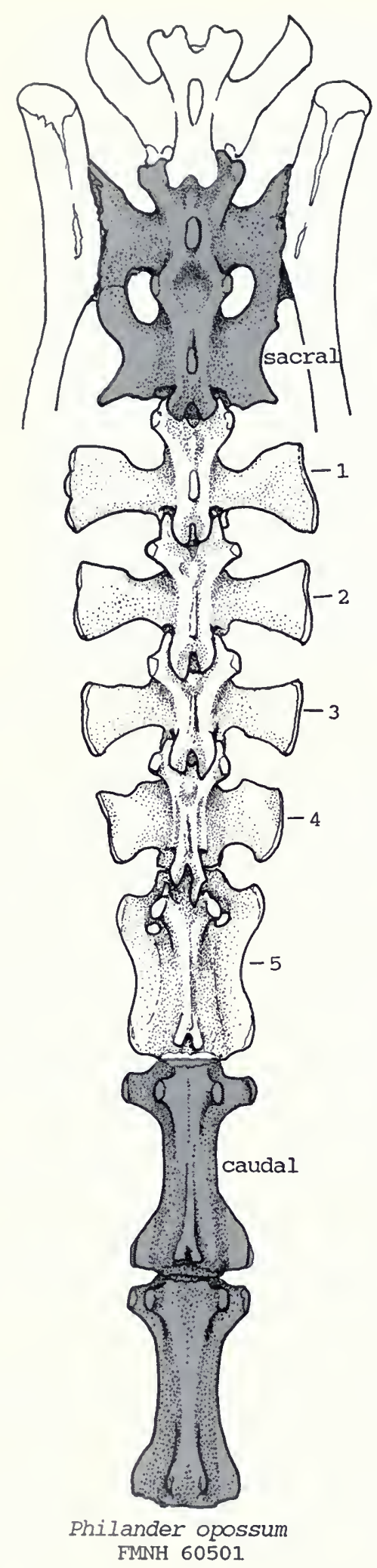

growth continuous past sexual maturity; cingulum and accessory conules absent; diastema between canine and last incisor approximately equal to or less than greatest diameter of canine.

Lower CANINE-Shorter, weaker than upper, more or less recurved, its bulk about half that of upper; well differentiated from incisors, about twice their height, without diastema.

UPPER PREMOlars - Teeth two-rooted, unicuspidate with well-developed ledgelike lingual and buccal cingula; first premolar about half or less the bulk of second, the diastema between approximately equal to crown length in old adults, about one-half to one-third crown length in young adults; middle premolar larger than last, often equal, infrequently smaller; unworn first premolar with terminal styles usually present and well defined; middle premolar with terminal styles poorly defined or absent except style $b$ or $E$, the mesiostyle (parastyle, $a$ or $A$ ) less frequently present than the distostyle ( $b$ or $E$ ); eruption of replacement $\mathrm{p}^{3}$ precedes that of $\mathrm{m}^{4}$, styles poorly differentiated or absent; FMNH 114685 with an incipient but well-defined metacone.

Upper Deciduous Third Premolar (the only upper deciduous tooth) (Fig. 17)-Size about equal to that of $\mathrm{m}^{4}$, general outline and morphology as in $\mathrm{m}^{1}$, conules $A, B$ often well developed, conule $C$ larger than $B, D$ greatly reduced or absent, $E$ indicated.

Lower Premolars - Teeth two-rooted, unicuspidate with ledgelike lingual and buccal cingulids poorly defined, talonid basin simple; lower first premolar similar in size and shape to upper first and likewise separated by diastema from middle premolar, distostylid ( $b$ or $E$ ) more or less defined, mesiostylid ( $a$ or $A$ ) obsolete or absent; middle premolar as high or higher than incisors and nearly as high as canine, distostylid present, mesiostylid minuscule; last premolar like middle but smaller, buccal cingulum usually poorly defined, talonid basin slightly more developed; permanent $\mathrm{p}_{3}$ erupts before $\mathrm{m}_{4}$.

Lower Deciduous Third Premolar (the only lower deciduous tooth) (Fig. 17) -Like first per-

FiG. 9. Transitional caudal vertebrae 1-5 (untinted) of Philander opossum (FMNH 60501) between tinted sacral and first typical caudal (coccyxial) vertebrae. Tran sitional vertebra 5 differs notably from both the caudal behind and transitional 4 ahead. 
Table 4. Nipple formulae of the largest didelphoids. Number of samples more than one shown in parentheses; specific names are those of cited source; formulae from Hershkovitz field notes are of captured animals, the prepared specimens preserved in FMNH or USNM; other preserved specimens in the museum collection are shown as FMNH.

\begin{tabular}{|c|c|c|}
\hline Taxon & $\begin{array}{c}\text { Prime Nipple Formulae } \\
\text { [Functional formulae } \\
\text { in brackets] }\end{array}$ & Source \\
\hline \multicolumn{3}{|l|}{ DIDELPHIDAE } \\
\hline \multicolumn{3}{|l|}{ Didelphini } \\
\hline \multicolumn{3}{|l|}{ Philander } \\
\hline andersoni & {$[2-1-2]$} & da Silva and Patton, pers. comm. \\
\hline andersoni & {$[2-0-2]$} & da Silva and Patton, pers. comm. \\
\hline andersoni & {$[3-0-3]$} & da Silva and Patton, pers. comm. \\
\hline andersoni & $3-1-3=7(4)$ & da Silva and Patton, pers. comm. \\
\hline opossum & $3-1-3=7(4)$ & Thomas, 1888 \\
\hline opossum & 7 & Osgood, 1921:75 \\
\hline opossum & $3-1-3=7(4)$ & Hershkovitz, field notes (FMNH) \\
\hline opossum & $3-1-3=7(4)$ & da Silva and Patton, pers. comm. \\
\hline \multicolumn{3}{|l|}{ Didelphis } \\
\hline albiventris & $5-1-5=11(2)$ & Hershkovitz, field notes (USNM) \\
\hline albiventris & {$[4-1-3=8](2)$} & Hershkovitz, field notes (USNM) \\
\hline albiventris & $11(2)$ & Carlsson, 1903:490 \\
\hline albiventris & $5-1-5=11$ & Krieg, 1924:652, Fig. I \\
\hline marsupialis & $5-1-5=11(4)$ & Hershkovitz, field notes (USNM) \\
\hline marsupialis & {$[4-1-4=9](3)$} & Hershkovitz, field notes (USNM) \\
\hline marsupialis & {$[10]$} & O'Connell, 1979:81 \\
\hline virginiana & 13 & Hartman, 1920:255 \\
\hline virginiana & 13 & McCrady, 1938:183 \\
\hline \multicolumn{3}{|l|}{ Lutreolininae } \\
\hline \multicolumn{3}{|l|}{ Lutreolina } \\
\hline crassicaudata & $4-1-4=9$ & Thomas, 1888:335 \\
\hline crassicaudata & $5-1-5=11$ & Krieg, 1924:652, Fig. c \\
\hline crassicaudata & 9 & Osgood, 1921:75 \\
\hline crassicaudata & {$[7]-9$} & Creighton, 1984:24 \\
\hline \multicolumn{3}{|l|}{ Chironectinae } \\
\hline \multicolumn{3}{|l|}{ Chironectes } \\
\hline minimus & $2-1-2=5$ & Hershkovitz, field notes (USNM) \\
\hline minimus & 5 & Enders, 1966 \\
\hline minimus & [4] 5 & Krieg, 1921 \\
\hline minimus & 4 to 5 & Mondolfi and Medira Padilla, 1958:155 \\
\hline
\end{tabular}

manent molar $\left(m_{1}\right)$ except one-fourth to one-third smaller.

UPPER MOLARS $\left(\mathrm{m}^{1-4}\right)$ (Figs. 17-20)-Crowns wider than long; second molar $\left(\mathrm{m}^{2}\right)$ longer and larger than first $\left(\mathrm{m}^{1}\right)$, slightly shorter to longer than third $\left(\mathrm{m}^{3}\right)$, fourth molar $\left(\mathrm{m}^{4}\right)$ about half bulk of third; mesiostyle and distostyle present in $\mathrm{m}^{1-4}$, but distostyle may be obsolete or absent in $\mathrm{m}^{4}$; trigon cusps of $\mathrm{m}^{1-3}$ with metacone largest, paracone smallest, of $\mathrm{m}^{4}$ paracone largest, metacone smallest, often minute; stylocone (conule $B$ ) always large and prominent in $\mathrm{m}^{1-3}$; style (conule $C)$ as large or larger than stylocone in $\mathrm{m}^{\prime}$, subequal or smaller in $\mathrm{m}^{2}$, subequal or usually smaller and often rudimentary or merely indicated in $\mathrm{m}^{3}$; in $\mathrm{m}^{4}$ buccal cusps small or rudimentary; accessory conules derived from buccal conules $B$,
$D$, from well defined to mere crenulations, or absent; crest of $\mathrm{m}^{3}$ metacrista longest of molar series; paracrista connected with conule $B$.

LOWER MOLARS $\left(m_{1-4}\right)$-Lingual cingulids and neocingulids absent; second or third molar largest, fourth smallest, largest cusp (paraconid or protoconid, 1) progressively larger than metaconid from $m_{1}$ to $m_{4}$; paraconid smaller than or subequal to metaconid, hypoconid larger than entoconid; mesiostylid ( $a$ or $A$ ) not certainly definable in every tooth, distostylid ( $b$ or $E$ ) well defined, its size increasing from first to last tooth, in $\mathrm{m}_{4}$ often as high or higher than paraconid; postentoconid larger than distostylid, becoming larger than paraconid in following teeth.

VARIATION-Consistent dental differences between Philander opossum and Philander ander- 

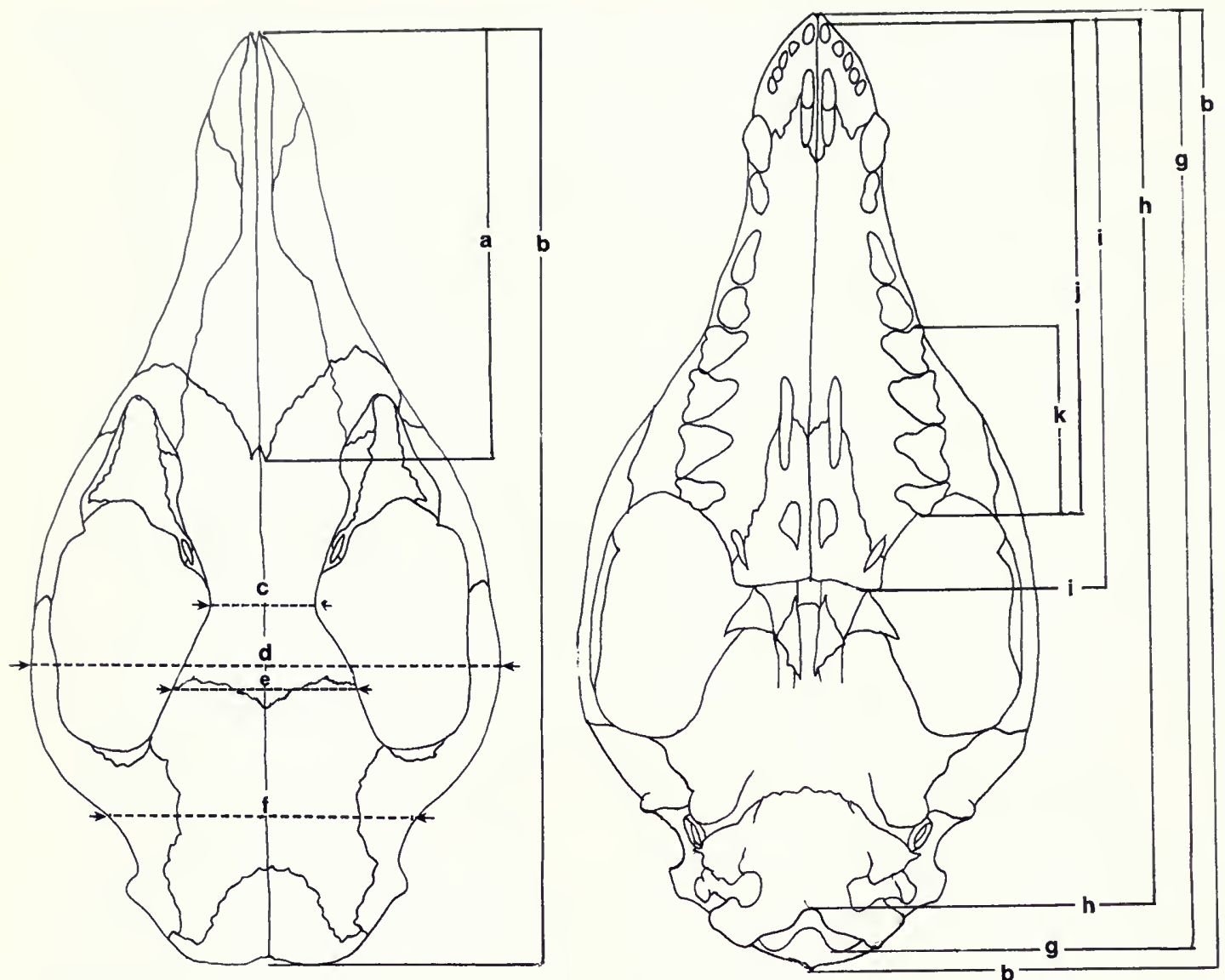

FIG. 10. Philander opossum, dorsal and ventral aspects, showing points of cranial measurements. a, nasals; b, greatest length of skull; c, least interorbital breadth or postorbital constriction; d, zygomatic breadth; e, postorbital width; f, braincase, greatest width; g, condylobasal length; h, basal length; i, palatal length; j, length upper tooth row $\left(\mathrm{i}^{1}-\mathrm{m}^{4}\right) ; \mathrm{k}$, length upper molar row $\left(\mathrm{m}^{1-4}\right)$.

soni have not been observed, but geographic differentiation does occur. In a comparison of "Philander mcilhennyi" from Balta, Perú, with sympatric Philander opossum quica, Gardner and Patton (1972, p. 3) noted a relatively deep indentation of the labial margin of $\mathrm{m}^{3}$ present in the first species but not in the second. The indentation, or so-called "notch," is the angle or valley between conule $C$ (stylar cone $C$ ) and adjacent conule $B$ (Fig. 19).

In four specimens at hand of Balta Philander andersoni mcilhennyi, conule $C$ of $\mathrm{m}^{3}$ is well developed or molarized in three females but quite worn in the single male. In 8 adults of sympatric Balta $P$. opossum quica, I find conule $C$ distinctly less developed in three females and rudimentary or virtually absent in 5 males. Examination of all available specimens from throughout the range of the genus, however, reveals that variation from present to absent, or degree of molarization of conule $C$, is about the same for both species (Table 7).

Conule $C$, like any other derived excrescence of the buccal shelf, is firmly established, cusplike, and usually subequal to conule $B$ in $\mathrm{m}^{1}$ and $\mathrm{m}^{2}$. In $\mathrm{m}^{3}$, however, it varies geographically from a rudiment or anlage to a fair-sized cusp nearly equal in bulk to conule $B$ (Fig. 19). Its molarization increases gradually from the most primitive and geographically central forms of $P$. andersoni and $P$. opossum to their most derived or geographically peripheral representatives.

Comparisons-Dentition of Didelphis is essentially like that of Philander. That of Chironectes is generally heavier, average transverse width of $\mathrm{m}^{3}$ about $6 \mathrm{~mm}$ (4.5 in Philander), arcade of up- 

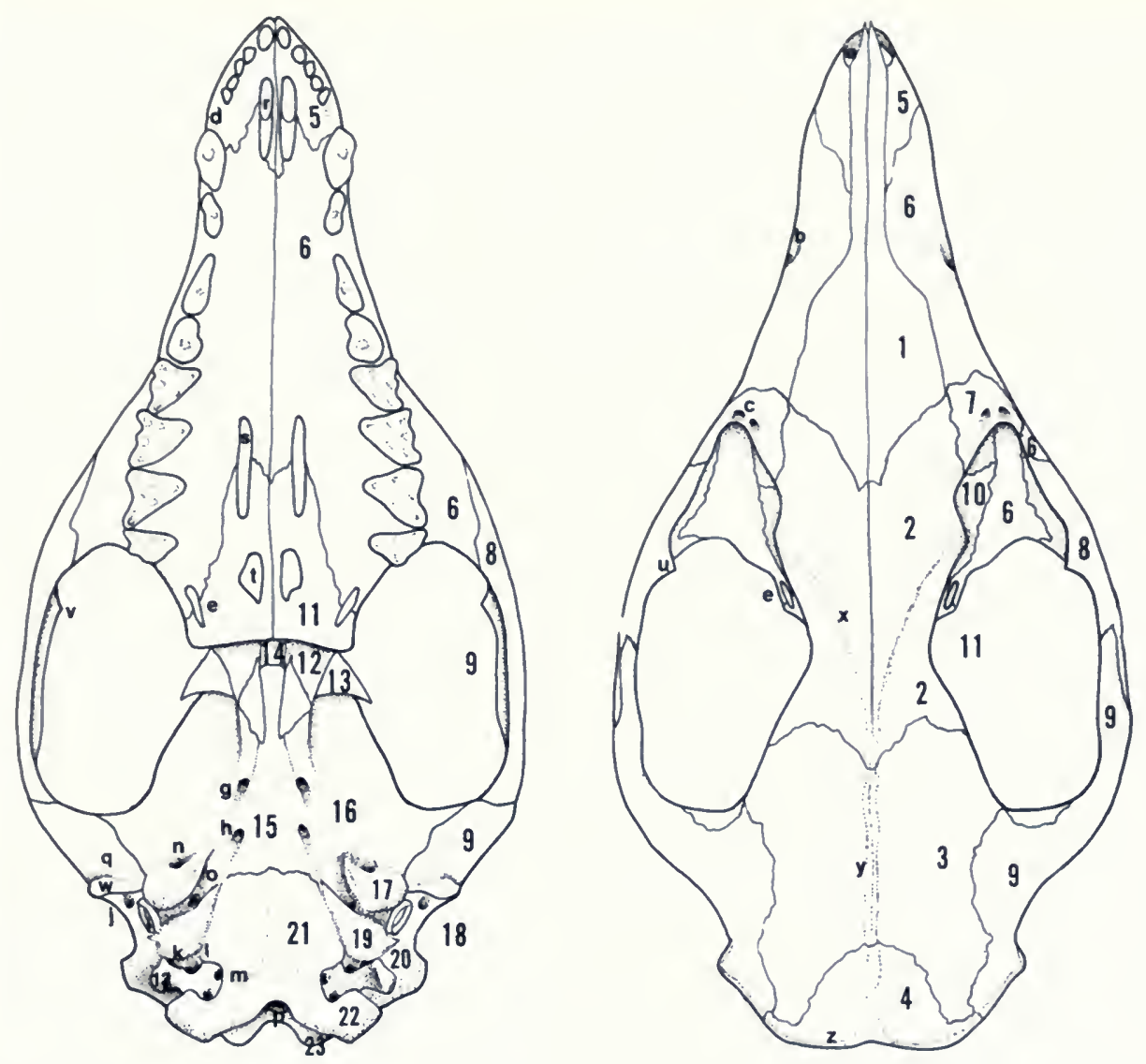

FiG. 11. Diagram of didelphid skull, ventral and dorsal aspects, showing topographic features. See Table 5 for explanation of symbols.

per and lower incisors more broadly curved with second lower $\left(i_{3}\right)$ less abruptly staggered; unworn incisors of Chironectes triangular in lateral outline, but shape more nearly equilateral than obtuse; upper incisors of Chironectes and Lutreolina notably inflected inward and upward; first premolars less reduced, gap between first and middle premolars narrower in Chironectes, varying from less than half the crown length of anterior tooth to absent; molar crowns more hypsodont in Chironectes and Lutreolina, outline of occlusal surface more nearly square or rectangular than triangular, the outer posterior angle, particularly of $\mathrm{m}^{3}$, less produced.

\section{Dental Abnormalities}

\section{Supernumeraries}

Philander andersoni andersoni
AMNH 72017, adult \&, Río Curaray, Loreto, Perú

Left supernumerary $\mathrm{m}^{\prime}$, slightly smaller than normal $\mathrm{m}^{\prime}$, rotated $180^{\circ}$ with buccal shelf lingual.

\section{Philander opossum fuscogriseus}

AMNH 34373, adult ơ, Bagadó, Chocó, Colombia

Left supernumerary $\mathrm{m}^{4}$, fully erupted, slightly smaller than normal $\mathrm{m}^{4}$, otherwise similar.

Right supernumerary $\mathrm{m}^{4}$ nearly fully erupted but impacted and rotated about $90^{\circ}$ with buccal side anterior and overlapped by posterior portion of normal $\mathrm{m}^{4}$. Lower molars normal in number and form.

\section{Philander opossum quica}

LSumz 12009, adult + , Balta, Ucayali, Perú Right supernumerary pm', in diastema be- 
TABLE 5. Explanation of cranial symbols $1-23$ and $\mathrm{a}-\mathrm{z}$ in Figures 11 and $12 \mathrm{~A}$.

Bones

1. Nasal

2. Frontal

3. Parietal

4. Supraoccipital

5. Premaxillary

6. Maxillary

7. Lacrymal

8. Jugal, zygomatic

9. Squamosal, temporal

10. Sphenoid (includes orbitosphenoid, alisphenoid, presphenoid, basisphenoid, pterygoid)

11. Palatine

12. Orbitosphenoid

13. Pterygoid process

14. Presphenoid

15. Basisphenoid

16. Alisphenoid

17. Alisphenoidal wing of auditory bulla

18. Ectotympanic

19. Periotic wing of auditory bulla

20. Mastoid (temporal)

21. Basioccipital

22. Occipital condyle

23. Exoccipital

\section{Foramina, Fissures, Processes, Fossae, and Crests}

a. External nares

b. Infraorbital foramen

c. Lacrymal foramina or canals

d. Canine fossa

e. Posterolateral vacuity or foramen

f. Sphenorbital fissure (concealed above 11)

g. Foramen rotundum

h. Foramen ovale

i. Tympanic membrane and auditory meatus (see 18)

j. Postglenoid foramen

k. Stylomastoid foramen

1. Jugular foramen

m. Hypoglossal foramen and or condylar foramen

n. Carotid foramen or canal

o. Anterior lacerate foramen or petrotympanic fissure

p. Foramen magnum

q. Glenoid fossa

r. Premaxillary or incisive foramen

s. Maxillopalatine or mesolateral vacuity

t. Posteromedial or palatine vacuity

u. Ascending postorbital (zygomatic) process

v. Ascending postorbital (zygomatic) process

w. Postglenoid process

$x$. Temporal ridge

y. Sagittal crest

z. Lambdoidal crest

tween normal first and second premolars, fully erupted, normal in appearance except rotated about $100^{\circ}$ with posterior border linguad and slightly anteriad; heel with pronounced distostylid (hypoconulid) as in left $\mathrm{pm}^{\mathrm{I}}$. "Normal" first premolar without talonid presumably a result of crowding by developing supernumerary.

FMNH 114702, adult ơ, San Ramón, El Beni, Bolivia

Right supernumerary $\mathrm{i}_{5}$, behind normal $\mathrm{i}_{5}$, occludes with normal $i^{5}$; normal $i_{5}$ occludes with $\mathrm{i}^{4}$ and so on to $\mathrm{i}_{2}$, which bites into diastema between $\mathrm{i}^{1}$ and $\mathrm{i}_{2}$; position of $i_{1}$ more medial and anteriad compared to left homologue; jaw, teeth, and occlusion otherwise normal.

FMNH 114685, ?

Left upper premolar with incipient but well-defined metacone.

\section{Missing molar \\ Philander opossum opossum}

USNM 393600, adult $q$, Utinga, Pará, Brasil $\mathrm{m}^{4}$ missing; no sign of alveolus but arcade otherwise normal; $\mathrm{m}_{4}$ normal; teeth moderately worn.

\section{Sexual Dimorphism}

Mature males with four molars fully erupted average larger than comparable females in body mass, skull length, and canine size. Postcanine teeth also average larger, but relative to skull length no larger than those of females or even smaller; the braincase is relatively narrower in males, cranial crests more developed, but the sagittal crest of old males is sometimes equaled by that of extremely large or old females.

\section{Karyology}

Descriptions of karyotypes of the present and supposed species of Didelphidae (Table 8) were first published in nearly 20 papers. All publications were critically reviewed, with new data added by Reig et al. (1977). The authors studied and described the chromosomes of 177 species of American marsupials representing nine generc and 22 species, including those considered in this report (Table 8). To avoid repetition and extralimital discussion, only Reig et al. (1977) is citer here for documentation.

The "standard" karyotype as understood b! Reig et al. (1977) is present in all treated as di delphids with few minor exceptions. All listed is Table 8 share the diploid number of 22 . The fun damental number is 20 , except in $D$. virginiana. 

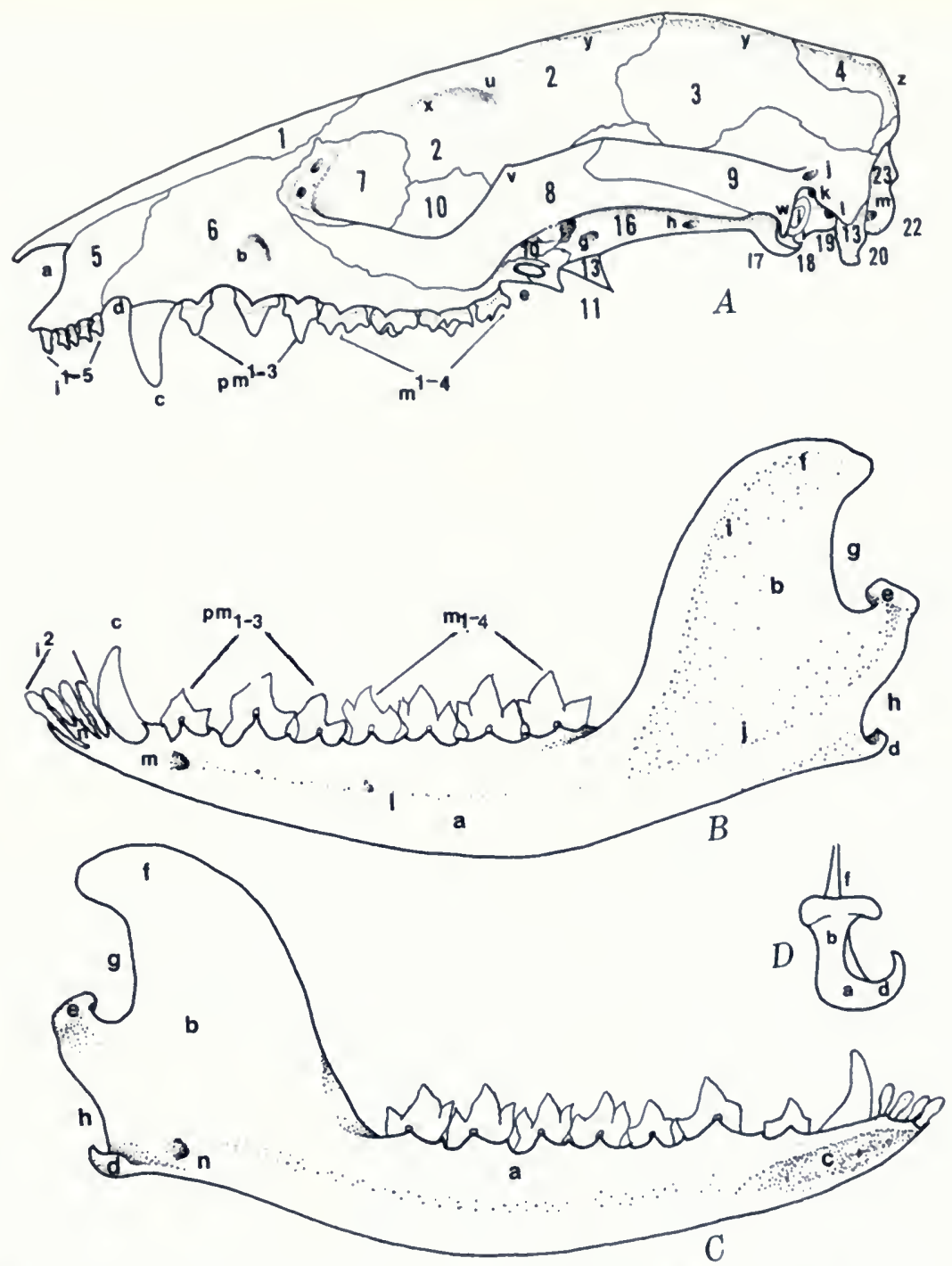

FiG. 12. A, Diagram of left side of Philander opossum skull; B, left mandible, buccal side; C, lingual side; D, posterior view. See Table 5 for names of cranial features and below for those of buceal and lingual sides of mandible: $a$, horizontal ramus; $b$, ascending ramus and masseteric fossa; $c$, symphysis; d, angular process; e, condyloid process; $f$, coronoid process: g, superior noteh; $h$, inferior or lunate notch; $i$, stiperior masseteric line; $j$, inferior masseteric line; $k$. mylohyoid line (not shown); I, horizontal masseteric line; $m$. mental foramen; $n$, mandibular foramen.

where it is 32 . Sex chromosomes are acrocentric in all but are metacentric in the $\mathrm{X}$ chromosome of $D$. virginiana and $L$. crassicaudata. It appears that the most variable complement among the four genera of Table 8 is $D$. virginiana.

\section{Parasites}

Captured marsupials are mostly parasitized by fleas, lice, and staphylinid beetles. These mobile parasites usually leave a live caged animal shortly after capture and desert the host almost immediately at death.

The list of parasites is not exhaustive. It was compiled mainly as a source of reference for discussions on marsupial zoogeography. The taxonomic names used are those of the sources cited.

Viruses

Mucambo (Potkay, 1977; Pará, Brasil; Shope, 1967b (in Potkay, 1977, q.v.)) 


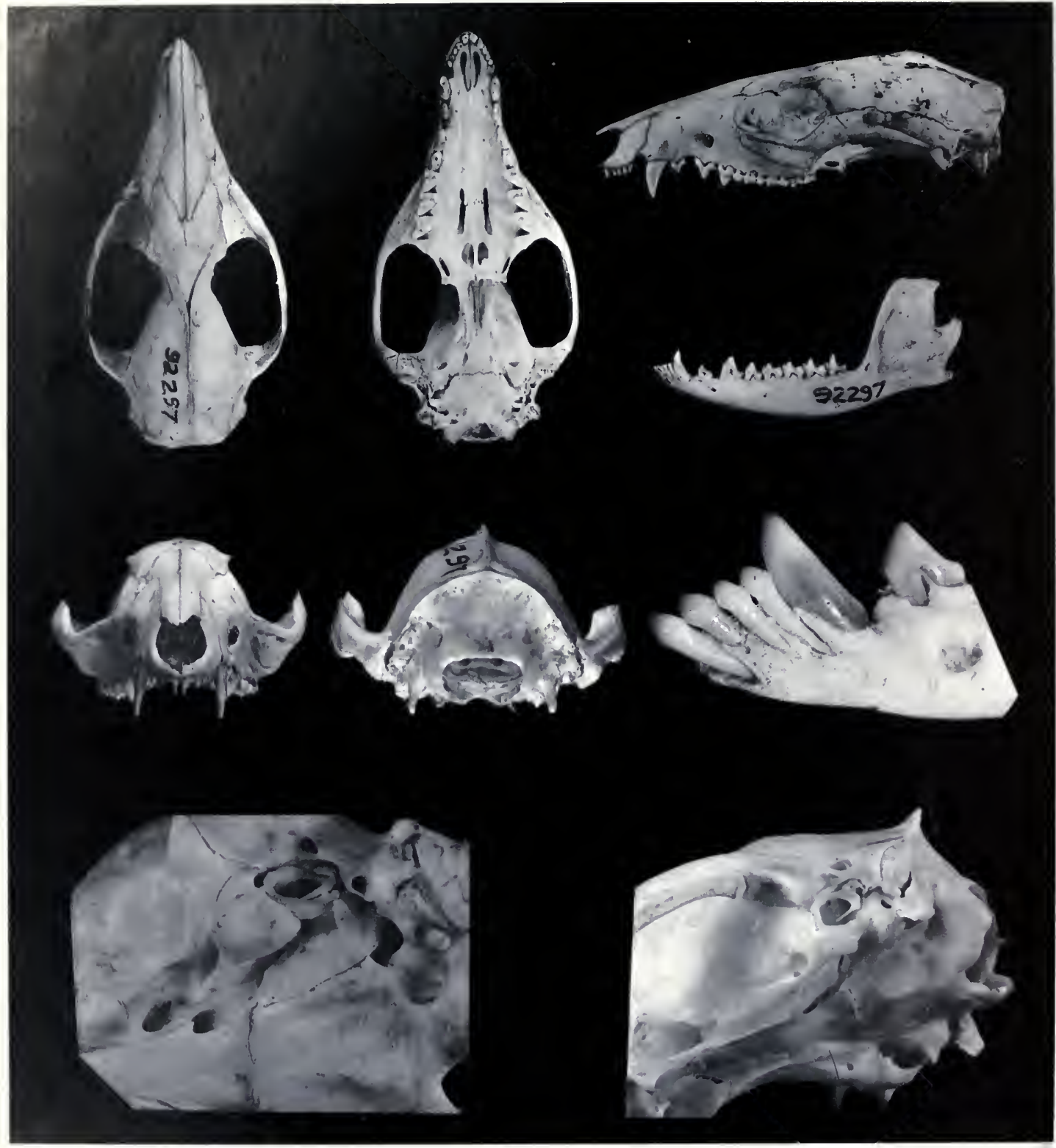

FIG. 13. Skull of Philander opossum. Upper row, dorsal, ventral, lateral aspects; middle row, anterior, posterior aspects and left lateral $i_{2}-\mathrm{pm}_{1}$ (note staggered $i_{3}$ with buttress); bottom row, two posterior portions of basicranium (see Figs. 11, 12 and Table 5 for identification of parts).

Venezuelan equine encephalitis (Almirante, Panamá; Grayson \& Galindo, 1968, in Potkay, 1977)

Eastern equine encephalitis (Bahia, Brasil; Shope et al., 1966, in Potkay, 1977)

St. Louis encephalitis (Bahia, Brasil; Shope et al., 1966)
Group B (mosquito borne)

Ilheus (Bahia, Brasil; Laemmert, 1967)

Yellow fever (Bahia, Brasil; Laemmert et al., 1946)

\section{Group C}

Itaqui (Bahia, Brasil; Shope, 1967a) 

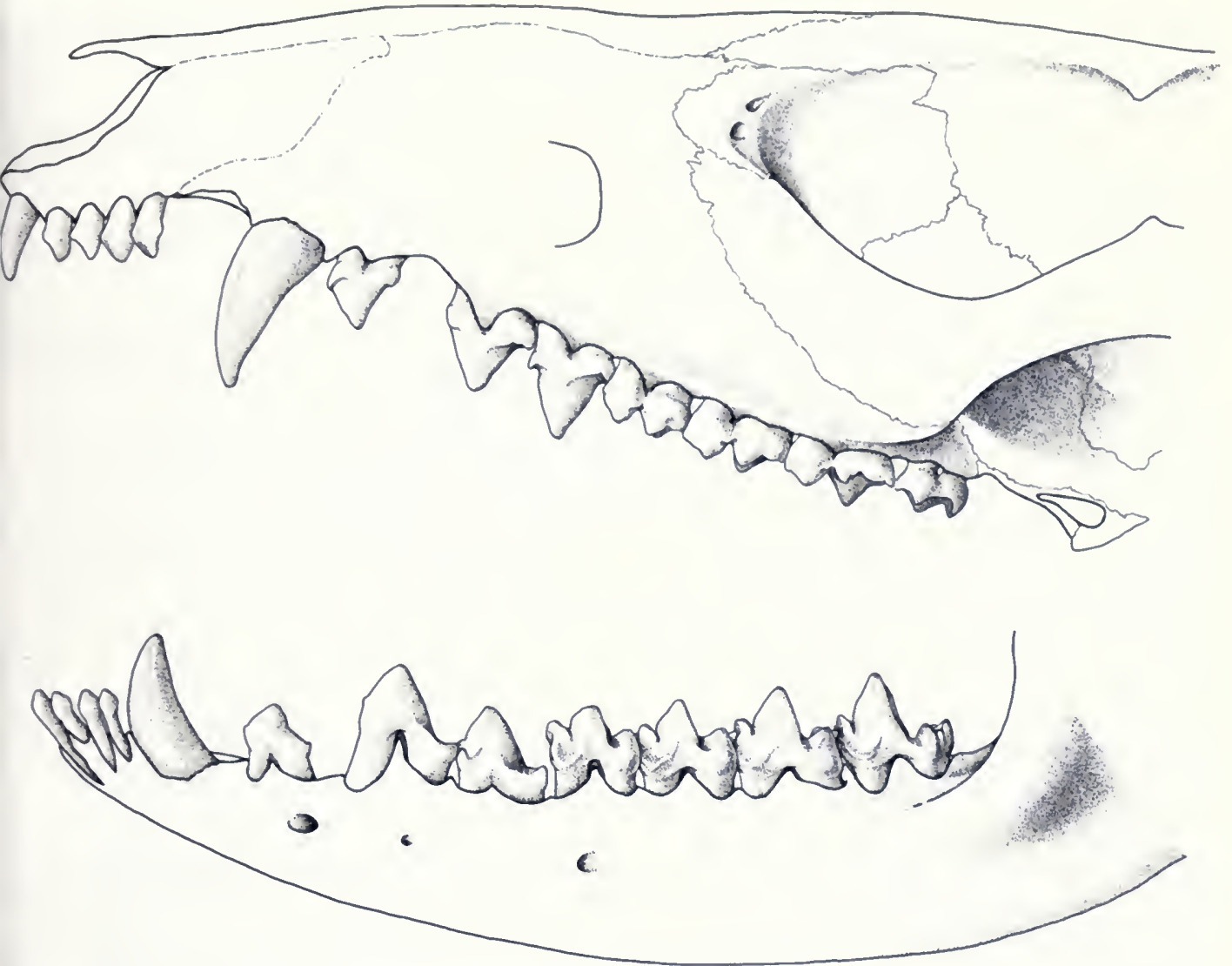

Fig. 14. Upper and lower jaws with tooth rows of Philander opossum.

Capim Group

Acara (Bahia, Brasil; Belém Virus Laboratory, 1976c)

Mosquito-borne group

Turlock (Bahia, Brasil; Shope et al., 1966)

Phlebotomus-borne

Itaporanga (Bahia, Brasil; Trapp \& Shope, 1967)

Vescicular stomatitis group

New Jersey strain (Panamá; Tesh et al., 1969)

Indiana strain (Panamá; Tesh et al., 1969)

Unknown vectors

Piry (Bahia, Brasil; Belém Virus Laboratory, 1967a)

Pacui (Bahia, Brasil; Belém Virus Laboratory, 1967b)

Oochoristica braziliensis (Colombia; Baer, 1927)
Sparganum reptans (Imperial Bureau of $\mathrm{Ag}$ ricultural Parasitology, 1933)

Protozoa

Babesia brasiliensis Regendanz and Kikuth (Brasil; Regendanz \& Kikuth, 1928, p. 1567)

Sarcocystis garnhami (British Honduras; Lainson \& Shaw, 1969)

Trypanosoma cruzi (Brasil; Wood \& Wood, 1941; Costa Rica; Deane, 1961, 1964)

Trypanosoma rangeli-like (Pará, Brasil; Deane, 1964)

Fungus

Histoplasma capsulatum (Canal Zone, Panamá; Taylor \& Shacklette, 1962)

Mallophaga (lice)

Gliricola porcelli (São Paulo, Brasil; Hopkins, 1949) 


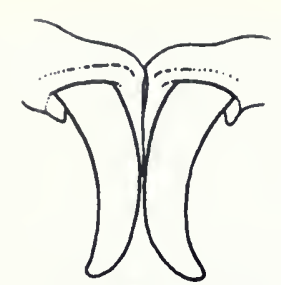

Philander opossum FMNA 16398

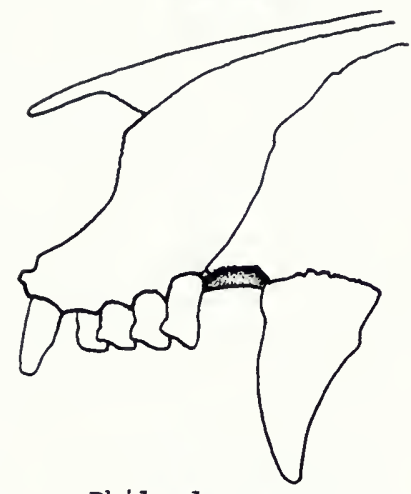

philander opossum FMNH 114698

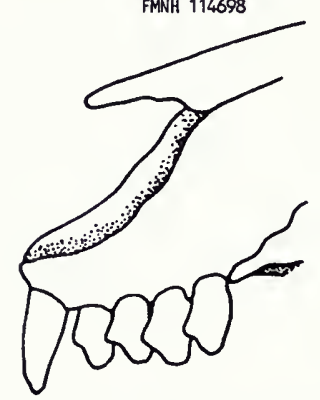

Philander opossum FMNH 114711
$\boldsymbol{A}$

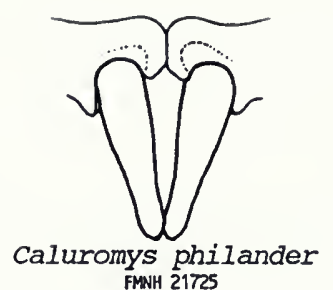

$\boldsymbol{B}$

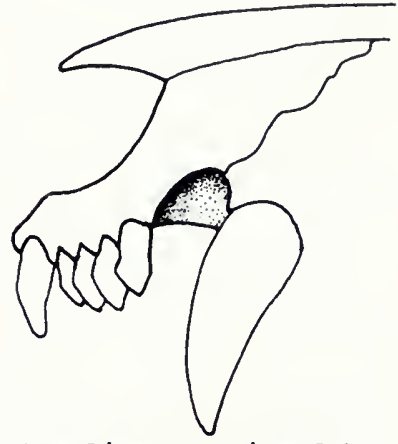

Lutreolina crassicaudata FNN 53944

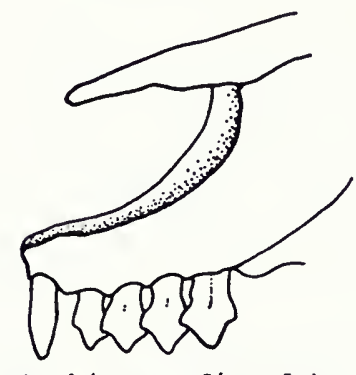

Metachirus nudicaudatus FMNH $207 \%$

Fig. 15. Upper incisors and canine of Philander opossum compared with outgroups. A, P. opossum (FMnH 16398), $\mathrm{i}^{1-1}$ variably convergent proximally, divergent distally; Caluromys philander (FMNH 21725 ), $\mathrm{i}^{1-1}$ convergent to tip. B, P. opossum (FMNH 114698), premaxillary fossa for lower canine shallow, ventral margin of premaxillary bone nearly horizontal; Lutreolina crassicaudata (FMNH 53944), premaxillary fossa for lower canine deep, ventral margin upturned, premaxillary bone slender, inclined. C, P. opossum (FMNH 114711), outer margins of nares steep, incisive distostyles obsolete or absent; Metachirus nudicaudatus (FMNH 20796), outer margins of nares gently sloping, nasal tips produced forward, distostyles present, the incisors trident.

Gryopus ovalis (São Paulo, Brasil; Hopkins, 1949)

Trimenopon hispidium (São Paulo, Brasil; Hopkins, 1949)

\section{Acarina (mites, ticks, chiggers)}

Archemyobia pectinata Méndez (Panamá; Méndez, 1972, p. 615)
Amblyomma auricularium Conil (Panamá; Fairchild et al., 1966, pp. 191, 209)

Amblyomma geayi Neumann (Panamá; Fairchild et al., 1966, pp. 195, 209)

Amblyomma sp. (Panamá; Fairchild et al., 1966, pp. 209; Venezuela; Jones et al. 1972)

Androlaelaps fahrenholzi Belese, 1911 (Venezuela; Furman, 1972) 


\section{The staggered marsupial incisor}

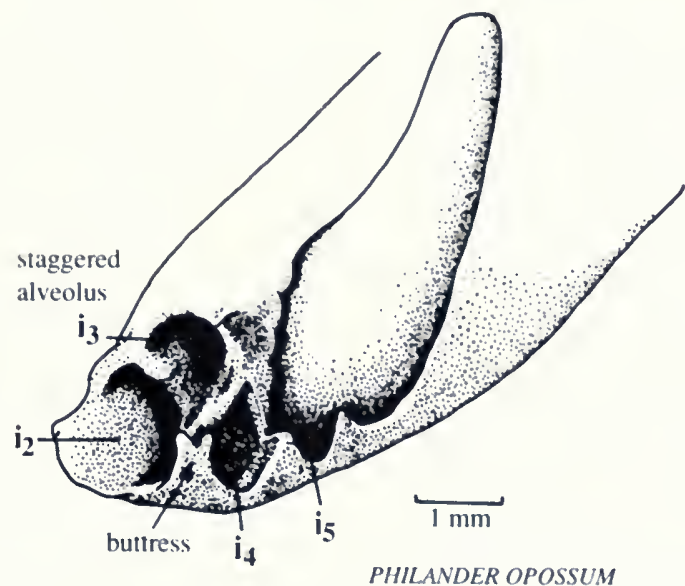

Fig. 16. Alveolus of staggered $i_{3}$, and buttress, in Philander opossum (from Hershkovitz, 1982).

Crotiscus disdentatus Boshell and Kerr (Panamá; Brennan \& Yunker, 1966, p. 260)

Pseudoschoengastia bulbifera Brennan (Panamá: Brennan \& Yunker, 1966, p. 260)

Trombicula dunni Ewing (Panamá; Brennan \& Yunker, 1966, p. 260)

Trombicula keenani Brennan and Yunker (Panamá: Brennan \& Yunker, 1966, p. 260)

Eutrombicula alfreddugesi Oudeman, 1910 (Venezuela; Brennan \& Reid, 1974; Panamá; Brennan \& Yunker, 1966, p. 260)

Eutrombicula goeldii Oudeman, 1910 (Venezuela; Brennan \& Reid, 1974; Panamá; Brennan \& Yunker, 1966, p. 260)

Eutrombicula tropita Ewing, 1925 (Venezuela; Brennan \& Reid, 1974)

Euschoengastia numezi Hoffmann (Panamá; Brennan \& Yunker, 1966, p. 260)

Ixodes lasallei Méndez and Ortíz, 1958 (Venezuela; Jones et al., 1972)

Ixodes luciae Senevet, 1940 (Venezuela; Jones et al., 1972)

Ixodes venezuelensis Kohls, 1953 (Venezuela; Jones et al., 1972)

Ixodes luciae Senevet (Panamá; Fairchild et al., 1966, p. 209)

Tur apicalis Furman and Tipton, 1961 (Venezuela; Furman, 1972)

Tur uniscutatus Turk (Panamá; Tipton et al., 1966, pp. 41, 42)

Ornithonyssus wernecki Fonseca (Panamá; Yunker \& Radovsky, 1966, p. 92)
Haemolaelaps glasgowi Ewing (Panamá; Tipton et al., 1966, p. 34)

Nematoda

Aspidodera sp. (Venezuela; Guerrero, 1985) Capillaria sp. (Venezuela; Guerrero, 1985)

Travassastrongylus sp. (Venezuela; Guerrero, 1985)

Moennigio sp. (Venezuela; Guerrero, 1985)

Strongyloides sp. (Venezuela; Guerrero, 1985)

Viannaia barusi Guerrero, 1983 (Venezuela: Guerrero, 1983)

Viannaia conspicua (Brasil; Imperial Bureau of Agricultural Parasitology, 1933, ex Potkay, 1977)

Rhopalia horridus (Angra dos Reis; Imperial Bureau of Agricultural Parasitology, 1933, ex Potkay, 1977)

Viannaia minispicula Guerrero, 1983 (Venezuela; Guerrero, 1983)

Viannaia skrjabini Lent and Freitas, 1937 (Venezuela: Guerrero, 1983)

Viannaia tenorai Guerrero, 1983 (Venezuela; Guerrero, 1983)

Viannaia viamniai Travassos, 1914 (Venezuela; Guerrero, 1983)

Cestoda

Linistowia iheringi (Brasil; Beveredge, 1982, p. 107)

Uniramia (Pterygota) 

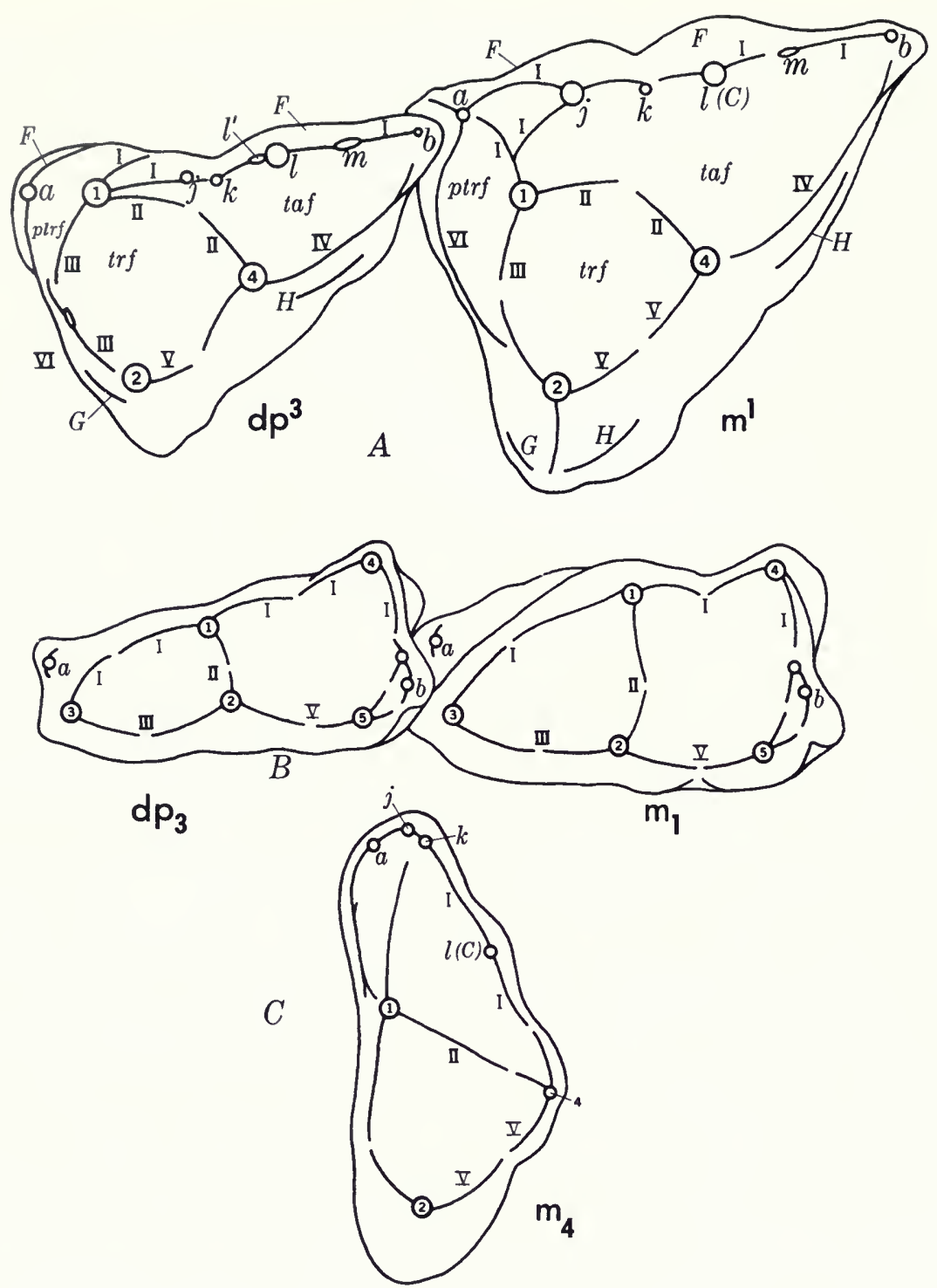

FIG. 17. Occlusal surface of deciduous premolar 3 and molars 1 and 4. A, Left $\mathrm{dp}^{3}$ and $\mathrm{m}^{1}$ of Philander opossum (FMnH 55411). B, Right $\mathrm{dp}_{3}$ and $\mathrm{m}_{1}$ of $P$. opossum (FMNH 66332). C, Left $\mathrm{m}^{4}$ (composite FMNH 90086, 14015).

\section{Coleoptera}

Amblyopinus henseli (Serra dos Órgãos, Angra dos Reis, Brasil; Tijuca; Seevers, 1955, p. 247)

\section{Siphonaptera (Fleas)}

Adoratopsylla intermedia intermedia Wagner, 1901 (Venezuela; Tipton \& Machado, 1972)
Adoratopsylla intermedia copha Jordan (Panamá; Tipton \& Méndez, 1966, p. 326; SW Colombia; Méndez, 1977, p. 166)

Adoratopsylla antiquorum cunhai Pinto (Brasil; Rio de Janeiro, Costa Lima \& Hathaway, 1946, p. 228)

Ctenocephalides felis Jordan and Rothschild (cosmopolitan; Costa Lima \& Hathaway, 1946, p. 213)

Xenopsylla cheopsis Rothschild (cosmopoli- 

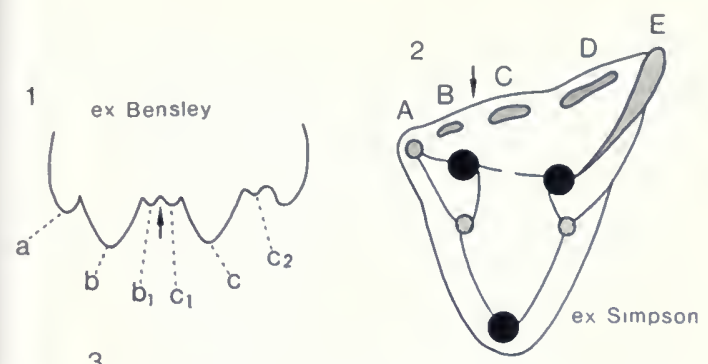

3

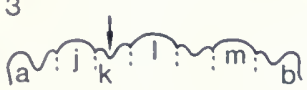

in text

FIG. 18. Conules (stylar cusps, ectostyles, terminal styles) of $\mathrm{m}^{3}$. 1, buccal view from Bensley (1906, p. 6); 2, occlusal view from Simpson (1929, p. 119); 3, buccal view from Hershkovitz (1977, p. 287); arrow points to ectoflexus. See Tables 6-7 for explanation of symbols.

tan; Costa Lima \& Hathaway, 1946, p. 228)

Polygenis roberti beebei I. Fox (Panamá; Tipton \& Méndez, 1966, p. 326)

Polygenis klagesi klagesi Rothschild (Brasil: Pará, Amazonas, Colombia; Panamá; Venezuela; Costa Lima \& Hathaway, 1946, p. 142)

Rhopalopsyllus australis tupinus Jordan and Rothschild (Panamá; Tipton \& Méndez, 1966, p. 326)

Rhopalopsyllus cacicus saevus Jordan and Rothschild (Panamá; Tipton \& Méndez, 1966, p. 326)

Rhopalopsyllus lutzi lutzi Baker (Argentina; Paraguay; Brasil: Goias, Minas Gerais; Costa Lima \& Hathaway, 1946, p. 139)

Neotyplocercus rosenbergi Rothschild (Ecuador: Perú; Costa Lima \& Hathaway, 1946, p. 229)

Tritopsylla intermedia intermedia Wagner (Brasil; Colombia; Ecuador; Paraguay; Central America; Costa Lima \& Hathaway, 1946, p. 228)

\section{Trematoda}

Duboisiella proloba Baer (Perú; Tantaleán et al., 1992) [Bursotrema tetracotyloides Szidat (Perú; Tantaleán et al., 1992)]

Zonorchis allentoshi Foster (Perú; Tantaleán et al., 1992)

Plagiorchis didelphidis Foster (Perú; Tantaleán et al., 1992)
Rhopalias caballeroi Kifune and Uyema (Perú; Tantaleán et al., 1992)

Rhopalias baculifer Braun (Perú; Tantaleán et al., 1992)

Paragonemus amazonicus Miyazaki, Grados, and Uyema (Perú; Tantaleán et al., 1992)

Rhopalias coronatus Rudolphi (Perú; Tantaleán et al., 1992)

Amphimeruse ruparupu Kifune and Uyema (Perú; Tantaleán et al., 1992)

Collins (1973, pp. 4, 74) listed the following genera of parasites of Philander without more information or regard for phylogenetic affinities. The list was culled from the "host catalog index card file of the Parasite Classification Index Catalogue of the United States Department of Agriculture's Parasite Control Center, Beltsville, Maryland."

Arthropoda

Adoratopsylla, flea

Amblyomma, tick

Ctenocephalides, flea

Crotiscus, chigger

Euschoengastia, chigger

Eutrombicula, chigger

Gigantolaelaps, louse

Gliricola, ${ }^{1}$ louse

Gyropus, ${ }^{1}$ louse

Haemolaelaps, mite

Heterothrombidium

Intercutestrix

Ixodes, tick

Leeuwenhoekia

Microthrombidium

Neothyphloceras,

flea

Ornithonyssus, mite

Pentastoma (see

Porocephalus)

Polygenis, flea

Porocephalus,

Pentastoma

Pseudoschongastia, chigger

Pulix, flea

Rhopalias

Rhopalopsyllus, flea

Schongastia

Trimenopon, ${ }^{1}$ louse

Tritopsylla, flea

Trombicula, chigger

Tur, Acarina

Xenopsylla, flea

Acanthocephala

Echinorhynchus

Hamanniella

Cestoda

Oorchoristica

Sparganum

Nematoda

Aspidodera

Capillaria

Cortiamosoides

Cruzia

Globocephalus

Gnathostoma

Gongylonemoides

1"All refer to one captive individual and are certainly due to contamination," Werneck, in Hopkins, 1949, p. 439 . 


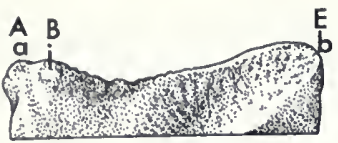

12010

P. opossum quica

Balta, Peru ơ (old)

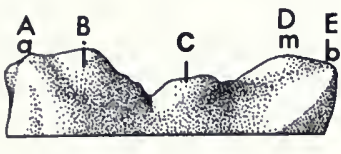

14012

$P$. opossum quica

Balta, Peru @ (yg)

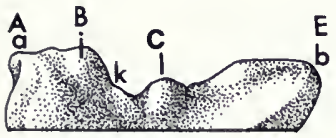

95313

P. opossum opossum Makerie, Suriname

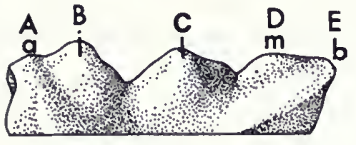

13799

P. opossum fuscogriseus Achotal, Mexico? (subad)

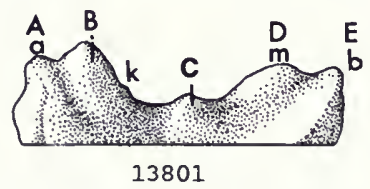

$P$. opossum fuscogriseus Achotal, Mexico ? (subad)

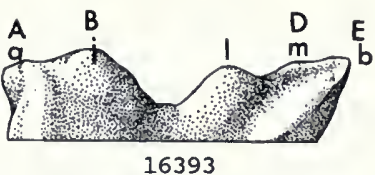

16393

$P$. andersoni andersoni Balta, Peru \& (adult)

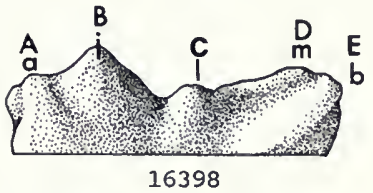

$P$. andersoni andersoni Huanhuachays, Peru o (adult)

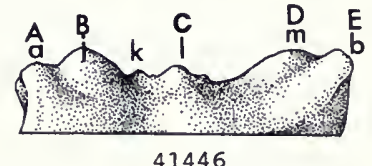

P. andersoni andersoni Montalvo, Ecuador of (adult)

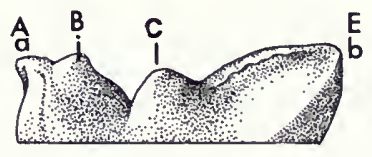

87123

P. andersoni andersoni Santa Luisa, Peru

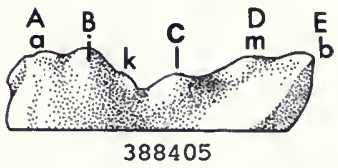

P. andersoni andersoni Rio Cunucunumá, Venezuela १ (subad)

FIG. 19. (FNNH) Conules of third upper molar labial shelf of Philander species from various geographic localities. See Table 6 for explanation of symbols.

$\begin{array}{lc}\text { Macielia } & \text { Sarcocystis } \\ \text { Oxysoma } & \text { Trypanosoma } \\ \text { Philostrongylus } & \text { Trematoda } \\ \text { Physaloptera } & \text { Brachylaemus } \\ \text { Skrjabinofilaria } & \text { Maritrema } \\ \text { Subulura } & \text { Opisthorchis } \\ \text { Travassostrongylus } & \text { Paragonimus } \\ \text { Trichuris } & \text { Phaneropsolus } \\ \text { Viannaia } & \text { Plagiorchis } \\ \text { Protozoa } & \text { Platynosomum } \\ \text { Besnoitia } & \text { Podospathalium } \\ \text { Haemogregaraina } & \text { Zonorchis }\end{array}$

\section{Species and Named and Unnamed Subspecies of Philander opossum}

The taxonomic accounts are arranged in the following order.

\section{Philander opposum Linnaeus}

Philander opossum opossum Linnaeus Philander opossum quica Temminck Philander opossum frenatus Olfers Philander opossum melanurus Thomas Philander opossum subspecies nov. 1 Philander opossum subspecies nov. ? 

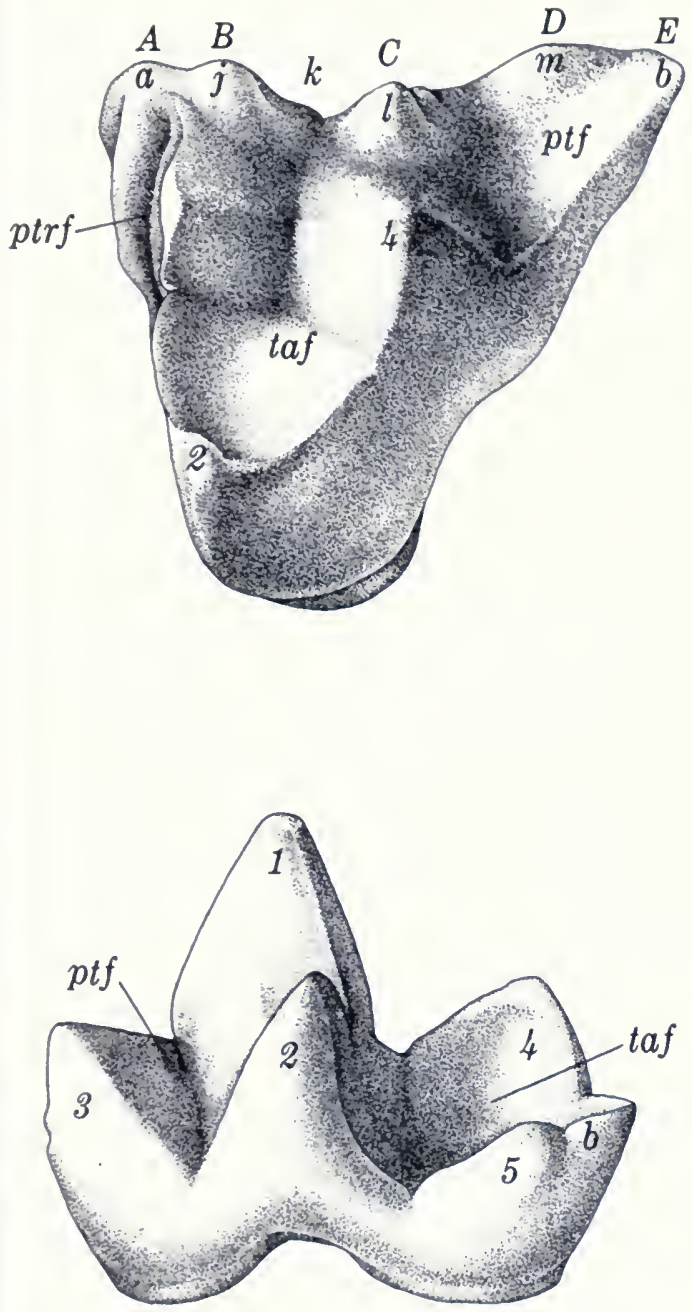

FIG. 20. Left upper third molar and right lower third molar of Philander opossum. See Table 6 for names of features.

\section{Philander opossum fuscogriseus}

J. A. Allen

2. Philander andersoni Osgood

Philander andersoni andersoni Osgood

Philander andersoni mcilhennyi

Gardner and Patton

\section{Philander opossum Linnaeus}

(Synonyms under subspecies heading)

Distribution (Fig. 5)-Distribution as for the genus, except replaced by $P$. andersoni in Amazonian Colombia and Ecuador and contiguous parts of Amazonian Perú, and in central Perú in the departments of Pasco, Junín, and Ayacucho. Philander opossum and $P$. andersoni are sympatric in the upper Rio Purús and mid and lower Río Ucayali basins.

HISTORIC VARIATION-The nearest putative ancestor of $P$. opossum was perhaps slightly smaller than its living descendants and possibly grayish agouti, likely marked by pale brown superciliary spots. The warm, humid Late Pleistocene climate of the ancestral habitat east of the Andes supported a dense tropical forest that permitted spread of the four-eyed opossum around the northernmost projections of the Andes to the Pacific coast and Middle America. With the onset of the cooler, drier climate of Late Pleistocene or Early Recent, shrinkage and fragmentation of the forest resulted in isolated refuges surrounded by superceding or invading savannas (Prance, 1982). The isolated populations of Philander differentiated into the racial forms recognized here. The most widely distributed or largest portion of the erstwhile continuously distributed ancestral population is $P$. opossum quica.

An undescribed population ( $P$. opossum subsp. nov. 1) of northeastern Venezuela (Fig. 5, f) is said to be very dark. The isolated $P$. opossum melanurus of coastal Ecuador is also dark. It remains to be seen if the ranges of $P$. opossum melanurus and $P$. opossum fuscogriseus meet on the west and between $P$. opossum quica and the undescribed $P$. opossum on the northeast.

The geographic range of the southeastwardspreading $P$. andersoni overlaps that of the westward-advancing $P$. opossum quica.

Cranial and Dental Characters-Cranial and dental characters as for the genus, comparisons included.

COloration (Fig. 4)-Color pattern of head as for the genus; cheeks, chin, throat, neck, chest, belly, inner sides of limbs ochraceous to buffy or whitish; marsupial region brown, scrotum unpigmented or partially to completely pigmented; lateral line, if present, usually confined to pelvic region as a hip patch, less frequently as a shoulder stripe or patch, color buffy to ochraceous orange, often appearing olivaceous when pale hair bases show through; crown, midline of nape, and dorsum to basal hairy portion of tail brownish; middorsal band, if present, poorly to strongly defined, individual cover hairs agouti (banded), tip brown (eumelanin), buffy (pheomelanin) subterminally, followed proximally by a brown band merging into curly and distinctly thinner grayish 
TABLE 6. Explanation of symbols used for marsupial dentition (Figs. 17-20).

\begin{tabular}{|c|c|}
\hline \multicolumn{2}{|c|}{ Upper teeth } \\
\hline \multicolumn{2}{|l|}{ Cones $^{1}$} \\
\hline 1 & Eocone (paracone) \\
\hline 2 & Protocone \\
\hline 3 & - \\
\hline 4 & Metacone \\
\hline 5 & - \\
\hline \multicolumn{2}{|c|}{ Conules and styles ${ }^{2}$} \\
\hline$a(A)$ & Mesiostyle- $a$ (parasty \\
\hline$b(E)$ & $\begin{array}{l}\text { Distostyle- } b \text { (metastyle; } \\
\text { hypoconule) }\end{array}$ \\
\hline$j(B)$ & Ectostyle $-j$ \\
\hline & Ectostyle- $k$ \\
\hline$l(C)$ & Ectostyle-l (mesostyle) \\
\hline$m(D)$ & Ectostyle- $m$ \\
\hline
\end{tabular}

Cristae $^{3}$

Cristae extend from-to or between cusps; reference cusps in parentheses are not elements of the indicated crista.

$I$
$I I$
$I I I$
$I V$
$V$
$V I$

$$
\begin{aligned}
& \text { Eocrista }[l-a-j-k-l-m-b] \\
& \text { Centrocrista }[1-4] \\
& \text { Epicrista [1-2] } \\
& \text { Postmetacrista [4-b] } \\
& \text { Plagiocrista (metaloph) [2-4] } \\
& \text { Protoloph (protocrista; crested } \\
& \text { portion of cingulum }(G)
\end{aligned}
$$

Basins or fossae

$\begin{array}{ll}\text { ptrf } & \text { Pretrigon basin or fossa } \\ \text { trf } & \text { Trigon basin or fossa } \\ \text { taf } & \text { Talon basin or fossa }\end{array}$

Cingula and cingulids Primary

$\begin{array}{cc}F & \begin{array}{c}\text { Buccal or external (buccal } \\ \text { shelf) } \\ \text { Anterolingual or anterior } \\ \text { (primary lingual shelf) } \\ \text { Posterolingual cingulum }\end{array} \\ & \text { H }\end{array}$

Upper and lower teeth

\section{Cristids $^{3}$}

Cristids extend from-to or between cuspids; reference cuspids in parentheses are not elements of the indicated cristid.

$\begin{array}{ll}I & \text { Eocristid [l-a-j-k-l-m-b]} \\ I I & \text { Centrocristid [l-4] } \\ I I I & \text { Epicristid [I-2] } \\ I V & \text { Postmetacristid [4-b] } \\ V & \text { Plagiocristid [2-4] } \\ V I & \text { Protocristid (crested portion } \\ & \text { of cingulum } B \text { ) }\end{array}$

Basins or fossids

$\begin{array}{ll}\text { ptf } & \text { "Trigonid" basin or fossid } \\ \text { taf } & -\end{array}$

Main enamel folds ex ectoflexus (between $1-4$ )

'Most cones and conids are numbered in the order of their origin and development.

${ }^{2}$ Most conules, conulids, styles, and stylids are listed in the order of their position from buccal to lingual and anterior to posterior. Rare or infrequent elements of the tritubercular trigon are listed opportunistically toward the end. Supernumeraries or gemini of established cusps and cuspids are not identified individually.

${ }^{3}$ Most cristae (-ids) are numbered in the order of their appearance or development in phylogeny, others are numbered opportunistically; all cristae (-ids) except $I-I$ inclusive are modified parts of cingula (ids); homologies of the talonid cristids are not certain in every case.

bases; guard hairs uniformly brownish; shoulders, sides of body, inner sides of limbs contrastingly paler than dorsum, the subterminal bands of cover hairs paler than those of dorsum, the dark guard hairs fewer and shorter; metapodials brown above, digits usually contrastingly paler or unpigmented, plantar surface usually well pigmented and darker than upper surface; tail naked in appearance except for hairy base, color entirely brown or mottled to particolored from tip to as much as distal three-fifths of tail more or less unpigmented; ears naked and wholly brown to nearly entirely unpigmented except for brownish borders.

Dichromatism - Gray and brown color phases described under the generic heading (p. 11) are present in $P$. opossum. In brown phase, the pheomelanic subterminal band of the cover hairs of dorsum and sides is saturate ochraceous or orange. The same band in the gray phase is bleached 
TABle 7. Molarization of conule $C$ in $\mathrm{m}^{3}$. a $=$ barely rudimentary; $b=$ rudimentary conule not always well defined; $\mathrm{c}=$ low distinct conule; $\mathrm{d}=$ small cusp; $\mathrm{e}=$ cusp comparable to conule $B$ in bulk. The tabulation is based on about 95 specimens in the Field Museum, plus about an equal number of borrowed specimens.

\begin{tabular}{|c|c|c|c|c|}
\hline Locality & $a-b$ & $b-c$ & $c-d$ & $d-e$ \\
\hline & \multicolumn{3}{|c|}{$\begin{array}{c}\text { Philander andersoni } \\
\text { andersoni }\end{array}$} & \\
\hline Venezuela & 8 & 8 & 4 & - \\
\hline Colombia & 1 & 1 & 2 & - \\
\hline Ecuador & 3 & 2 & 2 & - \\
\hline Perú (Andean) & 2 & - & - & - \\
\hline \multirow[t]{2}{*}{ Perú (Ucayali) } & - & - & 6 & 2 \\
\hline & \multicolumn{3}{|c|}{$\begin{array}{c}\text { Philander andersoni } \\
\text { mcillennyi }\end{array}$} & \\
\hline \multirow[t]{2}{*}{ Perú (Ucayali) } & - & - & 8 & 2 \\
\hline & \multicolumn{3}{|c|}{$\begin{array}{c}\text { Philander opossum } \\
\text { fuscogriseus }\end{array}$} & \\
\hline México & - & - & 3 & 6 \\
\hline Guatemala & - & - & 1 & 3 \\
\hline Honduras & - & - & - & 1 \\
\hline Costa Rica & - & - & 3 & - \\
\hline Panamá & - & - & 1 & 1 \\
\hline Colombia & 2 & 1 & 5 & 2 \\
\hline \multirow[t]{2}{*}{ Ecuador } & 1 & - & 1 & - \\
\hline & \multicolumn{3}{|c|}{$\begin{array}{c}\text { Philander opossum } \\
\text { melamurus }\end{array}$} & \\
\hline \multirow[t]{2}{*}{ Ecuador } & 2 & 2 & - & - \\
\hline & \multicolumn{3}{|c|}{$\begin{array}{c}\text { Philander opossum } \\
\text { opossum }\end{array}$} & \\
\hline Guianas & 4 & 2 & 1 & - \\
\hline \multirow[t]{2}{*}{ Brasil (Amazonian) } & 8 & 9 & 3 & - \\
\hline & \multicolumn{4}{|c|}{ Philander opossum quica } \\
\hline Eastern Brasil & 4 & 2 & 1 & - \\
\hline Bolivia & 12 & 7 & 3 & - \\
\hline Perú & 7 & 10 & 2 & - \\
\hline Venezuela & 2 & 5 & 8 & - \\
\hline
\end{tabular}

to pale buff or nearly white. Old pelage with tips and subterminal bands of hairs eroded, appears brown in either color phase because of greater exposure of the dark bases. Hairs of underparts in gray phase are dominantly bicolor with terminal portions pheomelanic, basal portions eumelanic. In brown phase, the hairs are dominantly monocolor pheomelanic.

Sexual dichromatism, as seen in $P$. andersoni, is not evident in P. opossum.

\section{Philander opossum opossum Linnaeus}

Philander, Opassum [sic], sive Carigueja, Brasiliensis: mas., Seba, 1734:56, Pl. 36, Fig. 1 (male [Fig. 21])—description.

Philander, Americanus, sive Carigueja, cum caulus, saccum ventris intrantibus foemina, Seba, 1734:57, Pl. 36, Fig. 2 (female with pouch young [Fig. 21])-description.

Carigueja, junior, sive Opassum [sic], cum sacco aperto, Seba, 1734:57, P1. 36, Fig. 3 (young female with open pouch)-description.

Sarigue, Buffon [and Daubenton], 1763:279, Pls. 4550 (animals, anatomy) - part, anatomical description of male (Pl. 45) and female (Pl. 46) only.

Molucca opossum, Shaw, 1800:476, Pl. 108.

[Didelphis] opossum, Linnaeus, 1758:55.

Didelphis opossum, Cuvier, 1798:114-le sarigue. É. Geoffroy St. Hilaire 1803:141—characters; habits. Latreille, 1803:304-sarigue ou opossum. Desmarest, 1820:256-characters. Temminck, 1825:41characters. Gray, 1843:101. Jentink, 1887:301SURINAME; GUYANE FRANÇAISE: (Cayenne). Jentink, 1888:220-SURINAME. J. A. Allen, 1900:195-SURINAME: (type locality).

Didelphis oposum [sic], Brongniart, 1792:115GUYANE FRANCAISE: (Cayenne).

Didelphys opossum, Goldfuss, 1809:215. Waterhouse, 1841:90-part, GUYANA; SURINAME. Waterhouse, 1846:485-GUYANA. Thomas, 1888: 329-part, GUYANA: Demerara (Better Hope); SURINAME. Lydekker, 1896:200-part, distribution; characters. Goeldi and Hagmann, 1904:100BRASIL: Pará; local name, mucurá chichica.

TABLE 8. Karyotypes of largest American marsupials.

\begin{tabular}{lcccccccc}
\hline \multicolumn{1}{c}{ Taxon } & $\mathbf{2 n}$ & SM & A & X & Y & FN & N $\$, 8$ & \multicolumn{1}{c}{ Source } \\
\hline Philander opossum & 22 & - & 10 & A & A & 20 & 7,9 & Reig et al. (1977) \\
Philander andersoni & 22 & - & 10 & A & A & 20 & 4,3 & Reig et al. (1977) \\
Didelphis marsupialis & 22 & - & 10 & A & A & 20 & 19,11 & Reig et al. (1977) \\
Didelphis albiventris & 22 & - & 10 & A & A & 20 & 3,7 & Reig et al. (1977) \\
Didelphis virginiana & 22 & 6 & 4 & M & A & 32 & 10,9 & Reig et al. (1977) \\
Chironectes minimus & 22 & - & 10 & A & A & 20 &,- 1 & Reig et al. (1977) \\
Lutreolina crassicaudata & 22 & - & 10 & M & A & 20 & 1,2 & Reig et al. (1977, p. 199) \\
\hline
\end{tabular}

$\mathrm{A}=$ acrocentric; $\mathrm{FN}=$ fundamental number of autosomal arms; $\mathrm{M}=$ metacentric: $\mathrm{SM}=$ submetacentric; $\mathrm{ST}=$ subtelocentric; $\mathrm{X}=q$ sex chromosome; $\mathrm{Y}=\delta$ sex chromosome; $2 n=$ diploid number of chromosomes; $N q, \delta$ $=$ number of $q$ and $\delta$ samples. 
TABle 9. Measurements of Philander opossum opossum Linnaeus.

\section{Locality}

Head and Body

Tail

Hind Foot

Ear

Philander opossum opossum

Guyana $\delta \delta^{*}$

All localities

Guyana $q$ ?

All localities

Suriname $\delta$ o

All localities

Suriname $q$ ?

Paramaribo

Guyane Française $\delta \delta$

Cayenne

Guyane Française $?$

Cayenne

Brasil oै के

Serra do Navio

Rio Amapari

Belém, Utinga

Capim

Baião

Guamá

Tapareba

Cametá

Taiuna

Santarém Highway

Altamira

Rosarinho

Imperatriz

Baptista

Brasil $q$ 우

Serra do Navio

Rio Amapari

Murutucu

Belém, Utinga

Mocajuba

Baião

Tapareba

Cametá

Taiuna

Santarém Highway
$-, 280,-, 312$
$254,287,-, 270$
$275(250-305) 8$

$245,277,260$

272,257

292

$302(280-320) 4$

260, 304

$280,270,280$

270,280

295,280

255

290

290 (260-314) 24

$270,270,280$

285

309

$-$

$-$

270

$-, 280$

-, 262, 290

260

268 (252-284) 6

273

290

275 (261-298) 11

255
$-, 298,-, 330$

$266,273,-, 264$

271 (254-310) 8

$262,264,269$

268,276

297

$315(310-320) 3$

280,300

$280,290,285$

290,320

295,305

280

$-$

300

289 (235-343) 24

$300,285,310$

- 85

321

$-$

275

$-, 258$

$-, 273,275$

265

279 (274-290) 5

270

350

298 (272-334) 11

290
$-, 42,-, 41$

$-, 38,-,-$

$41 \quad(40-42) 7$

$39,39,40$

$40,40,43$

42,41

39

44 (42-45) 4

42,50

$42,42,46$

42,42

41, 42

45

35

$45 \quad(41-50) 24$

$41,40,44$

35

50

$-$

40

$-, 35$

$-, 41,41$

35

$39.8(39-40) 5$

39

$-$

$42 \quad(41-43) 9$

40
$32,36,30$

37,34

41

35 (34-36) 4

34,42

$39,41,39$

36,36

$-$

40

$-$

-

$30,30,32$

$-$

$-$

-

35

$-, 20$

-, 38, 40

$-$

38

$-$

$-$
D[idelphys]. opossum, Olfers, 1818:204-part, synonymy. Wagner, 1855:226-listed.

Sarigua opossum, Muirhead, 1830(1819):429-classification.

Metachirus opossum, Burmeister, 1856:69, Pl. 11, Fig. 1 (skull)-characters. Thomas, 1901b:153GUYANA: Rupumuni (Rupununi River; Kanuku Mts.). Thomas, 1910:188-GUYANA: Demerara (Supinaam River). Thomas, 1911:143-SURINAME: (type locality); Seba sole reference for opossum Linnaeus. Sonntag, 1924:743, Figs. 39c, $40 i$ (tongue)-tongue anatomy. Boardman, 1952: 848 - hair tracts.

Metachirus opossum opossum, J. A. Allen, 1911: 246-VENEZUELA: Bolívar (Río Yuruán).

Didelphys (Metachirus) opossum, Ménégaux, 1902: 496-GUYANE FRANÇAISE: (Ouanary River).

Gamba opossum, Liais, 1872:329-classification.

[Metachirops] opossum, Matschie, 1916:268-SU-
RINAME: Paramaribo (type locality); classification.

Metachirops opossum opossum, Krumbiegel, 1941: 200_part, BRASIL: Pará (Ipitinga, Rio Acará; Peixe-Boi). Husson, 1978:24, Fig. 2c (front teeth), Pl. 1, Fig. 3 (hind foot), Pl. 5 (animal), Pl. 8 (skull)_SURINAME: Nickerie (Avanavero Falls, Kabalebo River; upper Nickerie River); Paramaribo (Paramaribo, various localities in and near town; Clevia; Lelydorp); Suriname (near Para River on road to Domburg, $10 \mathrm{~km} \mathrm{SE}$ of Paramaribo); Commewijne (near Commewijne River); Marowijne (Albina); characters; habits; vernacular names.

[Holothylax opossum] opossum, Cabrera, 1919:48classification.

Metacherius [sic] opossum, Sanderson, 1949:787, Pl. 7, Fig. I (hand), Fig. 2 (foot)-part, SURINAME; local names, fructu-awari, awari.

Philander opossum, Gilmore, 1941:309-classifica- 


\section{Condylobasal}

Length

\section{Zygomatic Breadth}

Preorbital Width

\begin{tabular}{|c|c|c|c|c|}
\hline $71.7(68.4-75.4) 4$ & $34.1,-, 34.6,38.9$ & $12.3,-, 12.9,-$ & $9.1,-, 8.4,8.5$ & $20.3,18.4,22.5$ \\
\hline $66.0,-69.7,70.3$ & $31.4,-, 36.5,36.0$ & $-,-, 13.4,12.5$ & $-,-, 8.1,8.5$ & $19.6,-, 19.2,19.6$ \\
\hline $70.6(67.3-73.3) 7$ & $36.9(33.8-39.1) 6$ & $12.8(11.5-14.6) 7$ & $8.4(7.5-9.2) 7$ & $20.7(19.4-21.3) 7$ \\
\hline $69.3,-,-$ & $35.7,-,-$ & $12.8,-,-$ & $8.0,-,-$ & $20.8,-,-$ \\
\hline $69.5,73.1$ & $38.3,38.5$ & $13.4,13.4$ & $9.4,8.8$ & $20.6,19.7$ \\
\hline 68.2 & 35.3 & 12.2 & 9.7 & 19.7 \\
\hline $\begin{array}{l}74.1(69.7-82.1) 20 \\
69.4,77.1 \\
68.2,70.0,74.6 \\
70.0,74.9 \\
73.2(69.4-75.5) 4 \\
75.1 \\
69.8,70.4 \\
72.1 \\
74.2(68.4-78.0) 20 \\
70.4,70.2,73.3 \\
72.5 \\
65.4 \\
79.0 \\
72.9\end{array}$ & $\begin{array}{l}38.7(35.3-43.4) 20 \\
34.5,39.8 \\
34.7,33.0,38.7 \\
33.0,39.1 \\
37.3(36.3-38.6) 4 \\
37.2 \\
38.7,37.1 \\
39.4 \\
39.5(35.9-42.3) 20 \\
35.3,36.6,39.3 \\
35.7 \\
35.0 \\
43.0 \\
34.5\end{array}$ & $\begin{array}{l}14.4(12.5-15.3) 20 \\
12.5,18.9 \\
12.4,-, 13.0 \\
-.13 .3 \\
- \\
- \\
16.1,16.2 \\
17.6 \\
14.2(12.3-15.7) 19 \\
12.9,-,- \\
- \\
- \\
- \\
-\end{array}$ & $\begin{array}{l}8.9(8.2-9.5) 20 \\
8.6,9.4 \\
8.8,8.5,8.8 \\
8.5,8.9 \\
8.7(8.5-9.1) 4 \\
9.0 \\
8.2,8.0 \\
9.2 \\
8.8(8.4-9.5) 20 \\
8.9,9.3,9.7 \\
9.0 \\
8.5 \\
8.9 \\
8.8\end{array}$ & $\begin{array}{l}21.8(20.5-22.9) 20 \\
19.3,21.7 \\
20.3,19.7,21.4 \\
19.7,22.1 \\
21.9(21.4-22.0) 4 \\
20.9 \\
- \\
21.7 \\
20.9(19.3-22.9) 20 \\
20.7,20.2,21.4 \\
21.5 \\
21.3 \\
24.0 \\
19.3\end{array}$ \\
\hline $\begin{array}{l}75.7(69.1-80.8) 4 \\
75.5 \\
69.7,72.1 \\
69.5,70.9,74.3 \\
64.8 \\
66.6(64.0-69.2) 7 \\
67.0 \\
76.4 \\
69.2(65.4-75.5) 13 \\
65.4\end{array}$ & $\begin{array}{l}- \\
39.0 \\
32.2,36.2 \\
34.6,35.1,36.5 \\
32.5 \\
32.6(31.3-34.0) 6 \\
31.1 \\
36.8 \\
35.0(32.1-40.7) 13 \\
32.8\end{array}$ & $\begin{array}{l}- \\
18.4 \\
-, 12.9 \\
13.0,12.4,13.7 \\
- \\
- \\
- \\
17.7 \\
12.5(11.4-13.7) 13 \\
-\end{array}$ & $\begin{array}{l}- \\
9.0 \\
8.7,9.0 \\
8.2,8.5,9.0 \\
8.1 \\
8.9(8.3-9.2) 7 \\
8.8 \\
9.0 \\
8.7(8.1-9.1) 13 \\
9.2\end{array}$ & $\begin{array}{l}- \\
20.4 \\
19.5,19.4 \\
20.0,20.0,20.8 \\
18.9 \\
20.0(19.3-20.8) 7 \\
19.3 \\
21.4 \\
19.9(19.2-21.4) 13 \\
20.2\end{array}$ \\
\hline
\end{tabular}

tion. Pine, 1973:51 (key), 56-BRASIL: Pará (Utinga, Belém). Miles et al., 1981a:331-BRASIL: Pará (Belém); tracking; habitat; comparison with Metachirus nudicaudatus. Miles et al., 1981 b: 272-BRASIL: Pará (Belém); habitat; host of triatomine bugs vectors of Trypanosoma cruzi. Charles-Dominique et al., 1981:342—GUYANE FRANCAISE: Cayenne; habitat; behavior, diet. Atramentowicz, 1986a:123 GUYANE FRANCAISE: Cayenne; habits; reproduction. CharlesDominique, 1983:395-422-GUYANE FRANCAISE: Cayenne; habits, habitat; reproduction. Guerrero, 1985:41-VENEZUELA: parasites. Atramentowicz, 1988:47-GUYANE FRANCAAISE: Cayenne (frugivory).

Philander opossıın opossum, Ávila-Pires, 1958:4 BRASIL: Pará (Utinga, Belém de Pará). Husson, 1978:5-SURINAME: local names, fo-ai awari, vieroog-opossum, kaalstaart. Carvalho, 1961:5-
BRASIL: Rio Branco (Poçâo, Caracarai; Poçâo, Bôa Vista); habits; disagrees with Sanderson's reports. Carvalho, 1962:285-BRASIL: Amapá (Rio Amapari, Macapá; Rio Maracá, Mazagão; Rio Branco, Rio Maracá, Mazagão); local name, mucura xixica. Pine, 1973:56-BRASIL: Pará (Belém; Utinga); characters. Pérez-Hernández, 1989: 373-VENEZUELA: Bolivar (El Palmar; San Martín de Turumbarin; Chalunani).

Philander o[possum]. opossum, Carvalho and Tocheton, 1969:217-BRASIL: Pará (Utinga, Belém; Sapucajuba, Belém; km 94 Belém-Brasília); local name, mucura xixica.

P[hilander]. virginianus Tiedemann, 1808:427-replacement name for $D$. opossum Linnaeus.

Didelphis austro-americana Thomas, 1923:604name ex Oken 1816:1148-a non-Linnaean work; synonym of $D$. opossum Linnaeus. 


\section{Locality}

Palatal Length

$\mathrm{i}-\mathbf{m}^{4}$

$m^{1-4}$

Philander opossum opossum

Guyana $\delta$ के

All localities

Guyana $q$ ?

All localities

Suriname $\delta \hat{\sigma}$

All localities

Suriname $q$ 우

Paramaribo

Guyane Française $\delta \delta$

Cayenne

Guyane Française $q$

Cayenne

Brasil oै कै

Serra do Navio

Rio Amapari

Belém, Utinga

Capim

Baião

Guamá

Tapareba

Cametá

Taiuna

Santarém Highway

Altamira

Rosarinho

Imperatriz

Baptista

Brasil $q$ 우

Serra do Navio

Rio Amapari

Murutucu

Belém, Utinga

Mocajuba

Baião

Tapareba

Cametá

Taiuna

Santarém Highway

$43,1(41.6-44.1) 4$
$40.7,-, 43.0,43.7$
$43.0(41.8-43.8) 7$
$43.1,-,-$
$41.8,45.8$
41.0
$45.1(41.7-49.6) 20$
$42.1,46.2$
$42.3,41.3,44.2$
$41.3,42.9$
$44.0(41.6-46.7) 4$
44.4
$40.8,40.3$
42.6
$44.3(42.0-46.4) 20$
$41.8,42.4,43.9$
44.0
40.3
47.8
44.9
-
45.7
$43.6,43.3$
$42.0,42.3,44.7$
38.4
$40.7(38.4-42.9) 7$
41.4
45.8
$41.7(39.2-44.8) 13$
40.3

43,1 (41.6-44.1) 4

$38.0(36.9-38.8) 4$

$36.5,-, 37.8,38.8$

$37.4(36.5-38.1) 7$

$37.9,-,-$

$36.9,39.2$

36. 1

38.9 (36.8-40.3) 20

$36.7,39.8$

$36.7,36.0,38.8$

$36.0,37.6$

$38.5(37.0-41.5) 4$

38.2

$35.8,37.7$

37.7

$38.2(37.2-40.5) 20$

$36.8,37.2,38.4$

37.7

34.9

40.9

37.7

- 38.6

$36.9,37.6$

$37.3,36.5,38.3$

34.8

$36.0(35.1-36.9) 7$

36.8

39.3

36.7 (35.0-38.1) 13

35.6
$14.1(13.6-14.5) 4$

$14.0,-, 14.0,14.7$

$13.8(13.4-14.4) 7$

$14.0,14.0,-$

$13.9,14.2$

14.0

$14.1(13.6-14.9) 20$

$14.0,14.0$

$13.4,13.6,13.7$

$13.6,13.3$

$13.9(13.0-14.1) 4$

14.0

$13.7,13.8$

14.5

$13.4(12.8-14.3) 20$

$13.6,13.4,14.3$

13.7

14.1

14.1

13.1

14.0

13.8

$12.9,13.7$

$14.0,13.5,13.6$

13.4

$13.5(13.0-14.3) 6$

13.4

14.0

$13.6(12.7-14.0) 13$

13.1
Didelphis Marsupialis, Shaw (not Linnaeus), 1800: 476, Pl. 108-Molucca opossum.

Didelphis quica, Jentink (not Temminck), 1888:220part, SURINAME. Schomburgk 1840:344-GUYANA; characters; habits.

Didelphys quica, Waterhouse, 1841:90-part, GUYANA; SURINAME. Waterhouse, 1846:480-part, GUYANA; SURINAME.

D[idelphys]. quica, Cabanis (not Temminck), 1848, 3: 777 -GUYANA.

Metachirops opossum quica, Carvalho (not Temminck), 1958:123-BRASIL: Pará (Gradáus, Rio Fresca); local name, mucura xixica.

Types (Fig. 21)-Name based on Seba's (1734) "Philander Opassum, sive Carigueja, Brasiliensis: mas." and "Philander, Americanus, sive Carigueja, cum catulus, sacrum ventris intranti- bus foemina"; the female, in alcohol, designated lectotype by Hershkovitz (1976), is preserved in the Rijksmuseum van Naturlijke Historie, Leiden.

TYPE LOCALITY-Suriname, determined by $\mathbf{J}$. A. Allen $(1900$, p. 195), further restricted to Paramaribo by Matschie (1916, p. 262).

Distribution (Fig. 5)-Guyana, Suriname, Guyane Française; in Brasil the states of Amapá, Roraima, and equatorial parts of Pará and Amazonas, in the Amazonian basin west of the Rios Tocantins-Araguaia. The hiatus between the west bank of the Rio Negro, north bank of the Rio Solimõés and eastern slope of the Andes cannot be attributed to lack of suitable habitat, negative collecting, or preemption of parts of the range by 


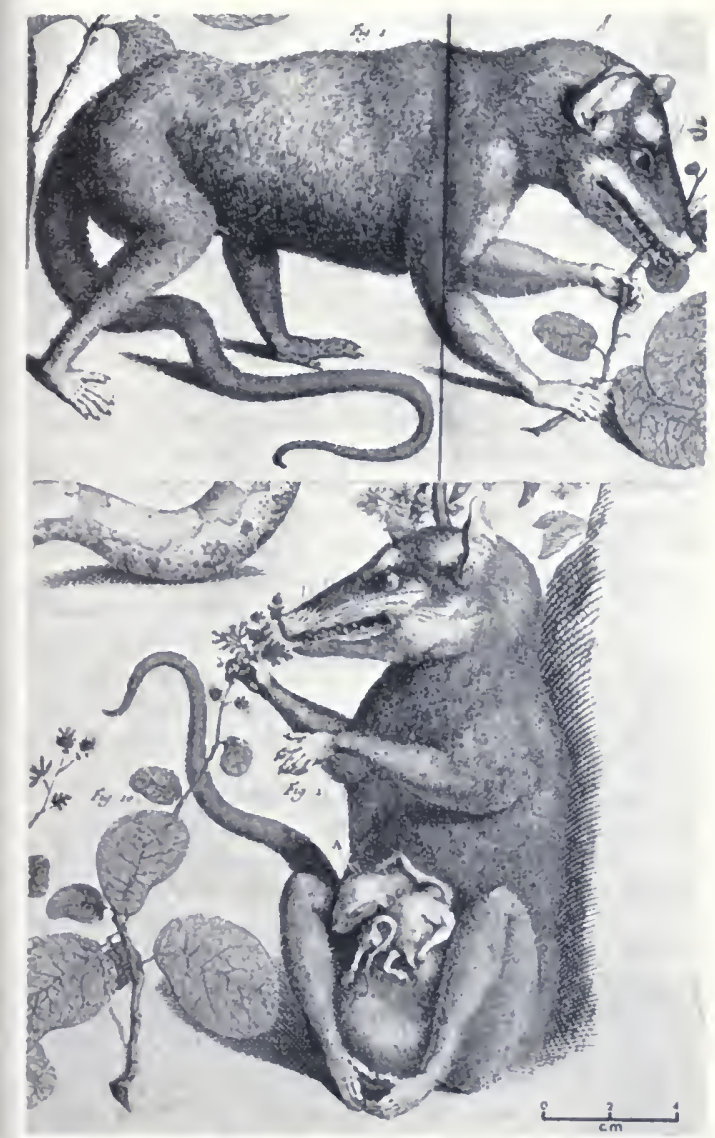

Fig. 21. Male and female lectotypes of Didelphis opossum Linnaeus (1758). Upper, "Philander opossum sive Carigueja Brasiliensis mas," the male opossum. Lower, "Philander americanus, sive Carigueja, cum catulus, Sacrum ventris intrantibus foemina." Figures and quotations from Seba (1734, p. 57, Pl. 36, Fig. 2).

P. andersoni. It may be that during a Pleistocene climatic change, the warm, humidity-loving opossum disappeared from even more extensive areas where savannas replaced forests.

Characters-General body size larger throughout than quica or melanurus; molars proportionately smaller; upper parts of trunk grayish to buffy brown, under parts buffy orange to ochraceous orange; tail usually parti-colored.

Comparisons-Few individuals of $P$. opossum opossum are as small as average-sized quica, and the underparts of none of the specimens at hand show the whitish, pale buff or grayish brown underparts present in many individuals of other races.

Measurements-Tables 1 and 9.
Taxonomy-Sole basis for the Linnaean $D i$ delphis opossum is the animals described by the Dutch pharmacist and naturalist collector Albert Seba (1734, in the work entitled Locupletissimi rerum naturalium thesauri . . , 1, p. 56, PI. 36, Fig. 1 [male], Fig. 2 [female]). Upper parts of the body of the male four-eyed opossum shown in the cited figure reproduced here (Fig. 21) are described as dark chestnut, and the tail base is said to be furred for the length of a human finger, or roughly between 7 and $9 \mathrm{~cm}$. Seba's female, Figure 2 (Fig. 21 here), is fully pouched, but no formal description of the animal appears in the text. Linnaeus (1758, p. 55), in citing Seba, evidently intended that the name $D$. opossum apply to the female. His diagnosis and description, " $D$ [idelphis]. cauda semipilosa, superciliorum regione pallidore, mammis binis" and "abdomen circa mammas contrahitur in marsupium; policis postici mutici," leaves no doubt. Color is not mentioned, but the "cauda semipilosa" can be variously translated as hairy for half its length or as partially furred. Whatever the wording, the interpretation must be derived from Seba's woodcut figure of the female (Fig. 21).

VARIATION-The types of $P$. opossum opossum Linnaeus, as described by Seba $(1734$, pp. 56,57$)$ are brown-phase individuals. With respect to other Suriname specimens, a male I collected in La Poule and another in Clevia, Paramaribo, are brown phase and resemble the lectotype preserved in alcohol in the Leiden Museum. A young female taken in Lelydorpplan is gray phase. One of two individuals collected by $\mathrm{H}$. A. Beatty in the Wilhelmina Mountains is gray phase, the other brown phase. Other Suriname skins examined are of juvenals.

Gray- and brown-phase individuals and intermediates occur throughout the range of the race. In all, however, underparts are more densely pigmented than in those of neighboring $P$. oposstum quica. The large series from the Rio Tocantins (Baiao; Ilha do Taiuna) exhibit the full range of color variation in $P$. opossum opossum.

REMARKS-Under the name Metacherius [sic] opossum, Sanderson (1949, p. 787) recorded 10 males and 12 females from Suriname. Of the total, 16 are said to have been taken in Paramaribo, and one, a large female, from Zanderij. Other specific localities are not mentioned. I found skins of six males and three females in the British Museum, all labelled Paramaribo, collected by I. T. Sanderson. There is also the female from Zanderij (BM 52.122), but it proves to be Metachirus nudicau- 
datus. Its mammae and a sketch of the mammary formula are by Sanderson (1949, Pl. VII, Figs. 3, 4). The remaining 12 of the 22 collected by Sanderson are not among the skins in the British Museum. Husson (1978, p. 28) mentions only the Zanderij Metachirus collected by Sanderson but no Philander.

Specimens Examined-370. BRASIL. Amapá: Ferrovia Amapá, km 192, 1 (LSUMZ); Macapá, Rio Amapari, 3 (MZUSP); Mazagão, Rio Maracá, 1 (MZUSP); Serra do Navio, 59 (USNM, 57; MPEG, 2); Terezinha, Rio Amapari, 1 (LSUMz); Amazonas: Auara Igarapé, 2 (AMNH); Ipixuna, Rio Purús, 1 (USNM); Lago do Baptista, I (AMNH); Lago Sampaio, Rosarhino, 2 (AMNH); Rio Madeira, 1 (AMNH); "River Negro," 5 (BMNH); Santo Antônio de Uayara, 1 (AMNH); Santa Clara, Villa Bella Imperatriz, 3 (AMNH); Tefé, 1 ( AMNH); Pará: Altamira, $54 \mathrm{~km} \mathrm{S,} 150 \mathrm{~km} \mathrm{W,} 3$ (USNM); Altamira, Rio Xingú, 3 (USNM); Ananindeua, 2 (MPEG); Arumatheua, 1 (MPEG); Baiao, Rio Tocantins, 14 (AMNH); Belém 2 (AMNH, 1; USNM, 1); Cametá, 17 (MZUSP); Canudos, 1 (FMnH); Capim, 6 (AMNH, 5; MPEG, 1); Cuatipuru, Flor do Prado, 2 (MPEG); Fordlandia, 1 (AMNH); Gradaus, Rio Fresco, 2 (MZUSP); Igarapé Tapereba, 4 (MPEG, 2; MZUSP, 2); Ilha do Taiuna, 85 (AMNH); Ipeau-Apez, Belém, 2 (USNM); Iriteria, 1 (MPEG); Lazaropolis, 4 (MPEG); Macajuba, Rio Tocantins, 3 (AMNH); Marabá, Rio Tocantins, 2 (USNM); Marcos, 1 (MPEG); Mazagão, Rio Tocantins, 1 (MPEG); Murutucú, 5 (BM, 2; FMnH, 3); Peixe-Boi, 1 (MPEG); Rodovia BelémBrasília, 3 (MPEG); Santa María, Bragança, 1 (USNM); Santarém, 10 (MZUSP, 2; USNM, 8); San Miguel do Guamá, 5 (MPEG, 2; MZUSP, 3); Utinga, 22 (MPEG, 6; USNM, 13; AMNH, 1; MZUSP, 2); Roraima: Caracaraí, 2 (MZUSP). GUYANA. Essequibo Islands-West Demerara: Buckhall, 2 (BM[NH]); Upper Takutu-Upper Essequibo: Kanuku Mts., 5 (BM[NH]); Rupununi River, 1 (BM[NH]); Demerara-Mahaica: Better Hope, 1 ( $\mathrm{BM}[\mathrm{NH}])$; Georgetown, 2 (FMNH); Hyde Park, 4 (BM[NH], 2; FMNH, 2); Pomeroon-Supenaam: Supenaam River, 1 (BM[NH]). GUYANE FRANÇAISE. Guyana: Cayenne, 5 (BM[NH], 1; FMNH, 4). SURINAME. Suriname: Paramaribo, Clevia, 2 (FMNH); Lelydorpplan, 1 (FMNH); Paramaribo, 10 (BM[NH], 9; lectotype, RMNH); no precise locality, 3 (BM[NH]); Nickerie: Kaiserberg Airstrip, 1 (FMNH); Makerie, West River, 2 (FMNH); Saramacca: La Poule, 1 (FMNH); Loksie Hattie, 1 (FMNH). VENEZUELA. Bolivar: Ciudad Bolívar, $146 \mathrm{~km} \mathrm{S,} 7 \mathrm{~km} \mathrm{NE}, 2$ (USNM); Maripa, 1 (AMNH); Río Yuruán, 10 (AmNH); Trujillo: Agua Viva, $18 \mathrm{~km} \mathrm{~N}$ Valera, 1
(USNM); El Dividiri, 30 km NW Valera, 1 (USNM); Motatin, 1 km NNE, 3 (UKMNH); Motatá, 5 km NNE, 1 (UKMNH); Río Motatin, 5 (UKMNH).

\section{Philander opossum quica Temminck}

Didelphis quica Temminck, 1824:36. Desmarest, 1827:387-characters. Jentink, 1887:301-BRASIL: Temminck collection. Jentink, 1888:220part, BRASIL; cotype; Didelphis larvata ms. name in synonymy. Hochstetter, 1946:11, Pl. 1, Fig. 2 (brain, sagittal section in skull)-brain, dura mater. Ávila-Pires and Gouvea, 1977:9-BRASIL: Rio de Janeiro (Monte Serrat; Benfica; Maceiras); local name, guaquica.

Didelphys quica, Waterhouse, 1841:90-part, BRASIL. Waterhouse, 1846:480-part, BRASIL. Burmeister, 1854:136-BRASIL: Rio de Janeiro (Novo Friburgo). Pelzeln, 1883:110-BRASIL: Rio de Janeiro (Sepetiba); São Paulo (Ypanema); Mato Grosso (Mato Grosso). Oudemans, 1892:14, Fig. 25 (Cowper's gland)-male accessory reproductive organs.

$D$ [idelphys]. quica, Wagner, 1855:225, Pl. 18 (female)-description, figure, and notes from Natterer manuscript.

Metachirus quica, Burmeister, 1856:70, Pl. 7 (male), p. 11, Fig. 2 (skull)—part, BRASIL: Rio de Janeiro (Novo Friburgo); characters. Hensel, 1872:120BRASIL: Rio Grande do Sul; characters. Goeldi, 1894:460-BRASIL: Rio de Janeiro (Serra dos Órgãos). J. A. Allen, 1900:195-BRASIL: (type locality, "coast region of Brasil, just south of Rio de Janeiro"').

Metachirus opossum quica, J. A. Allen, 1916c:562BRASIL: Mato Grosso (Urucum); Rio de Janeiro (Sapitiba [ = Sepetiba], restricted type locality). Miller, 1916:589-BRASIL; habits. Carvalho, 1957:3-BRASIL: Acre (Seringal Oriente, Rio Juruá).

[Metachirops] quica, Matschie, 1916:268-BRASIL: Rio de Janeiro (type locality, "Sapitibi").

Metachirops quica, Pohle, 1927:243-BRASIL: Rio de Janeiro (Barreira; Teresópolis). Schirch, 1932: 85-BRASIL: Rio de Janeiro (Teresópolis, 960 m); parasite (Amblyopinus).

Metachirops opossum quica, Miranda Ribeiro, 1936: 340-BRASIL: Rio Grande do Sul (Porto Feliz, Rio Urugaí); Rio de Janeiro (Teresópolis); Santa Catarina. Vieira, 1945:421-BRASIL: Mato Grosso (Palmeiras). Vieira, 1949:345-BRASIL: São Paulo; Minas Gerais; Espírito Santo; Mato Grosso; local names, quica, quaiquica. Ruschi, 1965:2BRASIL: Espírito Santo (coast); local names, cuica, chupata.

Philander opossuin quica, Perondini and Perondini, 1966:28-BRASIL: São Paulo (Cantarera Forestal Preserve); sex chromatin in somatic cells. Carvalho, 1965:251-BRASIL: São Paulo (Boraçeia).

[Holothylax] quica, Cabrera, 1919:48-classification.

D[idelphys]. quica [sic], Giebel, 1859:227—“der Guica.'

Didelphis myosuros Temminck, 1824:38-BRASIL: (type locality); cotypes in the Leiden, Vienna, 
Frankfort, and Prince Maximilian zu Wied-Neuwied Museums. Hershkovitz, 1959:343-in synonymy of Philander opossum frenata [sic] Olfers; original description based on four-eyed pouched opossum.

Metachirus nudicaudatus myosurus [sic], Miranda Ribeiro, 1936:345-BRASIL: Bahia; characters; Portuguese translation of original description: "... as femeas tem um sacco completo...".

Didelphis lanata Jentink, 1888:220-Natterer ms. name on label of a syntype of quica Temminck.

Metachirus canus Osgood, 1913:96-PERÚ: San Martin (Moyobamba, type locality); holotype, male, skin and skull, Field Museum of Natural History, no. 19347; collected 4 August 1912 by W. H. Osgood and M. P. Anderson. Osgood, 1914:148PERÚ: San Martín (Moyobamba). Sanborn, 1947: 215-type history.

[Metachirops] canus, Matschie, 1916:268-classification.

[Holothylax grisescens] canus, Cabrera, 1919:47classification.

Metachirus opossum camus, Thomas, 1927a:372PERÚ: San Martín (Rioja; Moyobamba; Yurac Yacu). Thomas, 1927b:606-PERÚ: Huánuco (Tingo María, $2000 \mathrm{ft}$ ). Thomas, 1928a:264PERÚ: Ucayali (Cumeria, $1000 \mathrm{ft}$; Chicosa, 1000 $\mathrm{ft}$; San Jerónimo, $500 \mathrm{ft}$ ).

M[elachirus]. o[possum]. canus, Thomas, 1928b: 294 -crucialis Thomas a synonym.

Philander opossum canus, Sanborn, 1949:277PERÚ: Ucayali (Yarinacocha). Sanborn, 1951:2PERÚ: Cuzco (Hacienda Cadena). Cabrera, 1958: 35-crucialis Thomas a synonym.

Metachirus opossum azaricus Thomas, 1923:604 PARAGUAY: Paraguari (Sapucay, type locality): holotype, female, skin and skull, British Museum (Natural History), no. 1903.2.3.36; collected $8 \mathrm{Au}-$ gust 1892 by W. Foster.

Met[achirops]. opossum azaricus, Krumbiegel, 1941: 203, 206-PARAGUAY: (Lapango); BRASIL: Rio Grande do Sul (Passo Fundo); BOLIVIA: Chiquitos (San Ramón); crucialis Thomas a synonym.

Philander opossum azaricus, Cabrera, 1958:34 classification. Kantis, I963:54-ARGENTINA: Chaco (Río de Oro, mouth); female with three young. Crespo, 1974:3-ARGENTINA: Chaco (Río de Oro, mouth); Misiones (Fracrán, San Pedro).

Metachirus opossum crucialis Thomas, 1923:604 BOLIVIA: Santa Cruz (type locality, Santa Cruz de la Sierra); holotype, female, skin (originally mounted) and skull, British Museum (Natural History), no. 1847.11.22.15; collected by Thomas Bridges.

Didelphys opossum, Waterhouse, 1841:90-part, BRASIL. Waterhouse, 1846:485-part, BRASIL. Thomas, 1888:329-ARGENTINA: Chaco; BRASIL: Rio Grande do Sul (Taquara); BOLIVIA: Santa Cruz (Santa Cruz de la Sierra). Winge, 1893: 7, 38, Pl. 1, Fig. 7 (ear, foot), PI. 3, Fig. I (skull), PI. 4, Fig. 7 (humerus)-BRASIL: Minas Gerais (Lagoa Santa, Recent and fossil); characters; comparisons.

D[idelphys]. opossum, Tschudi, 1844:14, 144, 151PERÜ.

Metachirus opossum, Ihering, 1892:99-BRASIL:
Rio Grande do Sul; local names, guaquica, guaqui. Ihering, 1894:10-BRASIL: São Paulo. Thomas, 1902a:64-BRASIL: Paraná (Roça Nova, Serra do Mar). J. A. Allen, 1916b:201-COLOMBIA: Meta (Villavicencio). Bertoni, 1923:5 I-PARAGUAY: Central (Bahía de Asunción); Guaraní name, guaki. M[etachirus]. opossum, Miranda Ribeiro, 1905:189BRASIL: Rio de Janeiro (Monte Serrat, Itatiaya).

Metachirops opossum, Bertoni, 1939:6-PARAGUAY. Crespo, 1950:6, Fig. I (animal)-ARGEN. TINA: Misiones (Río Urugua-í, $39 \mathrm{~km}$ from Puerto Bemberg); habits; locomotion; local name guaitica.

Philander opossum, Davis, 1945a: 122-BRASIL: Rio de Janeiro (Fazenda Boã Fe, Teresópolis). Davis, 1947:1 - BRASIL: Rio de Janeiro (Fazenda Boã Fe, Teresópolis); life history. Moojen and ÁvilaPires, 1966:397-BRASIL: cerrado formation (absent; Didelphis and Monodelphis only). Gardner and Patton, 1972:5-PERÚ: Ucayali (Balta, Yarinacocha); comparisons with mcilhennyi. Reig et al., 1977:197, 212, Pl. I (karyotype)-part, PERÚ: Ucayali (Yarinacocha; Balta). Mello and Moojen, 1979:289-BRASIL: Distrito Federal (Brasília gallery forest; Fazenda Agua Limpa, gallery; Guara, gallery); Mato Grosso (Baliza, gallery; Barra do Garças gallery; Poconé gallery; Salobra gallery); Goiás (Aragarças gallery; Formosa gallery). Correa Gomes, 1984:369-BRASIL: Mato Grosso (Salobra); helminth infection. Da Fonseca and Redford, 1984:517-BRASIL: Distrito Federal (Parque Nacional, Brasília; humid gallery forest and valley side wet campo; cerrado [uncommon]). Mares et al., 1989:19-BRASIL: Mato Grosso (Poconé).

Philander opossum opossum, da Fonseca and Kierulff (not Linnaeus), 1989: 118, 143-BRASIL: Minas Gerais (Fazenda Esmeralda; Fazenda Montes Clarás); reproduction; habits.

Didelphis mudicaudatus, Desmarest (part not É. Geoffroy), 1827:390—myosuros Temminck a synonym. Waterhouse, 1841:94, Pl. 2 (animal). Jentink, 1888: 220 -part, syntypes of myosuros Temminck and opossum of Temminck collection. Thomas, 1888: 332-part, myosuros in synonymy.

Philander opossum frenasa [sic], Hershkovitz (part not Olfers), 1959:342-Didelphis quica Temminck in synonymy.

Metachirops opossum quichua [sic], Krumbiegel, 1941:200, 201, 206-name a reiterated lapsus for quica Temminck; BRASIL: São Paulo (Juquia, Serra do Mar).

TYPES-An unspecified number of skins, some mounted, skulls and skeletons of both sexes in the Vienna, Leiden, Paris, and Prince Maximilian zu Wied-Neuwied museums; female syntype in the Vienna Natural History Museum, collected 3 March 1818 by Johann Natterer, designated lectotype by Hershkovitz (1959, p. 337).

TYPE LOCALITY- "Bresil," restricted to "Sapitiba" (= Sepetiba), Rio de Janeiro (Rio de Janeiro, $\left.22^{\circ} 58^{\prime} \mathrm{S}, 43^{\circ} 42^{\prime} \mathrm{W}\right)$, by J. A. Allen ( 1900 , p. $195 ; 1916$ c, p. 562). 
TABLE 10. Measurements of Philander opossum quica. Means (extremes in parentheses) and number of specimens.

Locality Head and Body

Tail Hind Foot

Ear

Brasil oे oे

Anápolis

Trinidade

Urucum

Santa Teresa, ES

Campinho

Vitória

Vila Velha

Engenheiro Reeve

Juiz de Forna

Além Paraíba

Fazenda Cardoso

Teresópolis

Barro Branco

Macieiras

São João Marcos

Margaratiba

Boraceia

Piquete

Cotia

Butantã

São Sebastião

Iporanga

Roça Nova

Joinville

Hansa

Taquara

Brasil $q$ 우

$272(260-285) 5$

$295(280-305) 5$

$250,280,255$

215, 290, 297

270

275

240,255

265

290

273

263, 244

$228,252,296$

246

-

270

250

237 (210-260) 7

-

$-$

267

260

300

280, 280, 275

$245,195,355$

300

335

300,285

285

330

310

280, 291

$320,272,335$

346

-

330

320

$288(272-300) 7$

270

-

-

295

-

275

300

310

(a)

$42(38-45) 5$

$40,38,40$

$43,44,48$

40

42

38,39

32

42

29

40,39

$40,42,43$

44

$-$

45

39

$35(30-40) 7$

$-,-,-$

34,34 , -

30

32

28,30

30

30

38

38

$-,-,-$

22

-

30

- $31(30-33) 4$

- -

-

$-$

$\overline{35}$

35

$\overline{4} 1$

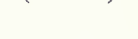

$-$

$-$

30

30

-

Seringal

Anápolis

Santa Teresa, MG

Santa Teresa, ES

Campinho

Faz. Floresta

Benfica

Além Paraíba

Tereópolis

Barro Branco

Marngaratiba

Pedra Branca

Boraceia

Piquete

Monte Alegre

Cotia

São Sebastião

Avanhandava

Roço Nova

Paraguay $\delta \delta^{*}$

Sapucay

Tacuati

245

$259(240-265) 7 \quad 269(235-295) 7$

230

262,260

265

250

251

$220,247,250$

255

231

$270,220,330$

$-$

$-,-,-$

$-$

$-$

$265,-$

251,293

230

286

289

$258(239-279) 8$

Sapucay

Argentina 000

Chaco

Bolivia 00

Hamacas

Warnes, 3 locations
280

$-, 305$

275

310

266

$300,300,-$

305

278

$240,280,300$

$-$

$-,-,-$

280 , -

265, 277

220

305

292

$292(277-304) 8$

$34(32-35) 8$

$34(32-36) 8$

35

$39(36-42) 7$

36

39,36

40

$\overline{36}$

$-, 34,40$

45

36

$34,32,35$

$-$

$-$

$-$

$36,-$

$-,-$

33

34

-

31,30

32

$-$

$32,31,29$

29

28

$26,22,31$

-, -, -

$-$

$31,-$

25,25

$\overline{32}$

35

36

32

$-$

297

$272(266-280) 5$

$39(37-41) 5$

$39(38-41) 5$ 


\begin{tabular}{|c|c|c|c|c|}
\hline $\begin{array}{l}\text { Condylobasal } \\
\text { Length }\end{array}$ & $\begin{array}{l}\text { Zygomatic } \\
\text { Breadth }\end{array}$ & Preorbital Width & Postorbital Width & Braincase Width \\
\hline $65.3(63.0-67.9) 5$ & $34.8(33.4-36.0) 5$ & $11.9(11.0-12.8) 3$ & $8.6(8.2-9.0) 5$ & $21.0(19.2-23.0) 5$ \\
\hline - & 34.0 & 13.8 & 8.8 & 18.8 \\
\hline $65.2,65.2,66.0$ & $35.4,35.6,36.1$ &.,,--- & $8.0,8.0,8.5$ & $21.5,21.6,21.1$ \\
\hline $72.2,72.4,74.1$ & $39.5,-, 42.8$ &.,-- 15.7 & $8.7,-, 8.8$ & $20.4,-, 21.4$ \\
\hline 70.4 & 39.0 & 14.9 & 8.6 & 22.8 \\
\hline 66.8 & - & - & - & - \\
\hline $69.1,64.0$ & 41.3 .33 .7 & 14.6. 15.0 & $8.5,8.8$ & $20.4,20.0$ \\
\hline 63.0 & 32.7 & 11.4 & 9.1 & 19.3 \\
\hline 66.5 & 36.3 & 13.4 & 8.5 & 19.6 \\
\hline 68.3 & 37.0 & 15.3 & 8.4 & 20.7 \\
\hline $64.8,67.1$ & $33.7,31.5$ &.-- & $8.6,8.5$ & $20.5,20.0$ \\
\hline $66.1,63.7,75.5$ & $33.9,35.7,42.3$ &.,,--- & $8.8,7.8,9.6$ &,,--- \\
\hline 65.9 & 35.5 & - & 9.0 & 19.5 \\
\hline 70.6 & 40.3 & - & 8.5 & 23.1 \\
\hline 76.4 & 43.7 & - & 8.4 & - \\
\hline 75.4 & 43.7 & - & 8.9 & - \\
\hline $63.7(60.0-68.7) 7$ & $33.1(30.7-37.8) 7$ & 11.4 & $8.4(7.9-9.0) 8$ & $19.3(18.9-20.6) 8$ \\
\hline 66.9 & 39.2 & - & 8.8 & 20.7 \\
\hline 66.0 & 36.0 & - & 9.0 & 21.1 \\
\hline 71.8 & 39.9 & _- & 8.6 & - \\
\hline 66.5 & 34.1 & 13.0 & 8.4 & 18.2 \\
\hline 68.0 & 38.8 & - & 8.1 & 21.3 \\
\hline 64.9 & 33.0 & 11.8 & 8.6 & 20.1 \\
\hline 72.6 & 40.3 & - & 8.6 & 22.2 \\
\hline 66.8 & 34.1 & 11.0 & 8.2 & 19.2 \\
\hline 71.8 & 36.6 & 12.9 & 7.9 & 20.3 \\
\hline 62.3 & 32.3 & 14.6 & 8.7 & 18.7 \\
\hline $64.1(61.0-65.7) 8$ & $34.0(32.6-35.7) 8$ & $11.5(11.0-12.3) 4$ & $8.6(8.4-8.7) 8$ & $20.9(19.7-21.7) 8$ \\
\hline 61.8 & 31.6 & 13.3 & 7.5 & 19.2 \\
\hline $64.7,68.0$ & $34.0,34.9$ & $14.2,14.9$ & $8.5,8.6$ & $18.3,18.5$ \\
\hline 67.0 & 35.4 & 14.7 & 8.2 & 18.8 \\
\hline 62.9 & 32.7 & - & 9.7 & 20.9 \\
\hline 59.0 & 31.1 & _- & 9.9 & 20.2 \\
\hline $58.8,63.4,65.5$ & $30.7,32.0,34.4$ & $14.7,14.2,-$ & $9.4,8.2,8.8$ & $20.9,18.1,18.5$ \\
\hline 63.3 & - & - & - & - \\
\hline 60.5 & _- & - & - & - \\
\hline $62.5,64.7,67.5$ &,,--- &,,--- &,,--- &,,--- \\
\hline 60.9 & - & - & - & - \\
\hline $58.9,62.2,60.8$ & $30.4,32.2,-$ &,,--- & $8.2,9.1,-$ & $19.8,19.9,-$ \\
\hline 62.8 & 31.0 & - & - & 19.2 \\
\hline 61.5 & 30.0 & - & 9.0 & 18.5 \\
\hline $63.4,62.2$ & $34.7,31.6$ &,-- & $8.9,8.6$ & $19.1,19.2$ \\
\hline $61.6,58.5$ & $31.1,29.4$ & $12.2,11.4$ & $8.4,8.7$ & $18.4,18.0$ \\
\hline 63.3 & 34.4 & - & 8.9 & 20.0 \\
\hline 57.1 & 28.0 & 9.7 & 8.2 & 18.5 \\
\hline 67.2 & 36.8 & 13.0 & 8.6 & 20.7 \\
\hline 64.6 & 34.7 & 12.6 & 8.6 & 19.3 \\
\hline $62.4(58.3-67.8) 9$ & $32.4(30.3-35.2) 9$ & $11.5(10.7-12.4) 9$ & $8.7(8.4-9.1) 9$ & $20.0(19.0-21.7) 9$ \\
\hline 69.0 & 39.2 & 13.0 & 9.0 & 19.8 \\
\hline 66.0 & 33.5 & - & 8.5 & 19.2 \\
\hline $61.8(60.5-62.6) 5$ & $33.0(31.8-34.6) 5$ & $11.3(10.8-11.8) 5$ & $7.7(7.4-8.4) 5$ & $19.7(19.0-20.1) 5$ \\
\hline
\end{tabular}


TABle 10. Continued.

\begin{tabular}{|c|c|c|c|c|}
\hline Locality & Head and Body & Tail & Hind Foot & Ear \\
\hline El Beni, 7 locations & $257(235-271) 5$ & $297(284-319) 5$ & $41(35-45) 5$ & $39(38-42) 5$ \\
\hline San Joaquín & 200 & 300 & 37 & 38 \\
\hline \multicolumn{5}{|l|}{ Bolivia 9 ? } \\
\hline Santa Cruz, 3 locations & $262,227,254$ & $242,305,295$ & $35,40,42$ & $41,43,41$ \\
\hline El Beni, 9 locations & $255(225-275) 10$ & $267(249-293) 10$ & $39(35-44) 10$ & $35(30-38) 10$ \\
\hline San Joaquín & - & - & - & - \\
\hline \multicolumn{5}{|l|}{ Perú ơ ठั } \\
\hline Cadena, Hda. & - & - & - & - \\
\hline Quincemil & 244 & 267 & 38 & 34 \\
\hline Urubamba, Río & - & - & - & - \\
\hline Balta & $248(232-255) 5$ & $278(253-299) 5$ & $42(39-44) 5$ & $35(31-36) 5$ \\
\hline Tingo María & 278 & 292 & 41 & 37 \\
\hline Pucallpa & 270,264 & 290,304 & 37,37 & 36,33 \\
\hline Yarinacocha & 291 & 286 & 48 & 38 \\
\hline Moyobamba & 275 & 293 & 45 & - \\
\hline Yurac Yacu & 257 & 292 & 43 & 33 \\
\hline \multicolumn{5}{|l|}{ Perú $q$ 운 } \\
\hline Maldonado, Pto. & 302 & 276 & 36 & 35 \\
\hline Boca Colorado & - & - & - & - \\
\hline Santa Rosa & - & - & - & - \\
\hline Urubamba, Río & - & - & - & - \\
\hline Chicosa & 241 & 263 & 36 & 32 \\
\hline Balta & 238 & 282 & 43 & 31 \\
\hline Cumaria & 240 & 280 & 38 & 34 \\
\hline Pucallpa & 273 & 297 & 40 & 34 \\
\hline Yarinacocha & 292 & 291 & 46 & 36 \\
\hline Sarayacu &,,--- &,,--- &,,--- &,,--- \\
\hline Apayacu & - & - & - & - \\
\hline \multicolumn{5}{|l|}{ Colombia $\delta ð$} \\
\hline Villavicencio & 265 & 270 & 40 & 32 \\
\hline Covaría, Río & 267 & - & 40 & 33 \\
\hline \multicolumn{5}{|l|}{ Colombia $ㅇ$} \\
\hline Villavicencio & - & - & - & - \\
\hline \multicolumn{5}{|l|}{ Venezuela $\delta \partial$} \\
\hline Motatán & 256 & 288 & 42 & 35 \\
\hline Río de Oro & 280 & 295 & 40 & 35 \\
\hline \multicolumn{5}{|l|}{ Venezuela 우우 } \\
\hline Motatán & 242,248 & 288,299 & 41,41 & 33,30 \\
\hline Yuruán, Río & 244,236 & 289,279 & 38,36 &,-- \\
\hline
\end{tabular}

Distribution-Map, Figure 5. PERÚ: Departments of Ucayali, San Martín, Huánuco, Cuzco, Ucayali, Madre de Dios; BOLIVIA: La Paz, El Beni, Cochabamba, Santa Cruz; PARAGUAY, ARGENTINA: Chaco, Misiones; BRASIL: Acre, Rondônia, Mato Grosso do Sul, Goias, Distrito Federal, Minas Gerais, Espírito Santo, Rio de Janeiro, São Paulo, Paraná, Santa Catarina, Rio Grande do Sul; altitudinal range sea level to about $1500 \mathrm{~m}$ above. See "Specimens Examined," p. 50 , for details.

Characters-Average body size smallest of the subspecies; underparts generally pale, sharply defined from sides (except some individuals in eumelanic color phase); tail usually parti-colored; molars relatively large, conule $C$ of $\mathrm{m}^{3}$ rudimentary. Individuals nurtured in captivity may be exceptionally large.

COMPARISONS-Philander opossum quica is the small, "gray," large-toothed form generally regarded as typical of the species. The Guianan $P$. opossum is larger, with more saturate pheomelanin, particularly on underparts. Philander opossum melanurus is slightly larger but always distin- 
TABLE 10. Extended (continued).

\begin{tabular}{|c|c|c|c|c|}
\hline $\begin{array}{l}\text { Condylobasal } \\
\text { Length }\end{array}$ & $\begin{array}{l}\text { Zygomatic } \\
\text { Breadth }\end{array}$ & Preorbital Width & Postorbital Width & Braincase Width \\
\hline $66.5(64.2-70.2) 7$ & $34.6(33.4-36.6) 7$ & - & $8.1(7.8-8.6) 7$ & $20.5(19.7-21.2) 7$ \\
\hline 69.3 & 38.2 & 11.6 & 8.3 & 21.3 \\
\hline $59.0,63.1,60.0$ & $31.2,33.2,31.4$ & $10.5,11.1,11.4$ & $8.4,8.2,8.0$ & $19.2,19.1,18.8$ \\
\hline $62.2(59.0-65.9) 9$ & $32.5(31.0-35.2) 9$ & $10.3(9.8-11.5) 6$ & $7.9(7.7-8.5) 9$ & $19.8(18.9-21.0) 9$ \\
\hline $61.3(59.8-62.4) 4$ & $32.8(31.5-34.0) 4$ & $10.4(10.2-10.8) 4$ & $7.8(7.7-8.1) 4$ & $19.4(18.3-20.0) 4$ \\
\hline 60.5 & 32.6 & - & 8.5 & 20.8 \\
\hline 62.0 & 31.9 & - & 8.2 & 20.5 \\
\hline 62.0 & - & - & 8.3 & - \\
\hline $65.0(62.5-67.5) 5$ & $33.3(31.7-34.3) 5$ & $12.0(11.8-12.3) 5$ & $8.5(8.0-8.8) 4$ & $19.1(18.4-19.6) 5$ \\
\hline 68.3 & 34.5 & 12.4 & 8.3 & 19.6 \\
\hline $65.1,64.2$ & $36.3,36.2$ & $11.9,12.3$ & $8.5,8.9$ & $20.9,21.4$ \\
\hline 71.5 & 40.8 & 14.8 & 8.8 & 20.4 \\
\hline 67.5 & - & - & 8.9 & 19.8 \\
\hline 64.8 & 32.9 & - & 8.3 & 19.7 \\
\hline 64.3 & 31.2 & 12.1 & 8.2 & 18.5 \\
\hline 63.4 & 31.9 & - & 8.6 & 20.2 \\
\hline 61.7 & - & - & - & - \\
\hline - & - & - & 8.3 & - \\
\hline 59.4 & 30.5 & 10.5 & 7.5 & 18.1 \\
\hline 67.5 & 33.0 & 12.3 & 7.8 & 18.6 \\
\hline 61.7 & 29.5 & 10.4 & 7.5 & 19.5 \\
\hline - & 33.5 & - & - & 19.9 \\
\hline 72.4 & 38.3 & 13.4 & 9.0 & 20.0 \\
\hline $66.9,68.0,71.9$ & $-, 34.0,35.9$ &,,--- & $8.6,8.4,9.0$ & $-, 20.2,22.0$ \\
\hline 63.5 & 33.9 & - & 9.3 & 21.9 \\
\hline 64.9 & 34.9 & - & 8.3 & 19.6 \\
\hline 65.1 & 33.6 & 12.0 & 8.4 & 20.8 \\
\hline 64.2 & 33.0 & - & 8.7 & 19.3 \\
\hline 64.6 & 34.6 & 11.9 & 8.4 & 20.5 \\
\hline 61.8 & 34.2 & - & 8.0 & 20.8 \\
\hline $60.8,63.4$ & $30.5,33.3$ & 10.6,- & $8.4,8.0$ & $20.0,19.9$ \\
\hline $58.5,61.2$ & $30.6,-$ &,-- & $8.2,-$ & $19.2,-$ \\
\hline
\end{tabular}

guishable by its chocolate-brown upper parts and saturate orange underparts.

MEASUREMENTS-Tables 1 and 10.

TAXONOMIC History-Didelphis quica was described by Temminck from several mounted skins and skeletons in the Leiden Museum and from other specimens in the Vienna, Paris, and Prince Maximilian zu Wied-Neuwied museums. Four live and several spirit-preserved individuals are also mentioned as having been seen. In his catalog of the Leiden Museum collection, Jentink (1888, p. 220) lists both the mounted skin of an adult female as "un des types de l'espéce. Bresil. Des collections de M. Natterer. Didelphis larvata" and a mounted subadult male as "un des type l'espéce. Bresil. Du Cabinet de M. Temminck. Didelphis crassicaudata Desmarest."

Temminck (1824, p. 37) noted particularly that best knowledge of the species is based on "quicas" shipped to the Vienna Museum by Johann Natterer. This information prompted J. A. Allen (1900, p. 195; 1916c, p. 562) to restrict the type locality of Temminck's quica to Sepetiba, Rio de 
Table 10. Continued.

\begin{tabular}{|c|c|c|c|}
\hline Locality & Palatal Length & $\mathrm{i}-\mathrm{m}^{4}$ & $\mathbf{m}^{1-4}$ \\
\hline \multicolumn{4}{|l|}{ Brasil రే రే } \\
\hline Anápolis & $39.7(38.5-41.7) 5$ & $34.4(34.0-35.4) 5$ & $13.1(12.8-13.3) 5$ \\
\hline Trinidade & 37.8 & 33.8 & 12.9 \\
\hline Urucum & $35.8,38.7,38.9$ & $33.8,32.8,34.2$ & $12.7,12.6,12.6$ \\
\hline Santa Teresa, ES & $41.1,-, 42.0$ & $35.5,-, 35.0$ & $12.4,12.4,12.5$ \\
\hline Campinho & - & 35.8 & 12.1 \\
\hline Vitória & - & - & 12.5 \\
\hline Vila Velha &,-- & $35.5,34.4$ & $12.2,12.2$ \\
\hline Engenheiro Reeve & 37.4 & 33.2 & 11.6 \\
\hline Juiz de Forna & 39.8 & 34.3 & 12.3 \\
\hline Além Paraíba & 40.5 & 34.9 & 12.4 \\
\hline Fazenda Cardoso & $38.8,34.1$ & $33.6,30.9$ & $12.3,11.5$ \\
\hline Teresópolis & $39.0,39.4,44.8$ & $33.5,34.0,37.4$ & $12.0,12.1,12.6$ \\
\hline Barro Branco & 38.5 & 33.5 & 12.1 \\
\hline Macieiras & 41.0 & 33.9 & 12.0 \\
\hline São João Marcos & 43.3 & 35.9 & 12.5 \\
\hline Mangaratiba & 43.4 & 36.6 & 12.7 \\
\hline Boraceia & $37.8(35.6-40.1) 8$ & $33.3(32.0-34.3) 8$ & $12.2(11.9-13.0) 8$ \\
\hline Piquete & 39.3 & 33.9 & 12.7 \\
\hline Cotia & 39.1 & 34.6 & 12.3 \\
\hline Butantã & 42.9 & 36.6 & 12.2 \\
\hline São Sebastião & 39.4 & 34.5 & 12.0 \\
\hline Iporanga & 39.8 & 34.0 & 12.2 \\
\hline Roça Nova & 39.3 & 34.1 & 12.3 \\
\hline Joinville & 41.7 & 34.9 & 11.8 \\
\hline Hansa & 40.3 & 34.8 & 12.2 \\
\hline Taquara & 43.1 & 37.1 & 12.8 \\
\hline \multicolumn{4}{|l|}{ Brasil $q 9$} \\
\hline Seringal & 38.8 & 33.6 & 13.2 \\
\hline Anápolis & $38.9(38.5-40.7) 8$ & $34.0(33.0-34.8) 8$ & $13.0(12.6-13.5) 8$ \\
\hline Santa Teresa, MG & 36.4 & 32.9 & 12.3 \\
\hline Santa Teresa, ES & $38.2,40.9$ & $33.0,34.5$ & $12.3,12.5$ \\
\hline Campinho & 39.0 & 34.1 & 12.0 \\
\hline Faz. Floresta & 37.5 & 33.0 & 11.9 \\
\hline Benfica & 35.3 & 31.0 & 11.8 \\
\hline Além Paraíba & $35.5,37.5,40.0$ & $32.0,33.6,34.0$ & $12.0,12.1,12.0$ \\
\hline Teresópolis & 37.1 & 32.1 & 11.9 \\
\hline Barro Branco & - & - & 12.5 \\
\hline Mangaratiba &,,--- &,,--- & $11.6,12.3,11.9$ \\
\hline Pedra Branca & - & - & 12.0 \\
\hline Boraceia & $35.6,37.8,-$ & $30.5,32.0,-$ & $11.4,11.5,11.2$ \\
\hline Piquete & 37.6 & 33.3 & 12.0 \\
\hline Monte Alegre & 36.7 & 32.8 & 11.8 \\
\hline Cotia & $37.8,37.1$ & $33.1,32.0$ & $11.5,11.5$ \\
\hline São Sebastião & $36.6,35.1$ & $32.6,32.0$ & $11.8,11.9$ \\
\hline Avanhandava & 37.4 & 32.5 & 11.8 \\
\hline Roço Nova & 35.2 & 31.8 & 12.2 \\
\hline \multicolumn{4}{|l|}{ Paraguay ot 0} \\
\hline Sapucay & 40.7 & 34.9 & 12.6 \\
\hline Tacuati & 38.0 & 33.4 & 12.3 \\
\hline \multicolumn{4}{|l|}{ Paraguay $q$ 의 } \\
\hline Sapucay & $37.3(35.1-39.0) 9$ & $32.8(31.5-33.8) 9$ & $12.2(11.9-12.8) 9$ \\
\hline \multicolumn{4}{|l|}{ Argentina $\delta \vec{\partial}$} \\
\hline Chaco & 41.8 & 36.1 & 13.5 \\
\hline \multicolumn{4}{|l|}{ Bolivia of $\delta$} \\
\hline Hamacas & 39.4 & 34.2 & 13.0 \\
\hline Warnes, 3 locations & $37.2(36.2-38.0) 5$ & $32.9(31.5-33.5) 5$ & $12.5(12.0-12.8) 5$ \\
\hline
\end{tabular}


TABle 10. Continued.

\begin{tabular}{|c|c|c|c|}
\hline Locality & Palatal Length & $i-m^{4}$ & $\mathbf{m}^{1-4}$ \\
\hline $\begin{array}{l}\text { El Beni, } 7 \text { locations } \\
\text { San Joaquín }\end{array}$ & $\begin{array}{l}39.9(39.1-41.7) 7 \\
38.0\end{array}$ & $\begin{array}{l}34.9(34.2-36.2) 7 \\
33.7\end{array}$ & $\begin{array}{l}13.3(12.6-13.7) 7 \\
12.8\end{array}$ \\
\hline Bolivia $\&$ \& & & & \\
\hline $\begin{array}{l}\text { Santa Cruz, } 3 \text { locations } \\
\text { El Beni, } 9 \text { locations } \\
\text { San Joaquín }\end{array}$ & $\begin{array}{l}36.0,39.0,37.2 \\
37.8(35.4-39.8) 9 \\
37.2(36.6-37.7) 4\end{array}$ & $\begin{array}{l}32.4,33.6,34.0 \\
33.3(32.0-35.3) 9 \\
32.7(32.2-33.2) 4\end{array}$ & $\begin{array}{l}12.5,12.4,13.0 \\
12.7(12.0-13.4) 9 \\
12.2(11.9-12.5) 4\end{array}$ \\
\hline $\begin{array}{l}\text { Perú } \delta \delta^{\star} \\
\text { Cadena, Hda. } \\
\text { Quincemil } \\
\text { Urubamba, Río } \\
\text { Balta } \\
\text { Tingo María } \\
\text { Pucallpa } \\
\text { Yarinacocha } \\
\text { Moyobamba } \\
\text { Yurac Yacu }\end{array}$ & $\begin{array}{l}36.6 \\
38.6 \\
38.2 \\
40.0(38.0-41.8) 5 \\
41.8 \\
39.8,39.9 \\
43.1 \\
40.6 \\
39.8\end{array}$ & $\begin{array}{l}32.9 \\
34.9 \\
33.7 \\
35.3(34.0-36.9) 5 \\
36.5 \\
34.0 .32 .5 \\
37.7 \\
35.7 \\
34.7\end{array}$ & $\begin{array}{l}12.5 \\
13.6 \\
12.5 \\
13.5(12.9-14.3) 5 \\
13.2 \\
13.0,12.5 \\
14.4 \\
13.4 \\
14.2\end{array}$ \\
\hline $\begin{array}{l}\text { Perú } 9 \text { \& } \\
\text { Maldonado, Pto. } \\
\text { Boca Colorado } \\
\text { Santa Rosa } \\
\text { Urubamba. Río } \\
\text { Chicosa } \\
\text { Balta } \\
\text { Cumaria } \\
\text { Pucallpa } \\
\text { Yarinacocha } \\
\text { Sarayacu } \\
\text { Apayacu }\end{array}$ & $\begin{array}{l}39.8 \\
39.1 \\
37.8 \\
36.3 \\
36.5 \\
41.3 \\
38.5 \\
- \\
45.7 \\
42.3,42.4,44.6 \\
39.8\end{array}$ & $\begin{array}{l}33.8 \\
33.8 \\
33.8 \\
31.8 \\
32.2 \\
37.3 \\
33.6 \\
- \\
38.5 \\
38.0,36.8,37.6 \\
34.8\end{array}$ & $\begin{array}{l}12.6 \\
13.1 \\
12.7 \\
12.3 \\
12.5 \\
14.5 \\
13.1 \\
13.1 \\
14.0 \\
13.4,13.6,13.5 \\
12.2\end{array}$ \\
\hline $\begin{array}{c}\text { Colombia ơ o } \\
\text { Villavicencio } \\
\text { Covaría, Río }\end{array}$ & $\begin{array}{l}38.6 \\
39.3\end{array}$ & $\begin{array}{l}33.7 \\
34.1\end{array}$ & $\begin{array}{l}13.1 \\
13.1\end{array}$ \\
\hline $\begin{array}{l}\text { Colombia } \uparrow ? \\
\text { Villavicencio }\end{array}$ & 39.0 & 33.4 & 12.3 \\
\hline $\begin{array}{l}\text { Venezuela ơ ơ } \\
\text { Motatán } \\
\text { Río de Oro }\end{array}$ & $\begin{array}{l}40.0 \\
38.0\end{array}$ & $\begin{array}{l}34.1 \\
34.0\end{array}$ & $\begin{array}{l}13.1 \\
13.5\end{array}$ \\
\hline $\begin{array}{c}\text { Venezuela } 9 \text { } 9 \\
\text { Motatán } \\
\text { Yuruán, Río }\end{array}$ & $\begin{array}{l}32.5,38.8 \\
35.5,37.2\end{array}$ & $\begin{array}{l}33.0,33.7 \\
32.5,32.4\end{array}$ & $\begin{array}{l}12.9,12.6 \\
12.9,12.4\end{array}$ \\
\hline
\end{tabular}

Janeiro. Pelzeln (1883, p. 110) mentioned Sepetiba as one of four localities where Natterer collected a total of 15 specimens, including one live, all sent to Vienna. Pelzeln (loc. cit.) added the descriptions of an old female collected 3 March 1818 and a male collected 29 March 1818, both from Sepetiba. The female has since been designated the lectotype.

Didelphis myosuros Temminck is based on one or more pouched adult female four-eyed opossums and an unspecified number of pouched "jeunes femelles [qui] nous parviennant en peaux des- seches." Males are not mentioned in the description proper, but Temminck (1825, p. 40) stated he examined individuals of both sexes in the PaysBas (Leiden) Museum. He might have seen more in the "Musées de Vienna, de Francfort et du prince de Neuwied." In any event, certain aspects of the description may not have been based solely on "les femelles [qui] ont une poche complete." For example, the tail base is described as furred a distance of 10 lines (about $2 \mathrm{~cm}$ ) in two adults and nine lines in a subadult. This is characteristic of nonpouched Metachirus mudicaudatus. On the 
other hand, the nearly equal body and tail proportions of the same individuals and unpigmented tail base indicate the pouched species. The descriptions of external characters lack precision, but some details of the original text apply equally well to both pouched and nonpouched species but in no case to the latter alone.

Despite the apparent composite nature of the description, Temminck (1824, p. 39) denied that his myosure might be identical with $D$. nudicaudata É. Geoffroy, because, he notes, "il est dit dans le texte que la queue de cet animal est toute nue, et que la femelle n'a point de poche." Temminck (1824, p. 40) drew attention, nevertheless, to a poorly preserved skin of a young female in the Paris museum labeled $D$. nudicaudata that, he said, agreed in every respect with $D$. myosuros. $\mathrm{He}$ questioned, however, that this individual may have served É. Geoffroy for the original description of $D$. nudicaudata. Notwithstanding, authors beginning with Wied-Neuwied (1826) and Desmarest $(1827$, p. 390$)$ concluded that Temminck did indeed identify his $D$. myosuros with $D$. nudicaudata. The mistaken identity gave rise to confusion in later years.

Wied-Neuwied (1826, p. 400, Pl. 2, Fig. 5 [skull]) described one of two male four-eyed opossums captured in "Comechatiba" (= Comoxatibá or Cumuraxatibá, $17^{\circ} 06^{\prime} \mathrm{S}, 39^{\circ} 11^{\prime} \mathrm{W}$ ), between the Rios Prado and Corumbão, Bahia, Brasil, and referred them to D. myosuros Temminck. The finely detailed characterizations and the figured skull of the animal are unquestionably those of Metachirus nudicaudatus. Wied-Neuwied, however, could not be certain of the propriety of the name myosuros because he (1826, p. 405) "had not seen a female which, according to Temminck, has a pouch" (my translation of the original German).

In his supplement to Schreber's Säugethiere, Wagner (1843, p. 43) included Didelphis myosuros Temminck, emended to "Didelphis myosurus," in his fully pouched opossum group and short-haired subgroup (Wagner, 1843, p. 37"mastotheca ventrali ampla"; p. 42-"vellere brevi"). His description of D. myosurus, however, is based on the Berlin Museum's type specimen of Didelphis frenata Lichtenstein (Olfers): Didelphis nudicaudata É. Geoffroy was listed as a synonym of Didelphis myosurus without explanation.

Waterhouse (1846, p. 482) treated Didelphis myosuros Temminck, 1824, as a junior synonym of Didelphis nudicaudata É. Geoffroy, 1803. His description, based on a specimen in the British
Museum, is unquestionably that of the pouchless species. Evidently females were not seen, and Waterhouse mistakenly included Didelphis nudicaudata along with $D$. philander (= Caluromys philander) in his Didelphis "Section 1. Opossums in which the pouch is well developed."

In his account of the mammals of Rio de Janeiro and Minas Gerais, Burmeister (1854, p. 135) accepted Wagner's (1843) use of the emended form of the name Didelphis myosurus Temminck and treatment of $D$. nudicaudata as a synonym. Burmeister saw no specimens of myosuros in southeastern Brasil, and he drew on Wied-Neuwied (1826) for a description of the species. Shortly thereafter, Burmeister (1856, p. 68, Pl. 10) described a male and female labeled "Didelphis myosurus Temminck" in the Berlin Museum. His characterization of the two animals, believed to have originated in Pará, is somewhat equivocal but fits that of Didelphis nudicaudata É. Geoffroy, cited as a synonym. The adult male illustrated in color is identifiable as Metachirus nudicaudatus.

Thomas (1888) cited "Didelphis myosurus, Temm." and its usage by other authors, including Wied-Neuwied (1826), Wagner (1843), and Burmeister $(1854 ; 1856)$, in the synonymy of " $\mathrm{Di}$ delphys nudicaıdata É. Geoffroy."

Jentink (1888, pp. 220, 221) listed the following skins identified as Didelphis nudicaudata É. Geoffroy in his catalog of mammals of the Muséum d'Histoire Naturelle des Pays-Bas, where many of Temminck's specimens are deposited:

a. Individu adulte monté, un des types du Didelphis myosuros Temminck. Brésil.

b. Male adulte monté, un des types du Didelphis myosuros Temminck. Brésil, 1822.

c. Male adulte monté. Brésil.

d. Male semi-adulte monté. Brésil. Du Cabinet de M. Temminck. Didelphis opossum.

It is unlikely that most, if any, of the above male and unsexed "types" of D. myosuros actually served as bases for the original description of the species. The females of the "plusieurs individus des deux sexes" mentioned by Temminck (1825, p. 40) are missing. Individual a, listed by Jentink as a "type," may be a specimen subsequently labeled "myosuros" by Temminck. Specimen b, dated 1822, is certainly one of those collected by Natterer the same year in Registo do Sai (April) or Ypanema (January, June) and sent to the Vienna Museum (Pelzeln, 1883, p. 111). These Natterer specimens represent the opossum 
sometimes incorrectly known as $M$. nudicaudatus personatus Miranda Ribeiro.

Misapplication of the name Didelphis myosuros Temminck for pouchless four-eyed opossums was pointed out in 1959 (Hershkovitz, 1959, p. 343), and treatment of the name as a junior synonym of Philander opossum frenata [sic] Olfers was suggested. On the other hand, my designation of the Comoxatiba opossum recorded by Wied-Neuwied as lectotype of myosuros Temminck is invalid. The specimen, a male Metachirus mudicandatus, was not mentioned by Temminck in his description of myosuros and likely was not seen by him at any time.

It is now proposed to designate as lectotype of myosuros one of the pouched females used by Temminck in the original description of the taxon. In the absence of evidence to the contrary, the type locality, said to be Brasil, is here restricted to southeastern Brasil. Thus, myosuros (= myosurus) Temminck becomes a synonym of $P$. opossum quica Temminck.

Metachirus canus Osgood (1913), from Moyobamba. Perú, based on a single specimen, was distinguished from western Colombian grisescens (=P. opossum melanurus) by its generally paler coloration and parti-colored tail. Comparisons were not made with $P$. opossum quica, from which it is indistinguishable, presumably for lack of material at hand from Bolivia, Paraguay, and southeastern Brasil.

The Paraguayan Philander opossum azaricus Thomas (1923, pp. 604) was characterized as a "uniform grey subspecies without blackened crown." Thomas' (1923, p. 604) description of Philander opossum crucialis from Santa Cruz de la Sierra, Bolivia, is virtually a paraphrase of that of azaricus. The type, a "remade skin," "somewhat faded," was collected about a half-century earlier by Thomas Bridges. Later, with "good series" of the Peruvian canus and Bolivian crucialis before him, Thomas (1928b, p. 294) opined that the "two forms should not be considered as distinct from each other." Krumbiegel (1941, p. 204) regarded azaricus and crucialis indistinguishable.

Except for the color phases, seasonal differences in pelage, and certain exceptionally large individuals, I find a remarkably high degree of uniformity in the morphology of the common Philander opossums of eastern Brasil, northern Argentina, Paraguay, lowland Bolivia, eastern Perú, and Ecuador. Material from Colombia and western Venezuela is similar, but the disjunct distribution suggests convergence.
VARIATION-Material from Rio de Janeiro includes individuals of $P$. opossum quica with saturate coats like those of $P$. opossum opossum from Suriname and others with the typical gray coat. A large series (27) from Anápolis, Goias, appears to be intermediate between $P$. opossum opossum and the gray, eastern Brasilian P. opossum quica. Seven adults in old pelage, taken October through February, appear brown because of exposure of the basal portion of the dorsal hairs. Eight adults in fresh winter pelage (April-August) appear grayish.

In the more southern states of eastern Brasil, and in Paraguay, Bolivia, and Perú, the animals are predominantly grayish or grayish brown dorsally, the underparts pale.

Dichromatism in nine specimens of quica from Balta, Ucayali, Perú, parallels that of $P$. andersoni mcilhennyi from the same locality. The color difference, however, is not sexual. In six specimens (two $q q$, four $\delta^{\circ} \delta^{*}$ ), underparts are pheomelanic, the hairs buffy or orange to the roots, as in female $P$. andersoni. In three specimens ( $q$, $\left.\delta^{\circ}\right)$, underparts are dominantly eumelanic or grayish, the hairs pheomelanic terminally, eumelanic basally, and showing through at the surface, as in male $P$. andersomi. The tail of the single female of the eumelanic series is entirely pigmented. The terminal whitish portion in all other members of the Balta series varies from about one-third to oneeighth of total length without significant difference otherwise between those of the two color phases. Dorsum and sides are likewise nearly the same in both, but crown and muzzle are more deeply and extensively brown in the eumelanic or gray-phase group.

Convergent dichromatism between the Balta $P$. opossum and $P$. andersoni throws more doubt on the validity of the concept of "character displacement" in mammals.

An extremely large male and a female from Yarinacocha, Río Ucayali, Perú, agree with $P$. andersoni mcilhennyi with respect to body size and dark underparts and limbs but lack the diagnostic dark dorsal band. They are readily distinguished by dorsal coloration and pelage pattern from the intermediate series of quica taken at nearby $\mathrm{Pu}$ callpa as well as by absence of sexual dichromatism. They also differ from Pucallpa $P$. opossum quica by larger size and darker color throughout but agree in dental and cranial characters. The pigmentation of underparts and limbs is interpreted as the dark-phase coloration of occasional occurrence in $P$. opossum quica. The extremely 
large size of each of the Yarinacocha samples may be attributed to extreme old age and possibly unusually favorable living conditions comparable to those of the captive quica recorded from eastern Brasil (see above, p. 11).

The opossums from the Río Orinoco basin in Colombia and Venezuela are practically indistinguishable from Perúvian $P$. opossum quica or from the Field Museum material from Bolivia and Brasil. They may represent a case of parallelism or may be a disjunct remnant of an erstwhile widely distributed subspecies $P$. opossum quica which may not have differentiated much if anything from the hypothetical ancestral form of the species.

While this monograph was in preparation, Patton et al. (1995 [1996]) had the opportunity to examine tissues for molecular studies of one specimen of $P$. opossum frenatus from "Brasil; Rio de Janeiro; Najé, Carafão, Est. Teresópolis.” Fifteen specimens from Teresópolis in the $\mathrm{BM}[\mathrm{NH}]$, MNRJ, and USNM I examined were identified on the basis of skins and skulls as $P$. opossum quica. Present knowledge of $P$. opossum frenatus does not permit its synonymy with any other known four-eyed opossum.

According to Patton et al. (1995 [1996], pp. 21, 22), $P$. opossum frenatus "is quite divergent from $P$. mcilhennyi and $P$. o. canus $[=P$. o. quica $]$ (two taxa that are sympatric over much of western Amazonia in Brasil and Perú, with an average divergence of $16.45 \%)$. Consequently, frenatus and (opossum canus + mcilhennyi) form a basal trichotomy with the three species of Didelphis in all analyses."

There appears to be a contradiction between the 15 Teresópolis specimens I identified as $P$. opossum quica on geographic and morphological grounds and the Teresópolis tissue Patton finds unusually different from the others and the 426 specimens I examined over the range of $P$. opossum quica (Fig. 5). Perhaps the Patton et al. specimen is not quica, but it has not been shown to be frenatus.

SPecimens Examined-415. ARGENTINA. Chaco: Río Chico, 1 (Bm[NH]). BOLIVIA. El Beni: Arruda, I (FMNH); Barranquita, 1 (FMNH); Camiaco, 1 (AMNH); Camino Vilches, 1 (USNM); Centinela, I (FMNH); El Carmen, 1 (FMNH); Exaltación, 2 (AMNH; FMNH); Guayaramarin, 4 (AMNH); Itonama, 1 (USNM); Magdalena, 5 (FMNH); Mercedes, 1 (AMNH); Palácios, Río Mamoré, 4 km SE, 1 (AMnh); Puerto Caballo, 2 (AMNh); Puerto Siles, 2 (AMNH); Río lbaré, mouth, 4 (AMNH); Río Ma- moré, 12²6'S, 3 (AMNH; FMNH, 2); San Ignacio de Moxos, 1 (USNM); San Joaquín, 13 (FMNH); San Pablo, 1 (FMNH); San Ramón, 1 (FMNH); Santa Rosa, 1 (FMNH); Santo Dios, I (FMNH); Vaca Diez, Riberalto, 1 (USNM); Cochabamba: Río Ichilo, 52 km S, mouth, Río Chaparé, I (AMNH); La Paz: Chulumani, 1 (BM[NH]); Santa Cruz: Ascensión de Guarayos, 2 (FMNH); Buena Vista, 12 (AMNH; BM[NH], 8; FMNH, 3); El Palmar, 2 (USNM); Hamacas, 2 (AMNH); Ibañez, Río Piray, 2 (USNM); Río Chaparé, $2 \mathrm{~km} \mathrm{~S}$, mouth, I (AMNH); Santa Cruz, 3 (BM[NH], holotype of crucialis Thomas; USNM, 2); Santa Rosita, 4 (USNM); Tocomechi, Warnes, 1 (USNM); Warnes, 3 (USNM). BRASIL. Acre: Seringal Oriente, 4 (MPEG, 3; MZUSP); Espírito Santo: Cachoeira Bonita, Parque Nacional do Caparaó, 1 (MNR); Campinha, 4 (MNRJ); Engenheiro Reeve, 3 (BM[NH]); Pedra Roxa, Parque Nacional do Caparaó, 1 (MNRJ); Santa Teresa, 7 (MNRJ, 5; MZUSP, 2); Sierra, 3 (MNRJ); Vale Verde, Parque Nacional do Caparaó, 8 (MNRJ); Vila Velha, Morro de Angoles, 3 (MNRJ); Vitória, 2 (MNRJ); Goiás: Anápolis, 27 (AMNH); Aragarças, 2 (MPEG); Trinidade, 1 (MPEG); Mato Grosso: Cáceres, 1 (USNM); Monte Alegre, 5 (MZUSP); Santa Teresa, 1 (USNM); Urucum, 5 (AMNH); Minas Gerais: Alem Paraiba, 4 (MNRJ); Benfica, Serra de Itatiaia, 1 (AMNH); Bõa Esperança, Serra de Caparão, 3 (AMNH); Conceição de Mato Dentro, Boca de Ulaba, 1 (MNRJ); Fazenda Cardosa, Serra de Caparão, 2 (AMNH); Fazenda de Floresta, Rio Matipó, 2 (MZUSP); Fazenda S. Francisco de Caparão, 1 (BM[NH]); Juiz de Fora, 2 (MNRJ); Quartel de Sacramento, 1 (MZuSP); "S. Francisco de C.," 1 ( $\mathrm{Bm}[\mathrm{NH}]) ; \mathrm{Pa}$ raná: Rio Paracai, 1 ( $\mathrm{BM}[\mathrm{NH}])$; Roça Nova, Serra do Mar, 3 (вм[Nн]); Rio Grande do Sul: no precise locality, 1 ( $\mathrm{BM}[\mathrm{NH}])$; Rio de Janeiro: Barro Branco, 2 (MNRJ); Itatiaia, Maceira, 2 (MNRJ); Itatiaia, Parque Nacional, I (MNRJ); Mangaratiba, 5 (MNRJ); Pedra Branca, Paratí, 4 (MNRJ); Rio de Janeiro, 2 (AMNH); Rodico, Serra do Mar, 1 (BM[NH]); São João Marcos, 2 (MNRJ); Teresópolis, 15 (BM[NH]; MNRJ, 13; USNM); Rondônia: Porto Velho, I (USNM); Santa Catarina: Hansa, 4 (BM[NH]); Joinville, I (FMNH); São Paulo: Alto da Serra, 1 (MZUSP); Boraçeia, 26 (MZUSP, 25; USNM, 1); Butantã, Serra da Cantareira, 3 (MZuSP); Casa Grande, 3 (MZUSP); Cobia, 3 (MZUSP); Costão dos Engenhos, 1 (MZUSP); Iporanga, 5 (MNRJ); Lageado de Iporanga, 1 (MZUSP); Lageado do Aranhadavá, 1 (MZUSP); Monte Alegre, Amparo, 3 (MZUSP); Piquete, 2 (MZUSP); São Sebastião, 4 (BM[NH]); Serra de Itatiaia, 1 (MZUSP); Vila Oliveira, Mogi das Cruzes, 1 (MzusP). PERÚ. Cuzco: Hacienda Cad- 
ena, 2 (FMNH); Quincemil, 3 (FMNH); Huánuco: Tingo Maria, 2 (BM[NH]); Ucayali: Apayacu, 1 (AMNH): Balta, Río Curanja, 9 (Lsumz); Chicosa, 5 (вм[NH]); Cumaria, 1 ( $\mathrm{BM}[\mathrm{NH}])$; Iquitos, 1 (AMNH); Itaya, Iquitos, I (AMNH); Lagarto Cocha, 2 (AMNH); Pucallpa, 3 (USNM); Río Urubamba, mouth, 5 (AMNH); San Jerónimo, I (BM[NH]); Santa Rosa, 1 (AMNH); Sarayacu, 8 (AMNH); Yarinacocha, 4 (FMnH; LSUMz, 3); Madre de Dios: Boca Colorado, 1 (FMNH); Río Tambo, I (USNM); San Martín: Moyobamba, 3 (BM[NH], 2; FMNH); Rioja, 1 (BM[NH]); Yurac Yacu, 10 (BM[NH]). PARAGUAY. Sapucay, 21 (BM[NH], 8, including holotype of azaricus; USNM, 13); Tacuati, I (USNM).

\section{Philander opossum frenatus Olfers}

$D$ [idelphys]. frenata Illiger, 1815 (1804-1811):107nomen nudum.

$D$ [idelphys]. frenata Olfers, 1818:204

[Metachirops] frenata, Matschie, 1916:268-BRASIL: Bahia (type locality).

Met[achirops]. opossum frenatus, Krumbiegel, 1941: 206 (in (ext)-explicitly omitted from taxonomic review.

Philander opossum frenata [sic], Hershkovitz, 1959: 338, 342-taxonomy; part, synonyms (superciliaris Olfers, myosuros Temminck).

$D$ [idelphys]. superciliaris Illiger, 1815(1804-1811): 107-nomen nudum. Olfers, 1818:204-no locality.

$D$ [idelphis]. myosums [sic], Wagner, 1843, footnote 25, p. 44-BRASIL: Bahia; type of "frenata Lichtenstein" from Bahia; collected by Kaehne.

Metachirus quica, Burmeister (part, not Temminck), 1856:70, PI. 8 (female)-BRASIL: Bahia; type of Didelphys frenata llliger [Olfers] in Berlin Museum, figured.

Metachirus nudicandatus nudicaudatus, Cabrera (part, not É. Geoffroy, 1803), 1958:48-" frenata Lichtenstein (Wagner)" in synonymy.

TyPE-Adult female, skin mounted, Berlin Museum: collected before 1815 by Herr Kaehne.

TYPE LOCALITY - Originally given as Brasil; according to Wagner (1843, footnote 25, p. 44$)$ the specimen labeled $D$. frenata in the Berlin Museum was collected by Herr Kaehne in Bahia, presumably in the present São Salvador, which may be taken as the restricted type locality (cf. Matschie, 1916, p. 268).

Distribution (Fig. 5)-Known only from the ascribed type locality.

Characters-Olfers' (1818, p. 204) description of frenata (and superciliaris) reproduced below is little more than the indication required to validate the specimen label names first published as nomina nuda by Illiger.
"D[idelphys]. Opossum L[innaeus]

"Sarigoy, Sarigue Var.

"Carigueia Marc. 222. Piso 323 (excl. ic.)

“D. frenata und supercilia III. gehören

als varietaten hieher. Das Mannchen

hat Gelbe Flecken, das Weibchen

grössere Weisse über den Augen."

REMARKS-A pouched four-eyed opossum perhaps representative of $P$. opossum quica, may well have ranged into the erstwhile coastal forests of southern Bahia and possibly farther north. Pending examination of the type or specimens from Bahia, if any, the name frenatus is retained.

The specimens identified as $P$. opossum frenatus by Patton et al. (1995 [1996], pp. 7, 21, 22) are from Teresópolis in the state of Rio de Janeiro and well within the range of $P$. opossum quica. It has not been shown that the Teresópolis specimen is not $P$. opossum quica nor that it is representative of $P$. opossum frenatus.

\section{Philander opossum melanurus Thomas}

[?]Didelphis opossum, Thomas, 1888:329-part, ECUADOR: Guayas (Guayaquil).

Metachirus opossum melanurus Thomas, 1899:285type description. Lönnberg, 1913:36-ECUADOR: Pichincha (Gualea).

[Metachirops] melanurus, Matschie, 1916:268—classification.

[Holothylax opossum] melamurus, Cabrera, 1919:48classification.

Metachirops opossum melanurus, Lönnberg, 1921: 68-ECUADOR: Pichincha (Gualea).

Philander opossum melamurus, Cabrera, 1958:35part, not melantho (= fuscogrisens) in synonymy.

$P[$ hilander]. o[possum]. melanurus, Baker, 1974: 135-ECUADOR: Esmeraldas (Quinindé).

TYPE-Old male, skin and skull, British Museum (Natural History), no. 1897.11.7.61; collected 11 April 1897 by W. F. H. Rosenberg.

TYPE LOCALITY-Paramba, Río Mira, Imbabura, western Ecuador; altitude, $1100 \mathrm{~m}$.

Distribution (Fig. 5)-The humid forests of northwestern Ecuador in the provinces of Esmeraldas, eastern Manabí, and bordering parts of western Imbabura and Pichincha. Nothing is known of the opossums in the Colombian departments of Nariño and Cauca, where fuscogriseus and melanurus may intergrade or prove to be sympatric.

Characters-Dorsum dominantly brownish agouti, underparts buffy to deep orange often with dark basal portions of hair showing through; tail brown but extreme tip sometimes whitish. 
TABLE 11. Measurements of Philander opossum melanurus. Means (extremes in parentheses) and number of specimens.

\begin{tabular}{cllll}
\hline Locality & Head and Body & \multicolumn{1}{c}{ Tail } & Hind Foot & Ear \\
\hline Ecuador o o & & & & \\
Mindo, below & - & - & - & - \\
Paramba & $269(258-283) 5$ & $262(250-276) 5$ & $41(40-43) 5$ & $30(27-35) 5$ \\
San Javier & 260 & 270 & 38 & 28 \\
Carondelet & 296 & 269 & & \\
Ecuador 9 ? & & & 35 & 28 \\
Río Caoní & 284 & 270 & 37 & 32 \\
San Javier & 282 & 229 & & \\
\hline
\end{tabular}

COMPARISONS-Dark agouti upper parts, heavily pigmented pheomelanic underparts mixed with gray, and entirely or almost entirely brown tail distinguish melanurus from nearly all fuscogriseus and quica; size averages less than fuscogriseus, greater than quica.

Measurements-Tables 1 and 11 .

REMARKS-Comparatively small size, dark agouti upperparts and dark tail are the most distinctive characters of $P$. opossum melanurus; distinction from fuscogriseus is doubtful.

SPECIMENS EXAmINED-15. ECUADOR. Esmeraldas: Carondelet, 1 (USNM); Esmeraldas, 1 (AMNH); San Javier, 5 (USNM); Imbabura: Paramba, 6 (BM[NH], holotype of melanurus; FMNH, 2; USNM, 3); Pichincha: Mindo, below, I (FMNH); Rio Caoní, 1 (FMNH).

\section{Philander opossum subspecies nov. 1}

Philander opossum subsp. nov. Pérez-Hernández, 1989:373, 377-VENEZUELA: Monagas (Cerro Papelón); Territorio Federal, Delta Amacuro (Tobesobe, Guayo; Los Gires; Güiniquina).

Distribution (Fig. 5)—The original description is that of an opossum with "a markedly different color pattern which could be suggestive of an undescribed subspecies for Venezuela." Pérez-Hernández (1989, p. 377) added that the "Orinoco Delta has a particular climate and vegetation, and can be defined biogeographically by an endemic subspecies of Philander opossum with a more melanistic coloration compared to other specimens of that species (Pérez-Hernández in preparation)."

Four specimens were examined by Pérez-Hernández, each from a different locality, one from Monagas, the others from Delta Amacuro. Five localities are shown on his map (Fig. 18, p. 404).

\section{Philander opossum subspecies nov.?}

Metachirus opossum (subsp. nov.?), J. A. Allen, 1916b:201-COLOMBIA: Meta (Villavicencio).

Metachirus opossum, Lyne, 1959:84-VENEZUELA: Mérida-vibrissae.

Philander opossum, Reig et al., 1977:197_part, VENEZUELA: Barinas (Guaquitas) - karyotype $(2 n=22)$. Tyndale-Biscoe, 1980:712-part, COLOMBIA: Meta (Villavicencio).

Philander opossum griscescens, Pérez-Hernández (not J. A. Allen), 1989:373-VENEZUELA: Barinas (Ticopore); Apure (Nulita); Aragua (Las Bonitas); Táchira (La Panchera); Trujillo (Motatán; Dividivi); Zulia (Encontrados; Alguacil; Churuli; Puerto Catatumbo).

Distribution-Map, Figure 5.

SPECIMENS EXAmined-10. VENEZUELA. Apure: La Blanquito, $4 \mathrm{~km} \mathrm{~N}$ Nula, 2 (USNM); Nulita, $3 \mathrm{~km}$ N Nula, 1 (USNM); Zulia: Encontrados, 2 (USNm). COLOMBIA. Boyacá: Rio Covaría, 1 (FMNH); Meta (Caney, Restrepo), 2 (AMNH); Finca El Capuchó, $38 \mathrm{~km}$ E Villavicencio, 2 (UKMNH).

\section{Philander opossum fuscogriseus}

\section{J. A. Allen}

Didelphys quica, Alston (not Temminck), 1880:198 MEXICO; GUATEMALA: (Dueñas; Cobán); COSTA RICA: (Tucurriqui).

Didelphys opossum, Thomas, 1888:331—part, GUATEMALA: (Vera Paz; Cobán).

Philander opossum, Tyndale-Biscoe, 1980-COLOMBIA: Valle del Cauca (Cali Buenaventura). Tuttle et al., 1981:233-PANAMÁ: Canal Zone (Barro Colorado Island); acoustical location of vocalizing frogs.

Metachirus fuscogriseus J. A. Allen, 1900:194; 1901b:213-NICARAGUA: (Greytown; Bluefields); variation.

Metachirus opossum fuscogriseus, J. A. Allen, 1911: 247-NICARAGUA: (Greytown). 


\begin{tabular}{lllll}
\hline $\begin{array}{c}\text { Condylobasal } \\
\text { Length }\end{array}$ & Zygomatic Arch & Preorbital Width & Postorbital Width & $\begin{array}{c}\text { Braincase } \\
\text { Width }\end{array}$ \\
\hline & & & & \\
68.9 & 36.6 & - & 8.7 & 22.3 \\
$69.1(64.6-71.0) 5$ & $36.1(33.8-37.8) 5$ & $13.1(12.3-13.9) 5$ & $9.3(8.8-9.7) 5$ & $21.4(20.4-23.5) 5$ \\
70.9 & 38.1 & - & 9.0 & 20.5 \\
71.9 & 37.3 & 13.7 & 9.3 & 22.0 \\
& & & & 20.9 \\
64.1 & 33.5 & - & 9.4 & 19.3 \\
\hline 65.8 & 34.2 & 12.5 & 8.4 & \\
\hline
\end{tabular}

TABLE 11. Extended (continued).

\begin{tabular}{|c|c|c|c|}
\hline Locality & Palatal Length & $\mathbf{i}-\mathbf{m}^{4}$ & $m^{1-4}$ \\
\hline \multicolumn{4}{|l|}{ Ecuador of $\delta$} \\
\hline Mindo, below & 40.8 & 36.2 & 13.4 \\
\hline Paramba & $41.8(38.6-43.0) 5$ & $36.3(34.7-37.4) 5$ & $13.6(13.3-14.0) 5$ \\
\hline San Javier & 42.9 & 37.2 & 13.8 \\
\hline Carondelet & 43.1 & 37.5 & 13.8 \\
\hline \multicolumn{4}{|l|}{ Ecuador $q ?$} \\
\hline Río Cauní & 38.8 & 34.6 & 13.0 \\
\hline San Javier & 39.9 & 34.8 & 13.0 \\
\hline
\end{tabular}

Metachirops fuscogriseus, Matschie, 1916:268-classification.

[Holothylax fuscogriseus] fuscogriseus, Cabrera, 1919:47-classification.

Metachirops opossum fuscogriseus, Enders, 1935: 410, Pl. 2 (animal)-PANAMÁ: (Barro Colorado); life history.

Philander opossum fuscogriseus, Goodwin, 1942: 113-NICARAGUA (type locality, Greytown); HONDURAS; characters. Hall and Kelson, 1959: 11 -classification; distribution in Middle America. Yates and Froehlich, 1984:512-HONDURAS (Río Plátano Biosphere Reserve, Gracias a Dios).

Metachirus fuscogriseus pallidus J. A. Allen, $1901 \mathrm{~b}$ : 213-MEXICO: Vera Cruz (type locality, Orizaba); holotype, male, skin and skull, U.S. National Museum of Natural History, no. 51858; collected 24 January 1894 (not 24 June 1894 as in original description) by E. W. Nelson and E. A. Goldman; Tabasco (Monte Cristo; Frontera; Teapa); Chiapas (Huehuetán; Chicharras); Vera Cruz (Orizaba; Montzorongo: Tuxtepec); Puebla (Metlaltoyuca).

[Holothylax fuscogriseus] pallidus, Cabrera, 1919: 47-classification.

Metachirops opossum pallidus, Goodwin, 1934:4 GUATEMALA; characters; distribution; habits.

Philander opossum pallidus, Baker, 1951:207MĖXICO: Tamaulipas (70 km S Ciudad Victoria, $2 \mathrm{~km} \mathrm{~W} \mathrm{El} \mathrm{Carrizo,} 2500 \mathrm{ft}$ ). Hall and Kelson, 1959:11-classification; distribution. Hall and Dalquest, 1963:195-MÈXICO: Vera Cruz (distribution, near sea level to $5600 \mathrm{ft}$ above); characters; habits; common names ("comadreja"; "raton tlac- uache"). Álvarez, 1963:394-MËXICO: Tamaulipas (E slope Sierra Madre Oriental to 2500 $\mathrm{ft})$; variation; habits.

Metachirus grisescens J. A. Allen, 1901b:217-COLOMBIA: Valle del Cauca ("Rio Cauca," type locality); holotype, female, skin and skull, American Museum of Natural History, no. 15072; collected June 1888 by J. H. Batty. Goodwin, 1953:227type history.

[Metachirops] grisescens, Matschie, 1916:268-classification.

[Holothlax grisescens] grisescens, Cabrera, 1919: 47-classification.

Metochirus opossum grisescens, J. A. Allen, 1916b: 201-COLOMBIA: Valle del Cauca (Río Frío).

Philander opossum grisescens, Goodwin, 1953:227classification; type history.

Metachirus opossum melantho Thomas, 1923:602COLOMBIA: Chocó (type locality, Condoto; altitude, $300 \mathrm{ft}$ ); holotype, young adult male, skin and skull, British Museum (Natural History), no. 1914.5.28.30; collected I February 1914 by H. G. F. Spurrell.

Metachirus opossum melanurus, J. A. Allen (not Thomas), 1912:73-COLOMBIA: Cauca (Cocal). J. A. Allen, 1916a:116; 1916b:201-COLOMBIA: Cauca (Cocal); Chocó (Bagadó); Antioquia (Alto Bonito). Cabrera, 1958:35-part, Metachirus opossum melantho Thomas in synonymy.

HolotyPE-Probably female, skin and skull, American Museum of Natural History, no. 9920/ 
TABLE 12. Measurements of Philander opossum fuscogriseus. Means (extremes in parentheses) and number of specimens.

\section{Locality Head and Body}

Ecuador $\hat{\partial} \hat{o}$

\section{Ventura}

Piñas

Bucay

Puente de Chimbo

Seboyal

Limón

Ecuador $q$ 우

Salvias, El Oro

Bucay

Ríos Chimbo-Coco

La Papaya

Colombia $\delta े$

Bagadó

Bellavista

Colombia $q$ q

Río Cauca

Alto Bonito

Bagadó

Panamá ơ ơ

Puerto Armuelles

La Zumbadora

Cocoli

Empire

Frijoles

Camp Piña

El Valle

Cerro Hoya

Isla Cebaco

El Volcán

Progreso

Cayo Agua

Isla Bastamentos

Boca del Drago

Almirante

Changuinola

Río Changena, Camp

Panamá $q$ ?

La Zumbadora

Camp Piña

Isla Cebaco

Progreso

Cayo Agua

Boca del Drago

Almirante

Changuinola

Costa Rica 000

Savanillas de Piri

Costa Rica $ㅇ$

Villa Quesada

Nicaragua ${ }^{*}{ }^{\circ}$

Río Escondido

Guatemala oै ${ }^{\circ}$
294, 308, 308

$-, 214$

292, 307, 307

247,278

260

$280,271,275$

237

$246,270,274$

259,271

256

$\overline{271}$

-

$-$

303

265,255

247,280

290

289

282 (255-305) 5

287

275

$205,288,290$

276

296

$286(261-300) 8$

285,300 , -

$285(261-301) 4$

274 (248-299) 8

279 (271-290) 9

275

$251,263,282$

$280(250-305) 9$

298

331

271 (255-280) 4

$282(255-294) 5$

$270(263-279) 3$

272

297

285

305

279
$261,268,293$

-, 261

261, 275, 257

250,240

262

$260,271,285$

246

$264,270,260$

249,240

268

$\overline{2} 94$

$-$

-

299

275,265

293, 295

300

275

$313(290-335) 5$

296

280

$278,280,302$

272

273

$283(270-290) 8$

300,300 , -

$291(269-300) 4$

$300(282-321) 8$

296 (275-332) 9

290

$278,302,253$

296 (263-320) 9

275

331

$264(250-270) 4$

$300(273-320) 5$

$300(286-312) 3$

280

291

275

320

310
$-$

38

$42,41,45$

43,43

38

$45,48,49$

$-, 41$

$44,48,47$

46,47

42

$42,43,45$

34

Ear

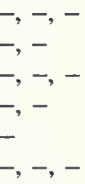

$-,-,-$

$-$,

26

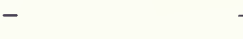

-

$49 \quad 39$

$44,45 \quad 35,33$

$43,45 \quad 37,35$

$41 \quad 32$

$46 \quad 35$

$49(46-52) 5 \quad 38 \quad(34-40) 5$

$43 \quad 35$

$42 \quad 36$

$45,46,50 \quad 36,35,37$

$48 \quad 33$

$44 \quad 36$

$45(38-48) 8 \quad 36 \quad(34-38) 8$

$45,47,-\quad 36,38,-$

$43(37-48) 4 \quad 38 \quad(36-40) 4$

$45(41-46) 8 \quad 36 \quad(33-40) 8$

$44(42-45) 9 \quad 36 \quad(34-37) 9$

40

35

$41,44,44 \quad 31,35,35$

$43(40-50) 9 \quad 35(34-38) 9$

$43 \quad 35$

$47 \quad 37$

$42(41-44) 4 \quad 34.5(34-35) 4$

$41(37-42) 5 \quad 37 \quad(35-40) 5$

$42(40-45) 3 \quad 36 \quad(32-39) 3$

$45 \quad 33$

$46 \quad 36$

$39 \quad 30$

46

44 


\section{Condylobasal}

$72.3,72.3 .75 .0$

$-61.9$

$71.2,71.4,73.5$

$64.7,68.5$

68.1

$68.4,71.9,73.0$

60.0

$63.0,67.0,69.3$

$62.9,66.4$

$-$

72.9

67.8

66.2

64.8

67.2

79.7

$70.9,76.4$

$72.0,77.2$

76.6

75.9

$77.8(74.4-81.5) 5$

73.5

69.6

$70.8,71.7,74.6$

69.7

73.5

$72.4(67.8-75.0) 8$

$73.0,73.3,75.2$

$74.0(72.0-75.3) 4$

$71.9(68.7-75.6) 8$

$73.8(68.0-76.3) 9$

73.2

$67.6,71.5,72.1$

$72.4(68.9-74.5) 9$

73.5

80.5

$67.6(64.3-70.2) 4$

$72.4(65.4-76.5) 5$

$68.6(65.7-72.2) 4$

67.8

78.7

66.1

76.5

69.7
39.7. 40.4, 41.1

$\overline{38} \overline{9}, 36.3,38.9$

32.2, 33.9

34.1

$33.6,40.4,49.1$

30.7

$33.0,32.4,33.0$

$32.5,33.8$

$-$

40.2

33.9

36.3

34. 1

32.9

41.2

$35.0,43.0$

$37.2,42.8$

39.8

42.3

40.5 (38.3-42.5) 5

38.5

36.6

$39.3,38.6,40.3$

35.9

37.5

38.3 (35.5-41.5) 8

$39.2,40.3,40.6$

$39.9(37.9-42.0) 4$

$37.5(34.6-41.2) 8$

38.0 (35.5-40.6) 9

38.9

$33.7,34.6,36.7$

$37.7(34.5-38.6) 9$

39.7

40.0

$35.4(33.9-35.2) 4$

$38.0(34.3-43.1) 5$

$36.1(34.4-39.1) 4$

35.5

43.0

32.7

35.9

$\begin{array}{ll}-,-,- & 9.3,9.2,9.5 \\ -,- & -,- \\ -,-,- & 8.7,9.1,9.2 \\ -,- & 8.5,9.3 \\ - & 8.7 \\ -,-,- & 8.8,9.2,-\end{array}$

$21.8,22.2,22.5$

$-,-$

$23.1,22.3,21.7$

$20.3,19.8$

21.1

$21.0,23.2,22.7$

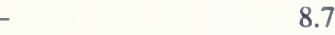

$-,-,-$

$-,-$

8.6, $9.3,8.9$

$9.3,8.4$

20.4

$20.4,21.1,21.3$

$22.0,20.5$

-

9.4

21.6

9.1

21.3

$\begin{array}{ll}9.4 & 22.1 \\ 8.8 & 20.6 \\ 8.4 & 19.6\end{array}$

15.4

$12.5,14.7$

$13.2,14.5$

13.7

13.3

$14.0(12.8-15.7) 6$

12.8

13.1

$12.8,13.7,13.3$

12.0

13.4

$13.2(11.8-14.5) 8$

$13.5,13.9,15.0$

$13.9(12.5-14.8) 4$

$13.1(12.2-15.2) 8$

$13.6(12.5-15.5) 9$

13.7

$12.0,12.7,13.1$

$12.9(11.6-13.8) 8$

13.8

14.0

$12.5(12.1-12.9) 4$

$12.7(11.8-13.3) 4$

$13.0(12.5-13.5) 4$

13.2

8.9

$8.4,8.7$

$8.8,8.6$

8.6

9.0

$9.0(8.6-9.4) 6$

8.9

8.9

$9.1,9.2,9.0$

8.4

9.1

$8.8(8.5-9.0) 8$

$9.0,9.0,8.7$

$8.7(8.5-8.9) 4$

$8.7(8.5-9.3) 8$

$8.9(8.6-9.4) 9$

8.9

$9.1,8.7,9.0$

$8.9(8.3-9.3) 8$

9.2

9.2

$8.7(8.5-8.8) 4$

$9.0(8.7-9.4) 5$

$8.9(8.8-9.1) 4$

8.9

20.1

$19.5,20.9$

$20.0,20.9$

20.0

23.5

$21.4(20.5-22.3) 6$

21.6

19.8

$20.1,20.1,21.0$

20.6

20.3

$20.0(19.1-21.4) 8$

$20.9,22.2,21.6$

$22.1(21.7-22.6) 4$

20.5 (19.2-22.7) 8

$20.3(19.8-20.8) 9$

22.2

$19.8,19.3,22.1$

$20.3(19.5-21.7) 8$

19.9

21.0

$19.1(18.7-19.4) 4$ $21.8(20.3-23.6) 5$ $20.2(19.5-21.3) 4$ 19.4

15.7

9.0

23.5

11.5

8.0

19.6 


\begin{tabular}{|c|c|c|c|c|c|}
\hline Locality & Head and Body & Tail & Hind Foot & & Ear \\
\hline \multicolumn{6}{|l|}{ Guatemala $\subsetneq$ } \\
\hline Los Amatos & - & - & - & - & \\
\hline \multicolumn{6}{|l|}{ México ơ ठे } \\
\hline $\begin{array}{l}\text { Palenque } \\
\text { Achotal }\end{array}$ & $\begin{array}{l}280 \\
269(252-292) 4\end{array}$ & $\begin{array}{l}316 \\
289(258-310) 4\end{array}$ & $\overline{44}(40-47) 4$ & $\overline{36}$ & $(34-37) 4$ \\
\hline \multicolumn{6}{|l|}{ México $q$ ? } \\
\hline $\begin{array}{l}\text { Tekom } \\
\text { Achotal }\end{array}$ & $\overline{300}$ & $\overline{300}$ & $\overline{45}$ & $-\overline{36}$ & \\
\hline
\end{tabular}

TABLE 12. Extended (continued).

\begin{tabular}{lllll}
\hline $\begin{array}{c}\text { Condylobasal } \\
\text { Length }\end{array}$ & Zygomatic Breadth & Preorbital Width & Postorbital Width & Braincase Width \\
\hline & & & & \\
70.0 & - & 11.8 & 9.0 & 21.8 \\
73.1 & - & 12.6 & & \\
$68.8(64.1-74.5) 5$ & $36.1(33.0-39.1) 5$ & $11.9(10.9-13.0) 5$ & 8.5 & 21.2 \\
& & & & \\
69.8 & 37.0 & 12.4 & 8.5 & 22.3 \\
71.8 & 39.0 & 12.4 & 8.9 & 21.9 \\
\hline
\end{tabular}

8252; presented by Albert Smith. The skin is labeled female, skull tag and box are labeled male. The skin is in poor condition but appears to be that of a female; the last molar is not fully erupted.

TYPE LOCALITY_-"Found [in New York, 11 January 1895] in a bunch of bananas in unloading a fruit steamer from a Central American port, most likely Colon ..." (J. A. Allen, 1900, p. 194); subsequently fixed as Greytown (= San Juan del Norte), Nicaragua, by Goodwin (1942, p. 113). According to Allen (1901b, p. 213), "a number of specimens from Greytown and Bluefields, Nicaragua agree with the type of this species, supposed to have come from a point somewhat further south on the coast." This statement does not constitute a fixation of type locality as claimed by Goodwin (1946, p. 284; 1953, p. 227), but Goodwin's designation is.

Distribution (Fig. 5)-In México the states of Tamaulipas on the east, Vera Cruz (Oaxaca, 1851/ 9705) on the west, south through Middle America into western Colombia, Andean valleys, and neighboring parts of Guayas in western Ecuador, to the Peruvian border. Altitudinal range sea level to $1000 \mathrm{~m}$ above. The distributional gap between the range of fuscogriseus in southwestern Colombia and that of $P$. opossum melanurus in northwestern Ecuador leaves moot the question of possible intergradation or sympatry between the taxa.

ChARACTERS-Intermediate in coloration between saturate melanurus and pale quica; conule $C$ of $\mathrm{m}^{3}$ comparatively well developed, as in $P$. andersoni.

COMPARISONS-Individuals of $P$. opossum fuscogriseus with dorsal surface like that of $P$. opossum melanurus and with tail entirely brown are usually much paler on ventral surface. Conule $C$ is most developed in fuscogriseus, least in the smaller, generally grayer quica.

Measurements-Tables 1 and 12.

TAXONOMY-The type of $M$. grisescens J. A. Allen from the Río Cauca valley in southwestern Colombia was distinguished from the Middle American opossums by its paler coloration and uniformly brown tail. Tail color of grisescens agrees with that of $P$. opossum melanurus Thomas, but the generally paler coloration identifies grisescens with western Colombian representatives of $P$. opossum fuscogriseus. Two specimens from Río Frío, Cauca Valley, which agree closely 


\section{Locality}

Ecuador $\delta$

Ventura

Piñas

Bucay

Puente de Chimbo

Seboyal

Limón

Ecuador \&?

Salvias, El Oro

Bucay

Ríos Chimbo-Coco

La Papaya

Colombia ơ $\delta$

Bagadó

Colombia \&?

Río Cauca

Alto Bonito

Bagadó

Panamá ơ ơ

Puerto Armuelles

La Zumbadora

Cocoli

Empire

Frijoles

Camp Piña

El Valle

Cerro Hoya

Isla Cebaco

El Volcán

Progreso

Cayo Agua

Isla Bastamentos

Boca del Drago

Almirante

Changuinola

Río Changena, Camp

Panamá $q$ ?

La Zumbadora

Camp Piña

Isla Cebaco

Progreso

Cayo Agua

Boca del Drago

Almirante

Changuinola

Costa Rica ơ oै

Savanillas de Piri

Costa Rica $q 9$

Villa Quesada

Nicaragua $\delta \vec{~} \delta$

Río Escondido

Guatemala of $\delta$
Palatal Length

i-m $\mathbf{m}^{4}$

$\mathrm{m}^{1-4}$

$43.7,43.2,44.8$

$-, 37.9$

$41.6,42.6,43.5$

$39.5,42.2$

39.7

$41.7,43.5,43.9$

37.0

$38.1,41.5,41.7$

$39.1,40.1$

40.5

44.3

41.2

40.6

38.8

41.7

48.7

$43.6,45.3$

$43.3,45.9$

46.7

46.4

$47.0(44.7-50.2) 6$

46.1

43.1

$43.5,44.1,45.4$

41.7

45.0

$43.8(41.9-47.2) 8$

$43.7,44.3,45.1$

$44.8(43.6-45.5) 4$

$43.5(42.5-44.6) 5$

$44.6(41.5-46.6) 9$

44.6

$41.0,44.5,44.4$

$44.6(42.7-46.0) 9$

44.8

48.7

$41.2(39.3-43.1) 4$

$43.6(39.6-45.8) 5$

$42.1(39.5-44.4) 3$

42.0

48.3

40.2

13.4

45.8

39.4

14.0

$37.0,38.7,38.5$

$38.3(36.0-39.5) 9$

38.5

41.2

$35.4(34.6-36.3) 4$

$36.8(34.5-38.7) 5$

$37.0(35.5-38.3) 4$

37.7

41.0

14.7

$14.8,15.2,15.3$

$14.5(13.5-15.1) 9$

14.5

15.3

$13.6(13.4-13.9) 4$

$13.4(13.1-14.0) 5$

$13.7(13.5-13.4) 4$

14.5

Concepción del Mar

42.0

38.1

14.2 
TABLE 12. Continued.

\begin{tabular}{|c|c|c|c|}
\hline Locality & Palatal Length & $\mathbf{i}-\mathbf{m}^{4}$ & $\mathrm{~m}^{1-4}$ \\
\hline \multicolumn{4}{|l|}{ Guatemala $q \uparrow$} \\
\hline Los Amatos & 42.3 & 36.0 & 13.4 \\
\hline \multicolumn{4}{|l|}{ México ơ oे } \\
\hline $\begin{array}{l}\text { Palenque } \\
\text { Achotal }\end{array}$ & $\begin{array}{l}43.3 \\
43.6(38.4-49.5) 5\end{array}$ & $\begin{array}{l}37.3 \\
37.2(35.7-38.8) 5\end{array}$ & $\begin{array}{l}14.1 \\
13.9(13.5-14.3) 5\end{array}$ \\
\hline \multicolumn{4}{|l|}{ México 9 ? } \\
\hline $\begin{array}{l}\text { Tekom } \\
\text { Achotal }\end{array}$ & $\begin{array}{l}42.2 \\
42.5\end{array}$ & $\begin{array}{l}37.6 \\
37.3\end{array}$ & $\begin{array}{l}13.4 \\
13.0\end{array}$ \\
\hline
\end{tabular}

with the type of grisescens, except for parti-colored tail, are undoubtedly fuscogriseus.

The type and only specimen of grisescens was collected June 1898 (not 1899) by J. H. Batty. Specific locality data were not given. Judged by his itinerary, reconstructed from other dated specimens (cf. Goodwin, 1953, p. 384), Batty must have taken the type in the upper Río Cauca Valley, probably between Cali and Río Frío.

Metachirus fuscogriseus pallidus J. A. Allen, from Orizaba, Vera Cruz, México, was distinguished from the Nicaraguan form of fuscogriseus by paler coloration, larger supraorbital patches, and paler and narrower crown. Measurements were given for 21 adults of both sexes from various localities in the Mexican states of Vera Cruz, Oaxaca, Chiapas, Puebla, and Tabasco.

Characters attributed to pallidus are demonstrable in other material from Vera Cruz but are not consistent in specimens from elsewhere throughout the range assigned to pallidus west of Honduras and Salvador. Variation in specimens from México, Guatemala, Belize, and Honduras and others reported from Salvador (Felten, 1958; Burt \& Stirton, 1961) falls well within the limits of variation of Costa Rican, Panamanian, and Colombian $P$. opossum fuscogriseus.

Metachirus opossum melantho Thomas from Condoto, northwestern Colombia, was compared with andersoni Osgood of the Peruvian Amazonas, whereas the genetically and geographically relevant grisescens, fuscogriseus, and melanurus were ignored in the original description. Typologically, melantho could be assigned to any one of the three forms named. Its nearly whitish underparts and sides and parti-colored tail, however, cast melantho with fuscogriseus. The Field Museum series from nearby Río Docampado, near the type locality of melantho, definitely belongs with fuscogriseus.
REMARKS - The more or less unpigmented terminal portion of the tail averages about $20 \%(0-$ $30 \%$ ) in Colombian and Ecuadorian opossums but becomes increasingly more extensive from Panamá into México. Álvarez (1963, p. 395) noted that the terminal whitish area averaged $53.1 \%$ (43.3$62.8 \%$ ) in 13 specimens from Tamaulipas and $38.7 \%(30.9-48.2 \%)$ in 14 specimens from Vera Cruz.

Bibliographic citations in the synonymies of Middle American representatives of $P$. opossum fuscogriseus are restricted to primary synonyms, selected taxonomies, and distributional references.

Specimens EXAmined-255. MĖXICO. Chiapas: Palenque, 1 (FMNH); Vera Cruz: Achotal, 9 (AMNH); Orizaba, holotype of pallidus J. A. Allen (USNM); Yucatán: Tekom, 1 (FMNH). HONDURAS. San Pedro, near Lake Ticamaya, 1 (FMNH). BELIZE. Central Farm, 3 (USNM). NICARAGUA. 4 (AMNH, holotype of fuscogriseus J. A. Allen, of unknown origin, but with type locality restricted to Greytown; USNM, 3); Escondido River, $50 \mathrm{mi}$ from Bluefields, 2 (USNM). COSTA RICA. Alajuela: San Carlos, 1 (FMNH); Villa Quesada, 1 (FMNH); San José: Fuentes, 1 (FMNH); Savanillas de Pirris, 1 (FMnh). GUATEMALA. Escuintla: Concepción del Mar, 2 (FMNH); Izabal: Bobos, 3 (FMNH); Los Amates, 1 (FMNH). PANAMÁ. Bocas del Toro: Almirante, 25 (USNM); Boca del Drago, 10 (USNM); Cayo Agua, 20 (USNM); Changuinola, 21 (USNM); Isla Bastamentos, 7 (USNM); Isla Colón, 2 (USNM); Río Changena Camp, 3 (USNM); $\mathrm{Ca}$ nal Zone: Cerro Campana, I (USNM); Cocolí, 4 (USNM); Empire, 1 (USNM); Fort Sherman, Camp Piña, 31 (USNM); Frijoles, Bahia Peninsula, 51/2 km WNW, 5 (USNM); Puma Island, 1 (USNM); no precise locality, 2 (USNM); Chiriquí: Boquerón, 1 (AMNH); Progreso, 4 (USNM); Volcán, $4100 \mathrm{ft}, 1$ (AMNH); Cocle: El Valle, 14 (USNM); Colón: Salud, 2 (USNM); Darién: Jaqué, 1 (USNM); Puerto Ar- 
muelles, 3 (USNM); Los Santos: Cerro Hoya, 5 (USNM); Panamá: Cerro Azul, 21 (USNM); Cerro Jefe, 4 (USNM); La Zumbadora, Gorgas Laboratory, 3 (USNM); Veraguas: Isla Cebaco, 14 (USNM); Isla Gobernado, 2 (USNM). COLOMBIA. Antioquia: Alto Bonito, 3 (AMNH); Bellavista, $4 \mathrm{~km} \mathrm{NE}$, I (FMNH); Caldas: Río Hondo, Samaná, I (FMNH); Canca: Cocal, upper Río San Juan, 1 (ANNH); La Boca, Río Saija, 3 (FMNH); Upper Río Cauca Valley, holotype of grisescens J. A. Allen (AMNH); Chocó: Bagadó, 8 (AMNH); Condoto, holotype of melantho Thomas (BM[NH]): Novita, I (AMNH); Río Docampado, 3 (FMNH); Río Sando, Río Baudó, 6 (FMNH); Valle del Cauca: Buenaventura, I (FMNH); Río Frío, 2 (AMNH). ECUADOR. El Oro: El Chiral, 2 (AMNh); Punta Santa Ana, I (AMNH); Salvias, I (AMNH); Guayas: Bucay, Río Chimbo, 8 (AMnh); Piñas, 4 (AMNh); Puente de Chimbo, 6 (AMNH); Ríos Chimbo-Coco, 6 (AMNH); Ventura, 3 (AMNh); Loja: Cebollal, 1 (AMNh); Manaví: La Papaya, I (AMNH); Río de Oro, I (AMNH); Río Pescado, 2 (AMNH); Limón, 1200 ft, 3 (AMNH, not located).

\section{Philander andersoni Osgood}

(Synonymies under subspecies headings)

Distribution (Fig. 5)-Amazonian basin of Perú, Ecuador, Colombia, Venezuela, and Acre in western Brasil; altitudinal range from near sea level to about $1900 \mathrm{~m}$ above.

Cranial Characters-Cranial characters as for the genus.

DENTAL Characters-Dental characters as for the genus.

COLORATION-Dorsal surface of trunk with broad, blackish (dark brown) longitudinal stripe, or entirely blackish, the dominant guard hairs usually coarse, elongate and uniformly colored or with a pale, narrow subterminal band; neck, chest, and belly often dark brown to blackish with gray basal portions of hairs showing through; lateral line, if present, restricted to a poorly defined hip patch; forefeet usually paler beneath than above, hind feet darker beneath than above; tail usually parti-colored with proximal half to four-fifths brown or blackish, remainder unpigmented, rarely entirely dark; ears usually blackish except sometimes with unpigmented band or patch extending from base to mid-center or margin.

Pelage-The muskrat-like pelage, particularly of the dorsum, more so in $P$. andersoni mcilhen- nyi than in $P$. andersoni andersomi, points to aquatic habits. The notably shorter, more woolly coat of $P$. opossum is less adapted for an aquatic environment.

The long-haired basal portion of the tail, according to Gardner and Patton (1972, p. 3), averages $26.6 \%$ of tail length in nine specimens of $P$. andersoni mcilhennyi from Balta and averages $16.9 \%$ in 10 specimens of $P$. opossum quica from the same locality. Ratios of a sampling from both species with $\mathrm{m}^{4}$ fully erupted, measured in the dry skin, are shown in Table 13. The data reveal that the thickly furred basal portion is most extensive in the Balta series of $P$. andersoni mcilhennyi and the Brasilian, French Guianan, and Surinam $P$. opossum opossum and, with few individual exceptions, is less in all others. The survey did not take into account seasonal differences, if any, in hair growth. Collectors' tail measurements recorded on field tags are usually reliable, or comparable, but may account for a few disparities in the ratios.

COMPARISONS-The broad, strongly defined dark middorsal longitudinal band or stripe of long coarse, apparently erectile hairs is the principal external character distinguishing $P$. andersoni from $P$. opossum. Sympatric and parapatric populations of the latter average slightly smaller in bodily and cranial dimensions (Table 14). Dorsum of $P$. opossum is nearly uniformly grayish brown with never more than a faint indication of dark dorsal band, which often may be an artifact of dry, folded-over skin, hind limbs grayish or buffy, not dark brown.

Sexual Dichromatism-Sexual dichromatism is most strongly marked in the Pucallpa, Río Ucayali, series of andersoni. In females, pheomelanin is saturate on underparts and dominant in the banding of the agouti hairs of other bodily parts. In males, the agouti pattern persists on underparts, with the pheomelanic subterminal bands thinly pigmented or pale buff. Irregular pheomelanic patches on belly and chest are paler than in females. Underparts of one of two female andersoni only from Santa Elena, Río Samiria, are completely saturate (i.e., monochromatic) pheomelanic.

Sexual dichromatism in remaining material is not as clear, but in general pheomelanin saturation in underparts and sides of body is dominant in females. In males, the trend is toward pigmentary dilution on all parts except for pheomelanin saturation of throat, midline of neck and chest, and pubic region. The intensity of sexual dichromatism varies from population to population. Adap- 
TABLE 13. Ratio of hairy basal portion of tail, in dry skin, to total length of tail (collector's measurement) in Philander andersoni and $P$. opossum. Figures are of individuals from each locality.

\begin{tabular}{lll}
\hline \multicolumn{1}{c}{ Taxon } & \multicolumn{1}{c}{ Locality } & Individual Ratios \\
\hline P. andersoni mcilhennvi & Balta, Perú & $26,28,33$ \\
P. andersoni andersoni & Pucallpa, Perú & $17,21,21,23,24$ \\
& Yurimaguas, Perú & 21 (holotype andersoni) \\
& Chanchamayo, Perú & 24 \\
P. opossum opossum & San Juan, Oxapampa, Perú & 27 \\
& Ecuador & $18,19,19,23,23$ \\
& Colombia & $17,19,20,20$ \\
& Venezuela & $17,19,22,24$ \\
& & $25,27,33$ \\
& Suriname & 28 \\
& Guyane Française & 20 \\
& Amapá, Brasil & 26 \\
& Pará, Brasil & 22 \\
& Goiás, Brasil & 25 \\
& Mato Grosso, Brasil & 24,24 \\
& Yarinacocha, Perú & 27 \\
& Moyobamba, Perú & 16,22 \\
& Pucallpa, Perú & $23,23,24$ \\
\hline
\end{tabular}

tive or behavioral correlation with sexual dichromatism is not readily interpretable. The affected parts of the body may or may not be used for display. The bright orange underparts of females and constrastingly brown marsupium are conspicuous to the human eye.

Geographic Variation-The melanistic Philander andersoni may have differentiated from the nearest ancestor of the already dark $P$. opossum, presumably during a Late or post-Pleistocene dry period in a forest refuge on the eastern base of the equatorial Andes. The postulated area incorporates the Napo refuge described by Haffer (1969). With return of humid, warmer climate, the species advanced north within its sylvan habitat into Colombia south across the Río Marañón into
Perú, east through gallery forests, into the forested highlands of southwestern Venezuela. In all outward spreading, the opossum became darker. One population isolated in the upper Río Purús south of the Marañón evolved into the extremely eumelanistic $P$. andersoni mcilhennyi.

Melanism in $P$. andersoni mcilhennyi is heaviest on head, trunk, tail base, and limbs except digits of forefeet. In the paler $P$. andersoni andersoni, the pigment is more concentrated on crown, nape, middorsal stripe, and over most of trunk. The geographic cline of increasing pigmentation is most notable in the Río Ucayali basin from Pucallpa south, east into headwaters of the Río Purús, and west into the Río Huallaga basin (Yurimaguas).

TABLE 14. Sympatric populations of Philander andersoni and P. opossum compared in size. Means (extremes in parentheses), specimens examined.

\begin{tabular}{|c|c|c|c|c|}
\hline & \multicolumn{2}{|c|}{ Balta, Perú } & \multicolumn{2}{|c|}{ Pucallpa, Perú } \\
\hline & $\begin{array}{c}\text { P. andersoni } \\
\text { mcilhennyi } \\
\left(1 \delta^{\star}, 3 \uparrow \wp\right)\end{array}$ & $\underset{(5 ठ \delta)}{\left.P . \delta^{\circ}\right)}$ & $\begin{array}{l}P \text { andersoni } \\
\text { andersoni } \\
(10,3 \circ \wp)\end{array}$ & 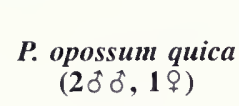 \\
\hline Head and body & $290 \quad(280-299) 4$ & $248 \quad(232-255) 5$ & $290.5 \quad(279-298) 4$ & $269 \quad(264-273) 3$ \\
\hline Condylobasal length & $73.9(73.5-74.4) 3$ & $65.0(62.5-67.5) 5$ & $71.2(70.2-73.1) 3$ & $64.7(64.2-65.1) 2$ \\
\hline Zygomatic breadth & $37.1(36.8-37.6) 3$ & $33.3(31.7-34.3) 5$ & $35.4(34.2-36.5) 3$ & $35.3(33.5-36.3) 3$ \\
\hline Braincase width & $21.1(21.0-21.2) 3$ & $19.1(18.4-19.6) 5$ & $20.6(20.2-20.8) 3$ & $20.7(19.9-21.4) 3$ \\
\hline Molar row & $15.2(14.9-15.3) 3$ & $13.5(12.9-14.3) 5$ & $14.2(13.7-14.6) 4$ & $12.9(12.5-13.1) 3$ \\
\hline
\end{tabular}


HABITS-According to notations on specimen labels, individuals of Philander andersoni andersoni had been shot out of trees and trapped on the ground.

At Tres Troncos, Río Caquetá, Colombia, two live suckling young, each about $6 \mathrm{~cm}$ in combined head and body length, were detached from their trapped, dead mother (FMNH 70985) and left to fend for themselves in camp, where I could observe them. At first, they tottered uncertainly on weak legs, but quickly adapted to life without mother. They ate ripe bananas, plantains, and papayas, lapped blood from animals being skinned, and ate some of the flesh. When not otherwise engaged the two fought with each other, the victor putting the loser to flight. Both animals disappeared after 2 days.

\section{Philander andersoni andersoni Osgood}

[?]D[idelphys] myosuros, Tschudi (not Temminck), 1844:14, 145, 151-PERÚ; female with well-developed pouch. Tschudi, 1844:250—PERÚ.

Metachirus andersoni Osgood, 1913:95; 1914:149PERÚ: Ucayali (type locality, "Yane Yacu," Río Paranapura, $8 \mathrm{mi}$ from Yurimaguas). Sanborn, 1947:215-type history.

$M$ [etachirops] andersoni, Matschie, 1916:268-classification.

[Holothylax] andersoni, Cabrera, 1919:47-classification.

M[etachirus] opossum andersoni, Thomas, 1923: 603 -classification.

Metachirus opossum andersoni, Thomas, 1928b: 294-PERU: Ucayali (Iquitos).

Philander opossum andersoni, Cabrera, 1958:34classification. Pérez-Hernández, 1989:373-VENEZUELA: Territorio Federal Amazonas (Caño Majagua; Cerro Cucurito; San Juan, Río Manapiari; Tamatama; Belén; Capibara; Campamento La Neblina; Mavaca; Sierra Parima).

P[hilander] opossum andersoni, Gardner and Patton, 1972:5-PERÚ: Ayacucho (Huanhuachayo; San José); characters; comparisons.

$P[$ hilander] $o[$ possum] . andersoni, Baker, 1974:135ECUADOR: Napo-Pastaza (Santa Cecilia).

Philander andersoni, Emmons and Feer, 1990:18part, “upper Amazon Basin of Venezuela, probably Colombia, Ecuador to Ucayali Department."

Metachirus opossum nigratus Thomas, 1923:603PERÚ: Junin (type locality, Utcuyacu, $1600 \mathrm{~m}$; Chanchamayo); type, male, skin and skull, British Museum (Natural History), no. 1900.7.7.62; collected 21 April 1900 by P. O. Simoms. Thomas, 1928a:250, footnote-PERÚ: Junín (Inañez). Gardner and Patton, 1972:5-"more likely a synonym of andersoni" than P. opossum canus, apud Cabrera, 1958.

Metachirus opossum Tate, 1939:161 -VENEZUELA: Amazonas (Mt. Duida).

Philander opossum, Gardner and Patton, part (not
Linnaeus), 1972:5-PERÚ: Ayacucho (San José, Río Santa Rosa, $1000 \mathrm{~m}$; Huanhuachayo, $1660 \mathrm{~m}$ ); comparisons. Reig et al., 1977:197-PERÚ: Ayacucho (San José, Río Santa Rosa: Huanhuachayo). Philander opossum canus Cabrera (part, not Osgood) 1958:35-nigratus Thomas only, a synonym.

TYPE-Male, skin and skull, Field Museum of Natural History, no. 19655; collected 11 September 1912 by M. P. Anderson.

TYPE LOCALITY - "Yane Yaku" [= Yanayacu], a chacra, or farm, on the Paranapura River about $8 \mathrm{mi}$ from Yurimaguas. The latter is on the Río Huallaga at the mouth of the Rio Paranapura (cf. Osgood, 1914. p. 147).

Distribution (Fig. 5)-Southeastern Colombia, eastern Ecuador, the Río Huallaga Valley in the Peruvian departments of Amazonas, Pasco, Junín, and Ayacucho, the Río Ucayali Valley, Ucayali, and the disjunct population of Territorio Federal Amazonas, Venezuela.

ChARACTERS-Dark middorsal band well defined from sides and continuing over rump to less than basal fifth of tail; hind limbs pale brown, grayish brown or buffy, forelimbs paler with digits usually unpigmented; ventral surface ochraceous orange to buffy or grayish buff.

COMPARISONS-Distinguished from Philander andersoni mcilhennyi mainly by paler coloration throughout and narrower, more sharply defined middorsal band; from sympatric representatives of $P$. opossum by larger size, dark middorsal band and darker coloration throughout.

Measurements-Tables 1 and 15.

VARIATION-The Andean andersoni from Huanhuachayo, Ayacucho, Perú, is dark like mcilhennyi but with dorsal pelage shorter, finer, dorsal band not clearly defined from sides, underparts and limbs paler, tail entirely pigmented except for some mottling on terminal portion. A second Andean specimen, from San José, Ayacucho, is slightly paler throughout except for a similarly dark brown tail. Its poorly defined dorsal band and nearly uniformly dark tail suggest intergradation with $P$. opossum. Nearest geographic representatives of $P$. opossum, however, from Cuzco and Madre de Dios are distinct. The third Andean specimen of andersoni from Chanchamayo, Junín, and another from San Juan, Oxapampa, also lack the well-defined blackish dorsal band of the subspecies. In all other respects, they agree. The condylobasal length of the last two, 76.3 and 81.2 $\mathrm{mm}$, respectively, are among the largest of the species. The heavily furred basal portion of the tail is notably extensive in the San Juan individ- 
TABLE 15. Measurements of Philander andersoni andersoni.

\begin{tabular}{|c|c|c|c|c|c|c|}
\hline Locality & Head and Body & Tail & Hind Foot & Ear & $\begin{array}{l}\text { Condylobasal } \\
\text { Length }\end{array}$ & $\begin{array}{l}\text { Zygomatic } \\
\text { Breadth }\end{array}$ \\
\hline \multicolumn{7}{|l|}{ Colombia $q q$} \\
\hline La Tagua & $270,274,264$ & $292,278,270$ & $37,40,37$ & $34,36,39$ & $65.9,69.1,64.6$ & $31.6,34.0,32.3$ \\
\hline \multicolumn{7}{|l|}{ Venezuela $\delta \delta^{\star}$} \\
\hline Capibara &,- 270 &,- 305 & 45,45 & 42,45 & $67.3,74.5$ & $32.3,37.6$ \\
\hline Tamatama & $266,-,-$ & $295,-,-$ & $44,42,-$ & $43,42,-$ &,,-- 73.7 & $36.3,-, 38.2$ \\
\hline Acanana & - & - & - & - & 71.4 & 36.4 \\
\hline Mount Duida & 243 & 285 & 45 & - & 67.9 & 36.5 \\
\hline Belén & - & - & - & - & 75.7 & 39.9 \\
\hline \multicolumn{7}{|l|}{ Venezuela $९$ ㅇ } \\
\hline Capibara & 265 & 285 & 41 & 40 & 67.3 & 32.3 \\
\hline Esmeralda & 258 & 267 & 45 & 41 & 67.6 & 33.7 \\
\hline Tamatama & 250 & 290 & 36 & 33 & - & - \\
\hline Belén & 225 & 300 & 41 & 38 & 67.7 & 36.5 \\
\hline Merey & 250 & 255 & 41 & - & 69.1 & 35.0 \\
\hline \multicolumn{7}{|l|}{ Perú ơ $\sigma^{\star}$} \\
\hline Chanchamayo & 280 & 275 & 47 & 30 & 76.3 & 38.1 \\
\hline San José & 275 & 300 & 48 & 34 & 71.3 & 36.6 \\
\hline Yurimaguas & 284 & 288 & 40 & - & 76.3 & 37.8 \\
\hline Pucallpa & 279 & 313 & 40 & 35 & 73.1 & 36.5 \\
\hline Santa Luisa & 267,304 & 265,282 & 40,42 & 33,35 &,- 73.6 &,- 39.3 \\
\hline Río Curaray & - & - & - & - & $73.0,68.9$ & $37.0,-$ \\
\hline \multicolumn{7}{|l|}{ Perú $q q$} \\
\hline Huanhuachavo & 270 & 300 & 45 & 35 & 71.7 & 36.5 \\
\hline Utcuyacu & 265 & 275 & 40 & 35 & 68.2 & 32.3 \\
\hline Oxapampa & 300 & 332 & 51 & 36 & 81.2 & 40.0 \\
\hline Yurimaguas & 275 & 278 & 35 & - & 68.6 & 30.8 \\
\hline Pucallpa & $298,295,290$ & $279,280,290$ & $36,40,40$ & $37,35,38$ & $-, 70.2,70.3$ & $-, 35.5,34.2$ \\
\hline Santa Elena & 278,277 & 270,263 & 41,40 & 35,33 & $68.6,67.1$ & $33.3,31.7$ \\
\hline Río Curaray &,,--- &,,--- &,,--- &,,--- & $68.7,65.3,72.3$ & $36.7,32.9,35.8$ \\
\hline \multicolumn{7}{|l|}{ Ecuador ơ $\delta^{\circ}$} \\
\hline Pindo Yacu & 250,300 & 255,315 & 40,40 & 34,38 & $68.3,76.5$ & $34.0,39.8$ \\
\hline Yana Rumi & 278 & 285 & 40 & 32 & 72.4 & 38.5 \\
\hline Montalvo & 250,264 & 280,262 & 35,37 & 35,35 & $74.9,66.7$ & $37.5,34.9$ \\
\hline \multicolumn{7}{|l|}{ Ecuador $q q$} \\
\hline San José & - & - & - & - & 68.5 & 34.8 \\
\hline
\end{tabular}

ual. A skull from Chanchamayo in the British Museum (Natural History), assigned to nigratus (= andersoni) by Thomas (1923, p. 603), measures $80.7 \mathrm{~mm}$ in condylobasal length.

Two specimens from Santa Elena, Río Samiria, between the Ucayali and Huallaga, are intermediate between typical andersoni, the Pucallpa series of andersoni, and the Balta topotypes of incilhennyi. The same departure from heavier pigmentation continues westward into the Peruvian Andes (Junín). In Amazonian Venezuela, however, individuals of $P$. andersoni andersoni are blackish but with underparts and limbs pale (Capibara, USNM 406960; Belém, USNM 388412).

TAXONOMIC History-The dark Peruvian phi- lander identified by Tschudi (1844, p. 145) as $D i$ delphys myosuros Temminck may well be the earliest mention of the form described by Osgood as $P$. andersoni. Tschudi (1844, pp. 14, 144, 151) also noted the occurrence in Perú of the thencalled $D$. opossum Linnaeus but mentioned no specimens.

In his review of four-eyed pouched opossums, Thomas (1923, p. 603) treated all recognized forms as subspecies of Philander (then Metachirus) opossum. His description of Metachirus opossum nigratus (1923, p. 603) conforms to that of a sexually dichromatic male $P$. andersoni andersoni.

SympatRY - H. Hinse, who collected in the Pucallpa region, took both $P$. andersoni andersoni 
TABLE 15. Exiended.

\begin{tabular}{|c|c|c|c|c|c|}
\hline $\begin{array}{l}\text { Preorbital } \\
\text { Width }\end{array}$ & $\begin{array}{l}\text { Postorbital } \\
\text { Width }\end{array}$ & $\begin{array}{l}\text { Braincase } \\
\text { Width }\end{array}$ & $\begin{array}{l}\text { Palatal } \\
\text { Length }\end{array}$ & $\mathrm{i}-\mathrm{m}^{4}$ & $m^{1-4}$ \\
\hline $10.4,12.5,10.6$ & $8.3 .8 .8,8.9$ & $19.9,21.1,19.7$ & $40.1,41.1,39.7$ & $35.5,35.4,34.2$ & $13.6,13.0,12.6$ \\
\hline $\begin{array}{l}12.3,14.3 \\
12.6,-, 13.7 \\
12.6 \\
-14.4\end{array}$ & $\begin{array}{l}8.8,8.5 \\
8.6,8.4,9.4 \\
8.2 \\
8.9 \\
8.6\end{array}$ & $\begin{array}{l}19.6,22.0 \\
19.6,-, 22.4 \\
21.0 \\
20.8 \\
22.4\end{array}$ & $\begin{array}{l}41.2,44.6 \\
42.6,43.8,42.6 \\
42.5 \\
40.8 \\
44.3\end{array}$ & $\begin{array}{l}36.5,38.1 \\
37.8,38.2,38.5 \\
37.0 \\
36.8 \\
38.2\end{array}$ & $\begin{array}{l}13.5,13.8 \\
14.0,14.0,14.1 \\
13.4 \\
13.5 \\
13.5\end{array}$ \\
\hline $\begin{array}{l}12.3 \\
12.3 \\
12.8 \\
12.6 \\
-\end{array}$ & $\begin{array}{l}8.8 \\
8.7 \\
8.8 \\
8.0 \\
8.5\end{array}$ & $\begin{array}{l}19.6 \\
20.0 \\
- \\
19.9 \\
19.8\end{array}$ & $\begin{array}{l}41.2 \\
41.2 \\
41.3 \\
40.5 \\
41.2\end{array}$ & $\begin{array}{l}36.5 \\
36.7 \\
36.3 \\
36.3 \\
36.2\end{array}$ & $\begin{array}{l}13.5 \\
12.9 \\
13.6 \\
13.2 \\
13.4\end{array}$ \\
\hline $\begin{array}{l}- \\
- \\
13.7 \\
13.5 \\
-,- \\
-,-\end{array}$ & $\begin{array}{l}9.2 \\
9.2 \\
9.3 \\
8.4 \\
-.9 .0 \\
8.0 .-\end{array}$ & $\begin{array}{l}- \\
21.6 \\
21.5 \\
20.8 \\
-, 22.5 \\
22.6,-\end{array}$ & $\begin{array}{l}48.9 \\
43.4 \\
45.8 \\
44.5 \\
40.9,44.2 \\
44.0,42.4\end{array}$ & $\begin{array}{l}43.3 \\
39.1 \\
39.3 \\
38.4 \\
36.0,38.9 \\
38.9,37.2\end{array}$ & $\begin{array}{l}16.0 \\
14.5 \\
13.7 \\
14.6 \\
13.2,14.4 \\
14.4,14.0\end{array}$ \\
\hline $\begin{array}{l}14.0 \\
12.5 \\
15.3 \\
13.0 \\
-, 13.7,12.7 \\
13.0,12.9 \\
-,-,-\end{array}$ & $\begin{array}{l}9.8 \\
9.4 \\
10.1 \\
8.0 \\
-, 8.8,8.7 \\
9.3,8.2 \\
9.1,8.5,8.9\end{array}$ & $\begin{array}{l}21.0 \\
19.6 \\
23.9 \\
19.8 \\
-, 20.2,20.7 \\
20.5,19.3 \\
22.3,20.5,22.1\end{array}$ & $\begin{array}{l}44.9 \\
42.5 \\
49.6 \\
41.8 \\
-.42 .0,43.0 \\
42.2,41.0 \\
41.6,39.7,44.6\end{array}$ & $\begin{array}{l}38.8 \\
38.7 \\
42.9 \\
36.8 \\
-, 36.4,37.2 \\
37.3,37.0 \\
36.5,35.2,36.8\end{array}$ & $\begin{array}{l}14.3 \\
14.6 \\
15.0 \\
14.0 \\
14.2,14.1,13.7 \\
14.8,14.2 \\
14.0,13.4,13.5\end{array}$ \\
\hline $\begin{array}{l}11.8,14.5 \\
13.6 \\
13.9,13.2\end{array}$ & $\begin{array}{l}8.7,8.7 \\
8.6 \\
8.8,9.0\end{array}$ & $\begin{array}{l}21.2,21.0 \\
21.2 \\
19.9,20.0\end{array}$ & $\begin{array}{l}41.8,46.5 \\
43.3 \\
45.0,40.3\end{array}$ & $\begin{array}{l}35.2,38.6 \\
38.0 \\
37.8,35.8\end{array}$ & $\begin{array}{l}12.0,13.4 \\
14.3 \\
13.9,13.6\end{array}$ \\
\hline - & 9.0 & 21.3 & 42.0 & 37.5 & 14.0 \\
\hline
\end{tabular}

and the smaller $P$. opossum quica $59 \mathrm{~km} \mathrm{~W}$ of Pucallpa, October 1971 and 1972 and November 1971, but only $P$. opossum $59 \mathrm{~km} \mathrm{NE}$ of Pucallpa (September 1972) and $59 \mathrm{~km} \mathrm{SW}$ of Pucallpa (October 1972).

Specimens Examined-82. ECUADOR. Napo: Río Napo, 3 (BM[NH]); Río Yana Rumi, I (FMNH); San José, below, 2 (AMNH); Pastaza: Copataza, 3 (AMNH, 2; FMNH); Río Pindo Yacu, 2 (FMNH); Montalvo, Río Bobonaza, 2 (FMNH); Sarayacu, 1 (Amnh), COLOMBIA. Caquetá: Tres Troncos, Rio Caquetá, 4 (FMNH); Putumayo: 17 km N Puerto Asis, I (UKMNH). VENEZUELA. Territorio Federal Amazonas: Capibara, Casiquiare Canal, 7 (USNM); Belén, Río Cunucunumá, 1 (USNM); Acan- aña, Río Cunucunumá, I (USMN); Tamatama, 9 (USNM); Playa del Río Base, Mt. Duida, 2 (AMNh); Merey, Río Casiquiare, 1 (AmNH); San Juan, Río Manapiare, 2 (USNM); Esmeralda, 2 (USNM). PERÚ. Amazonas: $5 \mathrm{mi}$ W Huampani, Río Cenepa, 1 (Mvzuc); La Poza, Río Santiago, 1 (mvzuc); Ayacucho: Huanhuachayo, 1 (LSUMz); San José, Río Santa Rosa, I (Lsumz); Junín: Chanchamayo, 2 (BM[NH]: fmnh); Inañez, 1 $(\mathrm{BM}[\mathrm{NH}])$; Utcuyacu, 1, holotype of nigratus Thomas (Bм[NH]); Ucayali: lquitos, $3(\mathrm{BM}[\mathrm{NH}], 2$; AMNH); Santa Elena, Río Samiria, 2 (FMNH); Santa Luisa, 2 (FMNH); Yana Yacu, Yurimaguas, 3, including holotype of andersoni Osgood (FMNH); Orosa, 1 (FMNH); Río Curaray, mouth, 7 (AMNH); 
TABLE 16. Measurements of Philander andersoni mcilhennyi.

\begin{tabular}{|c|c|c|c|c|c|c|}
\hline Locality & Head and Body & Tail & Hind Foot & Ear & $\begin{array}{l}\text { Condylobasal } \\
\text { Length }\end{array}$ & $\begin{array}{c}\text { Zygomatic } \\
\text { Breadth }\end{array}$ \\
\hline \multicolumn{7}{|l|}{ Perú ơ ơ } \\
\hline Balta & 299 & 276 & 43 & 41 & 74.4 & 37.6 \\
\hline \multicolumn{7}{|l|}{ Perú q } \\
\hline Balta & 290,292 & 304,305 & 44,43 & 42,35 & $73.9,73 .+$ & $36.8,36.8$ \\
\hline
\end{tabular}

Lago Mirañes, Río Napo, 2 (BM[NH]); Pucallpa, Río Ucayali, 8 (UsNm); Pasco: San Juan, Oxapampa, 1 (USNM).

\section{Philander andersoni mcilhennyi \\ Gardner and Patton}

Philander mcilhennyi Gardner and Patton, 1972:2, Fig. I (furred tail base), Fig. 2 (skull). Reig et al., 1977: 197, 212, Pl. 2E (karyotype)-PERÚ: Ucayali (Balta, Río Curanja); karyotype $(2 n=22)$. Patton et al., 1995 [1996]:7, 20, 21-BRASIL: Amazonas (Alto Rio Urucu); relationships based on molecular evidence.

Philander andersoni Engstrom and Gardner, 1988:231P. mcilhenni [sic] a synonym. Emmons and Feer, 1990:18 - part, PERÚ: Ucayali; BRASIL: Acre.

TYPE-Adult female, skin and skull, Louisiana State University, no. 16395 (originally no. 11541); collected 10 April 1971 by Alfred L. Gardner.

Type Locality - Balta, Río Curanja, upper Río Purús, Ucayali, Perú; altitude about $300 \mathrm{~m}$.

Distribution (Fig. 5)-Known only from the banks of the upper Río Purús, Ucayali, Perú. Emmons and Feer (1990, p. 18) included Acre, Brasil, in the geographic range based on specimen in the U.S. National Museum of Natural History.

Characters-Darkest of four-eyed opossums; dorsal band broad and more or less differentiated from sides, its long, coarse dorsal pelage often manelike, extending over basal fourth or fifth of tail; fore- and hind limbs brown except for contrastingly pale or unpigmented digits of forefeet; ventral surface of body dominantly dark brown, variously mixed with gray; throat, chest, and belly often marked with large, irregular, whitish, buffy, or ochraceous patches; two-fifths to three-fifths of terminal portion of tail sharply contrasted pale brown or whitish.

COMPARISONS-Distinguished from $P$. andersoni andersoni by darker coloration throughout, pelage of dorsal band longer, wider, coarser; tail base bushy, 4-6 cm; from sympatric representa- tives of $P$. opossum by larger size, overall darker coloration, and broad, dark dorsal band.

Measurements-Tables 1 and 16.

REMARKS-Morphologically, $P$. andersoni mcilhennyi intergrades completely with $P$. andersoni andersoni through the Pucallpa, Río Ucayali (8 specimens), and Santa Elena, Río Samiria (2 specimens). The two groups are assigned to $P$. andersoni andersoni.

The geographic origin of the tissue studied by Patton et al. (1995 [1996]) and identified as that of $P$. mcilhennyi lies well within the area of projected sympatry between $P$. andersoni mcilhenny $i$ and $P$. opossum quica (map, Fig. 5). Patton et al. did not explain why they prefer the junior synonym mcilhennyi as the species name for the dark gray four-eyed opossum, $P$. andersoni.

Hutterer et al. (1995, p. 15) believe that Philander mcilhennyi and $P$. andersoni are distinct species, but comparisons were made only between $P$. mcilhennyi and $P$. opossum, which are indeed distinct. They point to differences in the color pattern of the head and the clipped genal vibrissae of $P$. opossum. Normally, all facial vibrissae in $P$. opossum are as in $P$. mcilhennyi $(=P$. andersoni mcilhennyi). They also claim that andersoni and opossum are "probably separately confined to the western and eastern cordillera [sic] of the Andes." In fact, both subspecies are confined to the eastern side of the Andes in Perú (infra map, Fig. 5).

SPECIMENS ExAmined-4. PERÚ. Ucayali: Balta, 4, including holotype of mcilhennyi Gardner and Patton (LSUMZ).

\section{Behavior of the Four-Eyed Pouched Opossums (Philander opossum)}

The activities and traits are arranged alphabetically. 


\begin{tabular}{cccccc}
\hline $\begin{array}{c}\text { Preorbital } \\
\text { Width }\end{array}$ & $\begin{array}{c}\text { Postorbital } \\
\text { Width }\end{array}$ & $\begin{array}{c}\text { Braincase } \\
\text { Width }\end{array}$ & Palatal Length & $\mathbf{i}^{-\mathbf{m}^{\mathbf{4}}}$ & \\
\hline \\
14.0 & 9.0 & 21.2 & 45.1 & 39.8 & 15.3 \\
$14.7,13.9$ & $9.2,9.2$ & $21.0,21.0$ & $44.5,44.7$ & $39.7,39.3$ & $15.3,14.9$ \\
\hline
\end{tabular}

Aggression, reaction, defense

Associations

Barbara: The pale-phase four-eyed opossum

Blood values

Fat storage

Foraging

Grooming

Habitat

Home range and territoriality

Hydrotropism

Locomotion

Longevity

Maternal care

Mobility

Nest

Population density
Breeding

Cannibalism

Diet

Display

Enemies, predators

Eye shine

Population dynamics

Posture

Reproduction

Sleep

Sociability

Spontaneous bleeding

Stress

Tail transport

Taming

Thermoregulation

Vocalization

Weight

\section{Aggression, Reaction, Defense}

Enders (1935, pp. 410, 412; Canal Zone, PANAMÁ)

"Next to the Brown Opossum [Metachirus nudicaudatus], this is the fiercest fighter of the opossums studied. Allen's opossum is ready to fight at all times and no amount of handling appears to diminish its resentment."

One shipped live in the same box with a $\mathrm{Me}$ tachirus of approximately the same size "had been thoroughly cowed by the more aggressive Metachirus and showed signs of having been bitten severely about the head and neck, while the aggressor was scatheless."

\section{Goldman (1920, p. 52; PANAMÁ)}

"Unlike Didelphis when taken in steel traps these opossums are always ready to fight savagely."

Miller (in J. A. Allen, 1916c, p. 589; Mato Grosso, BRASIL)

"When trapped it does not feign death but fights viciously, biting at any object within reach and holding on until its strength fails. They do not hesitate to bite iron bars or steel traps even though it may break the teeth."

Wilson (1970; Barro Colorado Island, PANAMÁ)

"About 8:07 PM on the evening of 3 February, 1969, I observed an aggressive encounter between Didelphis marsupialis and Philander opossum. The incident occurred in the laboratory clearing near the animal house on Barro Colorado Island, Panama Canal Zone. A loud hissing noise attracted my attention to a small Didelphis and a Philander that were circling slowly and snapping at each other on the lawn. Neither showed signs of wounds and both moved adeptly. Each attempted to grasp the rostrum of its opponent. Whenever the Didelphis secured this grasp, it quickly threw the Philander to the ground and held it down with both forefeet and hindfeet, at the same time biting it in the head. Twice in 2 minutes, the Philander broke loose and tried to escape but each time was attacked by the Didelphis. Soon the Philander was effectively pinned on its back and by $8: 22$ PM had died from the repeated bites on the head, or at least no longer responded to the attacker."

\section{Hershkovitz (field notes; COLOMBIA)}

The usual reaction to apparent or presumed aggression is a bipodal or tripodal stance, mouthopen hissing, often with a lurch forward of the body as if to attack.

\section{Associations}

Hershkovitz (field notes; COLOMBIA)

Philander opossum was captured in the same 
trap lines with species of Metachirus, Chironectes, Didelphis, Caluromys, Monodelphis, and mouse opossums (Marmosinae). All inhabit the same forests.

\section{Barbara: The Pale-Phase Four-Eyed Opossum}

\section{Hershkovitz (field notes, October 1992)}

A pale-phase female was live-trapped 17 October 1992 in a wooded area about $1200 \mathrm{~m}$ above sea level in the Parque Nacional do Caparaó, eastern Minas Gerais, Brasil. Her nearly completely closed pouch sheltered six attached young, possibly no more than 1 week old. She was kept in her cage for observation of behavior and litter development. Dubbed Barbara, she accepted captive life with equanimity, making no attempt to escape even when the door of her cage was opened for feeding or cleaning. She ate everything served, whether beef, chicken legs, insects, peanut butter, bananas, or mice. Live mice dropped into the cage were instantly attacked, the head and neck seized and crunched in her jaws. The mice were completely devoured with nothing left of flesh, entrails, skin, or bones.

Barbara slept most of the day curled up under the cut grass provided for nesting. Prepared food or raw fruit given during the day was usually consumed at night. Water was imbibed at all hours. Although normally nocturnal, should a live insect or mouse be dropped into the cage during the day, Barbara would awake with a start and make an instant kill and a fast meal. She easily devoured an entire mouse 10 to $15 \%$ of her own weight and looked for more.

When wanting food Barbara would fix her eyes on the nearest person, raise her muzzle, and sniff expectantly.

On one occasion Barbara left intact hide and fur of a mouse otherwise completely consumed.

Barbara washed her face frequently after and during meals. One or both paws were used. Grooming forequarters, sides, and underparts usually followed in that order without particular attention to the pouch and none to the young.

On the 26th of October, head-rump length of the six still-attached pouch young ranged from 1 to $2 \mathrm{~cm}$. Mother and offspring were transported the next day to Rio de Janeiro, where facilities were available for their care.

\section{Blood Values}

Tyndale-Biscoe (1980, p. 718; COLOMBIA)

Blood values measured at sea level (Buenaventura), $500 \mathrm{~m}$ above (Villavicencio), and $1600 \mathrm{~m}$ above (Cali) revealed correlations with age and altitude. Hemoglobin concentrations and hematocrit values were lowest at lowest altitudes, and those for pouch young lower than for adults.

\section{Breeding}

Natterer (in Pelzeln, 1883, p. 110-Sepetiba, Rio de Janeiro, BRASIL)

Female captured 8 March 1818 with five naked, blind pouch young.

Female captured 29 September 1822 in Ipanema, Rio de Janeiro, with four blind pouch young ("D. dichrura").

Miller (in J. A. Allen, 1916c, pp. 563, 589-Urucum, Mato Grosso, BRASIL)

"Number of young is usually small, between three and eight."

Davis (1947, p. 2-Teresópolis, Rio de Janeiro, BRASIL)

Seven of 32 females captured in Teresópolis had pouch young, average number, 4.5. Two females each had seven young and seven nipples in pouch. Breeding begins in August and continues until February.

Fonseca and Kierulff (1989, p. 119-Atlantic forest, Minas Gerais, BRASIL)

Female captured February and another in August had five pouch young each.

Cerqueira et al. (1993-Rio de Janeiro, Restinga de Barra de Maricá, BRASIL)

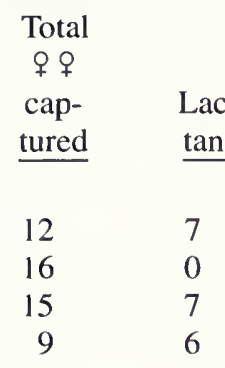


Tyndale-Biscoe (1980, p. 713-Buenaventura, Valle, $10 \mathrm{~m}$; near Villavicencio, Meta, $500 \mathrm{~m}$; near Cali, Valle, $1000 \mathrm{~m}$, COLOMBIA)

Four females captured August and September 1971 , one pregnant, three lactating, and each with five pouch young.

Charles-Dominique et al. (1981, p. 425-Ile de Cayenne, GUYANE FRANÇAISE)

Litter size, 4.24 (1-7).

Litters mature in 70 days.

Lactating females three of 16 in June, two of 13 in July, zero of nine in August.

Pouch young survival and food availability: first litter following dry season, 97\% $(n=36)$; second litters, $97 \%(n=38)$; third litters, food scarce, $28 \%(n=67)$.

Interval between litters, 90 days.

Sexual maturity, 150 days.

Seventy-nine percent of October-November population ( $n=42)$ born in preceding season.

Ovarian cycle inhibited by lactation but not by gestation.

Charles-Dominique (1983, p. 409, GUYANE FRANÇAISE)

Didelphoid breeding is continuous throughout the year, but successful reproduction is dependent on a rich diet for the female and is seasonal. Up to three successive litters may be produced in 1 year. The third litter "generally occurs at the beginning of the period of food scarcity and in most cases the females lose weight and young die in the pouch at a more or less early stage. Exceptionally, another litter can follow and abort, but generally reproduction is interrupted until the beginning of good recrudescence of fruiting. Among the Didelphidae, $P$. opossum behaves more like an $r$-strategist in favoring rapid population increase in an unstable environment."

\section{Charles-Dominique (1983, p. 409, GUYANE FRANÇAISE)}

Comparison of breeding conditions between Philander opossum and Caluromys philander of Guyane Française, two similar-sized species occupying different ecological niches:

\section{Philander opossum}

1. Mainly terrestrial

\section{Caluromys philander}

1. Almost entirely arboreal
2. Omnivorous but largely frugivorous

3. Food availability variable in undergrowth where foraging is done

4. Birth to weaning 68-75 days

5. Size weanling, 50$75 \mathrm{~g}$

6. Age at sexual maturity, 6 months

7. Up to three successive litters in same year (third litter mostly pouchaborted)

8. Behavior as $r$-strategist $^{1}$

9. Nesting period, 8 15 days

10. Litter size not more than 7
2. Mostly frugivorous, nectarivorous

3. Food availability less variable in upper strata where foraging is done

4. Birth to weaning 110-125 days

5. Size weanling, 50$75 \mathrm{~g}$

6. Age at sexual maturity, almost 10 months

7. Two successive litters in same year

8. Behavior more like that of $k$-strategist ${ }^{2}$

9. Nesting period, 30-45 days

10. Litter size not more than 7

\footnotetext{
$1 r$ strategist: $r$-selected species, selection favoring a rapid rate of population increase. Typical of species that colonize short-lived environments or of species that undergo large fluctuation in population size.

${ }^{2} k$ strategist: $k$-selected species, selection producing superior competitive ability in stable, predictable environments in which rapid population growth is unimportant as the population is maintained at or near the carrying capacity of the habitat.
}

\section{Atramentowicz (1986a, p. 125, GUYANE FRAN- ÇAISE)}

During a study period from September 1978 through October 1979 in Cayenne, two successive litters were produced between September and June. Some females, however, produced three litters in the same period. The difference is attributed to an abundance of food and a shortened nesting period. In the event of a food shortage, milk production ceases. Death of lactating young starved at the nipple is termed pouch abortion.

Average adult weight, $400 \mathrm{~g}$.

\section{Atramentowicz (1986a, p. 123, GUYANE FRAN- ÇAISE)}

During a 26-month (1978-1982) investigation of didelphoid breeding, "animals were live trapped, marked and then released. Caluromys philander, Philander opossum, and Didelphis marsupialis are nocturnal, with a mixed frugivo- 
TABLE 17. "Reproductive condition of 22 adult females of Philander opossum from Nicaragua" (Phillips \& Jones, 1969, p. 345).

\begin{tabular}{|c|c|c|c|}
\hline Date of Capture & Weight (g) & Pouch Young & Measurements ${ }^{1}(\mathrm{~mm})$ \\
\hline 16 February & - & None & - \\
\hline 2 March & - & 7 & $20.0^{2}$ \\
\hline $4 \mathrm{March}$ & - & 7 & $\mathrm{~nm}^{3}$ \\
\hline 5 March & - & 7 & $\mathrm{~nm}$ \\
\hline $8 \mathrm{March}$ & - & 6 & $\mathrm{~nm}$ \\
\hline 8 March & - & 6 & $\mathrm{~nm}$ \\
\hline $10 \mathrm{March}$ & - & 7 & $24.0^{2}$ \\
\hline $13 \mathrm{March}$ & - & 3 & $43.0^{2}$ \\
\hline 17 March & 355.0 & $5\left(20^{\circ}, 3 q\right)$ & 44.8 \\
\hline 29 March & - & 4 young, out of pouch $(3 \hat{\sigma}, 1 \uparrow)$ & 107.0 \\
\hline 31 March & 420.2 & $5(10,4 q)$ & 35.2 \\
\hline 31 March & 402.4 & $5\left(3 \sigma^{\wedge}, 2 q\right)$ & 31.0 \\
\hline 2 April & 546.3 & $7\left(50^{\circ}, 29\right)$ & 63.8 \\
\hline 2 April & 674.5 & $6\left(40^{\circ}, 29\right)$ & 65.5 \\
\hline 2 April & 361.8 & $6\left(20^{\circ}, 49\right)$ & 38.5 \\
\hline 15 April & - & $7(40,3 q)$ & 48.0 \\
\hline 15 April & 521.3 & $7\left(30^{\pi}, 4 \%\right)$ & 44.0 \\
\hline 15 April & 464.9 & $7\left(3 \sigma^{\circ}, 4 \uparrow\right)$ & 47.1 \\
\hline 24 April & 352.1 & $6\left(4 \sigma^{\star}, 2 \uparrow\right)$ & 57.0 \\
\hline 2 July & 256.3 & $7\left(20^{*}, 5 \uparrow\right)$ & 40.5 \\
\hline 6 July & 550.0 & Lactating, no pouch young & - \\
\hline 15 July & 590.0 & $7(5 \delta, 19,1 ?)$ & 89.0 \\
\hline 27 July & 282.7 & 5 & $\mathrm{~nm}$ \\
\hline
\end{tabular}

\footnotetext{
${ }^{1}$ Head and body combined.

2 "Probably crown-rump length."

${ }^{3} \mathrm{~nm}=$ no measurement.
}

rous and insectivorous diet. The first species is arboreal, the others mainly terrestrial, but may be seen climbing to the canopy. The percentage of lactating females in the studied population has been compared to food supply in terms of the availability of fruiting food plants."

"The collected data show a direct relation between these two factors. Breeding occurs throughout the year but food scarcity may interrupt reproduction either with no births or "pouch-abortions' (death of pouch-young) by starvation for lack of milk production."

Atramentowicz (1986b, p. 140, GUYANE FRANÇAISE)

Youngest lactating female, age 8 months.

Pouch young 4.2 (2-7); 179 litters.

Two births between September 1978 and October 1979.

\section{Atramentowicz (1988, p. 55, GUYANE FRAN- ÇAISE)}

"Fruits are essential during lactation, the sugars they contain helping the females to cover their increased energy need during this period. On weaning, the inhibition of the ovarian cycle is removed and a new litter may be produced during the next 15 days. In fact, the ovarian cycle is not inhibited during gestation as in placental mammals, but by the suckling which begins after birth when the embryos attach themselves to the teats."

\section{Phillips and Jones (1969, NICARAGUA)}

Size, weight, length, and dates of pouch young of 22 litters are shown in Table 17. Dates range from 16 February to 27 July; pouch litter size, 6.05 (3-7) 21 ; sex ratios in 13 of the litters with 78 young was $41(53 \%)$ oै oे, $37(47 \%)$ q $q$; average head and body length per litter increased from 20.0 to $107.0 \mathrm{~mm}$ within above time frame.

\section{Biggers (1966, p. 264, NICARAGUA)}

Seventy-three live-trapped males had spermatozoa in testis and epididymis throughout the year; testis weights are lower from September to December inclusive than in remainder of year.

Pouch young found January to October inclusive with peaks in January, May, and August.

Mature animals obtained January to November inclusive. No males trapped in December. No dif- 
ference in weight of testes of mature animals throughout the year.

Discrepancies noted in paragraphs one and two above are confusing. Perhaps the mixture of data for Philander and Didelphis is the cause. Biggers refers readers to the original compilation of data.

\section{Enders (1935, p. 411, PANAMÁ)}

Only nonbreeding females captured January to April; starting July. "females with litters outnumber those without; litters of two, four, and five were observed."

\section{Enders (1966, p. 198, PANAMÁ)}

"Observations on 18 captured Philander indicate that their breeding season ... begins with onset of the dry season in February."

"The largest litter encountered was five."

Fleming (1973, p. 444, Canal Zone, PANAMÁ)

Females were reproductively active during June, July, and September through December in 1966 and during February through April and June in 1967. Females carrying pouch young or lactating in October and November were anoestrus. Two, possibly more, litters are produced per season.

"Males of $P$. opossum are sexually mature at a body length of 230 millimeters or greater ... adult males probably are sexually active throughout the year."

Litter size 4.6 (2-7) 34; sex ratio $22(45 \%)$ o oे, $27(55 \%)$ 우.

\section{Seba (1734, p. 57, SURINAME)}

\section{Eight pouch young were counted.}

Seba and Miller (above) are the only authors to record eight pouch young. Maximum number in all other cases is seven, in agreement with number of nipples. Philander, unlike Didelphis and others, may not produce more young than can be suckled.

\section{Cannibalism}

Charles-Dominique (1983, p. 405; Cayenne, GUYANE FRANÇAISE)

Many cases of cannibalism have been observed in captivity of weaned young being eaten by other young and the mother.

\section{Density}

Charles-Dominique et al. (1981, p. 383, Cayenne, GUYANE FRANÇAISE)

100-200 individuals per $\mathrm{km}^{2}$.

\section{Diet}

Temminck (1827, p. 38)

It preys on small birds and insects but also eats fruit. In captivity it feeds on meat.

\section{Pelzeln (1883, p. 111, BRASIL)}

Insects found in stomach.

\section{Goldman (1920, p. 52; PANAMÁ)}

"The stomach of one taken at Gatun was well filled with fragments of crabs. Stomach contents of several others at the same locality were filled with fragments of birds alone, or of birds including their feathers, and crabs intermixed."

\section{Fleming (1972, p. 623; Canal Zone, PANAMÁ)}

"Stomachs of the four-eyed opossum contained unidentified plant material, the pulp of Corozo oleifera nuts, insect and fresh water shrimp remains, murid rodent fur, and the tail of a young spiny rat (Proechimys semispinosus). One individual was seen feeding on live bats caught in a mist net."

\section{Schomburgk (1840, p. 344; GUYANA)}

"They are very destructive to poultry and likewise to fruit. They are often found on those savannahs where the wild pine (Bromellia sp. ?) flourishes, to the fruit of which they appear to be partial."

\section{Enders (1935, p. 410; Canal Zone, PANAMÁ)}

They "are accused of being poultry- and birdkillers. ... In captivity they were more carnivorous than any of the other opossums observed. They ate meat of all kinds including ant-eater, carcasses of rodents, grasshoppers, and eggs in preference to any kind of fruit, although they did eat banana, papaya, pineapple, and figs. One hungry individual, while fearful of the hand offering a ripe banana, nevertheless sniffed from a safe distance while saliva dripped from its jaws." 
Dalquest (1953, p. 19; eastern San Luis Potosí, MÉXICO)

"One animal became entangled when it attempted to climb a net to obtain an entrapped bat, others were taken in traps baited with decayed meat, and two were shot while they were feeding on small wild figs. Remains of insects were usually present in the stomachs of the specimens taken."

\section{Anthony in Goodwin (1934, p. 5; GUATEMALA)}

"I was told that these little opossums are often killed in the sugar house [trapiche] and that they are quite fond of sweets. ... At Finca Capres, several times I surprised them at night, feeding on the ripe [bananas].... At Chipore ... one was attracted by bait composed chiefly of peanut butter and rolled oats."

\section{Hall and Dalquest (1963, p. 198; Veracruz, MÉX- ICO)}

"It has been seen feeding on sweet-lemons, jobo plums, and the fruit of the Chico Zapote ( $\mathrm{Sa}$ pote achras) source of chewing gum." One opossum "was seen at the base of a large, hollow fig tree. The upper part of the hollow in the tree served as a retreat for a colony of the large fruit bat, Artibeus jamaicensis. Bats were bringing small green figs into the hollow and feeding on them. Parts of the fruit, varying in size from almost whole figs to mere shreds, were dropped by the bats. The four-eyed opossum was feeding on these bits of figs."

The opossum "followed our trap lines, eating mice and other small mammals that had been captured. These opossums were easily trapped by using flesh, preferably much decayed, for bait."

Dubost (in Charles-Dominique, 1971, p. 197, GUYANE FRANÇAISE)

Philander eats tree exudate, and like the primate Cebuella (Callitrichidae) or Microcebus (Lemuridae) uses its teeth to reopen healed openings in tree trunks to renew the flow of sap.

\section{Charles-Dominique (1983, pp. 397, 398; Cay- enne, GUYANE FRANÇAISE)}

The diet is opportunistic, consisting of fruits, flowers, nectaries, insects, earthworms, small vertebrates, and carrion. Sympatric D. marsupialis subsists on about the same; Marmosa murina pre- fers fruits but takes earthworms and small vertebrates. Marmosa cinerea [ = Micoureus demerarae $]$ and $C$. philander are vegetarian.

"The period of food scarcity determined both by records of fruit and insect abundance and also by body weight changes in the marsupials begins in May, is most critical during June-July-August, and ends in September. Data collected in 1976. and from 1976 to 1982 by the Institut Pasteur de Cayenne (variation in weight and reproductive condition of marsupials) suggest that this period of food scarcity is a regular phenomenon, probably correlated with seasonal variations in rainfall."

Charles-Dominique et al. (1981, p. 376; Cayenne, GUYANE FRANÇAISE)

"Eighty percent of stomach contents consisted of a great variety of prey. During the rainy season. earthworms, some of which are as much as $80 \mathrm{~cm}$ long, forced above ground, made up a large part of the diet.

"Fallen fruit consumed were mainly Attalea regia, Virola melinonii, Virola sebifera, Virola surinamensis, Ocotea puberula, Richardella macrophylla, Ficus spp., Cordia exaltata, Simarouba amara, Protium heptaphyllum. In general the pulp is eaten with only the smallest seeds (Ficus, etc.) swallowed, the larger ones spit out. The ground level flowering of the Balanophoracea from January to June is actively sought for the nectar. Loses weight during dry season (September-November in the years 1978, 1979) when fruit is scarce, fatten when fruit is abundant."

\section{Tyndale-Biscoe (1980, p. 716; COLOMBIA)}

"They would eat fruit and vegetables provided as well as meat; chicken bones were held in the hands and the ends chewed."

Atramentowicz (1986a, p. 125; Cayenne, GUYANE FRANÇAISE)

Eats ripe pulpy fruit, insects, and small prey such as earthworms, frogs, etc.

Atramentowicz (1988, p. 48; Cayenne, GUYANE FRANÇAISE)

Stomach contents of Philander opossum consist of $50 \%$ fruit with the remainder invertebrates, small vertebrates, and carrion. A preference is shown for ripe fruit fallen to the ground. The fruit consumed represented 44 species in 21 families. 
It was characterized by a fleshy pulp with a high percentage of water. The color may be bright or dull, size large or small, shape variable. The pulp may be rich in sugar or lipids but poor in nitrogen, which the animal finds in animal prey.

\section{Display}

Hershkovitz (field notes)

The supraorbital spots are conspicuous at night, at least to the sharp-eyed (see "Sleep," p. 77). Conspicuous supraorbital marking displayed by both nocturnal and diurnal animals may serve for recognition between conspecifics. I find no difference in markings between the sexes, and the signs are usually absent or faint in juvenals.

\section{Enemies, Predators}

Most likely predators are the eyra cat (Herpailurus jagouaroundi) and smaller spotted cats, large mustelids such as the tayra (Eira barbara) and grison (Galictis vittata), foxes, large owls, and snakes. Husson (1978, p. 26) found remains of a Philander opossum in the stomach of a garden tree boa (Corallis enhydris) in Suriname. Schomburgk (1840, p. 344) reported that "they are sometimes eaten by the Creoles and Indians [of Guyana], but as they have a rank and disagreeable smell I doubt if they would prove palatable to us."

Wilson (1970, p. 386) reported an encounter in Panamá between Didelphis marsupialis and Philander opossum with the former overcoming and devouring skin, flesh, bones, and brain of the latter (see details under "Aggression, Reaction, Defense").

\section{Eye Shine}

Anthony in Goodwin (1934, p. 5; GUATEMALA)

"In the beams of the carbide hunting lamps, their eyes were extremely bright, gleaming white, like electric lamps, easily seen at one hundred yards or more."

Enders (1935, p. 412; Canal Zone, PANAMÁ)

The eyes of [Philander] show up as a reddish yellow under the hunting lamps while the eyes of [Metachirus] show red."

\section{Hershkovitz (field notes)}

The eyes of Philander, like those of other didelphoids and Metachirus, usually glow bright orange when reflecting the beam of a battery-powered flashlight. There is individual variation in tone of eye shine depending on the amount of environmental light, intensity of directed light, and angle of reflection. These factors and the uncontrolled conditions make it difficult to find significant differences in color of didelphid eye shine. More important for identification is distance between the eyes and animal gait as seen by the reflected light.

\section{Fat Storage}

Hall and Dalquest (1963, p. 198; Veracruz, MÉX$\mathrm{ICO}$ )

"In the lowlands these opossums seldom were fat, but in the highlands, at 5000 feet elevation and higher, in winter, they had a deep layer of yellow [!] fat immediately beneath the skin."

\section{Foraging (Table 18)}

Charles-Dominique et al. (1981; Cayenne, GUYANE FRANÇAISE)

Of a total of 294 individuals live-trapped or tracked by radio, 185 were located from 0 to $6 \mathrm{~m}$ above ground in the following situations: on ground 70\%; fallen $\operatorname{logs} 7 \%$; small tree trunks $2 \%$; branches $6 \%$; lianas or vines $15 \%$.

Charles-Dominique et al. (1981, p. 343; Cayenne, GUYANE FRANÇAISE)

Nearly always forages on the ground; of 100 individuals live-trapped only three were captured above ground.

Charles-Dominique (1983, p. 397; Cayenne, GUYANE FRANÇAISE)

See Table 18 for body mass and above-ground foraging by five syntopic species of opossums. 
TABLE 18. Body mass and above-ground foraging by five syntopic species of opossums.

\begin{tabular}{lcl}
\hline \multicolumn{1}{c}{ Taxon } & Weight $(\mathbf{g})$ & \multicolumn{1}{c}{ Substrate } \\
\hline Marmosa murina & 40 & Low (shrub) \\
Marmosa cinerea & & \\
[= Micoureus demerarae $]$ & 80 & High \\
Caluromys philander & 300 & High \\
Philander opossum & 400 & Low (essentially ground level) \\
Didelphis marsupialis & 1,000 & Low (may climb to feed on fruit) \\
\hline
\end{tabular}

\section{Grooming}

Hershkovitz (field notes, January 30, 1941; northern COLOMBIA)

A captive washed face with hands mouselike; groomed remainder of body with tongue catlike.

Charles-Dominique (1983, p. 407; Cayenne, GUYANE FRANÇAISE)

"We have never observed any allogrooming between [caged] adults, even during mating periods."

\section{Habitat}

Hall and Dalquest (1963, p. 196; Veracruz, MÉXICO)

". . . ranges throughout the tropics of Veracruz. At the extreme upper edge of the upper humid division of the Tropical Life-zone, it lives along cold, clear streams at the edge of the oak belt. Lower down but still in the upper humid division, it was found along rivers and streams that flowed through dense jungle, where the tall, broad-leafed trees were thickly hung with orchids, vines, mosses and bromeliads. The four-eyed opossum was found living in the thickets bordering the broad rivers of the coastal plain, in the arid division of the Tropical Life-zone, and along the marshy shores of rivers and streams of the lower humid division of the Tropical Life-zone, in the southern part of the state.

"Most of our specimens were taken on the very shores of rivers or streams . . however ... the species is not confined to such habitat... seven kilometers west of Potero, workers discovered a family of four young animals in a field of sugar cane, several kilometers from the nearest water at that time of the year. At Jimba, 350 feet elevation, in southern Veracruz, a four-eyed opossum was taken from a tree on a hillside fully three kilo- meters from the nearest water. These records are unusual, however. Against them are nearly 30 records from in and near water."

\section{Dalquest (1953, p. 19; eastern San Luis Potosí, MÉXICO)}

"Found only in the tropics of the eastern part of the state ... they are taken in dense vegetation growing along the shores of streams and rivers at elevations between 400 and 12,000 feet [more likely 1200 feet]. At El Salto the water is cold and flows swiftly between rocky canyon walls; opossums were found in thickets of ferns, vines, and low, woody plants. Near Huichihuayan, where the water is warmer and sluggish, opossums were taken in thickets of thorny bamboo."

\section{Goodwin (1946, p. 284; COSTA RICA)}

"Frequenting forested country from sea level up to about 4000 feet, he is most common at low elevations."

\section{Davis (1947, p. 2; Teresópolis, BRASIL)}

"Found more commonly in moist situations although individuals may wander through nearly any kind of vegetation."

\section{Enders (1935, p. 410; Barro Colorado Island,} PANAMÁ)

"They are not common on the Island for while they enter traps without hesitation none were taken; but many were captured at Alhajuela in habitats similar to those on the Island ... why they are not abundant on the Island is a puzzle."

\section{Schomburgk (1848, p. 777; GUYANA)}

"It lives mainly in the coastal forests bordering the plantation. During the day it sleeps most of the time in its haunt under tree roots or in hollow trees." 
Natterer (in Pelzeln, 1883, p. 111; southeastern BRASIL)

“. . Fashions its nest from leaves on low trees."

Miller (in J. A. Allen, 1916c, p. 589; Mato Grosso, BRASIL)

". . . is found in deep forests. I have never known it to venture near houses for the purpose of robbing hen-roosts and nests like its larger relative [Didelphis marsupialis], although it may do so."

\section{Handley (1976, p. 8; VENEZUELA)}

Lowlands of western and southern Venezuela. 46 specimens: on the ground, $98 \%$; on a $\log , 2 \%$; near streams and other moist areas, $100 \%$; evergreen forest, 91\%; orchards, croplands, yards, open areas, 9\%; elevation $24-324 \mathrm{~m}$.

Hall and Dalquest (1963, p. 198; Vera Cruz, MÉXICO)

“. . . are usually seen or trapped on the ground, but are sometimes seen in trees." They prefer the vicinity of streams and seem to be as much at home in rivers as on their banks.

Enders (1935, pp. 410, 411; Barro Colorado Island, Canal Zone, PANAMÁ)

None "were taken in the Island but one was observed high up on a limb on Zetek Trail. ... While a good climber [it] was usually encountered upon the ground, on or under logs. It is probably more terrestrial in habit than any of the other opossums studied excepting possibly Didelphis. This might be surmised from its build, which is well adapted to terrestrial locomotion."

\section{Davis (1947, p. 2; Teresópolis, BRASIL)}

"These opossums seldom climb but are partial to fallen logs and windfalls."

Farris (1950, p. 259; PANAMÁ)

"About eighteen females were collected in grass fields about 47 miles from Panama City in a location that is low and damp all the year."

Charles-Dominique (1983, p. 419; Cayenne, GUYANE FRANÇAISE)

The frequency of encounter with Philander is
10 to 16 times greater in secondary than in primary forest.

\section{Husson (1978, p. 26; SURINAME)}

Some of these opossums were found under the floor of a home at Lelydorp.

Atramentowicz (1986a, p. 123; Cayenne, GUYANE FRANÇAISE)

Philander opossum is mainly terrestrial "but may be seen climbing to the canopy" in the second growth forest.

Da Fonseca and Kierulff (1989, p. 118; Atlantic forest, Minas Gerais, BRASIL)

"Fourteen individuals were caught during the [17-month] course of this study. The occurrence of this large didelphid is apparently tied to the presence of standing or running water. . . As only a few transects occurred close to streams, this may explain the low trapping success for this species."

"The species also proved to be primarily terrestrial with only $17 \%$ of captures in arboreal traps, and $7 \%$ of individuals climbed trees after being released."

\section{Unpublished (VENEZUELA)}

Capture sites recorded by members of the Smithsonian Venezuelan Project (SVP) on field tags of captured specimens are summarized below:

\begin{tabular}{lc} 
& $N$ \\
\cline { 2 - 2 } $\begin{array}{l}\text { Live-trapped in banana } \\
\text { grove }\end{array}$ & 1 \\
$\begin{array}{l}\text { Snap-trapped on forest } \\
\text { floor }\end{array}$ & 2 \\
Live-trapped on ground & 9 \\
Snap-trapped in river & 1 \\
Snap-trapped in tree & 1 \\
Shot in tree in forest & 1 \\
Shot in hollow tree near \\
river \\
Shot in tree alongside river \\
Total
\end{tabular}

All Philander andersoni were taken in the Territorio Federal Amazonas. The P. opossum were captured in the state of Bolivar, where different ecological conditions prevail (Handley, 1976). It seems that $P$. andersoni is at least as arboreal as it is terrestrial, with perhaps a preference for trees 
overhanging rivers. This type of habitat restricts enemy attacks to the forested side of the river and provides the opossum with the opportunity of escaping by diving into the water. The muskrat-like dorsal pelage of $P$. andersoni mcilhennyi and many individuals of $P$. andersoni andersoni seems to be better adapted than that of $P$. opossum for aquatic life. Where the two species occur together, as in Balta and along the Río Ucayali in Perú, $P$. andersoni may be more arboreal-aquatic than terrestrial, whereas $P$. opossum may be more terrestrial-aquatic than arboreal.

\section{Home Range and Territoriality}

\section{Miles et al. (1981a, BRASIL)}

A small, transparent plastic spool, $6 \mathrm{~cm}$ long with $2.2 \mathrm{~mm}$ internal and $2.4 \mathrm{~mm}$ external diameters, was wound with double-strand teryline thread. The spool was attached to a captive Philander opossum, and the free end of the thread was attached to the capture trap or nearby vegetation. As the released animal traveled through the night the thread unwound. The thread tracked during the day led to the diurnal nest or refuge. Distance traveled was calculated from the weight of the spool before release of the animal and after recapture.

Of 21 released animals, 16 were retrieved. The calculated distance for 15 retrievals averaged 438 $\mathrm{m}(93-1000 \mathrm{~m})$. This compared with a mean of $801 \mathrm{~m}$ (51-2450 m) for Didelphis marsupialis.

\section{Charles-Dominique (1983, p. 401; Cayenne, GUYANE FRANÇAISE)}

Philander explores about $25,000 \mathrm{~m}^{2}$ during the night.

"By systematically trapping, marking and releasing we have observed regular loss of individuals, compensated by immigrants from other areas (juveniles and adults of both sexes). Trapping was conducted using Tomahawk and Sherman traps placed on the ground and special 'home-made' traps set at 15 to $20 \mathrm{~m}$ in trees; traps were baited with bananas ... The following data concern new-arrivals [in an over 20-ha study area] from May to October 1979, after 8 months of regular trapping and marking": May, eight immigrants; June, 2; July, 1; August, 3; September, 4; October, 3.

"Migration seems to be more important in $P$. opossum than in C. philander - after one year, the entire population of $P$. opossum had been replaced by new individuals, but only one-third of the original C. philander population was replaced."

Atramentowicz (1986b, p. 145; GUYANE FRANÇAISE)

Didelphoids are solitary, not territorial, and many individuals are sedentary. Duration of residence in study area is compared with Caluromys philander, Philander opossum, and Didelphis marsupialis (Table 19).

\section{Charles-Dominique (1983, p. 418)}

All American opossums are nocturnal and solitary except during mating and maternal care of young. Territory is not defended. Home ranges overlap between many individuals, male and female, but with close contact avoided. There are no social bonds between adults apart from short copulatory periods; there is no allogrooming.

\section{Hydrotropism (see also Habitat)}

\section{Hershkovitz (1962, field notes; SURINAME)}

A female with 3 pouch young taken live 20 January 1962 from a snap trap in Lelydorpplan was caged for observation. The animal never recovered from the effects of the blow on the head received from the trap. She slept most of the time and when awake walked slowly with poor coordination. She permitted herself to be petted and seemed to enjoy it, but the docility probably owed to her weakened condition. She ate the insects fed to her and seemed to require large amounts of water. A Marmosa murina held captive at the same time derived all its water from fruit.

The Philander escaped twice from her cage. On each occasion she sought out the nearest dark, cool, moist refuge. The first time she curled up in an empty but still-wet canvas bucket in the kitchen. The second time, after I had moved to a frame house in La Poule, she snuggled into a dark, wet corner behind a primitive flush toilet.

Fonseca and Kierulff (1989, p. 118; Atlantic forest, Minas Gerais, BRASIL)

"The occurrence is apparently tied to the presence of standing or running water."

Tyndale-Biscoe (1980, p. 716; COLOMBIA)

"All these animals were caught in second 
growth forest close to streams, in several caves less than a meter from water. It was thus interesting to observe that a pair in captivity would invariably defecate into the water container rather than elsewhere in the enclosure."

\section{Locomotion}

Dalquest (1953, p. 19; eastern San Luis Potosí, MÉXICO)

They "are swift in their actions. For the most part they hunt on the ground, but they climb readily, often to considerable heights, and are skillful swimmers. Animals twice escaped capture by diving into the water of the Rio Naranjos and swimming away beneath the surface."

\section{Crespo (1950, p. 6: Misiones, ARGENTINA)}

They "are excellent swimmers, water apparently being their preferred habitat. I have watched them at night swimming swiftly even upstream against the current in rivers such as the Uruguaí."

\section{Hall and Dalquest (1963, pp. 197, 198; MÉXICO)}

"Philander is quick and active. Trapped individuals are able to jump about and twirl in surprising fashion..." The animal "is an agile climber and a skillful swimmer."

"Along the Río Atoyac several four-eyed opossums were taken in a trap set beneath the water level, at the base of a cut-bank. They could have reached the trap only by swimming."

"... A four-eyed opossum was seen just before midnight, running swiftly over the larger, rounded boulders (six to 18 inches in diameter) along the river bank. When frightened [by us] the animal turned [from the river's bank] and made a smooth, clean dive into the swift water, and as it did not reappear, must have swum away underwater."

\section{Hershkovitz (field notes)}

The usual didelphid locomotor pattern on the ground is a measured walking gait, and in climbing an alternating movement of the grasping hands and feet with support of the clinging, prehensile tail. The principal running gait on or above ground is a trot. I have never seen a didelphid leap or bound except in rapid escape. In descending a tree trunk the animal will proceed head first or simply drop to the ground.
Hershkovitz (1941, field notes; northern COLOMBIA)

The prehensile tail was used for swinging the body from limb to limb, the hands for grasping the next higher support. The animal would also use the tip of its tail for clasping an overhead limb, then climb its tail until it could grasp the limb above with its hands. Carrying an opossum by its tail can be hazardous. Marmosids habitually use the tail for climbing.

When attacking live prey, the opossum springs for throat or head with lightning speed.

\section{Longevity}

Farris (1950, p. 258)

Life span in captivity, 3 years, 6 months.

Atramentowicz (1986b, p. 140; GUYANE FRANÇAISE)

Based on tooth wear, longevity of wild Philander opossum and Didelphis marsupialis was calculated as 2.5 years: of Caluromys philander, as 3.5 years. Captive Caluromys philander (Brunoy, France) lived to 6 years. Maximum life expectancy of Didelphis marsupialis was said to be less than 36 months.

\section{Maternal Care}

Seba $(1734$, p. 57)

"The mother does not remove the young from her pouch before they are old enough to enjoy the light of day. When the time is right she retreats to a lookout to assure the safety of her young. She then opens the pouch and permits the offspring to emerge into the sunlight and play with her. At the slightest suspicion of danger she calls her young with a warning cry that sounds tik, rik, tik. The young respond immediately by returning to the pouch whereupon the mother runs to a hiding place."

COMMENT-Seba actually beheld the mother and her brood at one time or another. The fanciful description of their relationship (translation), however, must have been confected long after the supposed facts.

Didelphids are nocturnal, but in captivity, especially as household pets, as Seba's opossums 
must have been, they generally adjust to the hours they are fed.

\section{Nest}

\section{Goldman (1920, p. 52; PANAMÁ)}

"A nest of one of these opossums was found three feet from the ground on a fallen log. The $\log$ lay in the dense thicket of an old clearing and was heavily overhung with vines and bushes. The nest, globular in form and about a foot in diameter, was placed in a well hidden spot among the vines. It was made entirely of the banana-like leaves of a native plant rather neatly laid together. The opening at one end faced outward along the log. ... The nest cavity was clean and about the size of the animal's body."

\section{Carriker (in J. A. Allen, 1911, p. 247; Yuruán, VENEZUELA)}

"The female with a litter of young was taken out of the hollow stump in a grass field in the [forest] clearing."

\section{Hall and Dalquest (1963, p. 197; Veracruz, MÉX- ICO)}

"Several four-eyed opossums were taken in a trail leading from a dense thicket . . . to a stream ten feet away.... A hole, about five inches in diameter that led downward beneath the roots of a tree was discovered near the center of the thicket. This seemed to be the home of at least one of the opossums."

Two "were found in a nest that they had constructed in the palm thatch of the roofs of abandoned houses. These nests consisted of a handful of dry leaves, pushed in between the layers of palm fronds. From outside, a distinct spherical or oval lump in the thatch marked the site of the nest. Inside the house we could see no trace of the nests."

A nest "was found in a cavity in the side of a piece of tree trunk, 15 inches in diameter and three feet long, that was suspended in the air by vines. . . The nest was of dry leaves, about 11 inches deep and seven inches in diameter."

One animal "was shot from the large hollow in the side of a giant 'ligaron' [higaron?] tree. This tree was fully 12 feet in diameter at waist height, and contained a hollow about 60 feet high and five feet in diameter."
Dalquest (1953, p. 19; eastern San Luis Potosí, MÉXICO)

"A burrow near Huichihuayan was in muddy soil beneath a clump of thorny bamboo, and another was beneath a decayed log, nearly concealed by vines and succulent plants. This burrow was so shallow that when the log was rolled aside the entire burrow was exposed. A nest at the end of the branched burrow consisted of a formless mass of dry leaves a foot in diameter. Two feet away there was an opening to the dense vegetation beside the log. Three feet farther on, the burrow emerged from under the log; and a trough like trail led through the vegetation to the river, twenty feet away."

\section{Murie (1935, p. 16; GUATEMALA)}

"In the edges of the pine ridge, especially near the streams, were numerous well worn trails about five inches wide, which were apparently made by these opossums. Many of the trails led from one ground burrow to another. At the end of one of the burrows about fifteen inches from the entrance, I found a nest ten inches in diameter, composed of dry sedges."

\section{Enders (1966, p. 201; PANAMÁ)}

After the young are born the female moves about more. Unlike habits during the rest of the year (Enders, 1935, p. 397), they frequent the same nest. Later, when the young are able to detach themselves and so leave the mother, young may continue to use the nest.

\section{Husson (1978, p. 26; SURINAME)}

Nest with female and seven young found under a tree. Nothing more is said.

\section{Miles et al. (1981a, p. 341; BRASIL)}

"Nests of the opossum were often found 8-10 $\mathrm{m}$ above the forest floor in hollow trees or as open nests in tree forks; some nests were, however, terrestrial, in cavities besides buttressed tree roots."

\section{Farris (1950, p. 259; PANAMÁ)}

"The animals usually were found in their nests in palm trees, at the branching and in the leaves about 5 feet off the ground where conditions were relatively dry." 


\section{Population Density}

Charles-Dominique et al. (1981. p. 383; Cayenne, GUYANE FRANÇAISE)

Philander opossum was one of the most abundant mammals on the island. Its biomass, based on 199 observations within a square kilometer. was calculated as between 100 and 200 individuals, with a biomass between 40 and 60 kilograms in the same area. The biomass of each of the five other marsupials of the same area was 25 to 50 $\mathrm{kg}$ for D. marsupialis, 30 to $60 \mathrm{~kg}$ for $C$. philander, 2 to $8 \mathrm{~kg}$ for Marmosa cinerea [Micoureus demerarae], and 0.7 to 3.5 for $M$. murina.

Of the six marsupial species inhabiting the island, Philander, Didelphis, Caluromys, and $M$. murina were most abundant. Marmosa cinerea [= Micoureus demerarae] were moderately abundant: Monodelphis brevicaudata, uncommon.

Cerqueira et al. (1993, p. 513; Restinga de Barra de Maricá, Rio de Janeiro, 22 $57^{\circ} \mathrm{S}, 42^{\circ} 5 \mathrm{I}^{\prime} \mathrm{W}$, an impoverished small animal community, Atlantic forest, BRASIL)

Average density $1.91 / \mathrm{ha}$; population maintained mostly by recruitment.

Atramentowicz (1986b, p. 144; GUYANE FRANÇAISE)

Density per square kilometer based on observations from September 1978 to October 1979 was 137 P. opossum, $143 C$. philander, and $45 D$. marsupialis.

\section{Population Dynamics}

\section{Atramentowicz (1986b, GUYANE FRANÇAISE)}

"Five species of Didelphid marsupials were trapped live, marked, and released in a secondary forest in French Guiana during a 26-mo field study. Data on body weight, body length, dental stage, and reproductive state of females were collected. A total of 851 individuals, including 372 pouch-young, were monitored in 2273 captures. This abundance of data we made led us to a comparative analysis of the population dynamics of three species: Didelphis marsupialis, Philander opossum, and Caluromys philander, which represented 94 percent of the whole captures (Table 19). Some data on the two other species, Marmosa murina and $M$ [icoureus]. cinerea, were add- ed for comparison. These sympatric Didelphids are nocturnal, with a basically frugivorous and insectivorous diet. The three main species have high densities, high reproductive rates, short life-spans, and rapid population turnover. There are some differences in these parameters, mainly between the arboreal species, $C$. philander, and both terrestrial species, D. marsupialis and $P$. opossum. Although all have few social interactions and show a lack of territoriality, C. philander appear to have a longer life-span, a lower reproductive rate and a more sedentary population. $M$. cinerea [Micoureus demerarae] were very rare in this area, whereas M[armosa]. murina show large density variation."

The well-known tendency of American marsupials to enter baited traps, usually the same ones, time and again ensures a high degree of accuracy in estimating their duration of residence in an area. In general, both sexes of $P$. and $C$, philander are highly sedentary. Didelphis marsupialis, on the other hand, tends to wander, particularly the males (Table 19).

\section{Posture}

Enders (1935, p. 412; Canal Zone, PANAMÁ)

Philander "used the tripod [sitting] posture more frequently than Metachirus although the tail of the latter is a very effective appendage."

Hershkovitz (1941, field notes; northern COLOMBIA)

Sitting was tripodal, the body supported by hind limbs and tail.

\section{Sleep}

Temminck (1827, p. 38)

It sleeps during the day rolled up into a ball. Its breathing is like that of a ferret, and it leaves its hiding place only at night.

Schomburgk (1840, 348; GUYANA)

They sleep during the day under tree roots or in hollow trees and hunt at night.

Hershkovitz (1941, field notes; northern COLOMBIA)

The animal sleeps rolled up on its side or ex- 
tended backside up. The closed eyes are not visible, but the whitish supraorbital spots give the animal an appearance of being awake with eyes widely opened.

\section{Sociability}

Hall and Dalquest (1963, p. 179; Veracruz, MÉXICO)

"On a few occasions, two four-eyed opossums were seen as close together as 50 feet, but otherwise they were solitary."

Charles-Dominique (1983, pp. 405-407; GUYANE FRANÇAISE)

"Didelphids are always solitary day and night. During development immediately after the young have released the nipples, they remain for a short period in a nest ( 8 to 15 days for $P$. opossum and 30 to 45 days for $C[$ aluromys]. philander). During the first days (the first weeks for $C$. philander), the young maintain a certain cohesion between themselves (they often grasp each other) and explore the surroundings of the nest. This gregarious behavior indicates the persistence of a need for body contact, directed indiscriminately towards the litter mates as well as towards the mother. Sometimes one or several young of the litter cling to the mother's fur during nocturnal activity. . . . Individual recognition may not exist between lactating females and young. In the middle of the night, and again at dawn, the mother returns to the nest for 2 to $3 \mathrm{~h}$. This cohesive behavior disappears after weaning, mother and young becoming indifferrent or aggressive when they meet.

"We observed no durable social bonds between adult individuals. When two animals met (for example, in a fruiting tree), they often threatened each other with an aggressive vocalization and then continued their individual activities."

Charles-Dominique (1983, pp. 406, 407; Cayenne, GUYANE FRANÇAISE)

A dominance hierarchy or peck order does not exist among didelphoids. "Nineteen adult foureyed opossums (10 females; 9 males) habitually fed on a $1 \mathrm{~m}$ high platform baited daily with bananas. Animals were identified by collars (radiotransmitters and color bands) and only had access to the platform via a single branch. Observations under weak lighting were made from a distance 
of $3 \mathrm{~m}$. Conflicts were observed principally during the first $2 \mathrm{hr}$ of the night when the four-eyed opossums came to take the first meal ( 12 evenings of observation). Usually an animal which had not eaten chased one which was already eating on the platform. I observed direct fighting (bites) on only three occasions, but usually only threats were exchanged (hissing, open mouth. and start of chase). No rank order was observed and an individual (male or female) chased by another (male or female) one night could chase it in turn the following night depending on the situation (degree of hunger)."

\section{Spontaneous Bleeding}

Hershkovitz (1941, field notes; northern COLOMBIA)

An adult female $P$. opossum was clubbed by a woodsman and seized as she jumped from its nest in the felled tree. The animal was brought live to my camp, where it recovered without signs of injury. When irritated, however, it bled spontaneously from the tips of the fingers, the tail, and the nose. I had never witnessed or known of this phenomenon before.

\section{Stress}

Hunsaker and Shupe (1977, p. 302)

"Tight coiling of the tail . . occurs in Philander opossum, Didelphis marsupialis and Caluromys as a function of stress. It occurs when an animal is frightened and can be considered a submission posture."

The coiled tail reduces exposure from seizure by a predator.

\section{Tail Transport}

Hunsaker and Shupe (1977, p. 301)

"Tail coiling has been observed to facilitate carrying nesting material in Didelphis virginiana, Monodelphis domestica, Caluromys derbianus and Marmosa robinsoni. The fact that more species have not been observed to do so is probably a function of inadequate observations." Philander does coil its tail in the manner described and may indeed do so for transport of building material.
According to Layne (1951, p. 464), use of the tail for transport by Didelphis was first described and illustrated by Pray (1921, p. 109). Layne further elaborated on the subject.

\section{Taming}

Hershkovitz (1941, field notes; northern COLOMBIA)

The opossum captured alive after being struck with a machete by a woodsman was leashed and brought to me. It objected to being handled, was suspicious of anyone's approach, and reacted like all other didelphoids I've known by hissing with the mouth open. Within a few days, however, the animal became tame and permitted itself to be handled and stroked and seemed to enjoy climbing onto my shoulder and head.

\section{Thermoregulation}

Enders and Davis (1936, p. 165; Canal Zone, PANAMÁ)

Body 35.4, environment 26.1 (afternoon).

Miller (in J. A. Allen, 1916c, p. 589; Mato Grosso, BRASIL)

"As a general rule opossums cannot stand great heat and will soon die if left exposed to the direct rays of the tropical sun."

\section{Vocalization}

Charles-Dominique (1983, pp. 407, 411, 412; Cayenne, GUYANE FRANÇAISE)

"Two types of calls by adults are the weak sexual call (rarely by the male) and an agressive call by both sexes.

"The young begin emitting very short highpitched "clicks" about one month before releasing the nipples, especially when the mother licks the young attached to the nipples in her pouch, or when the young are experimentally pulled off the nipples.

"These clicks progressively disappear when the young release the nipple and the first 'hiss'-associated with the typical posture (opened mouth)-is emitted very early in conditions of fear or when pulled off the nipple." 
In the case of $C$. philander the clicking sound was particularly loud, audible at $200 \mathrm{~m}$. The same sound, but muted, was emitted by Marmosa murina, Monodelphis brevicaudata, and D. marsupialis.

The clicking sound has also been recorded by Reynolds (1952, p. 235) for D. virginiana as being most pronounced during breeding. Kirsh (1979, p. 392) heard the sound from Caenolestes fuliginosus; Thrasher et al. (1971), from Marmosa robinsoni. Bruce Patterson (personal communication) heard Metachirus nudicaudatus clicking as a signal of aggression.

Apparently all New World marsupials are capable of three types of calls: the standard hiss, usually uttered when standing erect or tripodally in an aggressive posture, is usually fear stimulated; a mouselike squeak, which may be a sexual call; and the click, which is emitted under various conditions.

\section{Weight}

Tyndale-Biscoe $(1980 ; P$. opossum-COLOMBIA)

Weights of adults (g): $8 \delta \delta, 416.9 \pm 132.1$ (230-675); 4 우우, $323.8 \pm 65.2$ (275-420).

\section{Alphabetical List of South American Collecting Localities of Philander with Key Numbers to Distribution Map (Fig. 22) and Gazetteer (p. 85)}

See Hall and Kelson (1959) for Middle American portion of range.

Acanaña; Territorio Federal Amazonas, Venezuela (107)

Agua Limpa; Brasília, Distrito Federal, Brasil (175c)

Agua Viva; Trujillo, Venezuela (98a)

Albina; Marowijne, Suriname (128)

Além Paraíba; Minas Gerais, Brasil (182)

Alguacil; Zulia, Venezuela (97a)

Altamira; Pará, Brasil (151)

Alto Bonito; Antioquia, Colombia (1)

Alto da Serra; São Paulo, Brasil (202)

Ananindéua; Pará, Brasil (159)

Anapolis; Goiás, Brasil (174b)
Apayacu; Ucayali, Perú (43)

Aragarças; Goiás, Brasil (173)

Arruda; El Beni, Bolivia (73)

Arumateua; Pará, Brasil (157)

Ascención de Guarayos; Santa Cruz, Bolivia (84)

Asunción; Central, Paraguay (9l)

Auará; Igarapé; Amazonas, Brasil (144)

Avanavero Falls; Nickerie, Suriname (119)

Avanhandava (Lajeado); São Paulo, Brasil (195)

Ayacucho (Ibáñez); Santa Cruz, Bolivia (88)

Bacaetava; São Paulo, Brasil (199)

Bagadó; Chocó, Colombia (4)

Bahia; Bahia, Brasil (172)

Baião; Pará, Brasil (156)

Balisa; Mato Grosso, Brasil (168d)

Balta; Ucayali, Perú (55)

Barra do Garças; Mato Grosso, Brasil (168c)

Barranquita; El Beni, Bolivia (73)

Barrieira; Rio de Janeiro, Brasil (189)

Barro Branco; Rio de Janeiro, Brasil (193)

Baptista (Lago); Amazonas, Brasil (141)

Belém; Pará, Brasil (159)

Belém-Brasília (Rodovia); Pará, Brasil (159)

Belén; Territorio Federal Amazonas, Venezuela (106)

Bellavista; Antioquia, Colombia (2)

Bemberg (Puerto); Misiones, Argentina (95)

Benfica; Minas Gerais, Brasil (181)

Better Hope; East Demerara, Guyana (not located but likely near Georgetown [114] and perhaps same as Hope $\left.\left[6^{\circ} 42^{\prime} \mathrm{N}, 57^{\circ} 57^{\prime} \mathrm{W}\right]\right)$

Boa Esperança; Minas Gerais, Brasil (179)

Boa Fé (Fazenda); Rio de Janeiro, Brasil (189)

Boa Vista (Fazenda); Rio de Janeiro, Brasil (189)

Boa Vista; Roraima, Brasil (134)

Boca Colorado; Madre de Dios, Perú (65)

Bom Jardim; Brasil; not located; many so-named throughout most states

Boracéia (Ponta); São Paulo, Brasil (203)

Boracéia, Rio Tietê; São Paulo, Brasil (196)

Branco (Rio); Amapá, Brasil (133)

Brasília; Distrito Federal, Brasil (175c)

Bucay; Guayas, Ecuador (28)

Buck Hall; Essequibo Islands-West Demerara, Guyana (116)

Buenaventura; Valle del Cauca, Colombia (10)

Buenavista; Santa Cruz, Bolivia (86)

Butantã; São Paulo, Brasil (200)

Cáceres; Mato Grosso, Brasil (168a)

Cachaví (= Cachabí); Esmeraldas, Ecuador (18)

Cadena (Hacienda); Cuzco, Perú (63)

Caixa D'agua; Espírito Santo, Brasil (186)

Cali; Valle del Cauca, Colombia (11)

Cametá; Pará, Brasil (153) 


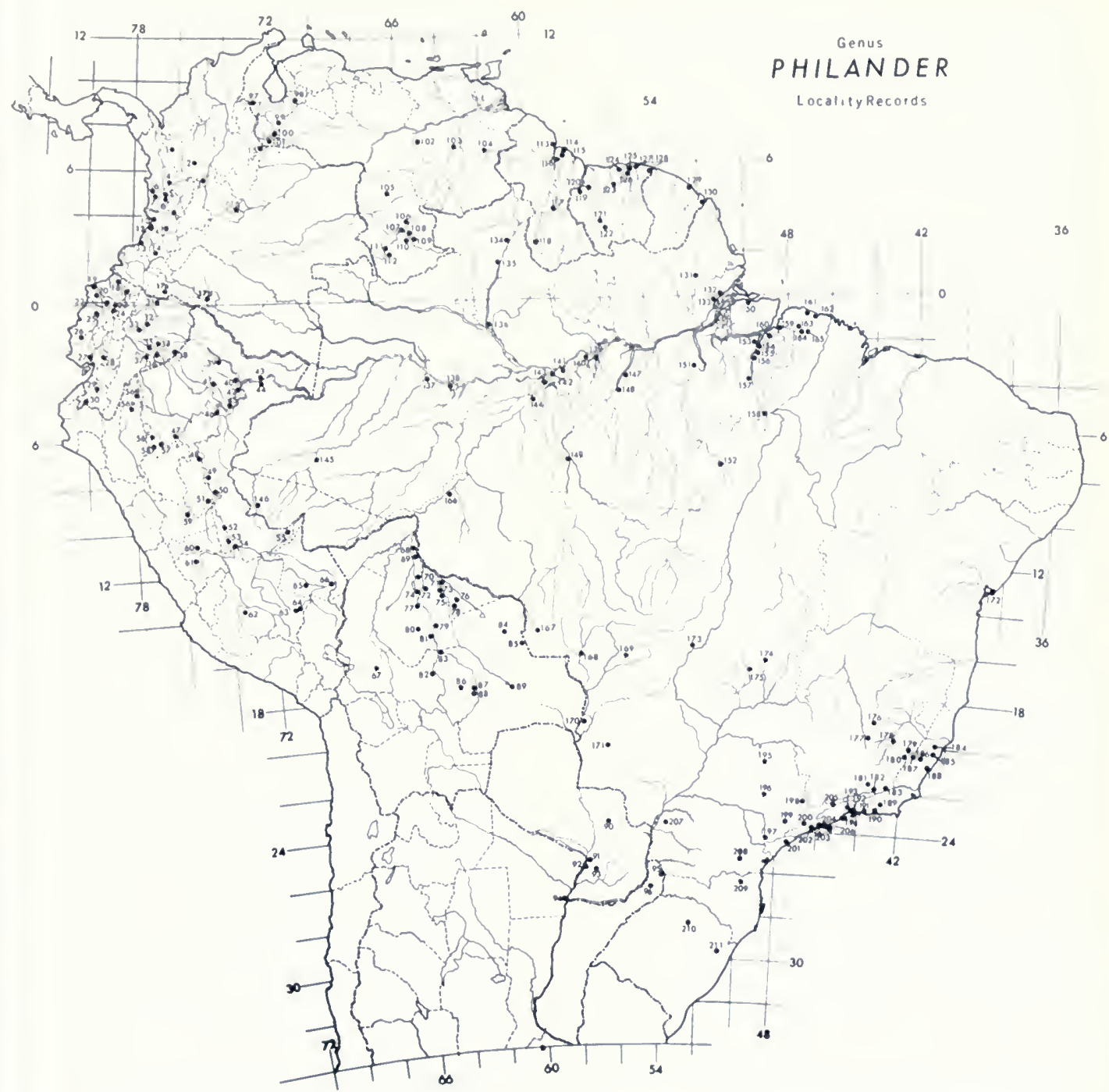

FIG. 22. South American collecting localities of Philander species. See Gazetteer (p. 85) for numbered locality names.

Camiaco; El Beni, Bolivia (81)

Camino Vilches; El Beni, Bolivia (73)

Campinho; Espírito Santo, Brasil (185)

Caney; Meta, Colombia (16)

Cantareira (Serra da); São Paulo, Brasil (200)

Canudos; Pará, Brasil (149)

Caoní (Río); Pichincha, Ecuador (22)

Capibara; Territorio Federal Amazonas, Venezuela (111)

Capim; Pará, Brasil (164)

Caracaraí; Roraima, Brasil (135)
Cardosa (Fazenda); Minas Gerais, Brasil (180)

Carondelet; Esmeraldas, Ecuador (18)

Casa Grande; São Paulo, Brasil (203)

Catatumbo (Puerto); Zulia, Venezuela (97b)

Cauca (Río); Valle del Cauca, Colombia (11)

Cayenne: Cayenne, Guyane Française (129)

Cebollal; Loja, Ecuador (30)

Cerro Cucurito; Territorio Federal Amazonas, Venezuela (109b)

Centinela; El Beni, Bolivia (73)

Chaco; Argentina (94) 
Chalimana; Bolívar, Venezuela (104c)

Chanchamayo; Junín, Perú (61)

Chaparé (Río); Santa Cruz, Bolivia (83)

Chicosa; Ucayali, Perú (53)

Chimbo (Puente); Guayas, Ecuador (28)

Chimbo-Coco (Ríos); Guayas, Ecuador (28)

Chocolatal, Monte, El Beni, Bolivia (75)

Chulumani; La Paz, Bolivia (67)

Churulí; Zulia, Venezuela (97a)

Clevia; Suriname, Suriname (125)

Cocal; Cauca, Colombia (14)

Coco (Río); Guayas, Ecuador (28)

Colônia do Prata; Pará, Brasil (163)

Colônia Hansa; Santa Catarina, Brasil (209)

Colorado (Boca); Madre de Dios, Perú (65)

Colorado (Río); Madre de Dios, Perú (65)

Commewijne (River); Commewijne, Suriname (127)

Comochatibá (= Comoxatibá) $17^{\circ} 06^{\prime} \mathrm{S}, 39^{\circ} 11^{\prime} \mathrm{W}$.

Maximilian Wied-Neuwied, July 1816.

Conceição do Mato Dentro; Minas Gerais, Brasil (176)

Condoto; Chocó, Colombia (5)

Copataza (Río); Pastaza, Ecuador (37)

Corumbá; Mato Grosso do Sul, Brasil (170)

Costão dos Engenhos; São Paulo, Brasil (201)

Cotia; São Paulo, Brasil (200)

Covaría (Río); Boyacá, Colombia (15)

Cucurito (Cerro); Territorio Federal Amazonas, Venezuela (109b)

Culturutuin; Suriname, Suriname (125)

Culumani; La Paz, Bolivia (67)

Cumaría; Ucayali, Perú (52)

Cumería; Ucayali, Perú (52)

Cumuruxotibá (= Comoxatibá = Comochatibá q.v.)

Curaray (Río); Napo, Ecuador (39)

Curaray (Boca); Ucayali, Perú (39)

De Oro (Río); Chaco, Argentina (94)

De Oro (Río); Zulia, Venezuela (97)

Dividive; Trujillo, Venezuela (98a)

Docampadó (Río); Chocó, Colombia (7)

Duida (Cerro); Territorio Federal Amazonas, Venezuela (108)

El Capricho (Finca); 38 km E Villavicencio, Meta, Colombia (16)

El Carmen; El Beni, Bolivia (78)

El Chiral; El Oro, Ecuador (29)

El Dividive; Trujillo, Venezuela (98a)

El Palmar; Bolívar, Venezuela (102a)

El Palmar; Santa Cruz, Bolivia (88)

Encontrados, Río de Oro; Zulia, Venezuela (97b)

Engenheiro Reeve; Espírito Santo, Brasil (188)

Engenheiro Rive (see Engenheiro Reeve)
Esmeralda (Fazenda); Minas Gerais, Brasil (178)

Esmeralda; Territorio Federal Amazonas, Venezuela (109a)

Esmeraldas; Esmeraldas, Ecuador (19)

Estrada de Santarém-Cuiabá; Pará, Brasil (148)

Exaltación; El Beni, Bolivia (74)

Fazenda Bõa Fé; Rio de Janeiro, Brasil (189)

Fazenda Cardosa; Minas Gerais, Brasil (180)

Fazenda da Floresta; Minas Gerais, Brasil (178)

Flor do Prado; Pará, Brasil (161)

Floresta (Fazenda); Minas Gerais, Brasil (178)

Fordlandia; Pará, Brasil (147)

Formosa; Goiás, Brasil (174a)

Fracrán; Misiones, Argentina (96)

Georgetown; Demerara-Mahaica, Guyana (114)

Gradaús; Pará, Brasil (152)

Gualea; Pichincha, Ecuador (23)

Guamá; Pará, Brasil (165)

Guaquitas; Barinas, Venezuela (100b)

Guayaquil; Guayas, Ecuador (27)

Guayaramarín; El Beni, Bolivia (68)

Güiniquina, Territorio Federal Delta Amacuro, Venezuela (98d)

Hacienda Cadena; Cuzco, Perú (63)

Hamacas; Santa Cruz, Bolivia (88)

Hansa; Santa Catarina, Brasil (209)

Hato San José; Bolívar, Venezuela (103)

Hondo (Río); Caldas, Colombia (3)

Huampami; Amazonas, Perú (56b)

Huanhuachayo; Ayacucho, Perú (62)

Hyde Park; Demerara-Mahaica, Guyana (115)

Ibáñez; Santa Cruz, Bolivia (88)

Ibaré (Boca); El Beni, Bolivia (79)

Ichilo (Río); Cochabamba, Bolivia (82)

Igarapé Açu; Pará, Brasil (163)

Igarapé Tapereba; Pará, Brasil (150)

Ilha do Taiuna; Pará, Brasil (153)

Imperatriz; Amazonas, Brasil (140)

Inañez; Pasco, Perú (not located)

"High ground between Chanchamayo and up-

per Río Pachitea" (Hendee in Thomas, 1928a, Annals and Magazine of Natural History, Series 10, 2:250).

R. W. Hendee, June 1927, at $5000 \mathrm{ft}$

Ipanema; São Paulo, Brasil (199)

1peau-Apez; Pará, Brasil (159)

Ipitinga; Pará, Brasil (160)

lpixuna; Amazonas, Brasil (138)

lporanga; São Paulo, Brasil (197)

Iquitos; Ucayali, Perú (42)

Iriteria; Pará, Brasil (165)

Itaituba; Pará, Brasil (148)

Itatiaia (Parque Nacional de); Rio de Janeiro, Brasil (193) 
Itatiaia (Serra de); São Paulo, Brasil (204)

Itaya; Ucayali, Perú (42)

Itonama; El Beni, Bolivia (71)

Joinville; Santa Catarina, Brasil (209)

Juiz de Fora; Minas Gerais, Brasil (183)

Juquia: São Paulo, Brasil (201)

Kaiserberg Airstrip; Nickerie, Suriname (122)

Kanuku Mountains; Upper Takutu-Upper Essequibo, Guyana (118)

Km 19: Pará, Brasil (148)

Km 90; Pará, Brasil (159)

Km 216; Pará, Brasil (148)

La Blanquita; Apure, Venezuela (101a)

La Boca, Río Saijá; Cauca, Colombia (13)

La Lengueta; Barinas, Venezuela $(100 \mathrm{c})$

La Neblina; Territorio Federal Amazonas. Venezuela (112b)

La Papaya; Manabí, Ecuador (not located)

T. Mena, May 1942, at $50 \mathrm{~m}$

La Ponchera: Táchira, Venezuela (10lb)

La Poule: Saramacca, Suriname (124)

La Poza; Amazonas, Perú (56a)

Lagarto Cocha: Ucayali, Perú (54)

Lago do Baptista; Amazonas, Brasil (141)

Lago Mirañes; Ucayali, Perú (40)

Lagoa Santa; Minas Gerais, Brasil (177)

Lapango; Central, Paraguay (92)

Las Bonitas; Barinas, Venezuela (100b)

Lazaropolis do Prata; Pará, Brasil (not located)

Lelydorp; Suriname, Suriname (126)

Lelydorpplan; Suriname, Suriname (126)

Limón; Ecuador (not located)

G. H. H. Tate, December 1923, at $1200 \mathrm{ft}$

Loksie Hattie; Brokopondo, Suriname (123)

Los Guires, Territorio Federal Delta Amacuro, Venezuela (98c)

Macapá; Amapá, Brasil (132)

Macieiras; Rio de Janeiro, Brasil (193)

Madeira (Rio); Amazonas, Brasil (142)

Magdalena; El Beni, Bolivia (76)

Majagua (Caño); Territorio Federal Amazonas, Venezuela (105)

Makerie; Nickerie, Suriname (121)

Maldonado (Puerto); Madre de Dios, Perú (66)

Mamoré (Rio); El Beni, Bolivia (70)

Mamoré (Rio); Santa Cruz, Bolivia (83)

Mangaratiba; Rio de Janeiro, Brasil (192)

Marabá; Pará, Brasil (158)

Marco; Pará, Brasil (159)

Maripa; Bolívar, Venezuela (102b)

Matipó (Rio); Minas Gerais, Brasil (178)

Mato Grosso; Mato Grosso, Brasil (167)

Mavaca; Territorio Federal Amazonas, Venezuela $(110 c)$
Mazagão; Amapá, Brasil (133)

Mazagão; Pará. Brasil (154)

Mazán (Río); Ucayali, Perú (40)

Mercedes; Santa Cruz, Bolivia (85)

Merey: Territorio Federal Amazonas, Venezuela (112a)

Mérida; Mérida, Venezuela (99)

Merredor; El Beni, Bolivia (69)

Mindo; Pichincha, Ecuador (24)

Miranda; Mato Grosso do Sul, Brasil (171a)

Mirañes (Lago); Ucayali, Perú (40)

Mocajuba; Pará, Brasil (155)

Montalvo: Pastaza, Ecuador (36)

Monte Alegre; São Paulo, Brasil (198)

Monte Alegre; Mato Grosso, Brasil ( 169)

Monte Chocolatal (see San Ramón); El Beni, Bolivia (75)

Monte Serrat; Rio de Janeiro, Brasil (193)

Montes Claras; Minas Gerais, Brasil (178)

Motatán, $1 \mathrm{~km}$ E and $5 \mathrm{~km}$ NNE; Trujillo, Venezuela (98a)

Motatán, Rio; Trujillo, Venezuela (98a)

Moyobamba; San Martín, Perú (57)

Murutucu; Pará, Brasil (159)

Napo (Puerto); Napo, Ecuador (33)

Napo (Río); Napo, Ecuador (33)

Nauta; Ucayali, Perú (45)

Navio (Serra do); Amapá, Brasil (131)

Nickerie (River); Nickerie, Suriname (120)

Nóvita (Río), Chocó, Colombia (8)

Novo Friburgo; Rio de Janeiro, Brasil (189)

Nulita; Apure, Venezuela (101a)

Órgãos (Serra dos); Rio de Janeiro, Brasil (189)

Oro (Río de); Chaco, Argentina (94)

Orosa; Ucayali, Perú (44)

Ouanary (River); Ouanary, Guyane Française (130)

Oxapampa; Pasco, Perú (60)

Pacific Virology Field Camp; Valle del Cauca, Colombia (12)

Palacios; El Beni, Bolivia (74)

Palmeiras; Mato Grosso. Brasil (169)

Papelón (Cerro); Monagas, Venezuela (98b)

Pará; Pará, Brasil (159)

Pará (River); Suriname, Suriname (125)

Paracaí (Rio); Paraná, Brasil (207)

Paramaribo; Suriname, Suriname (125)

Paramba; Imbabura, Ecuador (21)

Parima (Sierra); Territorio Federal Amazonas, Venezuela $(110 \mathrm{~b})$

Parintins; Amazonas, Brasil (139)

Parintins (Serra de); Amazonas, Brasil (139)

Parque Nacional de Brasília; Distrito Federal, Brasil (175b) 
Parque Nacional de Itatiaia; Rio de Janeiro, Brasil (193)

Passo Fundo; Rio Grande do Sul, Brasil (210)

Pedra Branco; Rio de Janeiro, Brasil (194)

Peixe-Boi; Pará, Brasil (163)

Pescado (Río); Manabí, Ecuador (25)

Piñas; El Oro, Ecuador (29)

Pindo Yacu (Río); Pastaza, Ecuador (38)

Piquete; São Paulo, Brasil (205)

Playa del Río Base; Territorio Federal Amazonas, Venezuela (108)

Pocão, Bõa Vista; Roraima, Brasil (134)

Pocão, Caracaraí; Roraima, Brasil (135)

Poconé; Mato Grosso, Brasil (168b)

Ponta de Boracéia; São Paulo, Brasil (203)

Porto Velho; Rondônia, Brasil (166)

Pucallpa; Ucayali, Perú (51)

Puente de Chimbo; Guayas, Ecuador (28)

Puerto Asis; Putumayo, Colombia (17a)

Puerto Bemberg; Misiones, Argentina (95)

Puerto Caballo; El Beni, Bolivia (74)

Puerto Catatumbo; Zulia, Venezuela (97b)

Puerto Maldonado; Madre de Dios, Perú (66)

Puerto Napo; Napo, Ecuador (33)

Puerto Siles; El Beni, Bolivia (72)

Punta Santa Ana; Loja, Ecuador (29)

Quartel de Sacramento; Minas Gerais, Brasil (178)

Quincemil; Cuzco, Perú (64)

Quinindé (Río); Esmeraldas, Ecuador (20)

Raposo (Río); Valle del Cauca, Colombia (12)

Restrepo; Meta, Colombia (16)

Rijweg; Suriname, Suriname (125)

Río de Janeiro; Rio de Janeiro, Brasil (190)

Río de Oro; Chaco, Argentina (94)

Río de Oro; Manabí, Ecuador (26)

Río de Oro; Zulia, Venezuela (97b)

Río Frío; Valle del Cauca, Colombia (9)

Río Hondo; Caldas, Colombia (3)

Rioja; San Martín, Perú (58)

Roça Nova; Paraná, Brasil (208)

Rodeio; Rio de Janeiro, Brasil (191)

Rodovia Belém-Brasília; Pará, Brasil (159)

Rosarinho; Amazonas, Brasil (143)

Rupununi (River); Rupununi, Guyana (117)

Saijá (Río); Cauca, Colombia (13)

Salesopolis; São Paulo, Brasil (203)

Salobra; Mato Grosso do Sul, Brasil (171b)

Salvador; Bahia, Brasil (172)

Salvias; El Oro, Ecuador (29)

San Ignacio de Móxos; El Beni, Bolivia (80)

San Javier; Esmeraldas, Ecuador (18)

San Jerónimo; Cuzco, Perú (63)

San Jerónimo; Ucayali, Perú (49)
San Joaquín; ElBeni, Bolivia (73)

San José; Ayacucho, Perú (62)

San José; (Hato); Bolívar, Venezuela (103)

San José, Abajo; Napo, Ecuador (32)

San Juan; Territorio Federal Amazonas, Venezuela (105)

San Martín de Turumbán; Bolívar, Venezuela (104a)

San Pablo; El Beni, Bolivia (77)

San Ramón; El Beni, Bolivia (75)

San Ramón; Santa Cruz, Bolivia (89)

Sandó (Río); Chocó, Colombia (6)

Santa Ana, Punta; Loja, Ecuador (29)

Santa Cecilia; Napo, Ecuador (31)

Santa Clara; Amazonas, Brasil (140)

Santa Cruz de la Sierra; Santa Cruz, Bolivia (88)

Santa Elena; Ucayali, Perú (46)

Santa Luisa; Ucayali, Perú (41)

Santa María; Pará, Brasil (162)

Santa Rosa; El Beni, Bolivia (72)

Santa Rosa; Ucayali, Perú (54)

Santa Rosita; Santa Cruz, Bolivia (87)

Santa Teresa; Espírito Santo, Brasil (184)

Santa Teresa; Mato Grosso do Sul, Brasil (170)

Santo Antônio de Uayara; Amazonas, Brasil (145a)

Santo Dios; El Beni, Bolivia (73)

Santo Isidoro; Amazonas, Brasil (137)

Santarém; Pará, Brasil (148)

Santarém-Cuiabá (Estrada); Pará, Brasil (148)

São João de Petrópolis; Espírito Santo, Brasil (184)

São João Marcos; Rio de Janeiro, Brasil (191)

São Luis de Cáceres; Mato Grosso, Brasil (168a)

São Miguel do Guamá; Pará, Brasil (165)

São Paulo; São Paulo, Brasil (200)

São Sebastião; São Paulo, Brasil (206)

Sapitiba (see Sepetiba); Rio de Janeiro, Brasil (191)

Sapucaí; Paraquari, Paraguay (93)

Sapucajuba; Pará, Brasil (159)

Sapucay; Paraguarí, Paraguay (93)

Sarayacu; Pastaza, Ecuador (35)

Sarayacu; Ucayali, Perú (48)

Seboyal; Loja, Ecuador (30)

Sepetiba; Rio de Janeiro, Brasil (191)

Seringal Oriente; Acre, Brasil (146)

Serra; Espírito Santo, Brasil (185)

Serra de Itatiaia; São Paulo, Brasil (204)

Serra de Parintins; Amazonas, Brasil (139)

Serra do Navio; Amapá, Brasil (131)

Serra dos Órgãos; Rio de Janeiro, Brasil (189)

Serrat Monte; Rio de Janeiro, Brasil (193) 
“S. Francisco de C." Minas Gerais, Brasil (not located)

A. Robert, March 1900, at $1580 \mathrm{~m}$.

Shahuía; Ucayali, Perú (52)

Siles (Puerto); El Beni, Bolivia (72)

Sitio Minas Gerais; Minas Gerais, Brasil (not located)

Supenam (River) (see Supinaam River); Demerara-Mahaica, Guyana (113)

Supinaam (River); Demerara-Mahaica, Guyana (113)

Tacuati; San Pedro, Paraguay (90)

Taiuana (Ilha); Pará, Brasil (153)

Tamatama; Territorio Federal Amazonas, Venezuela (110a)

Tapereba (Igarapé); Pará, Brasil (150)

Taquara; Rio Grande do Sul, Brasil (211)

Tefé; Amazonas, Brasil (137)

Teresinha; Amapá, Brasil (131)

Teresópolis; Rio de Janeiro, Brasil (189)

Terezinha (see Teresinha); Amapá, Brasil (131)

Theresopolis (see Teresópolis); Rio de Janeiro, Brasil (189)

Ticoporo, Reserva Forestal; Barinas, Venezuela (100a)

Tingo María; Huánuco, Perú (59)

Tobesobe, Guayo; Territorio Federal Delta Amacuro, Venezuela (98c)

Tocomechi; Santa Cruz, Bolivia (88)

Tres Troncos; Caquetá, Colombia (17b)

Trinidade; Goiás, Brasil (175a)

Turumbán (= San Martín de Turumbán); Bolivar, Venezuela (104a)

Uragua-i (Río); Misiones, Argentina (95)

Urubamba (Río); Ucayali, Perú (54)

Urucu, Rio; Amazonas, Brasil (145b)

Urucum; Mato Grosso do Sul, Brasil (170)

Utcuyacu; Junín, Perú (61)

Utinga; Pará, Brasil (159)

Vaca Diez; El Beni, Bolivia (79)

Valão de São Lourenço; Espírito Santo, Brasil (187)

Ventura; Guayas, Ecuador (28)

Vila Oliveira; São Paulo, Brasil (202)

Vila Velha; Espírito Santo, Brasil (185)

Villa Bella Imperatriz; Amazonas, Brasil (140)

Villavicencio; Meta, Colombia (16)

Vitoria; Espírito Santo, Brasil (185)

Warnes; Santa Cruz, Bolivia (87)

Xiriviny (Rio); Amazonas, Brasil (136)

Yana Rumi (Río); Napo, Ecuador (34)

Yana Yacu; Ucayali, Perú (47)

Yarinacocha; Ucayali, Perú (50)

Ypanema (= Ipanema); São Paulo, Brasil (199)

Yurac Yacu; San Martín, Perú (56c)

Yurimaguas; Ucayali, Perú (47)
Yuruán (Río); Bolívar, Venezuela (104b).

\section{Gazetteer of South American Collecting Localities of Philander}

Colombia-Philander opossum fuscogriseus, $P$. opossum quica, P. andersoni andersoni

\section{Antioquia-Philander opossum fuscogriseus}

1. Alto Bonito, $7^{\circ} 01^{\prime} \mathrm{N}, 76^{\circ} 17^{\prime} \mathrm{W}$, about $400 \mathrm{~m}$, upper Río Sucio, W slope Cordillera Occidental.

L. E. Miller and H. S. Boyle, February 1915.

2. Bellavista, $6^{\circ} 33^{\prime} \mathrm{N}, 75^{\circ} 18^{\prime} \mathrm{W}$, upper Río Porce, Cordillera Centra.

P. Hershkovitz, February 1950, at $1200 \mathrm{~m}$.

\section{Caldas-Philander opossum fuscogriseus}

3. Río Hondo, Samaná, $5^{\circ} 42^{\prime} \mathrm{N}, 75^{\circ} 01^{\prime} \mathrm{W}$, Cordillera Central.

P. Hershkovitz, March 1951.

\section{Chocó-Philander opossum fuscogriseus}

4. Bagadó, $5^{\circ} 25^{\prime} \mathrm{N}, 76^{\circ} 24^{\prime} \mathrm{W}$, Río Andagueda. E. L. Kerr, November 1912, at $650 \mathrm{~m}$.

5. Condoto, $5^{\circ} 06^{\prime} \mathrm{N}, 76^{\circ} 37^{\prime} \mathrm{W}$, Río Condoto, tributary of Rio San Juan.

H. G. F. Spurrell, February 1914, at $92 \mathrm{~m}$.

6. Río Sandó, Río Baudó, $5^{\circ} 03^{\prime} \mathrm{N}, 76^{\circ} 57^{\prime} \mathrm{W}$.

K. von Sneidern, October 1958, at $160 \mathrm{~m}$.

7. Río Docampadó, $4^{\circ} 45^{\prime} \mathrm{N}, 77^{\circ} 18^{\prime} \mathrm{W}$.

K. von Sneidern, September 1958, at 75$160 \mathrm{~m}$.

8. Nóvita, $4^{\circ} 57^{\prime} \mathrm{N}, 76^{\circ} 34^{\prime} \mathrm{W}$, Río Tamaná.

L. E. Miller, December 1911, at $120 \mathrm{~m}$.

Valle del Cauca-Philander opossum fuscogriseus

9. Río Frío, $4^{\circ} 09^{\prime} \mathrm{N}, 76^{\circ} 15^{\prime} \mathrm{W}$, enters Río Cauca from east.

A. A. Allen and L. E. Miller, NovemberDecember 1911, at $100 \mathrm{~m}$.

10. Buenaventura, $3^{\circ} 53^{\prime} \mathrm{N}, 77^{\circ} 04^{\prime} \mathrm{W}$.

K. von Sneidern, February 1958, at near 
sea level.

C. H. Tyndale-Biscoe, September 1971.

10. Buenaventura, $28 \mathrm{~km} \mathrm{NE}$.

J. A. W. Kirsch, September 1969, at $150 \mathrm{~m}$.

11. Cali, $3^{\circ} 27^{\prime} \mathrm{N}, 76^{\circ} 31^{\prime} \mathrm{W}$, upper Río Cauca valley.

C. H. Tyndale-Biscoe, September 1971.

11. "Rio Cauca" (see Cali).

J. H. Batty, June 1898.

12. Raposo (Río), $3^{\circ} 43^{\prime} \mathrm{N}, 77^{\circ} 08^{\prime} \mathrm{W}$, Pacific Virology Field Camp.

J. Duran, June 1962, at near sea level.

Cauca-Philander opossum fuscogriseus

13. Río Saijá, La Boca (= Mouth), $2^{\circ} 52^{\prime} \mathrm{N}$, $77^{\circ} 41^{\prime} \mathrm{W}$.

K. von Sneidern, June 1958, at near sea level.

14. Cocal, $2^{\circ} 31^{\prime} \mathrm{N}, 77^{\circ} 00^{\prime} \mathrm{W}$, upper Río San Juan.

L. E. Miller, July 1911 , at 125 and $187 \mathrm{~m}$.

Boyacá-Philander opossum quica

15. Río Covaría, $7^{\circ} 03^{\prime} \mathrm{N}, 72^{\circ} 04^{\prime} \mathrm{W}$, near mouth, above town of Covaría.

K. von Sneidern, March 1959, at $350 \mathrm{~m}$.

\section{Meta-Philander opossum quica}

16. Caney, Río Guatiquía (see Restrepo).

16. Restrepo, $4^{\circ} 15^{\prime} \mathrm{N}, 73^{\circ} 33^{\prime} \mathrm{W}$.

16. Villavicencio, $4^{\circ} 09^{\prime} \mathrm{N}, 73^{\circ} 37^{\prime} \mathrm{W}$, upper Río Guatiquía.

R. Gilmore, February, May, June 1939, at $465 \mathrm{~m}$.

C. H. Tyndale-Biscoe, 1971.

16. El Capricho (Finca), $38 \mathrm{~km}$ E Villavicencio, $4^{\circ} 09^{\prime} \mathrm{N}, 73^{\circ} 16^{\prime} \mathrm{W}$.

J. A. W. Kirsch, October 1969.

Putumayo-Philander andersoni andersoni

17a. Puerto Asis, $17 \mathrm{~km} \mathrm{~N} ; 0^{\circ} 31^{\prime} \mathrm{N}, 76^{\circ} 31^{\prime} \mathrm{W}$. J. A. W. Kirsch, November 1969, at $330 \mathrm{~m}$.

Caquetá-Philander andersoni andersoni

17b. Tres Troncos, $0^{\circ} 08^{\prime} \mathrm{N}, 74^{\circ} 41^{\prime} \mathrm{W}$, above $\mathrm{La}$ Tagua, Río Caquetá.

P. Hershkovitz, January 1952, at $182 \mathrm{~m}$.
Ecuador-Philander opossum melanurus, $P$. opossum fuscogriseus, $P$. andersoni andersoni

Esmeraldas-Philander opossum melanurus

18. Cachaví (Cachabí), Río Cachaví, $1^{\circ} 03^{\prime} \mathrm{N}$, $78^{\circ} 50^{\prime} \mathrm{W}$.

18. Carondelet, $1^{\circ} 03^{\prime} \mathrm{N}, 78^{\circ} 50^{\prime} \mathrm{W}$.

G. Fleming, October 1900.

18. San Javier, $1^{\circ} 04^{\prime} \mathrm{N}, 78^{\circ} 47^{\prime} \mathrm{W}$.

G. Fleming, August 1900, at $20 \mathrm{~m}$.

19. Esmeraldas, $0^{\circ} 59^{\prime} \mathrm{N}, 79^{\circ} 42^{\prime} \mathrm{W}$.

W. Richardson, November 1912, at near sea level.

20. Río Quinindé, $0^{\circ} 20^{\prime} \mathrm{N}, 79^{\circ} 28^{\prime} \mathrm{W}$.

Imbabura-Philander opossum melanurus

21. Paramba, $0^{\circ} 49^{\prime} \mathrm{N}, 78^{\circ} 21^{\prime} \mathrm{W}, 1100 \mathrm{~m}$.

W. F. H. Rosenberg, April 1897; L. Gomez, June 1941.

Pichincha-Philander opossum melanurus

22. Río Caoní, $0^{\circ} 12^{\prime} \mathrm{N}, 79^{\circ} 23^{\prime} \mathrm{W}$.

M. Olalla, January 1935.

23. Gualea, $0^{\circ} 08^{\prime} \mathrm{N}, 78^{\circ} 48^{\prime} \mathrm{W}$, ca. $1250 \mathrm{~m}$.

L. Söderström, May 1920.

24. Mindo, below $8^{\circ} 02^{\prime} \mathrm{S}, 78^{\circ} 48^{\prime} \mathrm{W}$.

L. Söderström, December 1924, at 1000 m.

Manabí-Philander opossum fuscogriseus

25. Río Pescado, $1^{\circ} 25^{\prime} \mathrm{S}, 80^{\circ} 15^{\prime} \mathrm{W}$.

G. H. H. Tate, May 1922, at $500 \mathrm{~m}$.

26. Río de Oro, $2^{\circ} 10^{\prime} \mathrm{S}, 79^{\circ} 22^{\prime} \mathrm{W}$.

W. Richardson, January 1917.

Guayas-Philander opossum fuscogriseus

27. Guayaquil, $2^{\circ} 10^{\prime} \mathrm{S}, 79^{\circ} 50^{\prime} \mathrm{W}$, near sea level. E. Belcher, 1838 .

28. Puente de Chimbo, $2^{\circ} 10^{\prime} \mathrm{S}, 79^{\circ} 07^{\prime} \mathrm{W}$.

G. H. H. Tate, August 1922, at $375 \mathrm{~m}$.

28. Ríos Chimbo-Coco, $2^{\circ} 10^{\prime} \mathrm{S}, 79^{\circ} 50^{\prime} \mathrm{W}$.

G. H. H. Tate, July 1922 , at $750 \mathrm{~m}$.

28. Bucay, Río Chimbo, $2^{\circ} 10^{\prime} \mathrm{S}, 79^{\circ} 06^{\prime} \mathrm{W}$.

G. H. H. Tate, November 1921 , at $312 \mathrm{~m}$.

28. Ventura, Río Chanchán, $2^{\circ} 17^{\prime} \mathrm{S}, 79^{\circ} 24^{\prime} \mathrm{W}$.

G. H. H. Tate, April 1922, at $750 \mathrm{~m}$. 
El Oro-Philander opossum fuscogriseus

29. El Chiral, $2^{\circ} 39^{\prime} \mathrm{S}, 79^{\circ} 43^{\prime} \mathrm{W}$.

H. E. Anthony, August 1920, at $167 \mathrm{~m}$.

29. Piñas, $3^{\circ} 42^{\prime} \mathrm{S}, 79^{\circ} 42^{\prime} \mathrm{W}$.

G. H. H. Tate, September 1921, at $1125 \mathrm{~m}$.

29. Salvias, $3^{\circ} 47^{\prime} \mathrm{S}, 79^{\circ} 21^{\prime} \mathrm{W}$.

H. E. Anthony, August 1920, at $1000 \mathrm{~m}$.

Loja-Philander opossum fuscogriseus

29. Santa Ana, Punta (?) $3^{\circ} 50^{\prime} \mathrm{S}, 79^{\circ} 25^{\prime} \mathrm{W}$, on road from Zaruma to Loja.

H. E. Anthony, December 1920, at $114 \mathrm{~m}$.

30. Seboyal (see Cebollal).

30. Cebollal, $4^{\circ} 02^{\prime} \mathrm{S}, 80^{\circ} 02^{\prime} \mathrm{W}$.

G. H. H. Tate, October 1921, at 968 m.

Napo-Philander andersoni andersoni

31. Santa Cecilia, $0^{\circ} 03^{\prime} \mathrm{N}, 76^{\circ} 58^{\prime} \mathrm{W}$.

32. San José, abajo (= below), $0^{\circ} 31^{\prime} \mathrm{S}, 77^{\circ} 20^{\prime} \mathrm{W}$. Olalla Brothers, April 1924.

33. Puerto Naop, Río Napo, $1^{\circ} 03^{\prime} \mathrm{S}, 77^{\circ} 47^{\prime} \mathrm{W}$.

33. Río Napo (near) (see Puerto Napo).

L. Söderström, March 1921.

34. Río Yana Rumi, $1^{\circ} 38^{\prime} \mathrm{S}, 76^{\circ} 59^{\prime} \mathrm{W}$.

R. Olalla, October 1934.

Pastaza-Philander andersoni andersoni

35. Sarayacu, Río Bobonaza, $1^{\circ} 44^{\prime} \mathrm{S}, 77^{\circ} 29^{\prime} \mathrm{W}$.

G. H. H. Tate, March 1924, between 450 and $500 \mathrm{~m}$.

36. Montalvo, Río Bobonaza, $2^{\circ} 04^{\prime} \mathrm{S}, 76^{\circ} 58^{\prime} \mathrm{W}$.

R. Olalla, February 1932.

37. Río Copataza, $2^{\circ} 07^{\prime} \mathrm{S}, 77^{\circ} 27^{\prime} \mathrm{W}$.

R. Olalla, March 1939; G. H. H. Tate, March 1924.

38. Río Pindo Yacu, $2^{\circ} 08^{\prime} \mathrm{S}, 76^{\circ} 03^{\prime} \mathrm{W}$.

R. Olalla, October 1934, at $250 \mathrm{~m}$.

Perú-Philander opossum quica, P. andersoni andersoni. $P$. andersoni mcilhennyi

Ucayali-Philander opossum quica $(42,43,48$ $51,54,55), P$. andersoni andersoni $(39-41,44-$ 47), $P$. andersoni mcilhennyi $(51,55)$.
39. Río Curaray, Boca ( $=$ mouth), $2^{\circ} 22^{\prime} \mathrm{S}$, $74^{\circ} 05^{\prime} \mathrm{W}$.

Olalla Brothers, 1925.

40. Lago Mirañes, Río Napo (see Río Mazán).

40. Río Mazán (mouth, $3^{\circ} 28^{\prime} \mathrm{S}, 73^{\circ} 02^{\prime} \mathrm{W}$.

L. Söderström, September 1930.

41. Santa Luisa, Río Nanay, $3^{\circ} 35^{\prime} \mathrm{S}, 74^{\circ} 30^{\prime} \mathrm{W}$.

C. Kalinowski, October 1956.

42. Itaya (see Iquitos).

H. Bassler, 1927.

42. Iquitos, Río Marañon, $3^{\circ} 46^{\prime} \mathrm{S}, 73^{\circ} 15^{\prime} \mathrm{W}$, $106 \mathrm{~m}$.

H. Bassler, March 1928; C. Kalinowski, October 1956; R. W. Hendee, January 1928; C. Arevalo (H. Bassler collection), March 1930.

43. Apayacu, $3^{\circ} 19^{\prime} \mathrm{S}, 72^{\circ} 07^{\prime} \mathrm{W}$.

Olalla Brothers, January 1927.

44. Orosa, $3^{\circ} 26^{\prime} \mathrm{S}, 72^{\circ} 08^{\prime} \mathrm{W}$.

Ollalla Brothers, October 1926.

45. Nauta, $4^{\circ} 30^{\prime} \mathrm{S}, 73^{\circ} 25^{\prime} \mathrm{W}$, ca. $130 \mathrm{~m}$.

46. Santa Elena, Río Samiria, $4^{\circ} 42^{\prime} \mathrm{S}, 74^{\circ} 12^{\prime} \mathrm{W}$, ca. $130 \mathrm{~m}$.

C. Kalinowski, November 1956.

47. Yurimaguas, Río Huallaza, $5^{\circ} 54^{\prime} \mathrm{S}, 76^{\circ} 05^{\prime} \mathrm{W}$, $180 \mathrm{~m}$.

47. Yana Yacu, below Yurimaguas, $5^{\circ} 52^{\prime} \mathrm{S}$, $76^{\circ} 15^{\prime} \mathrm{W}$.

M. P. Anderson, September 1912, at $180 \mathrm{~m}$.

48. Sarayacu, Río Ucayali, $6^{\circ} 44^{\prime} \mathrm{S}, 75^{\circ} 06^{\prime} \mathrm{W}$.

A. M. Olalla, March, April 1927.

49. San Jerónimo, $7^{\circ} 45^{\prime} \mathrm{S}, 74^{\circ} 50^{\prime} \mathrm{W}, 300 \mathrm{~m}$.

R. W. Hendee, December 1927.

50. Yarinacocha, $8^{\circ} 15^{\prime} \mathrm{S}, 74^{\circ} 43^{\prime} \mathrm{W}, 160 \mathrm{~m}$.

C. C. Sanborn, March 1946; A. L. Gardner, August 1968, March 1971.

51. Pucallpa, $59 \mathrm{~km} \mathrm{~W}, 59 \mathrm{~km} \mathrm{NE}, 59 \mathrm{~km} \mathrm{SW}$ (see Pucallpa, Río Ucayali).

H. Hinse, October, November 1971, October 1972; H. Hinse, 59 km NE, September 1972; 59 km SW, October 1972.

51. Pucallpa, Río Ucayali, $8^{\circ} 23^{\prime} \mathrm{S}, 74^{\circ} 32^{\prime} \mathrm{W}, 180$ $\mathrm{m}$.

52. Cumeria (= Cumaría, across from Shahuía, q.v.).

52. Shahuía, Río Ucayali opposite Cumaría, $9^{\circ} 52^{\prime} \mathrm{S}, 74^{\circ} 01^{\prime} \mathrm{W}$.

R. W. Hendee, July 1927, at $312 \mathrm{~m}$.

53. Chicosa, $10^{\circ} 21^{\prime} \mathrm{S}, 74^{\circ} 00^{\prime} \mathrm{W}$, ca. $150 \mathrm{~m}$.

R. W. Hendee, September 1927.

54. Lagarto Cocha, Río Ucayali, $10^{\circ} 41^{\prime} \mathrm{S}$, $73^{\circ} 48^{\prime} \mathrm{W}$.

Olalla Brothers, January 1928.

54. Río Urubamba, mouth, $10^{\circ} 42^{\prime} \mathrm{S}, 73^{\circ} 42^{\prime} \mathrm{W}$. 
Olalla Brothers, October, November 1927.

54. Santa Rosa, $10^{\circ} 43^{\prime} \mathrm{S}, 73^{\circ} 53^{\prime} \mathrm{W}$.

Olalla Brothers, December 1927.

55. Balta, Río Curanja, $10^{\circ} 08^{\prime} \mathrm{S}, 71^{\circ} 15^{\prime} \mathrm{W}, 300$ $\mathrm{m}$.

A. L. Gardner, June 1968, March 1971; R. Thomas, 1971; A. L. Gardner, July, August 1966, February 1977, July 1968;

J. P. O'Neil, February 1971.

\section{Amazonas-Philander andersoni andersoni}

56a. La Poza, Río Santiago, $4^{\circ} 01^{\prime} \mathrm{S}, 77^{\circ} 47^{\prime} \mathrm{W}$.

J. L. Patton, August 1979, at $180 \mathrm{~m}$.

56b. Huampami, Río Cenepa.

J. L. Patton, July, August 1977, July 1978.

San Martín-Philander opossum quica

56c. Yurac Yacu, $5^{\circ} 52^{\prime} \mathrm{S}, 77^{\circ} 14^{\prime} \mathrm{W}$.

R. W. Hendee, July 1926, at $780 \mathrm{~m}$.

57. Moyobamba, Río Mayo, $6^{\circ} 03^{\prime} \mathrm{S}, 76^{\circ} 58^{\prime} \mathrm{W}$.

W. H. Osgood and M. P. Anderson, August 1912;

L. Rutter, February 1924, June 1926, at $845 \mathrm{~m}$.

58. Rioja, $6^{\circ} 10^{\prime} \mathrm{S}, 77^{\circ} 10^{\prime} \mathrm{W}$, ca. $800 \mathrm{~m}$.

R. W. Hendee, September 1926.

Huánuco_Philander opossum quica

59. Tingo María, $9^{\circ} 08^{\prime} \mathrm{S}, 75^{\circ} 57^{\prime} \mathrm{W}$.

R. W. Hendee, January 1927, at $625 \mathrm{~m}$.

\section{Pasco-Philander andersoni andersoni}

60. Oxapampa, $10^{\circ} 34^{\prime} \mathrm{S}, 75^{\circ} 24^{\prime} \mathrm{W}$.

C. R. Pérez, August 1964, at 280 m.

Junín-Philander andersoni andersoni

61. Chanchamayo, $11^{\circ} 03^{\prime} \mathrm{S}, 75^{\circ} 19^{\prime} \mathrm{W}$. P. O. Simons, August 1900; J. M. Schunke, August 1948; M. Kalinowski before 1916.

61. Utcuyacu, $11^{\circ} 12^{\prime} \mathrm{S}, 75^{\circ} 28^{\prime} \mathrm{W}$.

P. O. Simons, August 1900, at $1600 \mathrm{~m}$.
Ayacucho-Philander andersoni andersoni

62. San José, Río Santa Rosa, $12^{\circ} 44^{\prime} \mathrm{S}, 73^{\circ} 46^{\prime} \mathrm{W}$. A. L. Gardner, April 1971, at $1000 \mathrm{~m}$.

62. Huanhuachayo, $12^{\circ} 44^{\prime} \mathrm{S}, 73^{\circ} 47^{\prime} \mathrm{W}$.

A. L. Gardner, May 1971, at $1660 \mathrm{~m}$.

\section{Cuzco-Philander opossum quica}

63. Hacienda Cadena, Marcapata, $13^{\circ} 24^{\prime} \mathrm{S}$, $70^{\circ} 43^{\prime} \mathrm{W}, 890 \mathrm{~m}$.

C. Kalinowski, October 1949, August 1950.

63. San Jerónimo, $13^{\circ} 34^{\prime} \mathrm{S}, 71^{\circ} 54^{\prime} \mathrm{W}$.

64. Quincemil, $13^{\circ} 16^{\prime} \mathrm{S}, 70^{\circ} 38^{\prime} \mathrm{W}$.

C. Kalinowski, June 1953, at $680 \mathrm{~m}$.

Madre de Dios-Philander opossum quica

65. Colorado, Río (mouth) (see Boca Colorado).

65. Boca Colorado, $12^{\circ} 30^{\prime} \mathrm{S}, 70^{\circ} 10^{\prime} \mathrm{W}$.

C. Kalinowski, October 1954, at $279 \mathrm{~m}$.

66. Puerto Maldonado, Río Tambo, $12^{\circ} 36^{\prime} \mathrm{S}$, $69^{\circ} 11^{\prime} \mathrm{W}, 256 \mathrm{~m}$.

M. L. Kuns, April 1965.

Bolivia-Philander opossum quica

La Paz

67. Culumani (see Chulumani).

67. Chulumani, $16^{\circ} 24^{\prime} \mathrm{S}, 67^{\circ} 31^{\prime} \mathrm{W}, 2000 \mathrm{~m}$.

P. O. Simons, February 1901, at 2200 m.

El Beni

68. Guayaramarín, Río Mamoré, $10^{\circ} 51^{\prime} \mathrm{S}$, $65^{\circ} 23^{\prime} \mathrm{W}$

A. Ximénez, June 1964; K. F. Koopman, 1964.

69. Merredor, Río Mamoré, $11^{\circ} 41^{\prime} \mathrm{S}, 65^{\circ} 05^{\prime} \mathrm{W}$.

D. Edwards, July 1965; A. Ximénez, May 1965.

70. Río Mamoré, $12^{\circ} 26^{\prime} \mathrm{S}$, ca. $65^{\circ} \mathrm{W}$.

D. E. Añez, October 1965.

71. Itonama, $12^{\circ} 28^{\prime} \mathrm{S}, 64^{\circ} 24^{\prime} \mathrm{W}$.

M. L. Kuns, April 1970.

72. Puerto Siles, Río Mamoré, $12^{\circ} 49^{\prime} \mathrm{S}$, $65^{\circ} 00^{\prime} \mathrm{W}$.

S. Anderson, October 1965. 
72. Santa Rosa, $13^{\circ} 01^{\prime} \mathrm{S}, 65^{\circ} 11^{\prime} \mathrm{W}$. M. L. Kuns, June 1964.

73. Barranquita (see San Joaquín). M. L. Kuns, August 1963.

73. Arruda, $4 \mathrm{~km}$ NE San Joaquín (q.v.). M. L. Kuns, December 1963.

73. Centinela, $1.5 \mathrm{~km}$ E San Joaquín (q.v.).

M. L. Kuns, June 1964.

73. Camino Vilches, 1 mi E San Joaquín (q.v.).

M. L. Kuns, February 1966.

73. Santo Dios, 2 km NE San Joaquín (q.v.).

M. L. Kuns, June 1963.

73. San Joaquín, Río Machupo, $13^{\circ} 04^{\prime} S$, $64^{\circ} 49^{\prime} \mathrm{W}$.

M. L. Kuns, April, June, July 1963; February, May 1964.

74. Exaltación, Río Mamoré, $13^{\circ} 16^{\prime} \mathrm{S}, 65^{\circ} 15^{\prime} \mathrm{W}$.

M. L. Kuns, May 1964; S. Anderson, October 1965, at $8 \mathrm{~km} \mathrm{~N}$.

74. Palacios, $13^{\circ} 34^{\prime} \mathrm{S}, 65^{\circ} 19^{\prime} \mathrm{W}$.

D. E. Añez, May 1965.

74. Puerto Caballo, $13^{\circ} 34^{\prime} \mathrm{S}, 65^{\circ} 2 l^{\prime} \mathrm{W}$.

S. Anderson, September 1965.

75. San Ramón, Río Machupo, $13^{\circ} 18^{\prime} \mathrm{S}$, $64^{\circ} 37^{\prime} \mathrm{W}$.

M. L. Kuns, January 1964.

75. Monte Chocolatal (see San Ramón).

M. L. Kuns, January 1964.

76. Magdalena, $13^{\circ} 20^{\prime} \mathrm{S}, 64^{\circ} 08^{\prime} \mathrm{W}$.

M. L. Kuns, July 1963, July 1964.

77. San Pablo, $13^{\circ} 52^{\prime} \mathrm{S}, 65^{\circ} 36^{\prime} \mathrm{W}$.

M. L. Kuns, September 1963.

78. El Carmen, Río Blanco, $13^{\circ} 57^{\prime} \mathrm{S}, 63^{\circ} 43^{\prime} \mathrm{W}$.

M. L. Kuns, June 1964.

79. Río Ibaré (mouth), $14^{\circ} 37^{\prime} \mathrm{S}, 64^{\circ} 57^{\prime} \mathrm{W}$.

D. E. Añez, August 1965.

K. F. Koopman, August 1965.

79. Vaca Diez, $14^{\circ} 47^{\prime} \mathrm{S}, 64^{\circ} 51^{\prime} \mathrm{W}$.

M. L. Kuns, May 1969.

80. San Ignacio de Móxos, $14^{\circ} 53^{\prime} \mathrm{S}, 65^{\circ} 36^{\prime} \mathrm{W}$.

M. L. Kuns, June 1965.

81. Camiaco, $15^{\circ} 24^{\prime} \mathrm{S}, 64^{\circ} 46^{\prime} \mathrm{W}$.

A. Ximénez, August 1965.

Cochabamba

82. Río Ichilo, $16^{\circ} 50^{\prime} \mathrm{S}, 64^{\circ} 45^{\prime} \mathrm{W}$.

D. E. Añez, July 1965.

Santa Cruz

83. $2 \mathrm{~km} \mathrm{~S}$ mouth, Río Chaparé, Río Mamoré, $15^{\circ} 58^{\prime} \mathrm{S}, 64^{\circ} 42^{\prime} \mathrm{W}$.

D. E. Añez, July 1965.
84. Ascención de Guarayos, $14^{\circ} 57^{\prime} \mathrm{S}, 61^{\circ} 24^{\prime} \mathrm{W}$. M. L. Kuns, June 1964.

85. Mercedes, Río Guaporé, $15^{\circ} 36^{\prime} \mathrm{S}, 60^{\circ} 22^{\prime} \mathrm{W}$, $6 \mathrm{~km} \mathrm{~S}$ and opposite Buena Hora, Brasil.

A. Ximénez, May 1965.

86. Buenavista, $17^{\circ} 27^{\prime} \mathrm{S}, 63^{\circ} 40^{\prime} \mathrm{W}$.

T. Bridges, probably 1846; F. Steinbach, January 1915; J. Steinbach, June 1921, May 1925, July 1926, June, July 1927, July 1928.

87. Warnes, $17^{\circ} 31^{\prime} \mathrm{S}, 63^{\circ} 10^{\prime} \mathrm{W}$.

M. L. Kuns, August 1965.

87. Santa Rosita, Warnes, $17^{\circ} 30^{\prime} \mathrm{S}, 63^{\circ} 10^{\prime} \mathrm{W}$.

M. L. Kuns, July 1965.

88. Tocomechi, $17^{\circ} 35^{\prime} \mathrm{S}, 62^{\circ} 55^{\prime} \mathrm{W}$.

M. L. Kuns, August 1965.

88. Hamacas, $17^{\circ} 44^{\prime} \mathrm{S}, 63^{\circ} 1 \mathrm{I}^{\prime} \mathrm{W}$.

O. Silva (Rockefeller Institution), July 1938.

88. El Palmar, $17^{\circ} 48^{\prime} \mathrm{S}, 63^{\circ} 10^{\prime} \mathrm{W}$.

W. Kerr, August 1966, at $500 \mathrm{~m}$; D. R. Hadden, August 1966.

88. Ayacucho, lbáñez, $17^{\circ} 51^{\prime} \mathrm{S}, 63^{\circ} 20^{\prime} \mathrm{W}$.

J. Riddell, August 1966.

88. 18 km SW Santa Cruz (q.v.).

F. Becerra, August 1966.

88. Santa Cruz (see Santa Cruz de la Sierra).

88. Santa Cruz de la Sierra, $17^{\circ} 48^{\prime} \mathrm{S}, 63^{\circ} 10^{\prime} \mathrm{W}$.

F. Becerra, September 1966.

89. San Ramón, $17^{\circ} 33^{\prime} \mathrm{S}, 61^{\circ} 03^{\prime} \mathrm{S}$.

Paraguay_Philander opossum quica

San Pedro

90. Tacuati, $23^{\circ} 27^{\prime} \mathrm{S}, 56^{\circ} 35^{\prime} \mathrm{W}$.

C. Wharton, May 1950.

Central

91. Asunción, $25^{\circ} 16^{\prime} \mathrm{S}, 57^{\circ} 40^{\prime} \mathrm{W}$.

92. Lapango, $25^{\circ} 21^{\prime} \mathrm{S}, 57^{\circ} 42^{\prime} \mathrm{W}$.

H. Krieg, August 1925.

Paraguarí

93. Sapucay (= Sapucaí), $25^{\circ} 40^{\prime} \mathrm{S}, 56^{\circ} 55^{\prime} \mathrm{W}$.

W. Foster, September 1902.

Argentina-Philander opossum

Chaco

94. No precise locality.

C. Friend, before 1880 . 
94. Río de Oro (mouth), $27^{\circ} 04^{\prime} \mathrm{S}, 58^{\circ} 34^{\prime} \mathrm{W}$.

I. Apostal, October 1962.

Misiones

95. Río Uruguar-í, $30 \mathrm{~km}$ from Puerto Bemberg (Libertad). $26^{\circ} 30^{\prime} \mathrm{S}, 54^{\circ} \mathrm{W}$.

J. A. Crespo, September, October, November 1949.

96. Fracrán, San Pedro, $26^{\circ} 46^{\prime} \mathrm{S}, 54^{\circ} 16^{\prime} \mathrm{W}$.

J. A. Crespo, February 1952.

Venezuela-Philander opossum subspecies, $P$. andersoni andersoni

Zulia-Philander opossum subspecies

97a. Alguacil, Caja Seca, $09^{\circ} 8^{\prime} \mathrm{N}, 71^{\circ} 04^{\prime} \mathrm{W}$.

97a. Churulí, Caja Seca, $09^{\circ} 09^{\prime} \mathrm{N}, 71^{\circ} 04^{\prime} \mathrm{W}$.

97b. Puerto Catatumbo, $09^{\circ} 07^{\prime} \mathrm{N}, 72^{\circ} 35^{\prime} \mathrm{W}$, $50 \mathrm{~m}$.

97b. Boca del Río de Oro, $9^{\circ} 06^{\prime} \mathrm{N}, 72^{\circ} 45^{\prime} \mathrm{W}$.

97b. Encontrados, $9^{\circ} 03^{\prime} \mathrm{S}, 72^{\circ} 14^{\prime} \mathrm{W}$.

N. E. Peterson, March 1968.

97b. Encontrados, $60 \mathrm{~km}$ WNW, $90^{\circ} 03^{\prime} \mathrm{N}$, $72^{\circ} 14^{\prime} \mathrm{W}, 73 \mathrm{~m}$.

Trujillo-Philander opossum subspecies

98a. Motatán, $9^{\circ} 24^{\prime} \mathrm{N}, 70^{\circ} 36^{\prime} \mathrm{W}, 1 \mathrm{~km} \mathrm{E}$ at 330 $\mathrm{m}, 5 \mathrm{~km} \mathrm{NNE}$ at $290 \mathrm{~m}$.

J. A. W. Kirsch, June 1969.

98a. Motatán (Río), $9^{\circ} 28^{\prime} \mathrm{N}, 70^{\circ} 34^{\prime} \mathrm{W}$ at $290 \mathrm{~m}$, $9.8 \mathrm{~km} \mathrm{NNE}$ at $290 \mathrm{~m}$.

J. A. W. Kirsch, June 1969.

98a. Agua Viva, $9^{\circ} 34^{\prime} \mathrm{N}, 70^{\circ} 36^{\prime} \mathrm{W}$.

N. E. Peterson, September 1965, at $164 \mathrm{~m}$.

98a. El Dividive, $9^{\circ} 29^{\prime} \mathrm{N}, 70^{\circ} 44^{\prime} \mathrm{W}$.

N. E. Peterson, October 1965 , at $90 \mathrm{~m}$.

Monagas-Philander [?] opossum subspecies

98b. Papelón (Cerro), $10^{\circ} 01^{\prime} \mathrm{N}, 63^{\circ} 54^{\prime} \mathrm{W}$.

Delta Amacuro (Territorio Federal)-Philander opossum subspecies

98c. Los Guires, $09^{\circ} 15^{\prime} \mathrm{N}, 61^{\circ} 54^{\prime} \mathrm{W}$.

98c. Tobesobe, Guayo, $09^{\circ} 00^{\prime} \mathrm{N}, 61^{\circ} 25^{\prime} \mathrm{W}$.

98d. Güiniquina, $90^{\circ} 10^{\prime} \mathrm{N}, 61^{\circ} 03^{\prime} \mathrm{W}, 0 \mathrm{~m}$.
Mérida-Philander opossum subspecies

99. “Merida," $8^{\circ} 36^{\prime} \mathrm{N}, 71^{\circ} 08^{\prime} \mathrm{W}$.

Barinas-Philander opossum subspecies

100a. Ticoporo, Reserva Forestal, $07^{\circ} 48^{\prime} \mathrm{N}$, $69^{\circ} 55^{\prime} \mathrm{W}$.

100b. Guaquitas, $07^{\circ} 28^{\prime} \mathrm{N}, 71^{\circ} 39^{\prime} \mathrm{W}$.

100b. Las Bonitas, Caño Amaru, $07^{\circ} 23^{\prime} \mathrm{N}$, $70^{\circ} 44^{\prime} \mathrm{W}$.

100c. La Lengueta, $08^{\circ} 30^{\prime} \mathrm{N}, 70^{\circ} 23^{\prime} \mathrm{W}$.

Apure-Philander opossum subspecies

101a. Nulita, $07^{\circ} 19^{\prime} \mathrm{N}, 71^{\circ} 55^{\prime} \mathrm{W}$.

A. Tuttle, January 1968 , at $24 \mathrm{~m}$.

101a. La Blanquita, $7^{\circ} 12^{\prime} \mathrm{N}, 71^{\circ} 45^{\prime} \mathrm{W}$.

A. Tuttle, January 1968, at $24 \mathrm{~m}$.

Táchira-Philander opossum subspecies

101b. La Ponchera, $07^{\circ} 26^{\prime} \mathrm{N}, 71^{\circ} 52^{\prime} \mathrm{S}$.

Bolívar-Philander opossum opossum

102a. El Palmar, Río Grande, $08^{\circ} 01^{\prime} \mathrm{N}, 61^{\circ} 55^{\prime} \mathrm{W}$.

102b. Maripa, Río Caura, $7^{\circ} 26^{\prime} \mathrm{N}, 65^{\circ} 09^{\prime} \mathrm{W}$.

S. M. Klages, 1901.

103. Hato San José, 146 km S, 7 km NE Ciudad Bolívar, $6^{\circ} 44^{\prime} \mathrm{N}, 63^{\circ} 27^{\prime} \mathrm{W}$.

N. Peterson, D. Peacock, R. Peacock, D. Furman, March 1967, at $302 \mathrm{~m}$.

104a. San Martín de Turumbán, Río Cuyuni, $06^{\circ} 59^{\prime} \mathrm{N}, 61^{\circ} 02^{\prime} \mathrm{W}$.

104b. Río Yuruán, $06^{\circ} 48^{\prime} \mathrm{N}, 61^{\circ} 50^{\prime} \mathrm{W}$.

M. A. Carriker, Jr., March 1910.

104c. Chalimana (Raudal), Río Paramichí, Río Paragua, $04^{\circ} 10^{\prime} \mathrm{N}, 62^{\circ} 59^{\prime} \mathrm{W}$.

Amazonas (Territorio Federal)-Philander andersoni andersoni

105. Majagua (Caño), Rio Ventuari, $05^{\circ} 20^{\prime} \mathrm{N}$, $65^{\circ} 40^{\prime} \mathrm{W}$.

105. San Juan, Río Manapiare, $05^{\circ} 19^{\prime} \mathrm{N}$, $66^{\circ} 03^{\prime} \mathrm{W}$.

M. D. Tuttle, F. L. Harder, July 1967, at $155 \mathrm{~m}$.

106. Belén, Río Cunucunumá, $03^{\circ} 43^{\prime} \mathrm{N}, 65^{\circ} 42^{\prime} \mathrm{W}$. 
M. D. Tuttle, F. L. Harder, January 1967, at $150 \mathrm{~m}$.

107. Acanaña, Río Cunucunumá, $03^{\circ} 39^{\prime} \mathrm{N}$, $65^{\circ} 66^{\prime} \mathrm{W}$.

M. D. Tuttle, F. L. Harder, June 1967, at $145 \mathrm{~m}$

108. Playa del Río Base, Mt. Duida, $03^{\circ} 25^{\prime} \mathrm{N}$, $65^{\circ} 40^{\prime} \mathrm{W}$

Olalla Brothers, November 1928.

109a. Esmeralda, $03^{\circ} 11^{\prime} \mathrm{N}, 65^{\circ} 33^{\prime} \mathrm{W}$.

M. D. Tuttle, F. L. Harder, March 1967, at $135 \mathrm{~m}$.

109b. Cucurito, Cerro, $03^{\circ} 38^{\prime} \mathrm{N}, 66^{\circ} 25^{\prime} \mathrm{W}$.

110a. Tamatama, Río Orinoco, $3^{\circ} 08^{\prime} \mathrm{N}, 65^{\circ} 52^{\prime} \mathrm{W}$.

M. D. Tuttle, F. L. Harder, April, May, June 1967, at 135 m; D. S. Bremington, June 1967. at $135 \mathrm{~m}$.

110b. Parima (Sierra), $02^{\circ} 40^{\prime} \mathrm{N}, 64^{\circ} 30^{\prime} \mathrm{W}$.

110c. Mavaca, $02^{\circ} 31^{\prime} \mathrm{N}, 65^{\circ} 10^{\prime} \mathrm{W}$.

111. Capibara, Brazo Casiquiare, $2^{\circ} 34^{\prime} \mathrm{N}$, $66^{\circ} 18^{\prime} \mathrm{W}$.

M. D. Tuttle, F. L. Harder, June 1967, at $130 \mathrm{~m}$.

112a. Merey, opposite, Brazo Casiquiare, $2^{\circ} 17^{\prime} \mathrm{N}$, $67^{\circ} 11^{\prime} \mathrm{W}$.

Olalla Brothers, October 1929.

112b. La Neblina (Campamento), Cerro La Neblina, $00^{\circ} 52^{\prime} \mathrm{N}, 66^{\circ} 14^{\prime} \mathrm{W}$.

Guyana-Philander opossum opossum

Demerara-Mahaica

113. Supinaam River (= Supenam River), $6^{\circ} 58^{\prime} \mathrm{N}, 58^{\circ} 31^{\prime} \mathrm{W}$.

Crozier.

114. Georgetown, Demerara River, $6^{\circ} 48^{\prime} \mathrm{N}$, $58^{\circ} 10^{\prime} \mathrm{W}$.

J. Rodway, June 1929.

115. Hyde Park, Demerara River, $6^{\circ} 30^{\prime} \mathrm{N}$, $58^{\circ} 16^{\prime} \mathrm{W}$.

S. B. Warren, September 1906.

Essequibo Islands-West Demerara

116. Buck Hall, Essequibo River, $6^{\circ} 56^{\prime} \mathrm{N}$, $58^{\circ} 33^{\prime} \mathrm{W}$.

S. B. Warren, March 1906.

Upper Takutu-Upper Essequibo

117. Rupununi River, $4^{\circ} 03^{\prime} \mathrm{N}, 58^{\circ} 34^{\prime} \mathrm{W}$.

J. J. Quelch, September 1900, at $200 \mathrm{ft}$.
118. Kanuku Mountains, $3^{\circ} \mathrm{N}, 59^{\circ} 45^{\prime} \mathrm{W}$.

E. V. McConnell, J. J. Quelch, November 1900 , at $240 \mathrm{ft}$.

Suriname-Philander opossum opossum

Nickerie

119. Avanavero Falls, Kabulebo River, $4^{\circ} 49^{\prime} \mathrm{N}$, $57^{\circ} 24^{\prime} \mathrm{W}$

120. Nickerie River, upper, ca. $5^{\circ} 59^{\prime} \mathrm{N}, 56^{\circ} 30^{\prime} \mathrm{W}$.

121. Makerie, West River, Wilhelmina Mountains, $3^{\circ} 26^{\prime} \mathrm{N}, 56^{\circ} 45^{\prime} \mathrm{W}$.

H. A. Beatty, December 1961, January 1962.

122. Kaiserberg Airstrip, $3^{\circ} 10^{\prime} \mathrm{N}, 56^{\circ} 15^{\prime} \mathrm{W}$.

H. A. Beatty, February 1961, at $275 \mathrm{~m}$.

Brokopondo

123. Loksie Hattie, $5^{\circ} 09^{\prime} \mathrm{N}, 55^{\circ} 28^{\prime} \mathrm{W}$.

P. Hershkovitz, December 1961.

Saramacca

124. La Poule, $5^{\circ} 47^{\prime} \mathrm{N}, 55^{\circ} 25^{\prime} \mathrm{W}$.

P. Hershkovitz, January, February 1962.

Suriname

125. Paramaribo, $5^{\circ} 50^{\prime} \mathrm{N}, 55^{\circ} 1 \mathrm{I}^{\prime} \mathrm{W}$

I. T. Sanderson, February 1938.

125. Clevia, Paramaribo (q.v.).

P. Hershkovitz, February 1962.

125. "Culturutuin," Agricultural Experimental Station in Paramaribo (q.v.).

125. Para River, ca. 10 km SE Paramaribo (q.v.).

125. Rijweg, ca. $9 \mathrm{~km}$ W Paramaribo (q.v.).

126. Lelydorp, $5^{\circ} 42^{\prime} \mathrm{N}, 55^{\circ} 16^{\prime} \mathrm{W}$.

126. Lelydorpplan (see Lelydorp).

P. Hershkovitz, January, February 1962.

Commewijne

127. Commewijne River, $5^{\circ} 54^{\prime} \mathrm{N}, 55^{\circ} 05^{\prime} \mathrm{W}$.

Marowijne

128. Albina, $5^{\circ} 30^{\prime} \mathrm{N}, 54^{\circ} 03^{\prime} \mathrm{W}$. 
Guyane Française-Philander opossum opossum

Cayenne

129. Cayenne, $4^{\circ} 56^{\prime} \mathrm{N}, 52^{\circ} 19^{\prime} \mathrm{W}$.

G. K. Cherrie, B. T. Gault, November 1902; S. Klages, January, February 1917; M. Atramentowicz, September 1978October 1982; P. Charles-Dominique, 19781982.

\section{Ouanary}

130. Ouanary River, $4^{\circ} 14^{\prime} \mathrm{N}, 51^{\circ} 39^{\prime} \mathrm{W}$.

Brasil-Philander opossum opossum, P. opossum frenata, $P$. opossum quica

Amapá-Philander opossum opossum

131. Serra do Navio, $0^{\circ} 59^{\prime} \mathrm{N}, 52^{\circ} 03^{\prime} \mathrm{W}$.

T. P. Woodall, June 1966; Instituto Evandro Chagas, 1967, 1968, 1969.

131. Teresinha (= Terezinha), Río Amapari, $0^{\circ} 58^{\prime} \mathrm{N}, 52^{\circ} 02^{\prime} \mathrm{W}$.

132. Macapá, Rio Amapari, $0^{\circ} 02^{\prime} \mathrm{N}, 51^{\circ} 03^{\prime} \mathrm{W}$.

M. Moreira, October, November 1952.

133. Mazagão, Rio Maracá, $0^{\circ} 06^{\prime} \mathrm{S}, 51^{\circ} 18^{\prime} \mathrm{W}$.

M. Moreira, December 1958.

133. Rio Branco, tributary Rio Maracá, $0^{\circ} 07^{\prime} \mathrm{S}$, $51^{\circ} 17^{\prime} \mathrm{W}$.

\section{Roraima-Philander opossum opossum}

134. Bõa Vista, Río Branco, $2^{\circ} 49^{\prime} \mathrm{N}, 60^{\circ} 40^{\prime} \mathrm{W}$.

C. T. Carvalho, M. Sobeiro do Amaral, M. Melo, March 1959.

134. Pocão, Bõa Vista (see Bõa Vista).

135. Caracaraí, Rio Mucajai, $1^{\circ} 50^{\prime} \mathrm{N}, 61^{\circ} 08^{\prime} \mathrm{W}$.

C. T. Carvalho, M. Sobeiro do Amaral, M. Melo, March 1959.

135. Pocão, Caracaraí (see Caracaraí).

Amazonas-Philander opossum opossum

136. Rio Xiriviny, $0^{\circ} 59^{\prime} \mathrm{S}, 61^{\circ} 53^{\prime} \mathrm{W}$.

K. B. Parker, October 1928, at $80 \mathrm{~m}$.

137. Santo Isidoro, Tefé, $3^{\circ} 27^{\prime} \mathrm{S}, 64^{\circ} 47^{\prime} \mathrm{W}$.

Olalla Brothers, August 1928.

138. Ipixuna (Lago do), $3^{\circ} 52^{\prime} \mathrm{S}, 63^{\circ} 52^{\prime} \mathrm{W}$.
139. Serra de Parintins, $2^{\circ} 35^{\prime} \mathrm{S}, 56^{\circ} 25^{\prime} \mathrm{W}$.

Olalla Brothers, November 1930.

139. Parintins, $2^{\circ} 36^{\prime} \mathrm{S}, 56^{\circ} 44^{\prime} \mathrm{W}$.

Olalla Brothers, November 1930.

140. Santa Clara, Villa Bella Imperatriz, $2^{\circ} 50^{\prime} \mathrm{S}$, $56^{\circ} 55^{\prime} \mathrm{W}$.

Olalla Brothers, August 1930.

141. Lago do Baptista, $3^{\circ} 18^{\prime} \mathrm{S}, 58^{\circ} 15^{\prime} \mathrm{W}$.

A. M. Olalla, June 1936.

142. Río Madeira, mouth, $3^{\circ} 22^{\prime} \mathrm{S}, 58^{\circ} 45^{\prime} \mathrm{W}$.

Olalla Brothers, February 1930.

143. Rosarhino, $3^{\circ} 43^{\prime} \mathrm{S}, 59^{\circ} 08^{\prime} \mathrm{W}$. Olalla Brothers, June, July 1930.

144. Auará Igarapé, $4^{\circ} 22^{\prime} \mathrm{S}, 59^{\circ} 43^{\prime} \mathrm{W}$.

Olalla Brothers, March 1930.

145a. Santo Antônio de Uayara, Rio Eirú, $6^{\circ} 43^{\prime}$ S, $69^{\circ} 52^{\prime} \mathrm{W}$.

Olalla Brothers, April 1930.

145b. Río Urucu, $4^{\circ} 51^{\prime} \mathrm{S}, 65^{\circ} 16^{\prime} \mathrm{W}$.

M. N. F. da Silva.

Acre-Philander opossum quica

146. Seringal Oriente, $8^{\circ} 48^{\prime} \mathrm{S}, 72^{\circ} 46^{\prime} \mathrm{W}$.

M. Moreira, August 1934; M. Moreira, F.

Novaes, August 1956.

Pará-Philander opossum opossum

147. Fordlandia, Rio Tapajóz, $3^{\circ} 40^{\prime} \mathrm{S}, 55^{\circ} 30^{\prime} \mathrm{W}$.

R. M. Gilmore, February 1938.

148. $\mathrm{Km} \mathrm{19}$, Itaituba-Jacareacanga, $4^{\circ} 17^{\prime} \mathrm{S}$, $56^{\circ} 05^{\prime} \mathrm{W}$.

F. Ramos, Instituto Oswaldo Cruz, August 1972.

148. Km 216, Estrada de Santarem-Cuiabá (BR 165) (see Itaituba).

Instituto Evandro Chagas, May, June 1973.

149. Canudos, $7^{\circ} 16^{\prime} \mathrm{S}, 58^{\circ} 07^{\prime} \mathrm{W}$.

F. Lima, November 1920.

150. Taperebá, Igarapé, Chaves, $0^{\circ} 10^{\prime} \mathrm{S}, 49^{\circ} 55^{\prime} \mathrm{W}$. C. Carvalho, June, July 1958.

151. Altamira, Rio Xingú, $3^{\circ} 12^{\prime} \mathrm{S}, 52^{\circ} 12^{\prime} \mathrm{W}$.

Instituto Oswaldo Cruz, August 1971.

152. Gradaús, $7^{\circ} 43^{\prime} \mathrm{S}, 51^{\circ} 11^{\prime} \mathrm{W}$.

C. T. Carvalho, N. Hidasi, M. Amaral, June, July, August 1957.

153. Cametá, Río Tocantins, $2^{\circ} 15^{\prime} \mathrm{S}, 49^{\circ} 29^{\prime} \mathrm{W}$.

A. M. Olalla, February 1934, March, April, May, November 1935, November 1936. 
153. Ilha do Taiuna, Río Tocantins, $2^{\circ} 15^{\prime} \mathrm{S}$, $49^{\circ} 29^{\prime} \mathrm{W}$, opposite Cametá.

A. M. Olalla, October, November 1931.

154. Mazagão, Rio Tocantins, $2^{\circ} 25^{\prime} \mathrm{S}, 49^{\circ} 10^{\prime} \mathrm{W}$.

F. Lima, November 1912.

155. Mocajuba, Río Tocantins, $2^{\circ} 35^{\prime} \mathrm{S}, 49^{\circ} 30^{\prime} \mathrm{W}$.

A. M. Olalla, November 1931.

156. Baião, Río Tocantins, $2^{\circ} 41^{\prime} \mathrm{S}, 49^{\circ} 41^{\prime} \mathrm{W}$.

A. M. Olalla, December 1931.

157. Arumateua, Río Tocantins, $3^{\circ} 54^{\prime} \mathrm{S}, 49^{\circ} 41^{\prime} \mathrm{W}$.

F. Lima, October 1912.

158. Marabá, Río Tocantíns, $5^{\circ} 20^{\prime} \mathrm{S}, 4^{\circ} 10^{\prime} \mathrm{W}$.

N. Peterson, September 1975.

159. Pará (see Belém).

159. Km 90, Pará, Rodovia

E. Snethlage, April 1909.

159. Belém do Pará (see Belém).

159. Belém, $1^{\circ} 27^{\prime} \mathrm{S}, 48^{\circ} 29^{\prime} \mathrm{W}$.

M. A. Miles, 1975-1980.

159. Ipeau-Apez, Belém (see Belém).

E. Snethlage.

159. Marco, suburb of Belém (q.v.).

159. Murutucu (= Instituto Agronomico do Norte), Belém (q.v.).

F. Luna, August 1922, March 1925.

159. Utinga, suburb of Belém (q.v.).

Instituto Oswaldo Cruz, June 1963; R. H. Pine, June 1968.

159. Ananindéua, E. F. Bragança, $1^{\circ} 22^{\prime} \mathrm{S}$, $48^{\circ} 23^{\prime} \mathrm{W}$.

F. Lima, May 1920.

159. Sapucajuba, suburb of Belém (q.v.).

160. Ipitinga, Rio Acará, $1^{\circ} 57^{\prime} \mathrm{S}, 48^{\circ} 11^{\prime} \mathrm{W}$.

161. Flor do Prado, near Quatipuru, $0^{\circ} 52^{\prime} \mathrm{S}$, $46^{\circ} 59^{\prime} \mathrm{W}$.

E. Snethlage, October 1916.

162. Santa María, Bragança, $1^{\circ} 03^{\prime} \mathrm{S}, 46^{\circ} 46^{\prime} \mathrm{W}$. April 1968.

163. Peixe-Boi, R. R. Bragança, $1^{\circ} 12^{\prime} \mathrm{S}, 47^{\circ} 18^{\prime} \mathrm{W}$.

163. Igarapé Açu, $1^{\circ} 32^{\prime} \mathrm{S}, 47^{\circ} 03^{\prime} \mathrm{W}$.

163. Colônia do Prata, Igarapé Açu (q.v.).

164. Capim, $1^{\circ} 30^{\prime} \mathrm{S}, 48^{\circ} 20^{\prime} \mathrm{W}$.

Instituto Oswaldo Cruz, 1960.

165. São Miguel do Guamá (see Guamá).

165. Guamá, $1^{\circ} 37^{\prime} \mathrm{S}, 47^{\circ} 27^{\prime} \mathrm{W}$.

Departamento do Zoologia, São Paulo, October 1959.

165. Iriteria, São Miguel do Guamá (see Guamá).

M. Amaral, December 1959, January 1960.

\section{Rondônia-Philander opossum quica}

166. Porto Velho, $8^{\circ} 46^{\prime} \mathrm{S}, 63^{\circ} 54^{\prime} \mathrm{W}$.

T. Hibbs, April 1965.
Mato Grosso-Philander opossum quica

167. Mato Grosso, $15^{\circ} 00^{\prime} \mathrm{S}, 59^{\circ} 57^{\prime} \mathrm{W}$.

J. Natterer, September-November 1824.

168a. Cáceres, $16^{\circ} 04^{\prime} \mathrm{S}, 57^{\circ} 41^{\prime} \mathrm{W}$.

M. L. Kuns, September 1965.

168b. Poconé, $16^{\circ} 15^{\prime} \mathrm{S}, 56^{\circ} 37^{\prime} \mathrm{W}$.

168c. Barra do Garçes, $15^{\circ} 53^{\prime} \mathrm{S}, 52^{\circ} 15^{\prime} \mathrm{W}$,

168d. Balisa, $16^{\circ} 15^{\prime} \mathrm{S}, 52^{\circ} 26^{\prime} \mathrm{W}$.

169. Monte Alegre, Palmeiras, $16^{\circ} 03^{\prime} \mathrm{S}, 55^{\circ} 30^{\prime} \mathrm{W}$. A. M. Olalla, June 1944; A. Aggio, June 1944.

Mato Grosso do Sul-Philander opossum quica

170. Corumbá, Río Paraguay, $19^{\circ} 01^{\prime} \mathrm{S}, 57^{\circ} 39^{\prime} \mathrm{W}$. M. L. Kuns, September 1965.

170. Urucum, $19^{\circ} 13^{\prime} \mathrm{S}, 57^{\circ} 33^{\prime} \mathrm{W}$.

L. E. Miller, December 1913, at 125 m.

170. Santa Teresa (see Urucum).

M. L. Kuns, September 1965.

171 a. Miranda, $20^{\circ} 14^{\prime} \mathrm{S}, 56^{\circ} 22^{\prime} \mathrm{W}$.

A. M. Olalla, 1957; J. Lima, September 1937.

171b. Salobra, $21^{\circ} 14^{\prime} \mathrm{S}, 57^{\circ} 08^{\prime} \mathrm{W}$.

L. Travassos, May 1942.

Bahia-Philander opossum frenata

172. Bahia (= Salvador), $12^{\circ} 59^{\prime} \mathrm{S}, 38^{\circ} 31^{\prime} \mathrm{W}$.

Herr Kaehne, before 1815.

Goiás-Philander opossum quica

173. Aragarças, $15^{\circ} 55^{\prime} \mathrm{S}, 52^{\circ} 15^{\prime} \mathrm{W}$. M. Amaral, May 1958.

174a. Formosa, $15^{\circ} 32^{\prime} \mathrm{S}, 47^{\circ} 20^{\prime} \mathrm{W}$.

174b. Anapolis, $16^{\circ} 20^{\prime} \mathrm{S}, 48^{\circ} 58^{\prime} \mathrm{W}$.

R. M. Gilmore, 1936-1937.

175a. Trinidade, $16^{\circ} 40^{\prime} \mathrm{S}, 49^{\circ} 30^{\prime} \mathrm{W}$.

S. Hidasi, June 1962.

Distrito Federal-Philander opossum quica

175b. Parque Nacional, $15^{\circ} 35^{\prime} \mathrm{S}, 48^{\circ} 54^{\prime} \mathrm{W}$.

175c. Brasília, $15^{\circ} 47^{\prime} \mathrm{S}, 47^{\circ} 55^{\prime} \mathrm{W}$.

175c. Agua Limpa (Fazenda), $15^{\circ} 57^{\prime} \mathrm{S}, 47^{\circ} 54^{\prime} \mathrm{W}$. 
Minas Gerais-Philander opossum quica

176. Conceição do Mato Dentro, $19^{\circ} 01^{\prime} \mathrm{S}$, $43^{\circ} 25^{\prime} \mathrm{W}$.

177. Lagoa Santa, $19^{\circ} 38^{\prime} \mathrm{S}, 43^{\circ} 53^{\prime} \mathrm{W}$.

P. W. Lund, between 1833 and 1880 .

178. Quartel de Sacramento, $19^{\circ} 44^{\prime} \mathrm{S}, 42^{\circ} 31^{\prime} \mathrm{W}$.

J. Pinto Fonseca, July 1919.

178. Fazenda de Floresta, Rio Matipó, 1953'S, $42^{\circ} 33^{\prime} \mathrm{W}$.

J. Pinto Fonseca, July 1919.

178. Esmeralda (Fazenda), ca. $19^{\circ} 20^{\prime} \mathrm{S}, 42^{\circ} 50^{\prime} \mathrm{W}$.

178. Montes Claras, $19^{\circ} 25^{\prime} \mathrm{S}, 42^{\circ} 35^{\prime} \mathrm{W}$.

179. Boa Esperança, Serra de Caparão, $20^{\circ} 10^{\prime} \mathrm{S}$, $41^{\circ} 46^{\prime} \mathrm{W}$.

E. Kaempfer, August 1929.

180. Fazenda Cardosa, Serra de Caparão, $20^{\circ} 22^{\prime} \mathrm{S}, 41^{\circ} 48^{\prime} \mathrm{W}$.

E. G. Holt, June 1922.

181. Benfica, Serra de Itatiaia, $21^{\circ} 41^{\prime} \mathrm{S}, 43^{\circ} 26^{\prime} \mathrm{W}$.

E. H. Holt, June 1922, at $2000 \mathrm{ft}$.

182. Além Paraíba, $21^{\circ} 52^{\prime} \mathrm{S}, 42^{\circ} 41^{\prime} \mathrm{W}$.

183. Juiz de Fora, $21^{\circ} 45^{\prime} \mathrm{S}, 43^{\circ} 20^{\prime} \mathrm{W}$.

Serviço do Estudos e Pesquisas sôbre a Febra Amarela (SEPSFA).

\section{Espírito Santo-Philander opossum quica}

184. Santa Teresa, $19^{\circ} 55^{\prime} \mathrm{S}, 40^{\circ} 36^{\prime} \mathrm{W}$.

A. M. Olalla, October 1942.

184. São João de Petrópolis, $19^{\circ} 49^{\prime} \mathrm{S}, 40^{\circ} 40^{\prime} \mathrm{W}$.

C. Lako, June 1940.

185. Serra, $20^{\circ} 07^{\prime} \mathrm{S}, 40^{\circ} 18^{\prime} \mathrm{W}$.

C. Lako, September 1949, at $50 \mathrm{~m}$.

185. Campinho, $20^{\circ} 07^{\prime} \mathrm{S}, 40^{\circ} 17^{\prime} \mathrm{W}$.

C. Lako, April 1940, at $500 \mathrm{~m}$.

185. Vitoria, $20^{\circ} 19^{\prime} \mathrm{S}, 40^{\circ} 21^{\prime} \mathrm{W}$.

C. Lako, April 1940.

185. Vila Velha, Morro de Angoles, $20^{\circ} 20^{\prime} \mathrm{S}$, $40^{\circ} 17^{\prime} \mathrm{W}$.

C. Lako, March, April 1940, June 1941.

186. Caixa D'agua, $20^{\circ} 38^{\prime} \mathrm{S}, 40^{\circ} 55^{\prime} \mathrm{W}$.

C. Lako, June 1940.

187. Valão de São Lourenço, Santa Teresa, $20^{\circ} 37^{\prime} \mathrm{S}, 41^{\circ} 41^{\prime} \mathrm{W}$.

C. Lako, June 1940.

188. Engenheiro Reeve (now Rive), $20^{\circ} 46^{\prime} \mathrm{S}$, $41^{\circ} 28^{\prime} \mathrm{W}$.

A. Robert, March, April 1903.
G. R. Hancock, September 1923, at 3000 ft; R. Kellogg, March 1943; G. Perreira, September 1942; D. E. Davis, May, September, October 1943; C. Guinle, Perreira, January 1943; C. Guinle, P. M. Britto, November 1942, March 1943, May 1943; C. Guinle, November 1942; C. Guinle, H. W. Laemmart, April 1943; P. M. Britto, January, May 1943.

189. Boa Vista, Fazenda, Teresópolis (see Teresópolis).

189. Bõa Fé, Fazenda, Teresópolis, $22^{\circ} 22^{\prime} \mathrm{S}$, $41^{\circ} 53^{\prime} \mathrm{W}$.

D. E. Davis, October, September 1943; P. M. Britto, January 1943; G. Perreira, September 1942; C. Guinle, November 1942; C. Guinle, P. M. Britto, March, May 1943; C. Guinle, H. W. Laemmart, April 1943.

189. Novo Friburgo, $22^{\circ} 16^{\prime} \mathrm{S}, 42^{\circ} 32^{\prime} \mathrm{W}$.

Herr Beschke.

189. Barrieira, Serra dos Órgãos, $22^{\circ} 56^{\prime} \mathrm{S}$, $42^{\circ} 56^{\prime} \mathrm{W}$.

Schind in Pohle, 1927.

189. Serra dos Órgãos, $22^{\circ} 56^{\prime} \mathrm{S}, 42^{\circ} 56^{\prime} \mathrm{W}$.

190. Rio de Janeiro, $22^{\circ} 54^{\prime} \mathrm{S}, 43^{\circ} 14^{\prime} \mathrm{W}$.

R. M. Gilmore, January, February 1938.

191. Rodeio, Serra do Mar, $22^{\circ} 33^{\prime} \mathrm{S}, 43^{\circ} 41^{\prime} \mathrm{W}$.

G. B. Flowers.

191. Sepetiba, $22^{\circ} 58^{\prime} \mathrm{S}, 43^{\circ} 42^{\prime} \mathrm{W}$.

J. Natterer, March 1818.

191. Sapitiba (see Sepetiba).

191. São João Marcos, $22^{\circ} 54^{\prime} S, 43^{\circ} 58^{\prime} \mathrm{W}$.

C. Lako, October 1938, January 1939.

192. Mangaratiba, $22^{\circ} 57^{\prime} \mathrm{S}, 44^{\circ} 02^{\prime} \mathrm{W}$.

C. Lako, July, September 1938, February 1939.

193. Itatiaia Parque Nacional, $22^{\circ} 30^{\prime} \mathrm{S}, 44^{\circ} 34^{\prime} \mathrm{W}$. F. Gouvêa, July 1957.

193. Monte Serrat, Parque Nacional de Itatiaya.

C. Moreira, 1901; F. Gouvêa, March 1951, at $800 \mathrm{~m}$, August 1957, at $850 \mathrm{~m}$.

193. Macieiras Itatiaia, $22^{\circ} 30^{\prime} \mathrm{S}, 44^{\circ} 34^{\prime} \mathrm{W}$.

F. Lima, December 1949; F. Gouvêa, December 1949.

193. Barro Branco, $22^{\circ} 23^{\prime} \mathrm{S}, 44^{\circ} 30^{\prime} \mathrm{W}$.

A. Passarell, April 1941.

194. Pedra Branco, Paratí, $23^{\circ} 13^{\prime} \mathrm{S}, 44^{\circ} 43^{\prime} \mathrm{W}$.

C. Lako, July 1943.

São Paulo_Philander opossum quica

195. Avanhandava, Lajeado, $20^{\circ} 57^{\prime} \mathrm{S}, 48^{\circ} 46^{\prime} \mathrm{W}$.

E. Garbe, April 1910.
189. Theresopolis (see Teresópolis).

189. Teresópolis, $22^{\circ} 27^{\prime} \mathrm{S}, 42^{\circ} 57^{\prime} \mathrm{W}$. 
196. Boracéia, upper Rio Tietê, $22^{\circ} 10^{\prime} \mathrm{S}$, $48^{\circ} 45^{\prime} \mathrm{W}$.

J. Pinto, May 1958; L. Travassos, March, April 1958; see also Boracéia (203).

197. Iporanga, Lajeado, $24^{\circ} 36^{\prime} \mathrm{S}, 48^{\circ} 34^{\prime} \mathrm{W}$.

E. Dente, December 1944.

198. Monte Alegre, Amparo, $22^{\circ} 40^{\prime} \mathrm{S}, 46^{\circ} 41^{\prime} \mathrm{W}$.

J. Lima, June 1944.

199. Ypanema $($ Ipanema $=$ Bacaetava $), 23^{\circ} 26^{\prime} \mathrm{S}$. $47^{\circ} 36^{\prime} \mathrm{W}$.

200. São Paulo, $23^{\circ} 30^{\prime} \mathrm{S}, 46^{\circ} 30^{\prime} \mathrm{W}$.

200. Cotia, 12 km W São Paulo (q.v.).

"I.A.L.," January, July 1961, January 1962, November 1963.

200. Cantareira, Serra da, $23^{\circ} 25^{\prime} \mathrm{S}, 46^{\circ} 39^{\prime} \mathrm{W}$.

200. Butantã, Serra da Cantereira (q.v.).

J. Navas, April 1910.

201. Juquia, $24^{\circ} 19^{\prime} \mathrm{S}, 47^{\circ} 36^{\prime} \mathrm{W}$.

201. Costão dos Engenhos, $24^{\circ} 41^{\prime} \mathrm{S}, 4^{\circ} 25^{\prime} \mathrm{W}$.

A. M. Olalla, July 1964.

202. Alto da Serra, $23^{\circ} 47^{\prime} \mathrm{S}, 46^{\circ} 19^{\prime} \mathrm{W}$.

202. Vila Oliveira, Magi das Cruzes, $23^{\circ} 31^{\prime} \mathrm{S}$, $46^{\circ} 11^{\prime} \mathrm{W}$.

J. Lima, September 1943.

203. Salesopolis, Boraceía, $23^{\circ} 32^{\prime} \mathrm{S}, 45^{\circ} 51^{\prime} \mathrm{W}$.

J. Oliveira, June, July 1961.

203. Casa Grande, 20 km S Salesopolis (q.v.).

O. de Sousa Lopez, April 1962, May 1966, May 1968.

203. Boracéia, Ponta de, $23^{\circ} 48^{\prime} \mathrm{S}, 45^{\circ} 49^{\prime} \mathrm{W}$.

Near Salesopolis Biological Station; not to be confused with Boracéia (196).

204. Serra de Itatiaia, $22^{\circ} 55^{\prime} \mathrm{S}, 45^{\circ} 28^{\prime} \mathrm{W}$.

205. Piquete, $22^{\circ} 37^{\prime} \mathrm{S}, 45^{\circ} 10^{\prime} \mathrm{W}$.

Zech, January 1897.

206. São Sebastião, $23^{\circ} 48^{\prime} \mathrm{S}, 45^{\circ} 25^{\prime} \mathrm{W}$.

A. Hempel, July, August 1900.

Paraná-Philander opossum quica

207. Rio Paracaí, $23^{\circ} 41^{\prime} \mathrm{S}, 53^{\circ} 57^{\prime} \mathrm{W}$.

E. Dente, S. Siraglia, January 1954.

208. Roça Nova, Serra do Mar, $25^{\circ} 30^{\prime} S$, $48^{\circ} 50^{\prime} \mathrm{W}$.

A. Robert, Augusi, September 1901.

Santa Catarina-Philander opossum quica

209. Joinville, $26^{\circ} 18^{\prime} \mathrm{S}, 48^{\circ} 50^{\prime} \mathrm{W}$.

E. Steiger, August 1930.

209. Hansa, $26^{\circ} 27^{\prime} \mathrm{S}, 48^{\circ} 50^{\prime} \mathrm{W}$.

W. Ehrhardt, August 1928.
209. Colônia Hansa (see Hansa).

Rio Grande do Sul-Philander opossum quica

210. Passo Fundo, $28^{\circ} 15^{\prime} \mathrm{S}, 52^{\circ} 20^{\prime} \mathrm{W}$.

211. Taquara, $29^{\circ} 31^{\prime} \mathrm{S}, 50^{\circ} 47^{\prime} \mathrm{W}$.

R. Hensel, before 1867; H. von lhering, before 1888 .

\section{Acknowledgments}

My appreciation is expressed for the multitudinous services performed by research assistants Eunice Hoshizaki and Barbara Brown, which contributed substantially to bringing this work to completion, and to Cameron Pfiffner and Kathleen Telfer for the illustrations. The unstinted services essential to this production provided by the Field Museum's photography department, headed by John Weinstein, and the library staff under Benjamin Williams are gratefully acknowledged.

Thanks are expressed to the authorities of the institutions listed below for permission to study the material in their charge.

\section{Literature Cited}

Alho, C. J. R., L. A. Pereira, and A. C. Paula. 1986. Patterns of habitat utilization by small mammal populations in cerrado biome of central Brazil. Mammalia, 5(4): 447-460.

Allen, J. A. 1900. Description of new American marsupials. Bulletin of the American Museum of Natural History, 13: 191-199.

- 1901a. A preliminary study of the North American opossums of the genus Didelphis. Bulletin of the American Museum of Natural History, 14: $149-188$.

___ 1901b. Description of two new opossums of the genus Metachirus. Bulletin of the American Museum of Natural History, 14: 213-218.

1911. Mammals from Venezuela collected by Mr. M. A. Carriker, Jr. Bulletin of the American Museum of Natural History, 30: 239-273.

1912. Mammals from western Colombia. Bulletin of the American Museum of Natural History, 31: $71-95$.

1916a. List of mammals collected for the American Museum in Ecuador by William B. Richardson, 1912-1913. Bulletin of the American Museum of Natural History, 35: 113-125.

1916b. List of mammals collected in Colombia by the American Museum of Natural History Expedition 1910-1915. Bulletin of the American Museum of Natural History, 35: 191-238. 
1916c. Mammals collected on the Roosevelt Brazilian Expedition, with field notes by Leo E. Miller. Bulletin of the American Museum of Natural History, 35: 559-610.

1916d. New South American mammals. Bulletin of the American Museum of Natural History, 35: 83-87.

Alston, E. R. 1879-1882. "Mammalia” in Biologica Centrali-Americana, pp. xxx + 1-220.

ÁlvarEZ, T. 1963. The recent mammals of Tamaulipas, Mexico. Publications, University of Kansas Museum of Natural History, 14(5): 363-473.

Anthony, A. W. 1934. In Goodwin, G. G., Mammals collected by A. W. Anthony in Guatemala 1924-1928. Bulletin of the American Museum of Natural History, 68(1): $1-60$.

Atramentowicz, M. 1986a. Disponibilités trophiques et rhythmes de reproduction chez trois marsupiaux didelphidés de Guyane. Mêmoires Muséum National Histoire Naturelle (Paris), A Zoologie, n.s., 132: 123129.

. 1986b. Dynamique de population chez trois marsupiaux didelphidés de Guyane. Biotropica, 18(2): 136-149.

1988. La frugivorie opportuniste de trois marsupiaux didelphidés de Guyane. Revue Ecologie, 43: 47-57.

Ávil.a-Pires, F. D. DE. 1958. Mamíferos colecionados nos arredores de Belém do Pará. Boletím Museu Paraense Emilio Goeldi, 19: 1-9.

Ávila-Pires, F. D. DE, AND E. Gouvea. 1977. Mamíferos do Parque Nacional do Itatiaia. Boletím Museu Nacional, Rio de Janeiro, Zoology no. 291, 19 pp.

BAER, J. G. 1927. Die Cestoden der Säugethiere Brasiliens. Abhandlungen Senckenbergische Naturforschenden Gesellschaft, 40: 375.

BAKER, R. H. 1951. Mammals from Tamaulipas, Mexico. Publications, University of Kansas Museum of Natural History, 12: 207-218.

. 1974. Records of mammals from Ecuador. Publications, Museum, Michigan State University, Biological Series, 5(2): 131-148.

Belém Virus Laboratory. 1967a. U.S. Public Health Service Publication, 1760: 301-304 (ex Potkay, S. 1977. Diseases of Marsupials). In Hunsaker, D., II, ed., The Biology of Marsupials. Academic Press, New York.

1967b. U.S. Public Health Service Publication, 1760: 593-596 (ex Potkay, S. 1977. Diseases of Marsupials). In Hunsaker, D., II, ed., The Biology of Marsupials. Academic Press, New York.

1967c. U.S. Public Health Service Publication, 1760: 597-600 (ex Potkay, S. 1977. Diseases of Marsupials). In Hunsaker, D., II, ed., The Biology of Marsupials. Academic Press, New York.

Berkovitz, B. K. B. 1978. Tooth ontogeny in Didelphis virginiana (Marsupialia: Didelphidae). Australian Journal of Zoology, 26(1): 61-68.

Bertoni, A. DE W. 1923. Didélfidos de la Bahia de Asunción. Revista Sociedad Científica del Paraguay, 1: 51

1939. Sumario catálogos sistemáticos de los vertebrados del Paraguay. Revista Sociedad Científica del Paraguay, 4(4): 1-60.

BEVEREDGE, I. 1982. Specificity and evolution of the anaplocephalate cestodes of marsupials. Mémoires du Muséum National d'Histoire Naturelle, n.s., Série A, Zoologie, 123: 103-109.

BigGERS, J. D. 1966. Reproduction in male marsupials, pp. 251-277. In Rowlands, 1. W., ed., Comparative Biology of Reproduction in Mammals. Symposium, Zoological Society of London, 15.

BoArdman, W. 1952. The hair tracts of some American marsupials. Proceedings of the Zoological Society of London, 121: 845-850.

BREnNAN, J., AND J. ReID. 1974. The genus Eutrombicula in Venezuela (Acarina: Trombiculidae). Journal of Parasitology, 60(4): 699-711.

Brennan, J. M., And C. E. Yunker. 1966. The chiggers of Panama (Acarina: Trombiculidae), pp. 221-266. In Wenzel, R. L., and V. J. Tipton, eds., Ectoparasites of Panama. Field Museum of Natural History, Chicago.

Brisson, M. J. 1762. Regnum animale in classes IX. Distributum, sine synopsis methodica Quadrupeds. Paris.

Brongniart, A. 1792. Catalogue des mammifères envoyés de Cayenne por M. Le Blond. Actes Societé Histoire Naturelle, Paris, pt. 1: 115.

BufFON, G. L. L. 1763. Histoire naturelle, générale et particulière, avec le description du cabinet du Roi [with account by M. Daubenton]. Imprimerie Royale, Paris, 10: 1-368.

BURMEISTER, H. 1854. Systematische Uebersicht der Thiere Brasiliens, welche wahrend eines Reise durch die Provincen von Rio de Janeiro und Minas Gerais gesammelt oder beobachtet werden. Erster Theil Säugethiere (Mammalia), Berlin, $x+341$ pp.

- 1856. Erläuterungen zur Fauna Braziliens enthaltend Abbildungen und aufühliche Beschreibungen neuer oder ungenügend bekannter Thier-arte. Berlin, viii +115 pp.

Burt, W. H., AND R. A. Stirton. 1961. The mammals of El Salvador. Miscellaneous Publications, Museum of Zoology, University of Michigan, 117: 1-69.

CABAnis, J. L. 1848. Saügethiere, pp. 776-786. In Schomburgk, R., Reisen in Britisch-Guiana in der Jahren 1840-1844, vol. 3. Versuch einer Fauna und Flora im Britisch-Guiana. J. J. Weber, Leipzig.

CABrera, A. 1919. Genera Mammalium, Monotremata, Marsupialia. Museo Nacional de Ciencias Naturales, Madrid, $177 \mathrm{pp}$.

___. 1957[1958]-1961. Catálogo de los mamíferos de America del Sur. Revista del Museo Argentino Ciencias Naturales "Bernardino Rivadavia," Ciencias Zoológicas, 4(1): pp. iv + 1-308. [1958]; 4(2): pp. vxxii + 309-732 [1961].

CARlsson, A. 1903. Beiträge zur Anatomie der Marsupial Region bei den Beutelthieren. Zoologische Jahrbücher, Abteilung Anatomie und Ontogenie der Thiere, 80: 489-505.

CArriker, M. A., JR. 1911. Field notes. In Allen, J. A., Mammals from Venezuela Collected by Mr. M. A. Carriker, Jr., 1909-1911. Bulletin of the American Museum of Natural History, 30(10): 239-273. 
Carvalho, C. T. DE. 1957. Algunas mamíferos do Acre Occidental. Boletím Museu Paraense Emilio Goeldi, 6: $1-22$.

1958. Sobre algunos mamíferos do sudeste do Pará. Arquivos Zoologia, Sảo Paulo, 11(5): 121-132, 1960.

1961. Esboço mastofaunístico do territorio do Rio Branco. Revista Biológica Tropical, 9( I): 1-15.

1962. Lista preliminar dos mamíferos do Amapá. Papeis Avulsos, São Paulo, 15(21): 283-297.

1965. Bionomia de pequeños mamíferos em Boracéia. Revista Biológica Tropical. 13(2): 239-257.

Carvalho, C. T. DE, and A. J. Toccheton. 1969. Mamíferos do nordeste do Pará. Brasil. Revista Biológica Tropical, 15(2): 215-226.

Cerqueira, R., R. Gentile, F. A. S. Fernández, and P. S. L'ANDREA. 1993. A five-year population study of an assemblage of small mammals in southeastern Brazil. Mammalia, 57(4): 499-517.

Charles-Dominique, P. 1983. Ecology and social adaptations in didelphid marsupials: Comparison with eutherians of similar ecology, pp. 395-422. In Eisenberg, J. F., and G. Kleiman, eds., Advances in the Study of Mammalian Behavior. Special Publication 40.7, American Society of Mammalogists.

Charles-Dominique, P., M. Atramentowicz, M. Charles-Dominique, H. Gerard, A. Hladik, C. M. Hladick, AND M. F. Prévost. 1981. Les mammifères frugivorés arboricolés nocturnés d'une foret Guyanaise: Inter-relations plantes-animaux. Revue Ecologie (Terre et Vie), 35: 342-435.

Collins, L. R. 1973. Monotremes and marsupials: A reference for zoological institutions. Smithsonian Institution Publication 4888.

CORRéa Gomes, D. 1984. Philander opossum (Marsupialia), a new host of record for Sparganum of Lueheella Baer, 1924 (= Spirometra Mueller, 1937). Memórias Instituto Oswaldo Cruz, 79(3): 369-370.

Costa Lima, A. da, and C. Hathaway. 1946. Pulgas. Bibliografía, catálogo e hospedadores. Monografías do Instituto Oswaldo Cruz no. 4 (Rio de Janeiro), pp. $1-522$.

COWPER, W. 1704. An account of the anatomy of those parts of a male opossum that differ from the female. Philosophical Transactions of the Royal Society of London, 5: 1576.

Creighton, G. K. 1984. Systematic studies on opossums (Didelphidae) and rodents (Cricetidae). Unpublished dissertation. University of Michigan, Ann Arbor.

Crespo, J. A. 1950. Nota sobre mamíferos de Misiones nuevos para Argentina. Communicaciones Instituto Nacional Investigaciones Ciencias Naturales, Museo Argentino Ciencias Naturales "Bernardino Rivadavia," Ciencias Zoológicas, 1(14): 1-14.

1974. Comentarios sobre nuevos localides para mamíferos de Argentina y de Bolivia. Revista Museo Argentino Ciencias Naturales "Bernardino Rivadavia," Ciencias Zoologícas, 11(1): 1-31.

Cuvier, G. 1798. Tableau elementaire d'histoire naturelle des animaux. Baudoin, Paris.

Dalquest, W. W. 1953. Mammals of the Mexican State of San Luis Potosí. Louisiana State University Studies, Biological Sciences Series, 1: 1-122.

DAvIS, D. E. 1945a. The home range of some Brazilian mammals. Journal of Mammalogy, 26(12): 119-127.

1945b. The annual cycle of plants, mosquitos, birds, and mammals in two Brazilian forests. Ecological Monographs, 15: 243-295.

1947. Notes on the life histories of some Brazilian mammals. Boletím Museu Nacional (Rio de Janeiro), no. 76: 1-8.

DeAne, L. M. 1961. Tripanosomídeos de mamíferos da região Amazônica: 1. Alguns flagelados encontrados no sangue de mamíferos silvestres do Estado do Pará. Revista Instituto de Medicina Tropical, São Paulo, 3: 15-28. (ex Potkay, S., in Hunsaker, D., II, ed., 1977).

1964. Tripanosomídeos de mamíferos da região Amazônica: III. Hemoscopia e xenodiagnóstico de animais silvestres dos arredores de Belém, Pará. Revista Instituto de Medicina Tropical, São Paulo, 6: 225-232. (ex Potkay, S., in Hunsaker, D., II, ed. 1977).

Desmarest, A. G. I820. Mammalogie ou description des espèces de mammifères. Premiére parte, pp. viii $+1-276,1820$. Seconde parte, viii $+277-555,1822$. Paris.

1827. Article Sarigüe, Dictionaire de Science Naturelle, 47: 377-400. Paris.

Dubost, G. 1971. Personal communication, p. 197. In Charles-Dominique, P., Ecoethologie des prosimiens du Gabon. Biologia Gabonica, 7: 121-228.

EisenberG, J. F., AND D. E. Wilson. 1981. Relative brain size and demographic strategies in didelphid marsupials. American Naturalist, 118(1): 1-15.

Emmons, L. H., AND F. FEer. 1990. Neotropical Rainforest Mammals: A Field Guide. University of Chicago Press, Chicago, 281 pp.

Enders, A. C., And R. K. Enders. 1969. The placenta of the four-eyed opossum (Philander opossum). Anatomical Record, 165(3): 431-450.

ENDERS, R. K. 1935. Mammalian life histories from Barro Colorado Island, Panama. Bulletin of the $\mathrm{Mu}$ seum of Comparative Zoology, 78(4): 385-502.

- 1937. Panniculus carnosus and formation of the pouch in Didelphis. Journal of Morphology, 61(1): $1-26$.

. 1966. Attachment, nursing, and survival of young in some didelphids, pp. 195-203. In Rowlands, 1. W., ed., Comparative Biology of Reproduction in Mammals. Symposium of the Zoological Society of London, 15: 195-203.

ENDERS, R. K., AND D. E. DAVIS. 1936, Body temperatures of several Central American mammals. Journal of Mammalogy, 17: 165-166.

Engstrom, M. D., And A. J. Gardner. 1988. Karyotype of Marmosa canescens (Marsupialia: Didelphidae): A mouse opossum with 22 chromosomes. Southwestern Naturalist, 33(2): 231-233.

Fairchild, G. B., G. M. Kohls, AND V. J. Tipton. 1966. The ticks of Panama (Acarina:Ixodoidea), pp. 167219. In Wenzel, R. L., and V. J. Tipton, eds., Ectoparasites of Panama. Field Museum of Natural History, Chicago. 
FARRIS, E. J. 1950. The opossum, pp. 256-267. In Farris, E. J., ed., The Care and Feeding of Laboratory Animals. John Wiley \& Sons Inc., New York.

Felton, H. 1958. Weitere Säugetiere aus El Salvador (Mammalia: Marsupialia, Insectivora, Primates, Edentata, Lagomorpha, Carnivora und Artiodactyla). Senckenbergiana Biologica, 39(5/6): 213-228.

FLEMING, T. H. 1972. Aspects of the population dynamics of three species of opossums in the Panama Canal Zone. Journal of Mammalogy, 53(3): 619-623.

. 1973. The reproductive cycles of three species of opossums and other mammals in the Panama Canal Zone. Journal of Mammalogy, 54(2): 439-455.

Fonseca, G. A. B. DA, And M. C. M. KierulfF. 1989. Biology and natural history of Brazilian Atlantic forest small mammals. Bulletin of the Florida State Museum, 34(3-4): 1-157.

Fonseca, G. A. B. DA, And K. H. RedFord. 1984. The mammals of IBGE's ecological reserve, Brasília, and an analysis of the role of gallery forests in increasing diversity. Revista Brasileira Biologia, 44(4): 517-523.

Furman, D. 1972. Laelapid mites (Laelapidae: Laelapinae) of Venezuela. Brigham Young University Scientific Bulletin, Biological Series, 17(3): 1-58.

Gardner, A. L. 1973. The systematics of the genus Didelphis (Marsupialia: Didelphidae) in North and Middle America. Special Publication, Museum of Texas Tech University, 4: 1-81.

Journal of Mammalogy, 62(2): 445-448.

1993. Order Didelphimorphia, pp. 15-23. In Wilson, D. E., and D. M. Reeder, eds., Mammal Species of the World. A Taxonomic and Geographic Reference, 2nd edition. Smithsonian Institution Press, Washington, D.C.

Gardner, A. L., And J. L. Patton. 1972. New species of Philander (Marsupialia: Didelphidae) and Mimon (Chiroptera: Phyllostomidae) from Peru. Occasional Papers, Louisiana State University Museum of Zoology, 43: 1-12.

Geoffroy, É. 1803. Catalogue des Mammifères du Muséu National de Histoire Naturelle. Paris, 272 pp.

Giebel, C. G. 1859. Die Naturgeschichte des Theirreichs. Erster Band. Die Säugethiere. Leipzig, pp. viii +522 .

Gilmore, R. M. 1941. The susceptibility to yellow fever of the vertebrates of eastern Colombia I. Marsupialia. American Journal of Tropical Medicine, 21: 309-333.

Goeldi, E. A. 1894. Critical gleanings on the Didelphidae of the Serra dos Orgãos, Brazil. Proceedings of the Zoological Society of London, 1894: 457-466.

Goeldi, E. A., and Hagmann, G. 1904. Prodromo de um catálogo crítico-commentado da coleção de mammíferos no Museu do Pará (1894-1903). Boletim Museu Paraense (A) Zoologica, 4: 38-122.

GoldFuss, G. A. 1809. Vergleichende Naturbeschreibung der Säugethiere. Erlangen.

Goldman, E. A. 1920. Mammals of Panama. Smithsonian Miscellaneous Collections, 69(5): 1-309.

Goodwin, G. G. 1934. Mammals collected by A. W.
Anthony in Guatemala 1924-1928. Bulletin of the American Museum of Natural History, 68: 1-60.

- 1942. Mammals of Honduras. Bulletin of the American Museum of Natural History, 79: 107-195.

- 1946. Mammals of Costa Rica. Bulletin of the American Museum of Natural History, 87(5): 271474.

- 1953. Catalogue of type specimens of recent mammals in the American Museum of Natural History. Bulletin of the American Museum of Natural History, 102(3): 211-411.

GRAY, J. E. 1821. On the natural arrangement of vertebrose animals. London Medical Repository, Monthly Journal, and Review, 15: 296-311.

- 1843. List of the Specimens of Mammalia in the Collection of the British Museum, London.

Grayson, M. A., And P. Galindo. 1968. American Journal of Epidemiology, 88: 80.

Gronovius, L. T. 1763. Gronoviani fascicle primus. Lyons. fol.

Guerrero, R. 1983. Nematoda: Trichostrongloidea parásitos de mamíferos silvestres de Venezuela. 11. Revisión del Género Viannaia Travassos, 1914. Memorias Sociedad Ciéncias Naturales La Salle, (Venezuela), $1-119$.

- 1985. Parasitología, pp. 35-91. In Aguilera, M., ed., El Estudio de los Mamíferos en Venezuela. Evaluación y Perspectivas. Asociación Venezolana para el Estudio de los Mamíferos (ASOVEM), Carácas.

HAFFER, J. 1969. Speciation in Amazonian forest birds. Science, 165: 131-137.

Hall, E. R., and W. W. Dalquest. 1963. The mammals of Veracruz. Publications, University of Kansas Museum of Natural History, 14(4): 165-362.

Hall, E. R., and K. R. Kelson. 1959. The Mammals of North America, vol. 1. Ronald Press Company, New York. 1: 1-546; 2: 247-1073 + 79 .

Handley, C. O. 1976. Mammals of the Smithsonian Venezuelan Project. Brigham Young University Science Bulletin, Zoological Series, 20(5): 1-89.

Hartman, C. G. 1920. Studies in the development of the opossum Didelphys virginiana $\mathrm{L}$. V. The phenomena of parturition. Anatomical Record, 19: 251-261.

Hayman, D. L., and P. G. Martin. 1969. Cytogenetics of marsupials, pp. 191-217. In Benirschke, K., ed., Comparative Mammalian Cytogenetics. Springer-Verlag, New York.

HENSEL, R. 1872. Beiträge zur kenntniss der Säugethiere Süd-Brasiliens. Abhandlungen Berlin Akademie der Wissenschaften, Berlin, 1872: 120-130.

Hershkovitz, P. 1941. The South American harvest mice of the genus Reithrodontomys. Occasional Papers, Museum of Zoology, University of Michigan, 441: 1-7.

1949. Generic names of the four-eyed pouch opossum and the woolly opossum (Didelphidae). Proceedings of the Biological Society of Washington, 62: $11-12$.

1959. Nomenclature and taxonomy of the neotropical mammals described by Olfers, 1818. Journal of Mammalogy, 40(3): 337-353. 
1968. Metachromism or the principle of evolutionary change in mammalian tegumentary colors. Evolution, 22(3): 556-575.

1970. Metachromism like it is. Evolution, 24(3): 644-648.

1976. Comments on generic names of foureyed opossums (family Didelphidae). Proceedings of the Biological Society of Washington, 89(23): 295304.

1977. Living New World Monkeys (Platymhini) with an Introduction to Primates, vol. 1. University of Chicago Press, Chicago.

1981. Philander and four-eyed opossums once again. Proceedings of the Biological Society of Washington, 93(4): 943-946.

1992a. The South American gracile mouse opossums, genus Gracilinamus Gardner and Creighton, 1989 (Marmosidae, Marsupialia): A taxonomic review with notes on general morphology and relationships. Fieldiana: Zoology, n.s., 70: 1-56.

1992b. Ankle bones: The Chilean opossum Dromiciops gliroides Thomas, and marsupial phylogeny. Bonner Zoologische Beiträge, 43(2): 181-213.

1995. The staggered marsupial lower incisor: Hallmark of cohort Didelphimorphia, and description of a new genus and species with $i_{3}$ from the Albian (Lower Cretaceous) of Texas. Bonner Zoologische Beiträge, 45(3-4): 153-169.

- 1996. Dromiciops gliroides Thomas, 1894: Last of the Microbiotheria (Marsupialia), with a review of the family Microbiotheriidae. Fieldiana: Zoology, in press.

HILDEBRAND, M. 1961. Body proportions of didelphid (and some other) marsupials, with emphasis on variability. American Journal of Anatomy, 109(3): 239249.

HiLl, J. P., and E. A. Fraser. 1925. Some observations on the female urogenital organs of the Didelphyidae. Proceedings of the Zoological Society of London, 1925: 189-219.

HochstetTer, F. 1946. Über die Karte Hirnhaut und ihre Fortsätze bei den Säugethieren. Nebst Angebene über die Sagereziehung der Einzelnen Hirnteile dieser Tiere zu einander, zu der Fortsätzen der karten Hirnhaut und zur Schädelkapsel. Denkschriften Academie Wissenschaffen Wien, Mathematisch-naturwissenschaftliche Klasse, 106(2): 144 pp.

Honacki, J. H., K. E. Kinman, AND J. W. Koeppel, eds. 1982. Mammal Species of the World: A Taxonomic and Geographic Reference. Allen Press, Inc., and the Association of Systematic Collections, Lawrence. Kansas, 694 pp.

Hopkins, G. H. E. 1949. Host associations of the lice of mammals. Proceedings of the Zoological Society of London, 119(1949-1950): 387-607.

Hopwood, A. T. 1947. The generic names of the mandrill and baboons, with notes on some of the genera of Brisson, 1762. Proceedings of the Zoological Society of London, 117: 533-536.

Hunsaker, D., and D. Shupe. 1977. Behavior of New World marsupials, pp. 279-347. In Hunsaker, D., II, ed., The Biology of Marsupials. Academic Press, New York.
Husson, A. M. 1978. Mammals of Suriname. E. J. Brill, Leiden, 569 pp.

Hutterer, R., M. VerhaAgh, J. Diller, and R. PodLOUCKY. 1995. An inventory of mammals observed at Panguana Biological Station, Amazonian Peru. Ecotropica, 1(1): 3-20.

IHERING, H. vON. 1892. Os mammiferos do Rio Grande do Sul, pp. 99-123. Annuario do Estado do Rio Grande do Sul para 1893, publicado por Graciano A. de Arambuja, v. IX, Porto Alegre, 1892.

— 1894. Os mammíferos de São Paulo, Catálogo "Diario Official," pp. 3-30.

ILliger, C. VON, 1815. Ueberblick der Säugthiere nach ihrer Vertheilung über die Weltheile. Abhandlungen Königlich Akademie Wissenschaft Berlin (18041811). pp. 39-159.

Imperial Bureau of Agricultural, Parasitology. 1933. Journal of Helminthology. 11:195. (ex Potkay. S. 1977. Diseases of Marsupials). In Hunsaker, D., II, ed., The Biology of Marsupials. Academic Press, New York.

INTERNATIONAL COMmISSION ON ZOOLOGICAL NOMENCLATURE. 1961 (revised 1964). International Code of Zoological Nomenclature. Published for the International Commission on Zoological Nomenclature by the International Trust for Zoological Nomenclature, London. $x x+176$ pp. Editors: N. R. Stoll (Chairman), R. Ph. Dollfus, J. Forest, N. D. Riley, C. W. Sabrowsky, C. W. Wright, R. V. Melville (Secretary).

INTERNATIONAL COMMISSION ON ZOOLOGICAL NOMENCLATURE. 1985. International Code of Zoolugical Nomenclature. Published for the International Commission on Zoological Nomenclature by the International Trust for Zoological Nomenclarure. London.

JENKINS, D., AND L. KNUTSON. 1983. A catalogue of the type specimens of Monotremata and Marsupialia in the British Museum (Natural History), British Museum (Natural History), London, 1983: 1-34.

Jentink, F. A. 1887. Catalogue Osteologique des mammiferes. Muséum Histoire Naturelle Pays-Bas, Zoologie, 9: I-360.

- 1888. Catalogue systématique des mammiferes (rongeurs, insectivores, cheiroptères, édéntes et marsupiaux). Muséum Histoire Naturelle Pays-Bas, 12: 1280.

Jones, E., C. Clifford, J. Kierens, and G. Kohls. 1972. The ticks of Venezuela (Acarina: Ixodoidea) with a key to the species of Amblyomina in the Western Hemisphere. Brigham Young University Scientific Bulletin, Biological Series, 17(4): 1-40.

Kantis, E. 1963. Nueva localidad para marsupiales didélfidos coleccionados en el Chaco. Neotropica (La Plata. Argentina), 9(29): 54.

KirSCH, J. A. W. 1977. The comparative serology of Marsupialia, and a classification of marsupials. Australian Journal of Zoology, Supplement Series, 52: 1152.

Kirsch, J. A. W., R. E. Bleiweiss, A. W. Dickerman, AND O. A. REIG. 1993. DNA/DNA hybridization studies of carnivorous marsupials. III. Relationships among species of Didelphis (Didelphidae). Journal of Mammalian Evolution, 2: 75-97.

Kirsch, J. A. W., A. W. Dickerman, and O. A. Reig. 
1993. DNA/DNA hybridization studies of carnivorous marsupials. IV. Intergeneric relationships among opossums (Marsupialia: Didelphidae). Marmosiana, 1.

Kirsch, J. A. W., AND R. E. Palma. 1995. Hybridization studies of carnivorous marsupials. V: A further estimate of relationships among opossums (Marsupialia, Didelphidae). Mammalia, 59(3): 403-425.

Kirsch, J. A. W., AND P. E. Waller. 1979. Notes on the trapping and behavior of the Caenolestidae (Marsupialia). Journal of Mammalogy, 60(2): 390-395.

Kishino, H., AND M. Hasegawa. 1989. Evaluation of the maximum likelihood estimate of the evolutionary tree topologies from DNA sequence data, and the branching order in Hominoidea. Journal of Molecular Evolution, 29: 170-179.

KRIEG, H. 1924. Beobachtungen an argentinischen Beutelratten. Zeitschrift für Morphologie und Ökologie der Tiere, 1: 637-659.

Krumbiegel, I. 1941. Die Säugetiere der SudamerikaExpeditionen Prof. Dr. Kriegs. 11. Mittelgrosse Didelphyiden (Lutreolina u. Metachirus). Zoologischer Anzeiger, 134(9/10): 190-211.

Laemmert, H. W., JR. 1967. U.S. Public Health Service Publication 1760, pp. 377-380 (ex Potkay, S. 1977. Diseases of Marsupials). In Hunsaker, D., II, ed., The Biology of Marsupials. Academic Press, New York.

Laemmert, H. W., JR., L. DE C. Ferreira, and R. M. TAYloR. 1946. American Journal of Tropical Medicine, 26: 23 (ex Potkay, S. 1977. Diseases of Marsupials). In Hunsaker, D., II, ed., The Biology of Marsupials. Academic Press, New York.

Lainson, R., AND J. J. Shaw. 1969. Transactions of the Royal Society of Tropical Medicine Hygiene, 63: 408, 738 (ex Potkay, S. 1977. Diseases of Marsupials). In Hunsaker, D., II, ed., The Biology of Marsupials. Academic Press, New York.

LANDSMEER, J. M. F. 1979. The extensor assembly in two species of opossum, Philander opossum and Didelphis marsupialis. Journal of Morphology, 161(3): 337-346.

LAPOINTE, F.-J., J. A. W. KiRSCH, AND R. Bleiweiss. 1994. Jackknifing of weighted trees: Validation of phylogenies reconstructed from distance matrices. Molecular and Phylogenetic Evolution, 3: 256-267.

LAtreille, P. A. 1803. Exposition méthodique des quadrupèdes specialement mentiones dans cette edition de l'Histoire Naturelle de Buffon. Histoire $\mathrm{Na}$ turelle, Générale et Particulíere, Des Quadrupèdes, vol. 34: 251-321. Sonnini ed., Paris, An XI (1803).

LAYNE, J. N. 1951. The use of the tail by an opossum. Journal of Mammalogy, 32(4): 464-465.

LiAIS, E. 1872. Climates, géologie, faune et géographie botanique du Brésil. Garnier Frères, Paris.

Linnaeus, C. 1758. Systema Naturae, I., Regnum Animalium, ed. 10, Reformed, Holmia.

LÖNNBERG, E. 1913. Mammals from Ecuador and related forms. Arkiv för Zoologie, Stockholm, 8(16): 136.

1921. A second contribution to the mammalogy of Ecuador with some remarks on Caenolestes. Arkiv för Zoologie, Stockholm, 14(4): 1-104.

LUCKETT, W. P. 1993. An ontogenetic assessment of dental homologies in therian mammals, pp. 182-204. In Szalay, F. S., M. J. Novacek, and M. C. McKenna, eds., Mammal Phylogeny. Springer-Verlag, New York.

LYDEKKER, R. 1896. A Handbook to the Marsupialia and Monotremata, Lloyd's Natural History (London), 320 pp.

LYNE, A. G. 1959. The systematic and adaptive significance of the vibrissae in the Marsupialia. Proceedings of the Zoological Society of London, 133(1): 79-132.

Mares, M. A., M. R. Willig, And T. E. LAcher, JR. 1985. The Brazilian caatinga in South American zoogeography: Tropical mammals in a dry region. Journal of Biogeography, 12: 57-69.

Mares, M. A., J. K. Braun, and D. D. Gettinger. 1989. Observations on the distribution and ecology of the mammals of the cerrado grasslands of central Brazil. Annals of the Carnegie Museum, 58(1): 1-60.

Marshall, L. G., AND C. DE Muizon. 1988. The dawn of the age of mammals in South America. National Geographic Research, 4(1): 23-55.

Marshall, L. G., C. de Muizon, and D. SigogneauRussell. 1995. Pucadelphys andinus (Marsupialia, Mammalia) from the early Paleocene of Bolivia. Mémoires du Muséum National D'Histoire Naturelle (Paleontologie), 165: 1-164.

Matschie, P. 1916. Bemerkungen über die Gattung $D i$ delphis L. Sitzungsberichte Gesellschaft Naturforschunde Freunde, Berlin, 1916: 259-272.

MCCRADY, E., JR. 1938. The Embryology of the Opossum. Wistar Institute of Anatomy and Biology, Philadelphia, $226 \mathrm{pp}$.

MCNAB, B. K. 1978. The comparative energetics of neotropical marsupials. Journal of Comparative Physiology, 125: 115-128.

Mello, D. A., AND L. E. Moojen. 1979. Nota sobre uma coleção de roedores e marsupias de algumas regiões do cerrado do Brasil central. Revista Brasileira Pesquisas Medicas e Biologicas, 12(4-5): 287-291.

MÉNDEZ, E. 1972. A new fur mite of the genus Archemyobia parasitic on Philander opossum fuscogriseus (Acarina: Myobidae). Acaralogia, 13(4): 615-620.

1977. Mammalian-siphonapteran associations, the environment, and biogeography of mammals of southwestern Colombia. Questiones Entomologicae, 13: $91-182$.

MÉnÉgauX, M. A. 1902. Catalogue des Mammifères rapporté por M. Geay de la Guyane Française en 1898 et 1900. Bulletin Muséu National Histoire Naturelle, Paris, 8: 490-496.

Miles, M. A., A. A. DE Souza, And M. M.PóvoA. 1981a. Mammal tracking and nest location in Brazilian forest with an improved spool-and-line device. Journal of Zoology, 195(3): 331-348.

1981b. Chaga's disease in the Amazon basin. III. Ecotopes of ten Ariatomine bug species (Hermiptera: Reduviidae) from the vicinity of Belém, Pará state, Brazil. Journal of Medical Entomology, 18(4): 266-278.

Miller, L. E. 1916. Mammals collected on the Roosevelt Brazilian Expedition, with field notes by Leo E. Miller. In Allen, J. A. Bulletin of the American Museum of Natural History, 35(30): 589-610. 
MiRANDA Ribeiro, A. DE. 1905. Vertebrados do Itatiaya (peixes, serpentes, saurios, aves e mammiferos) resultados do exursões do $\mathrm{Sr}$. Carlos Moreira, assistente da Secçāo de Zoologia do Museu Nacional. Arquivos Museu Nacional (Rio de Janeiro), pp. 163-190.

1936. Didelphia ou Mammalia-ovovipara marsupiaes, didelphos, pedimanos ou Metaterios. Revista do Museu Paulista, São Paulo, 20: 246-427.

Mondolfi, E., and G. Medina Padilla. 1958. Contribución al conocimiento del "perrito de agua." Memoria de la Sociedad de Ciencias Naturales La Salle, 17(48): $141-155$.

MoOJEn, J., And F. D. Ávilla-Pires. 1966. Observacōes gerais sôbre a mastozoologia do cerrado. Annales Academia Brasileira Ciencias, 38(supplemento): 33 l340 .

MuirheAD, D. 1830. Brewster's Edinburgh Encyclopedia, vol. 13, p. 429.

Murie, A. 1935. Mammals from Guatemala and British Honduras. Miscellaneous Publications, University of Michigan Museum of Zoology, 26: 1-30

Mustrang, M. A. 1994. Marmosops scapulatus Burmeister, 1856, and the brown mutation in didelphids (Marsupialia). Zeitschrift für Säugetierkunde, 59: 252-254.

O'Connell, M. A. 1979. Ecology of didelphid marsupials from northern Venezuela, pp. 73-87. In Eisenberg, J. F., ed., Vertebrate Ecology in the Northern Neotropics. Smithsonian Institution Press, Washington, D.C

OLFERS, I. F. J. 1818. Bemerkungen zu llliger's Ueberblick der Saügthiere nach ihrer Vertheilung über die Welttheile, rücksichtlich der Südamericanischen Arten (Species). Abhandlungen, In W. L. Eschwege, Journal von Brasilien, 15(2): 192-237. (Neue Bibliothek der wichtigsten Reisebeschreibungen zur Erweiterung der Erd-und Volkerkunde.) K. T. Bertuch, Weimar.

Osgood, W. H. 1913. New Peruvian mammals. Field Museum of Natural History Zoological Series, 10(9): 93-100.

1914. Mammals of an expedition across northern Peru. Field Museum of Natural History Zoological Series, 10: 143-185.

1921. A monographic study of the American marsupial, Caenolestes. Field Museum of Natural History Zoological Series, 14(1): 1-56.

Oudemans, J. T. 1892. Die accessorischen Geschlectsdrüsen der Säugthiere. Naturkundlige Verhandlungen Hollandische Maatschappij der Wetenschappen, (3) 5(2): l-96

Patton, J. L., S. F. Dos Reis, and M. N. dA Selva. 1995 [1996]. Relationships among didelphid marsupials based on sequence variation in the mitochondrial cytochrome B gene. Journal of Mammalian Evolution, 3(1): 3-29.

PElzELN, A. von. 1883. Brazilische Säugethiere: Resultate von Johann Natterer's Reisen in den Jahren 1817 bis 1835 . Verhandlungen Kaiserliche Königliche Zoologischbotanischen Gesellschaft, Wien Beiheft, 33: $1-140$.

Pérez-Hernández, R. 1985. Notas preliminares acerca de la taxonomía de la familia Didelphidae (Mamma- lia: Marsupialia) en Venezuela. Memorias de la Sociedad Ciencias Naturales La Salle, 45(I23): 47-76. 1989 [1990]. Distribution of the family Didelphidae (Mammalia: Marsupialia) in Venezuela, pp. 363-410. In Redford, R. H., and J. F. Eisenberg, eds., Advances in Neotropical Mammalogy. Sandhill Crane Press, Gainesville, Florida.

Pérez-Hernández, R., R. Bodini, H. J. Finol, and V. GOMEZ. 1986. Contribución al estudio de la morfología dentaria de los didélfidos (Mammalia-Marsupialia). Secundas Jorñadas de Microscopia Electrónica 14-17 Julio, 1986, Facultad de Ciencias Centro de Microscopia Electrónica, UCV Caracas, Venezuela.

Perondini, A. L. P., AND D. R. PERondini. 1965. Analise comparativa dos cromosomos somáticos de quatro especies de marsupiais. Revista Brasileira Biologia (Rio de Janeiro), 25(4): 377-386.

Perondini, A. L. P., and D. R. Perondini. 1966. Sex chromosomes in somatic cells of the Philander opos. sum quica (Temminck, 1827, Marsupialia). Cytogenetics, 5: 28

Phillips, C. J., And J. K. Jones, JR. 1969. Notes on reproduction and development in the four-eyed opossums, Philander opossum, in Nicaragua. Journal of Mammalogy, 50(2): 345-348.

PINE, R. H. 1973. Anatomical and nomenclatural notes on opossums. Proceedings of the Biological Society of Washington, 86(3): 391-402.

Pine, R. H. 1973. Mammals (exclusive of bats) of Belém, Pará, Brazil. Acta Amazonica, 3(2): 47-49.

Pohle, H. 1927. Ueber die von Prof. Breslau in Brasilien gesammelten Säugethiere. Abhandlungen Senckenbergische Naturforschende Gesellschaft, 40: 239249.

Potkay, S. 1977. Diseases of Marsupials, pp. 415-506. In Hunsaker, D., II, ed., The Biology of Marsupials. Academic Press, New York.

PRANCE, G. T. 1982. Forest refuges: Evidence from woody angiosperms, pp. 137-157. In Prance, G. T. ed., Biological Diversification in the Tropics. Columbia University Press, New York.

PRAY, L. L. 1921. Opossum carries leaves with its tail. Journal of Mammalogy, 2(2): 109-1 10.

RedFord, K. H., AND G. A. B. DA Fonseca. 1986. The role of gallery forests in the zoogeography of the cerrado's non-rodent mammalian fauna. Biotropica, 18(2): $126-135$.

RLgendanz, R., AND W. KikUth. 1928. Sur un parasite du sang des "Quica" (Metachirus quica), Nuttalia brasiliensis $\mathrm{n}$. sp., et influence de la rate sur les infections latentes du sang. Comptes Rendus Societé Biologie, Paris, 98: 1567.

Reig, O. A., A. L. Gardner, N. O. Bianchi, and J. L. PatTon. 1977. The chromosomes of the Didelphidae (Marsupialia) and their evolutionary significance. Biological Journal of the Linnaean Society, 9(12): 191216.

Reig, O. A., J. A. W. Kirsch, And L. Marshall. 1985. New conclusions on the relationships of the opossumlike marsupials, with an annotated classification of the Didelphimorphia. Ameghiniana, 21(2-4): 335-343.

Rejg, O. A., J. A. W. Kirsch, and L. G. Marshall. 
1987. Systematic relationships of the living and Neocenozoic American "opossum-like" marsupials (suborder Didelphimorphia), with comments on the classification of these and of the Cretaceous and Paleogene New World and European metherians, pp. 1-89. In Archer, M., ed., Possums and Opossums: Studies in Evolution. Surrey Beatty and Sons, Chipping Norton, New South Wales.

REYNOLDS, H. C. 1952. Studies on reproduction in the opossum (Didelphis virginiana). University of California Publications in Zoölogy, 52(3): 223-284.

Rodger, J. C. 1982. The testis and its excurrent ducts in American caenolestid and didelphid marsupials. American Journal of Anatomy, 163: 269-282.

Ruschi, A. 1965. Lista dos mamíferos do Estado do Espírito Santo. Boletím Museo Biología Prof. Mello Leitão, Zoología, 24a(2): 1-48.

SANBORN, C. C. 1947. Catalogue of type specimens of mammals in Chicago Natural History Museum. Fieldiana: Zoology 32(4): 209-293.

1949. Mammals from the Río Ucayali, Peru. Journal of Mammalogy, 30: 277-288.

. 1951. Mammals from Marcapata, southeastern Peru. Museo Historia Natural "Javier Prado," Universidad Nacional Mayor San Marcos, 6: 1-21.

SANDERSON, 1. T. 1949. A brief review of the mammals of Suriname (Dutch Guiana), based on a collection made in 1938. Proceedings of the Zoological Society of London, 119(3): 755-789.

Schinz, H. R. 1844. Systematische Verzeichnung aller bis jetzt bekannten Säugethiere. Synopsis Mammalium nach dem Cuvierschen System, vol. 1. Jent und Gassmann, Solothurn, pp. xvi-587.

SCHIRCH, P. F. 1932. Contribução ao conhecimento do Fauna da Serra dos Orgãos. Boletín Museu Nacional, Rio de Janeiro, 8: 77-86.

Schomburgk, R. 1840. Expedition in Guiana. Annals of Natural History, 5: 343-348.

SchomburGK, R. 1848. Versuch einer Fauna und Flora von Britisch-Guayana. Vol. 3 of Reisen in Britisch Guiana. Leipzig, Weber, pp. x, 533-1261.

Schreber, J. C. D. von. 1739-1810. Die Säugethiere in Abbildungen nach der Natur, mit Beschreibungen. Leipzig.

SEBA, A. 1734. Locupletissimi rerum naturalium thesauri accurata descriptio et iconibus artificiosissimis expressio, per universam physices historiam opus. . . Amsterdam.

SeEvers, C. H. 1955. A revision of the tribe Amblyopinini: Staphalinid beetles parasitic on mammals. Fieldiana: Zoology, 37: 211-264.

Shaw, G. 1800. General Zoology-Mammalia 1(2). G. Kearsley, London.

Shope, R. E. I967a. U.S. Public Health Service Publication 1760, pp. 293-296, (ex Potkay, S. 1977. Diseases of Marsupials). In Hunsaker, D., II, ed., The Biology of Marsupials. Academic Press, New York.

SonntaG, C. F. 1924. The comparative anatomy of the tongues of the Mammalia. XI. Marsupialia and Monotremata. Proceedings of the Zoological Society of London, 49: 743-755.

Tantaléan, J. M., L. Sarmiento B., and F. A. Huiza.
1992. Digeneos (Trematoda) del Peru. Boletín de Lima, 14(80): 47-84.

TATE, G. H. H. 1939. The mammals of the Guiana region. Bulletin of the American Museum of Natural History, 76: 151-229.

TAYloR, D. L., AND M. H. Shacklette. 1962. American Journal of Tropical Medicine, 11: 796 (ex Potkay, S. 1977. Diseases of Marsupials). In Hunsaker, D., II, ed., The Biology of Marsupials. Academic Press, New York.

Temminck, C. J. 1824-1827. Monographies de Mammalogie ou description de quelques génres de mammifères, dont les espécies ont été observées dans les differents musées de l'Europe. Deuxième monographie sur le genre Sariguie-Didelphis (Linn), Paris, 1: 21-54.

TEMminck, C. J. 1827. Deuxième monographie sur le genre Sarigue (1)—Didelphis. (Linn.), pp. 21-72, 9 pls. In Temminck, C. J., ed., Monographies de Mammalogie. Paris, G. Dufour et Ed. D'Ocagne.

Tesh, R. B., P. H. Peralta, and K. M. Johnson. 1969. American Journal of Epidemiology, 90: 255 (ex Potkay, S. 1977. Diseases of Marsupials). In Hunsaker, D., II, ed., The Biology of Marsupials. Academic Press, New York.

Thomas, O. 1888. Catalogue of the Marsupialia and Monotremata in the collection of the British Museum (Natural History). British Museum (Natural History), London.

1899. Descriptions of new neotropical mammals. Annals and Magazine of Natural History, (7) 4: 278-288.

- 1901a. New Myotis, Artibeus, Sylvilagus, and Metachirus from Central and South America. Annals and Magazine of Natural History, (7) 7: 541-545.

- 1901b. On a collection of mammals from the Kanuku Mountains, British Guiana. Annals and Magazine of Natural History, (7) 8: 139-154.

ThOMAS, O. 1902a. On mammals from the Serra do Mar of Paraná, collected by Mr. Alphonse Robert. Annals and Magazine of Natural History, (7) 9: 59-64.

- 1902b. Mammals from Cochabamba, Bolivia, and the region north of that place. Annals and Magazine of Natural History, (7) 9: 125-143.

1910. Mammals from the River Supinaam Demerara, presented by Mr. F. V. McConnell to the British Museum. Annals and Magazine of Natural History, (8) 6: 184-189.

1911. The mammals of the tenth edition of Linnaeus; an attempt to fix the types of the genera and the exact bases and localities of the species. Proceedings of the Zoological Society of London, 1911: 120157.

- 1923. New subspecies of Metachirus. Annals and Magazine of Natural History, (9) 11: 602-607.

- 1927a. The Godman-Thomas expedition to Peru-V. On mammals collected by Mr. R. W. Hendee in the Province of San Martin, N. Peru; mostly at Yurac Yacu. Annals and Magazine of Natural History, (9) 19: 361-375.

- 1927b. The Godman-Thomas expedition to Peru-VI. On mammals from the upper Huallaga and 
neighboring highlands. Annals and Magazine of Natural History, (9) 20: 594-608.

1928a. The Godman-Thomas expedition to Peru-VII. The mammals of the Río Ucayali. Annals and Magazine of Natural History, (10) 2: 249-265.

- 1928b. The Godman-Thomas expedition to Peru-VIII. On mammals obtained by Mr. Hendee at Pebas and lquitos, upper Amazonas. Annals and Magazine of Natural History, (10) 2: 285-294.

Thrasher, J. D., M. Barenfus, S. T. Rich, and D. V. SCHUPE. 1971. The colony management of Marmosa mitis, the pouchless opossum. Laboratory Animal Science, 21: 526-536.

Tiedemann, D. F. 1808. Erster Band allgemeine Zoologie, Mensch und Säugthiere. Landshut, 1808.

Tipton, V. J., R. M. Altman, and C. M. KeEnan. 1966. Mites of the subfamily Laelaptinae in Panama (Acarina: Laelaptidae), pp. 23-45, pls. 1-36. In Wenzel, R. L., and V. J. Tipton, eds.. Ectoparasites of Panama. Field Museum of Natural History, Chicago.

Tipton, V., AND C. MAchado. 1972. Fleas of Venezuela. Brigham Young University Scientific Bulletin, Biological Series, 17(6): $1-115$.

TIPTON, V. J., AND E. MÉndeZ. 1966. The fleas (Siphonaptera) of Panama, pp. 289-338, pls. 47-93. In Wenzel, R. L., and V. J. Tipton, eds., Ectoparasites of Panama. Field Museum of Natural History, Chicago.

Trapp, E., and R. E. Shope. 1967. U.S. Public Health Service Publication, 1760: 249-252. (ex Potkay, S. 1977. Diseases of Marsupials). In Hunsaker, D., II, ed., The Biology of Marsupials. Academic Press, New York.

TRIBE, C. J. 1990. Dental age classes in Marmosa incana and other didelphoids. Journal of Mammalogy, 71: 566-569.

TSCHUDt, J. J. voN. 1844. Untersuchungen über die Fauna Peruana. Thierologie. Scheitlin und Zollikofer, St. Gallen.

TUtTlE, M. D., L. K. TAFT, AND M. J. Ryan. 1981. Acoustical location of calling frogs by Philander opossums. Biotropica, 13(3): 233-234.

TYNDAle-Biscoe, C. H. 1973. Life of Marsupials. Elsevier, New York.

. 1980. Observations on the biology of marsupials in Colombia and Venezuela. Research Reports of the National Geographic Society, 12: 711-720.

Tyndale-Biscoe, C. H., And M. Renfree. 1987. Re- productive Physiology of Marsupials. Cambridge University Press, Cambridge, xiv + 476 pp.

Tyson, E. 1698. Carigueya seu Marsiupiale Americanum or the anatomy of an opossum. Philosophical Transactions of the Royal Society of London, 5: 105, 1576.

VIEIRA, C. A. DE C. 1945. Sôbre uma coleccão de mammíferos de Mato Grosso. Arquivos Zoologia, São Paulo, 4(10): 395-429.

— 1949. Xenartros, marsupiais do Estado de Sāo Paulo. Arquivos Zoologia, São Paulo, 7: 325-362.

WAGNER, J. A., ED., 1843. Die Säugthiere in Abbildungen nach der Natur mit Beschreibungen von Dr. Johann Christian Daniel Schreber. Supplementband 3. Erlangen.

- 1855. Die Säugthiere in Abbildungen nach der Natur mit Beschreibungen von Dr. Johann Christian Daniel Schreber. Supplementband 5. Leipzig, pp. xxvi +810 .

Waterhouse, G. R. 1841. The Naturalist's Library, Conducted by Sir William Jardine, Bart. Mammalia, Vol. XI, Marsupialia or Pouched Animals. Edinburgh.

- 1846. A Natural History of the Mammalia. 1, Marsupialia, or Pouched Animals. Hippolyte Bailliére, London, $553 \mathrm{pp}$.

WERNECK, F. L. 1949. Host associations of the lice of mammals. In HoPKINs, G. H. E. Proceedings of the Zoological Society of London, I19(1949-1950): 387607.

Wied-Neuwied, Maximilian, Prinzen zu. 1826. Beiträge zur Naturgeschichte von Brasilien. Weimar, 2: 1620.

WILSON, D. E. 1970. Opossum predation: Didelphis on Philander. Journal of Mammalogy, 51(2): 386-387.

WINGE, H. 1893. Jordfundne og nulevende Pungdyr (Marsupialia) fra Lagoa Santa, Minas Gerais, Brasilien. E. Museum Lundii, 2(II): I-149.

Wood, F. D., AND S. F. Wood. 1941. American Journal of Tropical Medicine, 21: 335.

Yates, T. L., and J. W. Froenlich. 1984. Noteworthy records of mammals from eastern Honduras. The Southwestern Naturalist, 29(4): 5! I-514.

YunkER, C. E., and F. J. Radovsky. 1966. The dermanyssid mites of Panama (Acarina: Dermanyssidae), pp. 83-I03. In Wenzel, R. L., and V. J. Tipton, eds., Ectoparasites of Panama. Field Museum of Natural History, Chicago. 



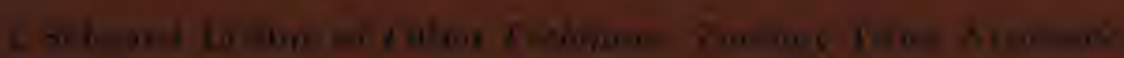

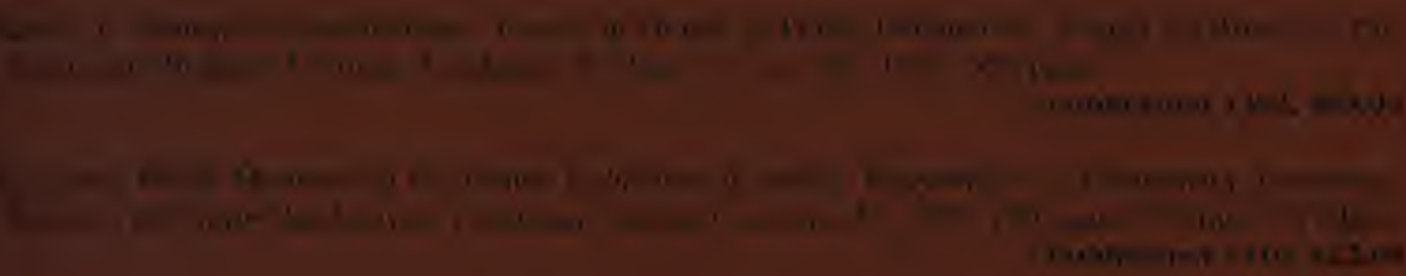

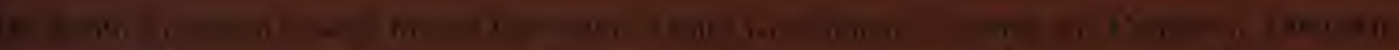

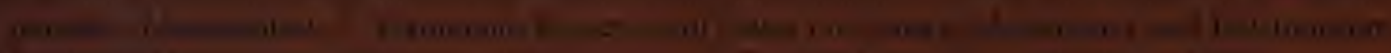

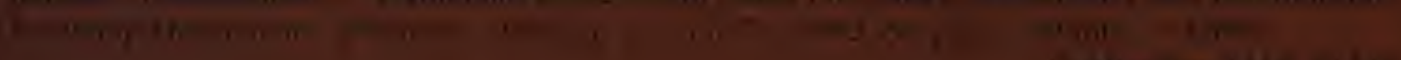

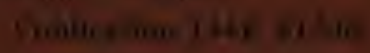

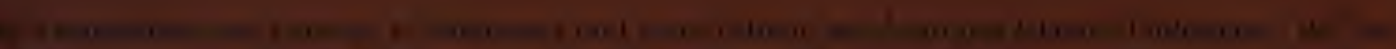

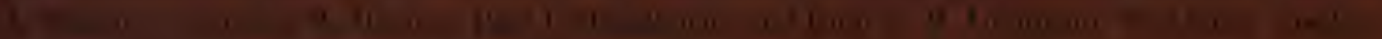

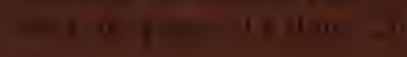

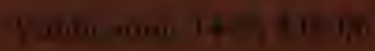

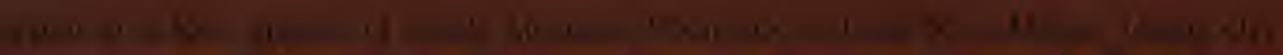

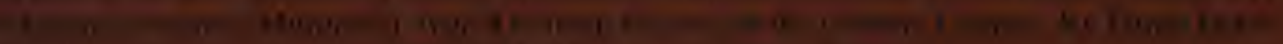

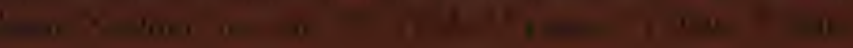

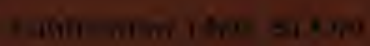

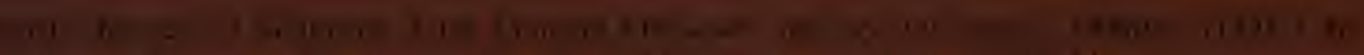

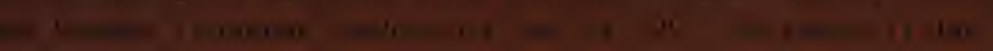

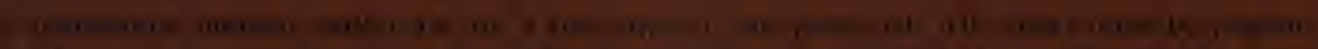

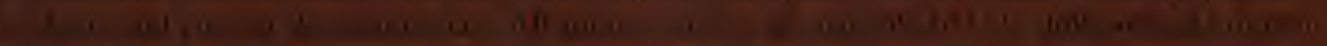

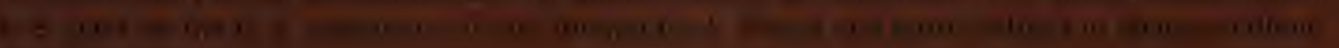
1 $19=1$

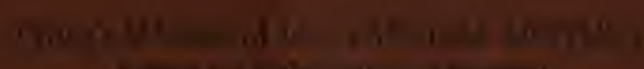

$$
\begin{aligned}
& \text { a) } 1 \text { a } 1
\end{aligned}
$$

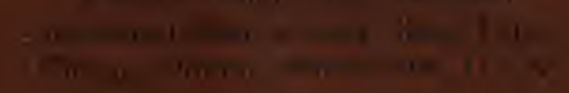




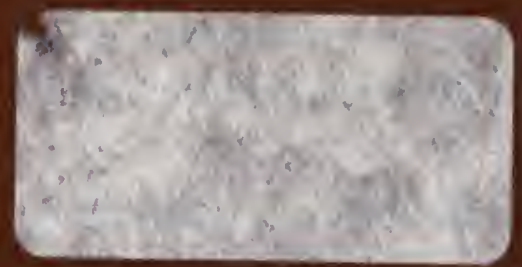




